\title{
Small nucleolar RNAs in chondorgenic differentiation and osteoarthritis
}

Citation for published version (APA):

Meekels-Steinbusch, M. M. F. (2020). Small nucleolar RNAs in chondorgenic differentiation and osteoarthritis. [Doctoral Thesis, Maastricht University]. ProefschriftMaken.

https://doi.org/10.26481/dis.20200924mm

Document status and date:

Published: 01/01/2020

DOI:

10.26481/dis.20200924mm

Document Version:

Publisher's PDF, also known as Version of record

\section{Please check the document version of this publication:}

- A submitted manuscript is the version of the article upon submission and before peer-review. There can be important differences between the submitted version and the official published version of record.

People interested in the research are advised to contact the author for the final version of the publication, or visit the DOI to the publisher's website.

- The final author version and the galley proof are versions of the publication after peer review.

- The final published version features the final layout of the paper including the volume, issue and page numbers.

Link to publication

\footnotetext{
General rights rights.

- You may freely distribute the URL identifying the publication in the public portal. please follow below link for the End User Agreement:

www.umlib.nl/taverne-license

Take down policy

If you believe that this document breaches copyright please contact us at:

repository@maastrichtuniversity.nl

providing details and we will investigate your claim.
}

Copyright and moral rights for the publications made accessible in the public portal are retained by the authors and/or other copyright owners and it is a condition of accessing publications that users recognise and abide by the legal requirements associated with these

- Users may download and print one copy of any publication from the public portal for the purpose of private study or research.

- You may not further distribute the material or use it for any profit-making activity or commercial gain

If the publication is distributed under the terms of Article $25 \mathrm{fa}$ of the Dutch Copyright Act, indicated by the "Taverne" license above, 


\title{
Small nucleolar RNAs in chondrogenic differentiation and osteoarthritis
}

\author{
Mandy Maria Franciscus Meekels-Steinbusch
}


Author: Mandy Meekels-Steinbusch

Copyright: @Mandy Meekels-Steinbusch, 2020. All rights reserved

Cover image copyright and artwork: @Professional Visual Artist René Teresa Campbell, The Fabric of Life

Lay-out: Mandy Meekels-Steinbusch, Jeroen Meekels, Dennis Hendriks - DeskTop Publisher ProefschriftMaken

Publisher: Global Academic Press

Printed by: ProefschriftMaken

First edition

ISBN/EAN: 978-94-6380-903-0

NUR code: 923 Cell and Molecular Biology

Financial support for the publication of the thesis

Netherlands society for Biomaterials and Tissue Engineering (NBTE)

Maastricht University / CAPHRI

\section{Research funding}

Netherlands Organization for Scientific Research (NWO), Deutsche Forschungsgemeinschaft (DFG), Dutch Arthritis Association (Reumafonds), Nederlands Orthopedisch Research en Educatie Fonds (Anna Fonds) 


\title{
Small nucleolar RNAs in chondrogenic differentiation and osteoarthritis
}

\author{
Proefschrift \\ Ter verkrijging van de graad van doctor aan de Universiteit Maastricht, \\ op gezag van de Rector Magnificus, Prof.dr. Rianne M. Letschert \\ volgens het besluit van het College van Decanen, \\ in het openbaar te verdedigen \\ op donderdag 24 september 2020 om 12.00 uur \\ door \\ Mandy Maria Franciscus Meekels-Steinbusch
}




\section{Promotoren}

Prof. Dr. L.W. van Rhijn

Prof. Dr. T.J.M. Welting

\section{Beoordelingscommissie}

Prof. Dr. J.F.C. Glatz (voorzitter)

Prof. Dr. H.G. Brunner

Dr. B. Cillero-Pastor

Dr. A.Y. Nossent (Leids Universitair Medisch Centrum)

Prof. Dr. G.J.V.M. van Osch (Erasmus MC Rotterdam) 


\section{Voor baby Meekels-Steinbusch}

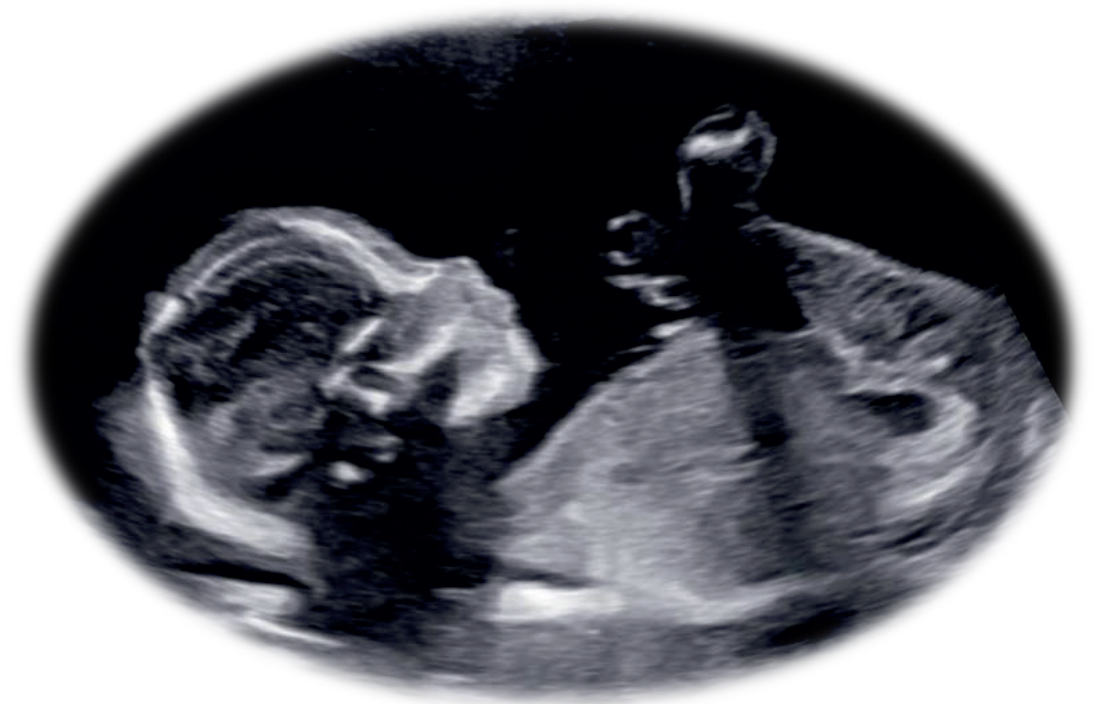





\section{Content}

$\begin{array}{lll}\text { Chapter } 1 \text { General introduction } & 11\end{array}$

Chapter 2 Expression of RMRP RNA is regulated in chondrocyte hypertrophy

61 and determines chondrogenic differentiation - Nature Scientific Reports: Sci Rep 7 (2017), 6440, doi: 10.1038/s41598-017-06809-5

Chapter 3 The antiviral protein viperin regulates chondrogenic differentiation 99 via CXCL10 protein secretion - Journal of Biological Chemistry: J Biol Chem (2019) 294 (13) 5121-5136, doi: 10.1074/jbc.RA119.007356

Chapter 4 Adaptation of the protein translational apparatus during ATDC5 chondrogenic differentiation - Thesis chapter, manuscript in preparation

Chapter 5 Serum snoRNAs as biomarkers for joint ageing and post traumatic 167 osteoarthritis - Nature Scientific Reports: Sci Rep 7 (2017), 43558, doi: $10.1038 /$ srep43558

Chapter 6 General discussion

Chapter 7 An overview of proposed molecular interactions from this thesis 221

Chapter 8 Valorization

Chapter 9 Summary

Curriculum Vitae

List of publications

List of presentations

List of honors and achievements 



\section{Chapter 1 \\ General introduction}


Chapter 1 


\section{General introduction}

Cartilage is an important structural component of the body; essential during walking, sports and everyday life functioning. It is a firm tissue, but it is softer and much more flexible than bone. It is a connective tissue found in many areas of the body including the articulating joints between the bones (e.g. the elbows, knees, hips and ankles), the end of the ribs, between the vertebrae in the spine, in the ears and nose and in the bronchial tubes and trachea ${ }^{1}$. It is essential for functions such as breathing, hearing, articulation and locomotion. The cartilage present in diarthrotic joints is hyaline articular cartilage and is present in a 2-4 $\mathrm{mm}$ layer on the articulating ends of the long bones. Its function is to provide a smooth, lubricated surface for articulation that is able to withstand the enormous amount of intensive and repetitive forces that are associated with locomotion ${ }^{2-4}$, combined with low friction. Cartilage tissue is populated by specialized cells called chondrocytes. The chondrocytes produce large amounts of extracellular matrix (ECM) which, amongst others, is composed of collagen fibers and proteoglycans. There are no blood vessels in cartilage to supply the chondrocytes with nutrients. Instead, nutrients, oxygen etc. diffuse from the synovial cavity and surrounding tissues through the cartilage to reach the chondrocytes. Due to the lack of vascularization, cartilage is largely hampered in reparative capacity, as compared to other tissues ${ }^{1}$. This has major implications for cartilage diseases such as osteoarthritis (OA), the most prevalent degenerative joint disease that is characterized by articular cartilage destruction ${ }^{5}$. 


\subsection{Chondrogenic differentiation and osteoarthritis}

\subsubsection{Chondrogenic differentiation in the context of skeletal development}

Longitudinal bone growth during skeletal development and lengthening of the limbs depends on endochondral ossification. Endochondral ossification is a multistage process in the growth plates of developing long bones. Chondrogenic differentiation is part of the endochondral ossification process and it encompasses the commitment and differentiation of chondro-progenitor cells towards chondrocytes ${ }^{6}$. In vivo, during skeletal development, chondrogenic differentiation is initiated from local mesenchymal progenitor cells that reside in the growth plate's resting zone ${ }^{6}$. In addition to playing an essential role in endochondral ossification, chondrogenic differentiation also provides articulating joint surfaces with functional cartilage during development, maintains cartilage integrity and is involved in bone fracture healing, 6, Growth plates are populated with highly proliferative chondrocytes, which differentiate into mineralizing hypertrophic chondrocytes and subsequently die from apoptosis or transdifferentiate into osteoblasts ${ }^{8-10}$. The remaining mineralized ECM provides a scaffold for osteoblasts and osteoclasts to adhere and remodel, setting the stage for bone deposition and thus longitudinal bone growth and limb development ${ }^{11,12}$ (Figure 1). In the proliferative zone of the growth plate, Sox9 drives the expression of type II collagen (Col2a1) ${ }^{13}$ and Aggrecan (Acan) ${ }^{14}$. These two proteins are major markers for this stage of chondrogenic differentiation. Transcription factors Runx2 and Mef2c drive the expression of type $X$ collagen (Col10a1) ${ }^{15,16}$ during the hypertrophic phase of chondrogenic differentiation. Together with alkaline phosphatase $(\mathrm{Alpl})^{17}$, these proteins are major markers for the hypertrophic phase of chondrogenic differentiation. 

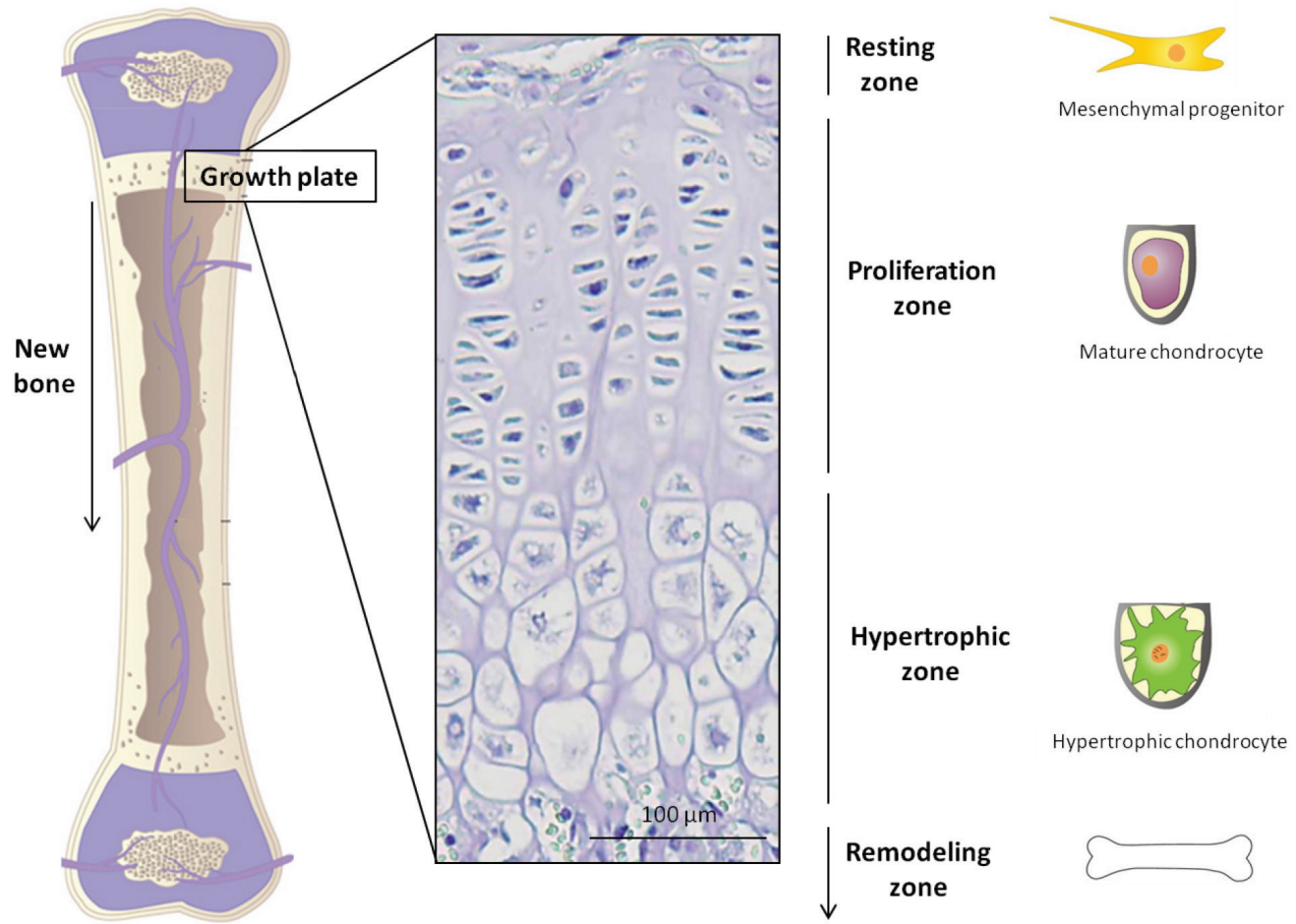

Figure 1. Chondrogenic differentiation; the process in the growth plate through which long bones form. Here, chondrocytes (= cartilage cells) remodel to bone. In the growth plate we can distinguish: resting zone chondrocytes (mesenchymal progenitor cells), proliferative chondrocytes (i.e. mature chondrocytes that appear in a structured train-like formation), hypertrophic chondrocytes and a remodeling zone. The highly proliferative chondrocytes from the proliferation zone differentiate into mineralizing hypertrophic chondrocytes that either die from apoptosis or transdifferentiate into osteoblast ${ }^{8-10}$ in the remodeling zone. The remaining ECM in the remodeling zone provides a scaffold for osteoblasts and osteoclasts to adhere and remodel, setting the stage for bone apposition and thus longitudinal bone growth and limb development ${ }^{11,12}$. The bone cartoon (left) was adapted from Wolpert, L. ${ }^{18}$. The H\&E stained histological microphotograph is from an 8 weeks-old mouse growth plate. 


\subsubsection{Mediators of chondrogenic differentiation during skeletal development}

Sizes and shapes of bones need to be carefully coordinated to allow efficient movement. Therefore, both autocrine and paracrine regulators, as well as hormones in the bloodstream, control both skeletal development and bone remodeling throughout life $\mathrm{e}^{12}$. Thus, chondrogenic differentiation, as part of the bone growing process, can be influenced by a number of growth factors and chondrogenic mediators. Bone morphogenetic proteins (BMPs), WNTs, transforming growth factors (TGFs), fibroblast growth factors (FGFs), hedgehog proteins, insulin-like growth factors (IGFs) and retinoids are essential for chondrogenic differentiation during growth plate development and bony skeletal element formation. These locally produced factors are joined by systemic factors such as growth hormone, thyroid hormone, estrogen, androgen, vitamin D and glucocorticoids to control skeletal development ${ }^{12,19}$.

\section{Hedgehog and PTHrP signaling}

Indian hedgehog $(\mathrm{IHH})$ is a master regulator of bone development, coordinating growth plate chondrocyte proliferation, chondrocyte differentiation and osteoblast differentiation ${ }^{20}$. $\mathrm{IHH}$ is a member of the hedgehog family of secreted ligands and is closely related to Sonic Hedgehog (SHH). SHH is the main ligand that activates Patched-1-mediated GLI-signaling, which is crucial in limb bud patterning during embryogenesis ${ }^{20}$. The relevance of SHH-mediated signaling is highlighted by a mouse model where targeted disruption of SHH leads to a holoprosencephaly (forebrain of the embryo fails to develop into two hemispheres) phenotype and abnormal development of the spine, ribs and distal limbs ${ }^{21}$. IHH is synthesized by growth plate chondrocytes leaving the proliferative pool (i.e. the prehypertrophic chondrocytes) and by early hypertrophic chondrocytes; and it controls hypertrophic differentiation ${ }^{12}$. PTHrP, on the other hand, keeps chondrocytes in a proliferative state and counteracts hypertrophic differentiation ${ }^{12,22}$. Disruption of the PTHrP gene in mice causes skeletal dysplasia with accelerated chondrocyte maturation ${ }^{23}$, and a constitutively active mutant PTH/PTHrP receptor has been found in Jansen-type human 
metaphyseal chondrodysplasia, a disease characterized by delayed skeletal maturation ${ }^{24}$. Furthermore, homozygous mutations in $\mathrm{IHH}$ cause acrocapitofemoral dysplasia; an autosomal recessive disorder with cone-shaped epiphyses in the hand and hips ${ }^{25}$. Together, the balance between IHH and PTHrP is an important determinant regulating chondrogenic differentiation (Figure 2).

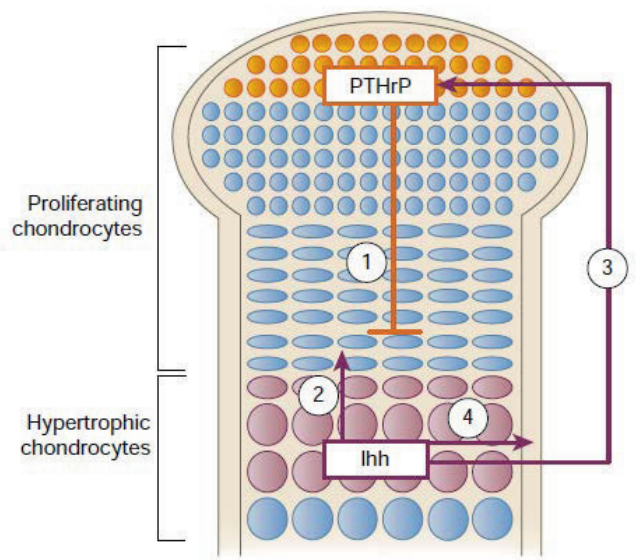

Figure 2. IHH/PTHrP negative feedback loop of chondrogenic differentiation. PTHrP is secreted from perichondrial cells and chondrocytes at the end of long bones. 1) In the proliferative zone, PTHrP acts on the receptors of proliferating chondrocytes to keep them in a proliferative state and thereby, delay the production of $\mathrm{IHH}$. 2) When the source of PTHrP production is sufficiently distant, IHH is produced. IHH acts on its receptor Patched-1 to increase hypertrophic differentiation. 3) IHH has been shown to again stimulate new production of PTHrP at the end of long bones. 4) IHH also acts to convert the cells of the hypertrophic zone into osteoblasts, for the purpose of remodeling. Text adapted from Kronenberg et al. $^{12}$, figure from Kronenberg et al. ${ }^{12}$ : copyright clearance received

\section{FGFs}

FGF ligands are polypeptide growth factors that regulate several developmental processes including cellular proliferation, differentiation, migration, morphogenesis and patterning ${ }^{26}$. FGF signaling itself constitutes a large and complex form of molecular regulation and hence 
FGFs and their receptors serve many functions in the developing organism. FGF-FGFR signaling is critical to the developing axial and craniofacial skeleton. Recent studies have shown that FGF signaling crucially regulates chondrocyte proliferation and differentiation. For example, FGFs have been shown to stimulate SOX9 expression ${ }^{12}$ and it has been demonstrated that basic FGF (bFGF; and also known as FGF2) is a negative regulator of chondrocyte hypertrophic maturation ${ }^{27}$. Humans express 18 different known FGF ligands and four FGF receptors (FGFR) 28 . FGF signaling generally follows one of three transduction pathways: RAS/MAP kinase (controls cell proliferation and differentiation ${ }^{29}$ ), PI3/AKT (regulates cell survival and fate determination ${ }^{30}$ ) or PLCY (influences cell morphology, migration and adhesion $\left.{ }^{30}\right)^{31}$. Each pathway likely regulates specific cellular behaviors. Aberrant expression of FGFs and improper activation of FGFRs are associated with various pathological conditions, unregulated growth and tumorigenesis ${ }^{32}$. In addition, aberrant FGF signaling has been implicated in many skeletal abnormalities including achondroplasia ${ }^{33}$ (over-activation of FGFR3, caused by a gain-of-function mutation, which impairs chondrocytes within the growth plate and results in decreased inhibition of endochondral ossification ${ }^{34}$ ) and craniosynostosis (premature fusion of the cranial sutures) ${ }^{35}$. Molecular cross-talk between the BMP and the FGF signaling pathways has also been described (BMPsignaling infers with FGF-induced ERK phosphorylation) ${ }^{36,37}$.

\section{BMPs, GDFs, TGF- $\beta$ s and SMADs}

Bone morphogenetic proteins (BMPs), growth and differentiation factors (GDFs) and transforming growth factors (TGFs) have multiple crucial roles in chondrogenic differentiation during skeletal development. BMPs, GDFs and TGF- $\beta$ s are members of the TGF- $\beta$ superfamily of paracrine factors that activate heterodimeric receptors with serine/threonine kinase activity ${ }^{38}$ with major implications for the differentiated cell fate. For example, hypertrophic differentiation during chondrogenic differentiation of progenitor cells is stimulated by BMP-2 but suppressed by BMP-739, GDF-5 is a BMP family member described to induce both chondrogenic differentiation and hypertrophy ${ }^{40}$ and TGF- $\beta$ isoforms are well known to induce chondrogenesis and are imperative for cartilage homeostasis via activation of SMAD2/3 signaling ${ }^{41-44}$. In most cases, TGF- $\beta$ superfamily 
signaling is initiated by ligand-induced dimerization of serine/threonine receptor kinases and phosphorylation of the cytoplasmic signaling molecules SMAD2 and SMAD3 for the TGF$\beta /$ activin pathway, or SMAD1/5/8 for the BMP and GDF ${ }^{45}$ pathway. Carboxy-terminal phosphorylation of SMADs by activated receptors results in their partnering with SMAD4 (a Co-SMAD) and translocation to the nucleus to facilitate target gene regulation. Activated SMADs regulate a diversity of cell biological events by partnering with transcription factors (such as Runx2 to induce BMP2-induced osteogenic signaling ${ }^{46}$ ) resulting in cell-state specific modulation of transcription ${ }^{42}$. SMAD6 and SMAD7 are known as inhibitory SMADs ${ }^{43}$. The importance of TGF- $\beta$ superfamily signaling for skeletal development and homeostasis is further emphasized by conditions in which components of the TGF- $\beta$ superfamily signaling pathway are mutated. A review by Wang et al. indicated that knock-out of major players in BMP-signaling, including BMPs, SMADs and BMP receptors, show severe skeletal phenotypes ${ }^{47}$. In addition, multiple monogenic diseases of the skeleton caused by mutations of TGF- $\beta$ and TGF- $\beta$-related genes have been identified as well, such as diaphyseal dysplasia Camurati-Engelmann (TGF- $\beta 1$ ), Marfan syndrome (TGF- $\beta 2$ ), Brachydactyly type A2 (BMP-2), Grebe dysplasia (GDF5), Loeys-Dietz syndrome type C (aortic aneurysms and dissections with early onset OA; SMAD3) and Myhre syndrome (SMAD4) ${ }^{48,49}$. In addition, Osteogenesis Imperfecta (brittle bone disease) can also be caused by BMP-1 loss of function ${ }^{50,51}$.

\section{Canonical $\beta$-catenin and non-canonical $\mathrm{Ca}^{2+}$ WNT signaling}

WNTs comprise a family of highly conserved soluble mediators that are responsible for a large number of important developmental and homeostatic processes ${ }^{52-54}$. WNT proteins generally activate one of two main signaling pathways: the canonical WNT/ $\beta$-catenin pathway or the non-canonical WNT/Ca ${ }^{2+}$ pathway. The defining event in canonical WNT signaling is the cytoplasmic accumulation of $\beta$-catenin and its subsequent nuclear translocation and activity. The canonical WNT/ $\beta$-catenin pathway is activated by binding of one of the canonical WNT ligands to a Frizzled family receptor and an low density lipoporotein receptor-related protein (LRP) 5/6 co-receptor, which passes the signal via

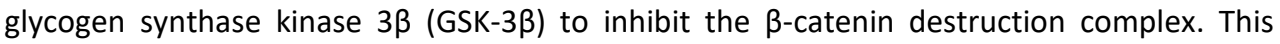
causes accumulation of $\beta$-catenin in the cytoplasm and eventually its translocation into the 
nucleus ${ }^{55,56}$. This enables transcriptional coactivation with T-cell factor/lymphoid enhancing factor (TCF/ LEF) transcription factors which are active in the transcription of WNT-target genes, such as RUNX2 $2^{56}$. Once in the nucleus $\beta$-catenin can act as a transcriptional activator or repressor of specific genes, depending on the co-factors present, highlighting the complexity of WNT signaling and nuclear $\beta$-catenin activity ${ }^{52}$. The canonical WNT/ $\beta$-catenin pathway has been well-established to be associated with chondrocyte differentiation and hypertrophy ${ }^{55,57-60}$. In the non-canonical WNT signaling pathway many intercellular signaling molecules are involved, including inositol triphosphate (IP3)-intercellular calcium, MAPKs and G-protein RhoA/Rho-associated kinase (Rock). The receptors/co-receptors that mediate this type of WNT signaling include Frizzled, receptor tyrosin kinase (Ryk) and tyrosineprotein kinase transmembrane receptor $(\mathrm{Ror} 2)^{61}$. The non-canonical WNT/Ca ${ }^{2+}$ pathway leads to release of intracellular $\mathrm{Ca}^{2+}$. Elevated $\mathrm{Ca}^{2+}$ can activate phosphatase calcineurin, which leads to dephosphorylation of NFAT transcription factors, their accumulation in the nucleus and downstream non-canonical WNT target gene expression.

To highlight a few findings; WNT-3A and WNT-5A are two prominent ligands of the Wnt signaling pathway that are crucial in chondrocyte differentiation ${ }^{62}$. In fact, WNT signaling was first linked to skeletal development in 1994, when WNT3A-deficient embryos were found to exhibit axial defects ${ }^{63}$. Later, in humans it was shown that a homozygous WNT-3 loss-of-function mutation causes defects in limb formation and craniofacial and urogenital development ${ }^{64}$. In addition, mutations in the WNT1 gene have been linked to Osteogenesis Imperfecta $65-68$, a disease characterized by brittle bones which are easily fractured. More broadly assessed, results from a multitude of studies reveal that $\beta$-catenin-dependent canonical and $\beta$-catenin-independent non-canonical WNT signaling pathways have multiple roles in regulation of cartilage development, growth and maintenance ${ }^{61,69-84}$. For example, canonical $\beta$-catenin-dependent WNT signaling is required for progression of endochondral ossification and growth of axial and appendicular skeletal elements, while excessive activation of this signaling can cause severe inhibition of initial cartilage formation and growth plate organization and function in mice ${ }^{61}$. Mouse embryos that express a constitutive active form of $\beta$-catenin in developing cartilage under the control of the type 2 collagen promoter/enhancer exhibit severe dwarfism and skeletal deformities. Cartilaginous elements in this mouse present with a strongly reduced synthesis of cartilage matrix 
molecules and fail to organize growth plate structure ${ }^{73-75}$. On the contrary, inactivation of $\beta$ catenin signaling also induces severe skeletal deformity in mice. Conditional ablation of $\beta$ catenin in the skeletal cells of the limb strongly impairs long bone formation with inhibition of chondrocyte hypertrophy and osteoblast differentiation ${ }^{80-82}$. In addition, ablation of $\beta$ catenin also revealed disturbed or delayed development of the growth plate hypertrophic zone and calcification, associated with altered bone formation ${ }^{81,83}$. The results from these studies provide clear evidence that the WNT/ $\beta$-catenin pathway is an essential mediator to control cartilage and bone development. On the other hand, non-canonical WNT signaling has also been shown to be important in columnar organization of growth plate chondrocytes ${ }^{78}$. In addition, WNT5a has been shown to play a critical role in embryonic development ${ }^{85}$. WNT5a binds to the extracellular cysteine-rich domain of ROR2 (receptor tyrosine kinase-like orphan receptor 2$)^{86}$. A close functional relationship between ROR2 and WNT5a is consistent with the fact that mice deficient for either gene develop skeletal phenotypes including dwarfism, facial abnormalities and shortened limbs and tails ${ }^{87}$. There is also evidence that WNT signaling regulates and is required for cartilage development and growth in human. Robinow syndrome (autosomal dominant (MIM180700) and autosomal recessive (MIM268310)) is a family of rare skeletal dysplasia syndromes associated with WNT5A and ROR2 mutations, respectively, and it is characterized by dysmorphic features resembling a fetal face, mesomelic limb shortening, hypoplastic external genitalia in males, and renal and vertebral anomalies ${ }^{88}$. ROR2 mutations are also linked to Brachydactyly, Type B1 (MIM113000), which presents itself by malformation of the distal phalanges ${ }^{89}$. These genetic features provide evidence that non-canonical WNT-mediated pathway is also important in the formation and morphogenesis of cartilage skeletal elements in human.

\section{IGF}

Lastly, IGF signaling is essential for the regulation of tissue formation and remodeling, bone growth, prenatal growth, brain development and muscle metabolism. Cellular effects of IGF signaling are mediated through the IGF-1 receptor; a transmembrane tyrosine kinase that phosphorylates intracellular substrates, resulting in the activation of Pi3K/AKT and RAS/RAF/MEK/ERK intracellular signaling cascades $^{90}$. The insulin-like growth factors 
including IGF-1, IGF-2 and insulin, are single-chain polypeptides that share a similar secondary structure, with three $\alpha$-helixes and three disulphide bonds ${ }^{91}$. Despite significant structural similarity, each ligand can result in unique signaling outcomes. For example, IGF-2 is unable to compensate for the loss of IGF-1 activity in patients with IGF-1 deficiency, leading to severe growth and mental retardation ${ }^{92-94}$. The main endocrine action of IGF-1 is to mediate the growth-promoting effects of pituitary growth hormone (GH), however, IGF1-mediated paracrine/autocrine effects are also essential in the modulation of cellular growth, proliferation, differentiation and survival against apoptosis. The GH/IGF-1-axis provides the main stimulus for bone growth regulation by activating the osteoblast differentiation program, stimulating chondrocyte proliferation in the growth plate and modulating tubular reabsorption of phosphate and 25-hydroxyvitamin D3-1 $\alpha$-hydroxylase activity in the kidney ${ }^{95,96}$. The fundamental role of IGF-1 in regulating bone formation is demonstrated by analysis of IGF-1-deficient mice, which exhibit skeletal malformations, delayed mineralization, reduced chondrocyte proliferation and increased chondrocyte apoptosis $^{97}$. Furthermore, dysregulation of IGF-1 signaling due to impairment in the postreceptor signaling machinery contributes to multiple diseases in human including osteoporosis and reduced fetal growth in utero ${ }^{98}$.

\section{Mediators of chondrogenic differentiation; a summary}

Summarizing the effects of the above mentioned mediators of skeletal development, we can conclude that chondrogenic differentiation can be broadly influenced by a multitude of growth factors and other chondrogenic mediators that regulate pathways, any of which could be affected during disease.

\subsubsection{Osteoarthritis}

Osteoarthritis (OA), the most prevalent degenerative joint disease, is an age-related musculoskeletal disease and a common cause of chronic disability worldwide ${ }^{5}$. During 
ageing there is a general age-dependent reduction of functional capacity and stress resistance, which is associated with an increased risk of morbidity and mortality. The articular joint and its articular cartilage is particularly affected by ageing ${ }^{99}$. However, OA is not simply a process of wear and tear, but rather an abnormal remodeling of joint tissues, driven by katabolic mediators within the affected joint. The most important risk factors for OA include age, sex, prior joint injury, obesity, diabetes, genetic predisposition and mechanical factors, including malalignment and abnormal joint shape ${ }^{100,101}$. Despite the multi-factorial nature of $\mathrm{OA}$, the macroscopic pathological changes observed in osteoarthritic joints have common features that affect the entire joint structure, resulting in pain, deformity and loss of joint function ${ }^{102}$. Figure 3 provides a schematic representation of a healthy joint versus a joint suffering from severe OA. The pathological changes seen in OA joints include degradation of the articular cartilage, thickening of the subchondral bone, formation of osteophytes, variable degrees of synovitis, degeneration of ligaments and, in the knee: the menisci, and hypertrophy of the joint capsule ${ }^{102}$. Changes in the periarticular muscles, nerves, bursa, and local fat pads (e.g. Hoffa) that may contribute to OA or its symptoms can also be observed ${ }^{102}$. The pathological changes observed in all of the different joint tissues imply that OA can be considered as a disease of the joint as an "organ", that will result in total failure of the joint ${ }^{102}$.
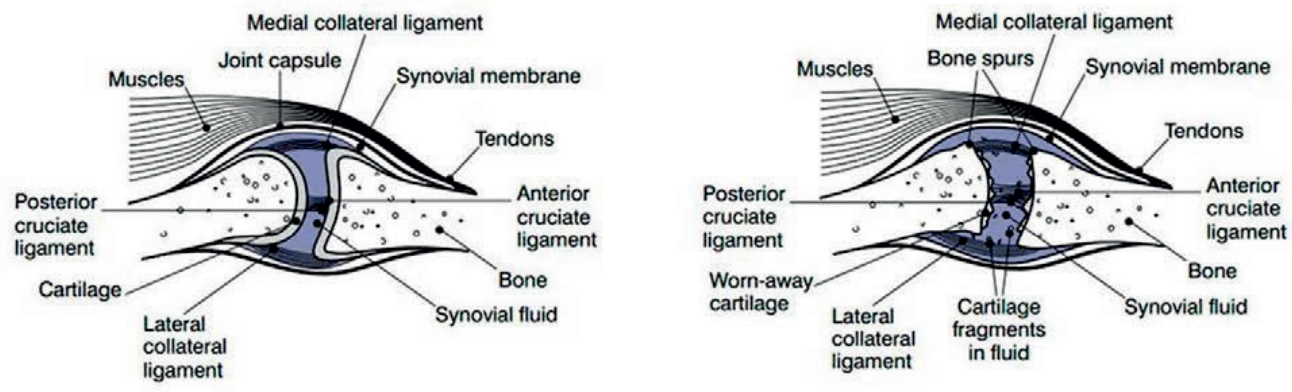

Figure 3. Schematic representation of a healthy joint (left) and a joint with severe

osteoarthritis. In a healthy joint, the ends of the bones are encased in smooth cartilage. Together, they are protected by a joint capsule lined with a synovial membrane that produces synovial fluid. The capsule and fluid protect the cartilage, muscles and connective tissues. In osteoarthritis, the cartilage is being eroded and bone ends may experience friction. Spurs (osteophytes) can grow from 
the edge of the bones. Cartilage fragments may be present in the synovial fluid. The joint will often feel stiff and painful. Picture from Medicine $\mathrm{Net}^{103}$ C2016, WebMD, LLC. All rights reserved

\section{The pathogenesis of OA}

The pathogenesis of $\mathrm{OA}$ is complex and not yet well understood, but it involves the interaction of multiple factors, ranging from genetic predisposition to mechanical, molecular and environmental components. One of the main hallmarks of OA is articular cartilage destruction due to an imbalance between the synthesis and degradation of the cartilage ECM components and the earliest degenerative changes in cartilage appear at the joint surface in areas where mechanical forces, and in particular shear stress, are greatest ${ }^{104}$. In adult cartilage, in a healthy homeostatic, non-stressed state, chondrocytes are relatively quiescent and there is little turnover of the cartilage matrix. In $O A$, articular chondrocytes are "activated", which is characterized by cell proliferation, cell cluster formation and increased production of matrix proteins and matrix-degrading enzymes ${ }^{105}$. Matrix-degrading enzymes in $\mathrm{OA}$ include aggrecanases and collagenases (i.e. members of the matrix metalloproteinases (MMPs)), as well as several cysteine proteinases ${ }^{106}$. In early OA, matrix degradation may be due to MMP-3 and ADAMTS-5, which degrade aggrecan, followed by increased activity of collagenases (e.g. MMP-13 is highly active in degrading type II collagen) ${ }^{102}$. The inflammatory cytokines IL-1 $\beta$, TNF- $\alpha, \mathrm{IL}-17$ and IL-18 act to increase synthesis of MMPs, decrease levels of MMP enzyme inhibitors and attenuate ECM synthesis ${ }^{105}$. It has been suggested that, cell-biologically, this is an injury-like response, leading to cartilage matrix remodeling, inappropriate hypertrophy-like chondrocyte differentiation and cartilage calcification ${ }^{105}$. Articular chondrocytes must express appropriate genes to maintain cartilage tissue homeostasis. However, the gene expression alterations observed in OA cartilage indicate a replay of chondrogenic differentiation as observed during growth plate development, with the expression of a genetic program associated with chondrocyte hypertrophy, leading to endochondral ossification and articular cartilage destruction ${ }^{107}$. 


\section{Differential chondrogenic mediator signaling in $\mathrm{OA}$}

\section{WNT and OA}

In this respect, WNT signaling has been implicated in OA. Transgenic mouse models show that both decreased and increased levels of $\beta$-catenin result in cartilage damage ${ }^{108,109}$. Overexpression of $\beta$-catenin led to high expression of hypertrophy markers (i.e. MMP-13, type $X$ collagen) by articular chondrocytes, while conditional knock-out of $\beta$-catenin led to chondrocyte apoptosis ${ }^{108,109}$. Both models show cartilage destruction, but the model showing elevated $\beta$-catenin levels and elevated hypertrophy markers demonstrated a pattern of joint destruction more similar to spontaneous $\mathrm{OA}^{110}$. Elevated canonical WNT signaling drives OA development by increasing RUNX2 activity and thereby boosting hypertrophic differentiation of chondrocytes ${ }^{111-113}$. In addition, WNT/ $\beta$-catenin signaling has been shown to regulate initiation of chondrocyte hypertrophy by inhibiting PTHrP signaling activity $^{112}$. It has been shown that TGF- $\beta$ and BMP signaling is modulated by WNT signaling, most probably by altering SMAD signaling ${ }^{114-117}$. Moreover, Sox9 and $\beta$-catenin interact in their control of chondrocyte differentiation. Sox9 and $\beta$-catenin reciprocally influence other each other's degradation and compete in combination with TCF-LEF for promoter binding sites associated with chondrocyte differentiation ${ }^{75,118}$. Recent genetic data linked a polymorphism in the FrzB gene, encoding for a WNT-binding protein, to the development of $\mathrm{OA}$, suggesting that abnormal WNT signaling also contributes to $\mathrm{OA}^{119}$. Blom et al. found that $\beta$-catenin expression, along with other WNT-Frz-related genes, was upregulated in cartilage and synovium during experimental OA in mice. The authors identified WISP-1 (capable of inducing cartilage-degrading enzymes such as MMPs, ADAMTS-4 and ADAMTS$5)$, independently of the catabolic cytokine IL-1B, as a crucial WNT-signaling mediator ${ }^{120}$. Moreover, recent studies using a transgenic OA mouse model with conditional activation of the $\beta$-catenin gene in articular chondrocytes showed that upregulation of $\beta$-catenin signaling is most probably responsible for the conversion of normal articular chondrocytes into OA-like chondrocytes: chondrocyte maturation genes were activated along with the induction of matrix degradation ${ }^{108,121}$. 


\section{ALK, SMAD, TGF- $\beta$ signaling and OA}

A striking age-related change in articular cartilage is the loss of ALK-5 and SMAD2/3 phosphorylation ${ }^{122}$. Phosphorylated SMAD3 is a known blocker of chondrocyte hypertrophy and it is thus believed that ageing-related loss of protective SMAD phosphorylation is a driver of chondrocyte hypertrophy in $O A$ cartilage ${ }^{110}$. Furthermore it has been generally accepted nowadays that uncontrolled synthesis and signaling of TGF- $\beta$ superfamily members by several joint tissue compartments greatly contributes to OA development and progression ${ }^{123,124}$. HtrA1, a broad-substrate enzyme involved in degradation of the pericellular matrix and expressed in early experimental OA and hypertrophic chondrocytes, is a potent blocker of TGF- $\beta$ signaling ${ }^{125-128}$. In addition, it has been shown that during late stage $\mathrm{OA}$, chondrocytes that are involved in the repair of cartilage damage, show high levels of SMAD2/3 and SMAD1/5/8 phosphorylation ${ }^{110}$, indicating TGF- $\beta$ superfamily-mediated activation of these cells during OA. Although BMP-2 has potent anabolic actions, BMP activity in chondrosarcoma cells and in murine cartilage was shown to induce OA-like changes by stimulation of MMP-13 ${ }^{123}$. Lack of TGF- $\beta$ signaling results in OA-like changes with terminal differentiation of chondrocytes. As shown in genetic mouse models, TGF- $\beta$ mediates this effect by binding to the ALK5 type-I TGF- $\beta$ receptor and subsequent activation of the SMAD2/3 intracellular signaling route ${ }^{129,130}$.

\section{Indian Hedgehog and OA}

Hedgehog signaling was also described to play a role in $\mathrm{OA}$. IHH levels are higher in $\mathrm{OA}$ synovial fluid compared to non-AO synovial fluid and exposure of chondrocytes to $\mathrm{IHH}$ induces the expression of type $X$ collagen $^{131}$. Lin et al. described increased expression of hedgehog targets in human $\mathrm{OA}$ samples and mouse articular cartilage after surgical OA induction. Amplified IHH target gene expression correlated with advanced disease stages and hedgehog proteins were described to stimulate the expression of the aggrecanase ADAMTS-5 via the transcription factor Run $\times 2^{132}$. Causal evidence for a central role of $\mathrm{IHH}$ in OA cartilage pathology comes from a study where cartilage-specific genetic deletion of IHH was performed. Genetic IHH deletion protects against OA development, strongly indicating that $\mathrm{IHH}$ is a critical determinant in OA-related cartilage pathology ${ }^{133}$. 


\section{GH, IGF-1 and OA}

With respect to the GH/IGF-1 axis, it was shown in a rat model for OA that chronic GH deficiency caused an increased severity of OA articular cartilage lesions ${ }^{134}$. Anabolic IGF-1 signaling is antagonized by increased levels of IGF-binding proteins, which then negatively regulate IGF-1 signaling in chondrocytes during $\mathrm{OA}^{135}$. In contrast, patients with $\mathrm{GH}$ deficiency had significantly less incidence of OA than patients of a control population ${ }^{136}$. This shows that $\mathrm{GH}$ is a risk factor in the development of $\mathrm{OA}$, but up until now its role remains a controversy.

\section{FGFs and OA}

FGF23 provokes chondrocyte hypertrophy ${ }^{137}$, and in line with a role in OA it has been shown that OA chondrocytes express FGF23 to higher levels than healthy chondrocytes. On the contrary, FGFR3 is important for inhibition of chondrocyte hypertrophy. In concert with a positive role of FGFR3 in chondrocyte homeostasis ${ }^{138}$, bFGF/FGF-2, displays beneficial effects on articular cartilage homeostasis as FGF-2-deficient mice present with increased OA development with age as compared to wild-type mice. This is due to an increased expression of ADAMTS-5 aggrecanase ${ }^{139}$, resulting in cartilage loss.

Finally, retinoids exert multiple effects relevant to the OA disease process. For example, components of the retinoid signaling pathways were shown to be upregulated in the tibiofemoral joints of patients with $\mathrm{OA}$, and all-trans-retinoic acid treatment of human cartilage explants led to significant increase in MMP-13 and aggrecanases expression, enzymes that are both involved in the key proteolytic processes implicated in $\mathrm{OA}^{140}$.

\section{Chondrogenic mediators and OA; a summary}

Taken together, many locally acting mediators playing a crucial role in the regulation of chondrocyte proliferation and differentiation during endochondral ossification are also involved in OA pathogenesis and cartilage disease progression ${ }^{141}$. Defining markers of joint ageing and early OA may enable a prediction of the risk of onset of OA, enabling early 


\section{Chapter 1}

intervention. In addition, $\mathrm{OA}$ is characterized by a non-symptomatic, pre-radiographical phase that if identified would allow earlier diagnosis (radiographic changes are only evident later in disease progression) and treatment, before irreversible cartilage damage has set-it.

\section{2 snoRNAs}

\subsection{1 snoRNAs and their function}

Small nucleolar (sno)RNAs are a group of non-coding (i.e. untranslated) RNA molecules of variable length ( \pm 60 to $200 \mathrm{nt}^{142,143}$ ) mostly required for posttranscriptional ribosomal RNA (rRNA) maturation ${ }^{144}$. snoRNAs reside in the nucleolus of the cell, which is the most prominent substructure within the nucleus of a cell (Figure 4), and the site of ribosome assembly, rRNA transcription and RNA processing ${ }^{145}$.
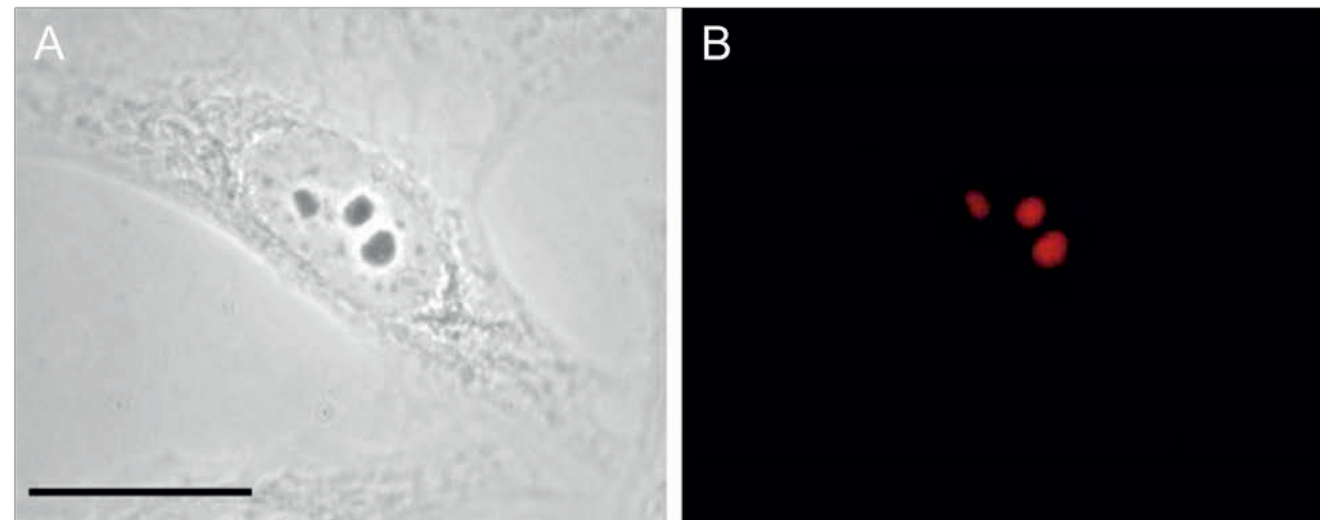

Figure 4. Visualization of the nucleoli of a cell. A) HEp-2 carcinoma cell with nucleus and three nucleoli. B) Nucleolar staining of RPP20 nucleolar protein (red color) 


\section{Transcription, translation and ribosomes}

Cells require large numbers of ribosomes to meet the demand for protein synthesis during proliferation, development, differentiation, homeostasis and disease ${ }^{146,147}$. This is supported by the observation that actively proliferating mammalian cells contain approximately 5 to 10 million ribosomes that must be replenished and synthesized each time the cell divides ${ }^{145}$. All proteins are synthesized via ribosomes in the process of transcription and translation (Figure $\mathbf{5 A})^{148}$. The nucleolus is a ribosome production factory, designed to fulfill the need for large-scale production of rRNAs and assembly of the ribosomal subunits ${ }^{145}$. Mammalian ribosomes contain four species of RNA, designated 5S, 5.8S, $18 \mathrm{~S}$ and 28S rRNA and a myriad of ribosomal proteins (Figure 5B) ${ }^{149,150}$.

A

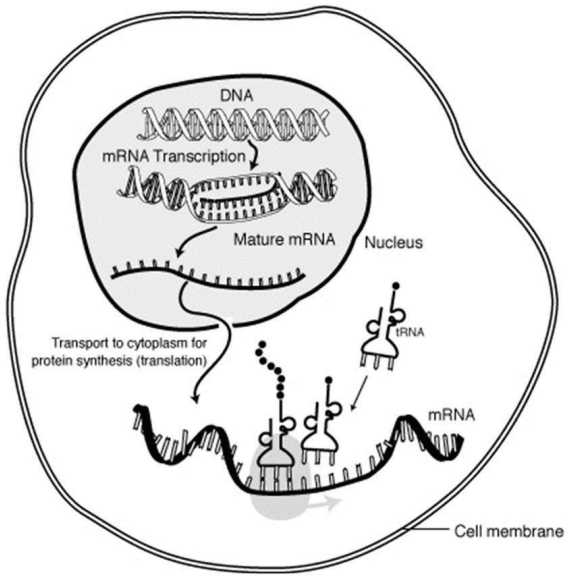

B

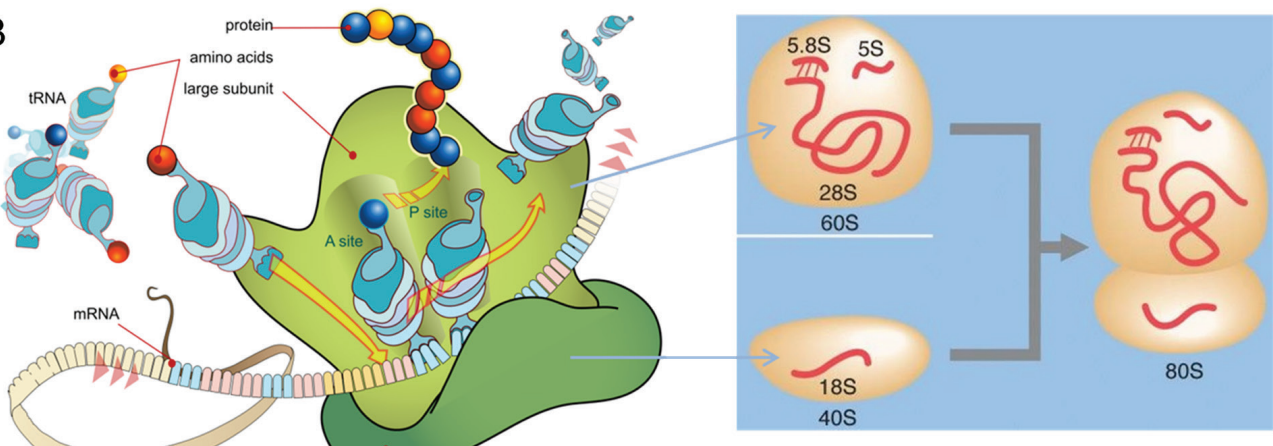


Figure 5. Transcription and translation; and the eukaryotic $80 \mathrm{~S}$ ribosome. A) mRNA carries genetic information from the DNA to the ribosome. The ribosome decodes the information from the mRNA and as a result produces a specific amino acid chain also known as a polypeptide. The polypeptide then folds into an active protein and performs its functions in the cell. Decoding of information by the ribosome is facilitated by inducing the binding of complementary tRNA anticodon sequences to the mRNA codons (three-base codes). The tRNAs carry the specific amino acids corresponding to the mRNA codons to the ribosome ${ }^{151}$. Figure from the Talking glossary of genetic terms: illustration freely available and may be used without special permission ${ }^{152}$. B) Two subunits can be distinguished within the ribosome: a $40 \mathrm{~S}$ small ribosomal subunit, consisting of the $18 \mathrm{~S}$ rRNA and approximately 33 ribosomal proteins ${ }^{150}$, and a $60 \mathrm{~S}$ large ribosomal subunit, consisting of the $5 \mathrm{~S}$, $5.8 \mathrm{~S}$ and $28 \mathrm{~S}$ rRNAs and approximately 46 ribosomal proteins ${ }^{149,150}$. Figure adapted from Cellular and Molecular Biology ${ }^{153,154}$, the work has been released in the public domain, the author ${ }^{154}$ grants anyone the right to use this work for any purpose, without any conditions.

\section{rRNA processing}

Mammalian 5.8S, $18 \mathrm{~S}$ and $28 \mathrm{~S}$ rRNAs are transcribed as a single polycistronic unit within the nucleolus by RNA polymerase I, yielding a $47 \mathrm{~S}$ ribosomal precursor RNA ${ }^{150}$. To meet the demand for transcription of large numbers of rRNA molecules, cells contain multiple copies of the $47 \mathrm{~S}$ rRNA gene. The human genome contains approximately 200 copies of the $47 \mathrm{~S}$ gene and approximately 2000 copies of the 5S rRNA gene ${ }^{145}$. The importance of ribosome production is particularly evident in oocytes, in which the rRNA genes are amplified to support the synthesis of large numbers of ribosomes required for early embryonic development ${ }^{145,148}$. The transcribed 475 pre-rRNA is cleaved at both ends to generate the $45 S$ pre-rRNA ${ }^{155}$ and then, requiring a number of snoRNA-dependent actions, processed by two complex alternative pathways to produce the mature $18 \mathrm{~S}$ rRNA for the $40 \mathrm{~S}$ small ribosomal subunit and the mature $5.8 \mathrm{~S}$ and $28 \mathrm{~S}$ rRNAs of the $60 \mathrm{~S}$ large ribosomal subunit ${ }^{156}$ (Figure 6). Transcription of the 5S rRNA (a 60S subunit component) takes place outside of the nucleolus and is catalyzed by RNA polymerase III $^{145,157}$. During ribosome assembly ribosomal proteins are imported to the nucleolus from the cytoplasm and begin to assemble on pre-rRNA prior to its cleavage ${ }^{145,150}$. As the pre-rRNA is processed, additional ribosomal proteins and $5 \mathrm{~S}$ rRNA assemble to form pre-ribosomal particles. The final steps of 
maturation follow the export of pre-ribosomal particles to the cytoplasm, yielding the $40 \mathrm{~S}$ and 605 ribosomal subunits ${ }^{145,150}$.

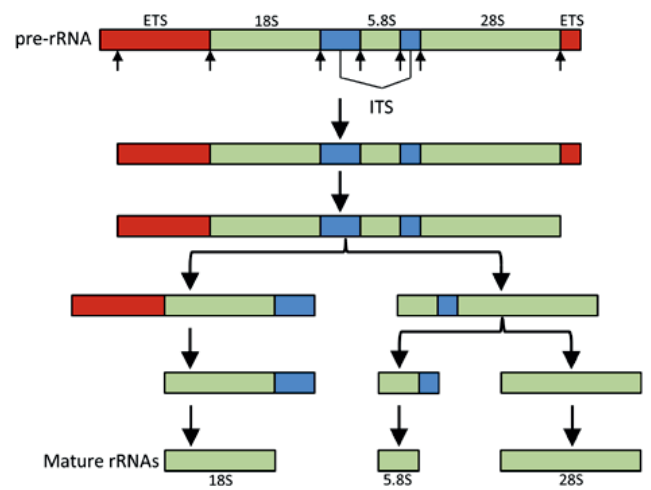

Figure 6. Processing of pre-rRNA. The

475 pre-rRNA transcript contains external transcribed spacers at both ends and internal transcribed spacers between the sequences of $18 \mathrm{~S}, 5.8 \mathrm{~S}$ and $28 \mathrm{~S}$ rRNAs. The pre-rRNA is processed via a complex series of cleavages. A simplified schematic overview is illustrated here for human $47 \mathrm{~S}$ pre-rRNA, to yield the mature rRNA species. Figure: own work, processing adapted as described by Henras ${ }^{150}$ : open access article under the terms of the Creative Commons Attribution Non-commercial License, which permits use, distribution and reproduction in any medium, provided the original work is properly cited and is not used for commercial purposes.

\section{Ribosome function}

Two important ribosome aspects that should be highlighted are the peptidyltransferase center (PTC) and the decoding center. The PTC can be considered the catalytic heart of the ribosome ${ }^{158}$. It resides in the large ribosomal subunit and catalyzes the two principal chemical reactions of protein synthesis: peptide bond formation and peptide release $\mathrm{e}^{158}$. The decoding center, which is present in the small subunit, binds both mRNA and tRNAs and mediates accurate codon-anticodon interactions and tRNA translocation ${ }^{159}$. The decoding center is composed of five specific helices in $18 \mathrm{~S}$ rRNA (i.e. helix 18, 24, 31, 34 and 44) $160,161$. Specific nucleotides involved in decoding have been revealed by mutational analysis, in which translation efficiency, fidelity, or tRNA translocation were affected ${ }^{159}$. These findings established the importance of the proper sequence and structure of the decoding center in controlling tRNA selection and translocation, and ensuring efficient, accurate translation ${ }^{162-}$ 164 . 


\section{snoRNAs and their relationship with rRNA}

The maturation of ribosomes involves various small nucleolar ribonucleoprotein (snoRNP) particles that are required at specific stages ${ }^{150}$. SnoRNPs consist of individual snoRNAs complexed with eight to ten proteins ${ }^{145}$. The snoRNPS belong to two families characterized by specific evolutionary conserved motifs in their RNA component, the so called C/D or H/ACA boxes ${ }^{165,166}$. Certain snoRNPs, including snR30/U17 and U14 function as RNA chaperones assisting early cleavages of the pre-rRNA into 18S, 5.8S and 28S rRNA. In addition, the most abundant nucleolar snoRNA, U3, which is present in about 200,000 copies per cell ${ }^{145}$, is required for the initial cleavage of pre-rRNA within the $5^{\prime}$ external transcribed spacer sequences ${ }^{167}$. U8 snoRNA is responsible for cleavage of pre-rRNA to $5.8 \mathrm{~S}$ and $28 \mathrm{~S}$ rRNAs, RMRP snoRNA matures the 5 ' end of $5.8 \mathrm{~S}$ rRNA by cleaving pre-rRNA in the internal transcribed spacer $1^{168,169}$, and U22 snoRNA is responsible for cleavage of pre-rRNA to $18 \mathrm{~S}$ rRNA $^{145,170}$.

However, most box C/D and box H/ACA snoRNPs catalyze 2'O-ribose methylation and uridine isomerization into pseudouridine (respectively) at specific positions of the rRNA species ${ }^{150,171-174}$. In mammalian cells pre-RNA processing involves the 2'O-ribose methylation of approximately a hundred ribose residues. In addition, fully maturated rRNA species contain about a hundred pseudouridines. Together these post-transcriptional modifications are being carried out by approximately 200 individual snoRNAs ${ }^{145,150}$. Most of these modifications occur during or shortly after synthesis of the pre-rRNA and only a small number take place at later stages of pre-rRNA processing ${ }^{145}$. Each snoRNA base-pairs with pre-rRNA at the vicinity of the nucleotide to be modified (target RNA sequence complementarity), thereby guiding the enzymatic modification carried out by one of the core proteins of the snoRNPs: the methyltransferase Nop1/fibrillarin in the box C/D snoRNP and the pseudouridylase Cbf5p/Nap57p/Dyskerin in the H/ACA snoRNPs ${ }^{150}$ (Figure 7: box $C / D$ and $H / A C A$ snoRNAs). Furthermore, there is evidence that guide snoRNA targets are not limited to rRNA $^{175,176}$, but for example can also guide chemical modifications on snRNAs and mRNAs ${ }^{177}$. The modified rRNA nucleotides are mostly found in functionally important regions of the ribosome, such as the PTC and the decoding center, and are necessary for efficient and accurate translation ${ }^{159,166}$. 

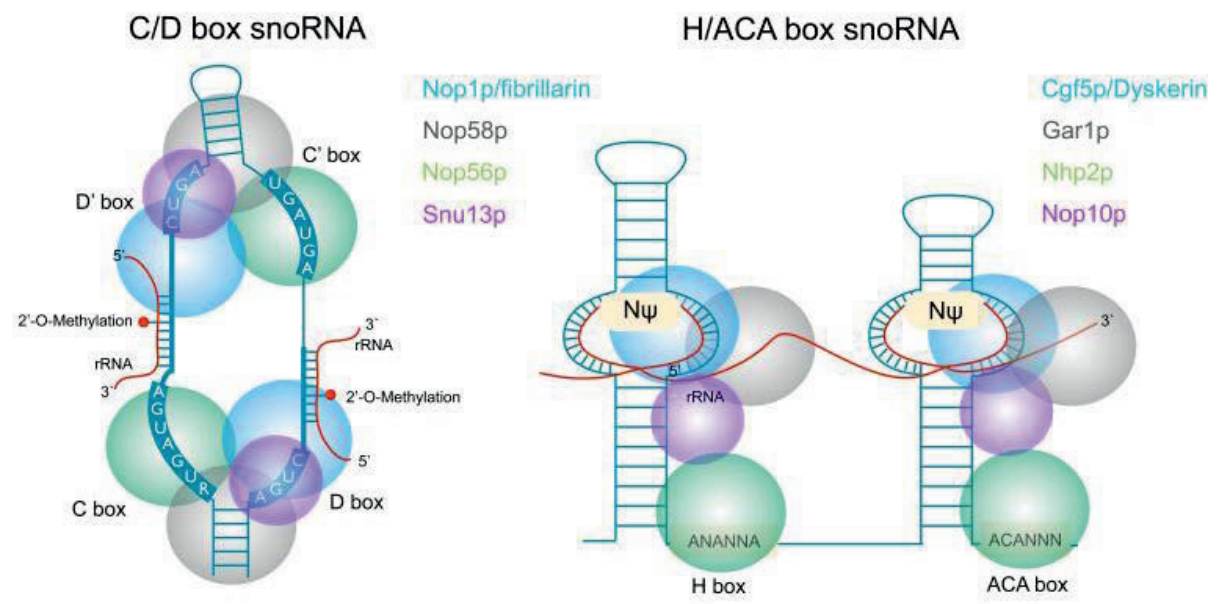

Figure 7. snoRNA classes. The boxed sequences C (UGAUGA) and D (CUGA) are hallmarks of the C/D box snoRNAs while the boxed sequences $H$ (for: hinge region, and where $\mathrm{N}$ stands for any nucleotide) and the trinucleotide ACA are hallmarks of the H/ACA box snoRNAs. These conserved boxed sequences are important for the association with the protein components that are required to form the functional snoRNP complexes. C/D box snoRNAs associate with several proteins, including fibrillarin, which is the methyltransferase that is involved in the 2'-O methylation of particular ribonucleotides. The H/ACA box snoRNAs associate with proteins such as the pseudouridine synthase Cgf5p/dyskerin. Antisense sequences within the C/D and H/ACA box snoRNAs guide the snoRNP complex to the appropriate nucleotide within the target RNA (most often ribosomal RNA). The eukaryotic H/ACA box snoRNAs contain two hairpin domains with complementary regions flanking the uridine to be converted in the target rRNA. Figure adapted from Garofalo et al. ${ }^{178}$, (C) 2013 Di Leva and Garofalo. Licensee IntechOpen, this chapter is distributed under the terms of the Creative Commons Attribution 3.0 License, which permits unrestricted use, distribution, and reproduction in any medium, provided the original work is properly cited.

Although normally present in the nucleolus, it has been demonstrated that snoRNAs can be detected in serum ${ }^{179-182}$ as protein complexes ${ }^{180}$ or contained within extracellular vesicles in serum $^{179}$. It is however not clear whether disease-associated RNAs detected in the circulation result from local tissue disturbances and cell death, or whether they are actively secreted via exosomes or microvesicles ${ }^{183-185}$. 
Relevant for the musculoskeletal field, changes in the level of snoRNAs circulating in body fluids were shown to be induced by trauma. Box C/D snoRNAs SNORD48 and SNORD38 were found to be significantly higher in serum of patients with anterior cruciate ligament (ACL) injury ${ }^{180}$. These snoRNAs could therefore be evaluated as possible biomarkers for early diagnostics of osteoarthritis following joint injury.

\subsection{2 snoRNAs in cell function and disease}

The different snoRNA functions described above may be implicated in the initiation and progression of pathology and the study of snoRNA function is expected to have significant diagnostic and therapeutic value. Changes in rRNA maturation and modification caused by changed snoRNA levels can cause disruption of vital processes and, amongst others, can lead to diseases and transformation of normal cells into tumor cells ${ }^{186-188}$. Hence, studies aimed at evaluating snoRNA expression levels and function in mammalian cells is expected to deliver novel insights in snoRNA function in development and disease and underpin the development of diagnostic approaches and the potential generation of novel therapeutic agents.

Classically, snoRNAs have been investigated in yeast model systems and, although still quite scarce, the majority of the work in mammalian systems comes from the oncology field. In mammals the majority of snoRNAs are encoded within the introns of protein-coding on noncoding genes, so called 'host-genes' 189,190 . Notably, many host-genes are genes encoding ribosomal proteins. Therefore, an alteration of snoRNA expression may result from the oncogenic processes accompanied by changes in transcriptional activity of the host-gene, aberrations of nuclear maturation of the donor transcript or alterations in snoRNA turnover. In such a case, cellular levels of the specific snoRNA may not play a key role in the pathology, but the alteration itself may indicate tumor progression and reflect metastatic potential ${ }^{191}$. On the other hand, snoRNAs, the expression of which can be positively or negatively correlated with tumor cell proliferation and invasion, may have considerable impact on carcinogenesis and a number of snoRNAs could be classified as oncogenes when their expression goes far beyond the 'normal level'192. Specific increase in the expression level of several box C/D snoRNAs was detected in murine and human breast cancer cells ${ }^{193}$. 
The change in snoRNA expression levels was accompanied by an increase in fibrillarin expression, the core component of the box C/D snoRNP. High levels of fibrillarin were observed not only in breast cancer cells, but also in prostate and many other human cancers. Targeted repression of fibrillarin and other box C/D snoRNP proteins Nop56 and Nop58 led to a decrease in box C/D snoRNA levels and, reduced oncogenicity and enhanced induction of tumor suppressor p53 protein ${ }^{193}$. The box H/ACA snoRNA SNORA42, which is overexpressed in non-small cell lung carcinoma, was identified as a lung cancer oncogene ${ }^{192}$. Ribosomal RNA biogenesis is known to be more robust in cancer cells than in normal cells ${ }^{194}$. Therefore, it can be envisioned that the increase in snoRNA levels is necessary for the acceleration of rRNA maturation, ribosome assembly and protein synthesis during tumorigenesis. It should be noted that the relationship between the deregulation of snoRNA expression and rRNA biogenesis in diseases still remains elusive and thus further studies in the snoRNA research area have the potential to address this knowledge gap.

SnoRNA-guided modifications are not evenly distributed over the ribosomal RNAs. Instead they cluster in functionally important regions including the decoding and tRNA binding sites (the A-, P- and E-sites), the PTC and the intersubunit interface ${ }^{195-197}$. This distinctive spatial distribution, which has been conserved throughout evolution ${ }^{198,199}$ suggests that rRNA modifications play important roles in regulating ribosome function ${ }^{196}$. The importance of these clusters of modifications in translation fidelity has also been investigated and lack of subsets of modifications in the decoding center was shown to impair stop-codon termination and affect reading frame maintenance ${ }^{200}$. Alternations in the expression levels of snoRNAs, or their core enzymes fibrillarin and dyskerin, may directly alter translational regulation of major regulators of cell growth and differentiation ${ }^{194,201,202}$. As an example, deleting five H/ACA snoRNAs (snR10, snR34, snR37, snR42 and snR46) that guide six pseudouridylation modifications in the PTC in yeast ribosomes, resulted in defects in protein synthesis and growth ${ }^{203}$ and knockdown of fibrillarin or dyskerin impacts the mode of translation (Cap versus IRES-mediated translation) ${ }^{204,205}$. In addition, a study from HigaNakamine et al. ${ }^{206}$ showed that knockdown of box C/D snoRNAs SNORD26, SNORD44 or SNORD78 results in developmental defects in zebrafish. Knockdown of only one of these specific snoRNAs caused embryonic lethality, decreased body size, underdevelopment of the jaw, incomplete yolk sac extensions and hind brain defects. 
Mutations in dyskerin, the box H/ACA snoRNA pseudouridylase, and other telomerase components, are the cause of the condition Dyskeratosis Congenita ${ }^{207}$. Patients with Dyskeratosis Congenita are characterized by poor development of the teeth and nails. In addition, they often have impaired bone marrow function and are prone to develop pulmonary fibrosis. Patients may also have low bone mineral density 208,209 and increased risk for developing cancers ${ }^{210}$.

\subsubsection{RMRP snoRNA and Cartilage-hair hypoplasia}

The eukaryotic ribonuclease for mitochondrial RNA processing (RNase MRP) is a small nucleolar ribonucleoprotein particle mainly located in the nucleoli, consisting of the $R M R P$ snoRNA and several protein subunits (i.e. Rpp14, Rpp20, Rpp21, Rpp25, Rpp30, Rpp38, Rpp40, hPop1, hPop4 and hPop5) ${ }^{211}$ (Figure 8). Characterization of the RNase MRP particle showed that it contains an RNA species that is required for its enzymatic activity ${ }^{212}$. RNase MRP RNA (RMRP RNA) is nuclear encoded and classified as a snoRNA ${ }^{211}$. RNase MRP shares both functional and structural features with RNase $P^{211,213,214}$. RNase $P$ is involved in the processing of pre-tRNA. Like the RNase MRP particle, the RNase P particle contains an essential RNA subunit, also referred to as H1 RNA. Although the sequences of both RNA subunits are not highly conserved, they can be folded into similar secondary structures ${ }^{215,216}$. 


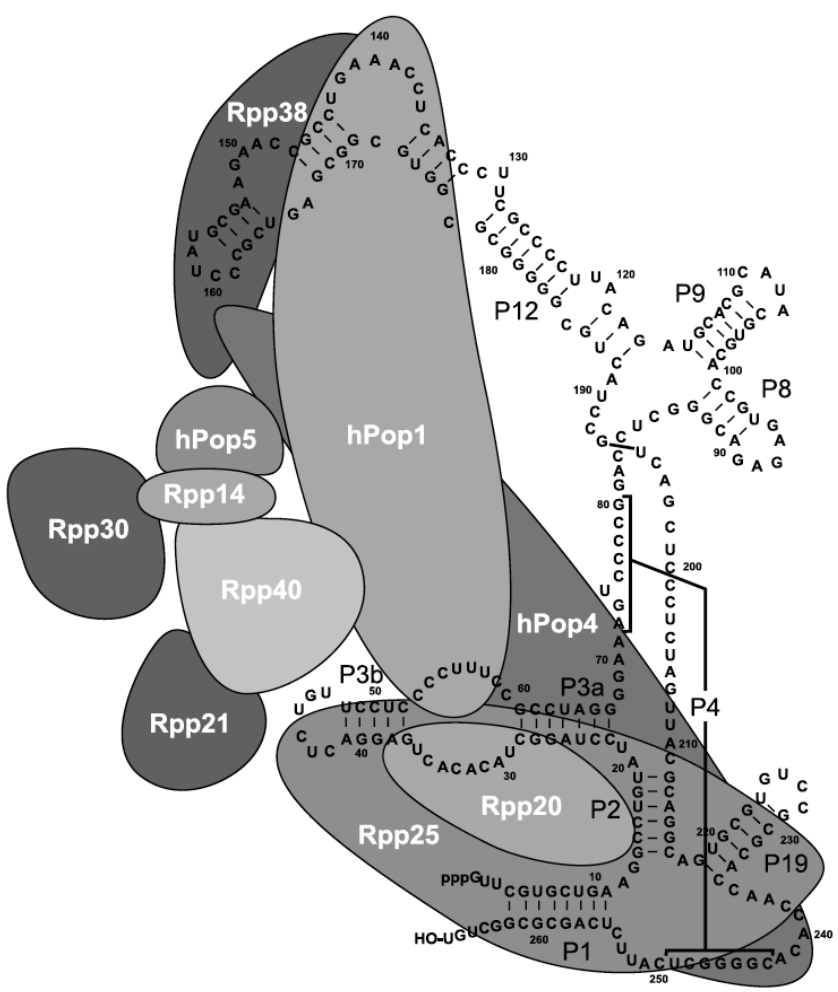

Figure 8. Model of the human RNase MRP complex. RMRP RNA associates with protein subunits Rpp14, Rpp20, Rpp21, Rpp25, Rpp30, Rpp38, Rpp40, hPop1, hPop4 and hPop5. Figure from Welting et al. ${ }^{211}$, reproduced with permission from Prof. Dr. Welting

RNase MRP belongs to a class of snoRNPs that does not 2'O-ribose methylate or pseudouridylate specific rRNA residues. Instead, RNase MRP acts as an endoribonuclease that cleaves several RNA substrates. The RMRP RNA can be classified as a ribozyme: RNA molecules that are capable of catalyzing specific biochemical reactions, similar to the action of protein-based enzymes ${ }^{217}$. RNase MRP has been reported to be involved in mitochondrial DNA replication ${ }^{218}$, cleaves pre-rRNA in the internal transcribed spacer 1 (ITS1) ${ }^{168,169}$ (Figure 9), plays a role in cell cycle regulation by cleaving cyclin b2 $\mathrm{mRNA}^{219}$, and is able to cleave viperin $\mathrm{mRNA}^{220}$. In addition, it has been shown that RMRP snoRNA and the telomeraseassociated reverse transcriptase (TERT) protein are able to form a complex that exerts RNAdependent RNA polymerase activity, which is able to generate a double stranded RMRP RNA molecule. Via a Dicer-dependent route siRNAs are generated from this molecule, down- 
regulating cellular $R M R P$ levels and providing a negative feedback mechanism controlling RMRP snoRNA levels 221 .

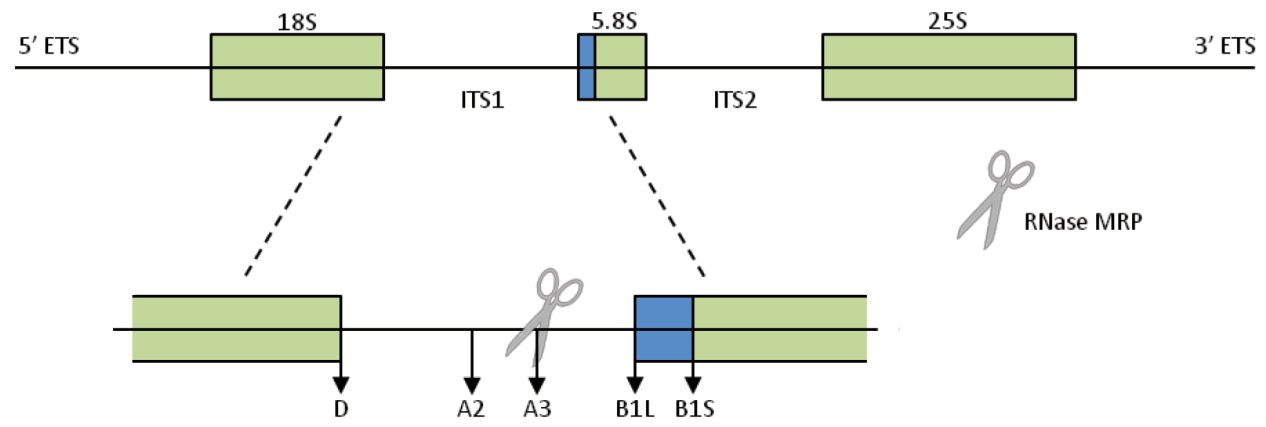

Figure 9. RNase MRP cleaves pre-rRNA in the internal transcribed spacer 1 (ITS1) at the A3 site. Hereby, RNase MRP matures the 5' end of 5.8S rRNA. Cleaving process adapted from Welting et al. ${ }^{222}$, with permission from the author

Mutations in the RMRP gene are the cause of a severe form of dwarfism known as the cartilage-hair hypoplasia ${ }^{223}$ (CHH: OMIM \#250250) - anauxetic dysplasia ${ }^{224}$ (AAD: OMIM \#607095) spectrum of disorders ${ }^{225}$ (Figure 10). $\mathrm{CHH}$ is also termed metaphyseal chondrodysplasia, McKusick-type ${ }^{226}$. To date, more than hundred individual $\mathrm{CHH}$-pathogenic mutations have been identified in the $R M R P$ gene $\mathrm{e}^{223}$. 


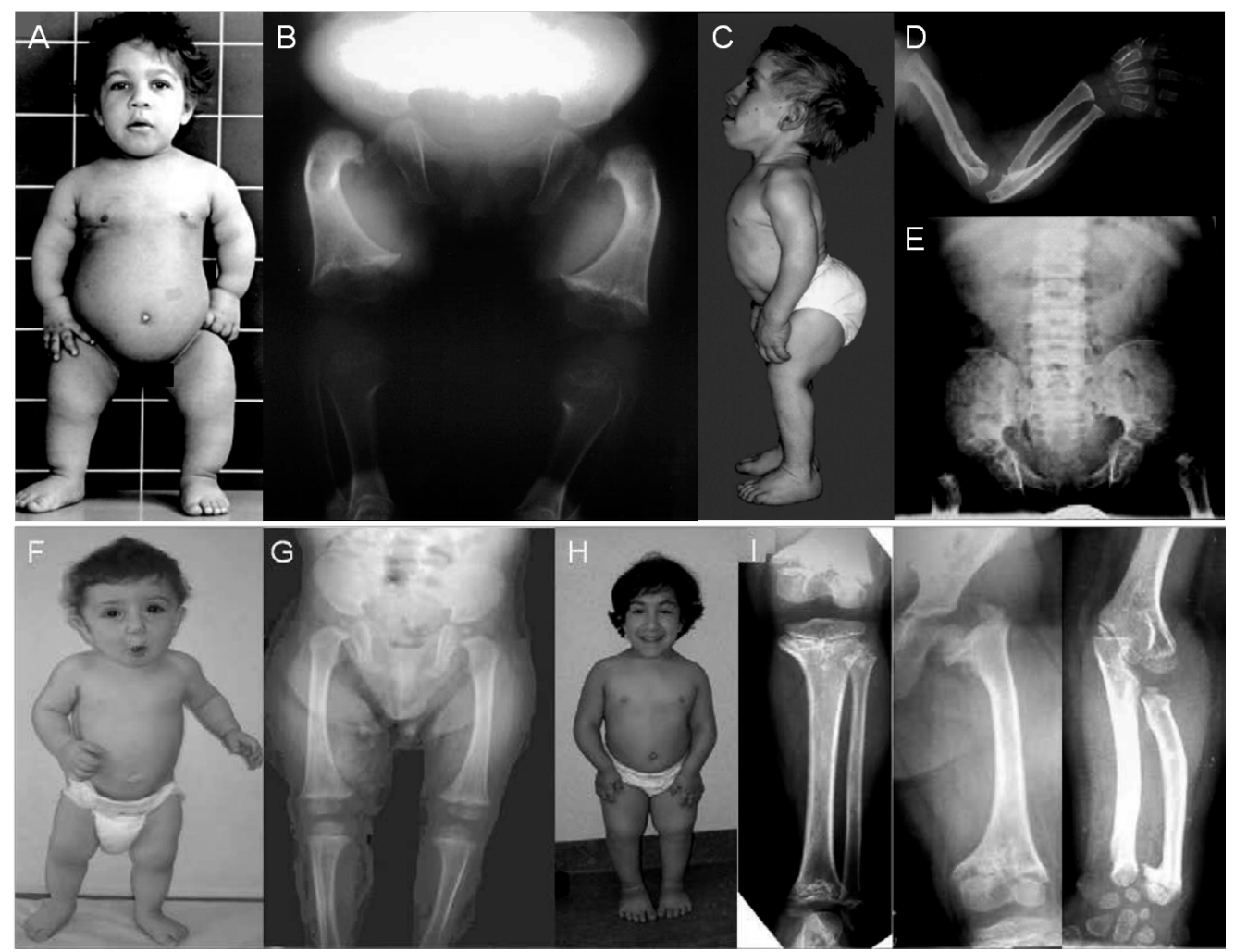

Figure 10. Phenotypic characterization of $\mathrm{CHH}$ patients. Mutations in the RMRP gene are the cause of a severe form of dwarfism known as $\mathrm{CHH}$. A) Clinical phenotype of a female patient at the age of four years with RMRP mutations $63 \mathrm{~T}>\mathrm{C}$ and duplication of $195 \mathrm{~T}^{227}$, copyright clearance received for use of Figure. B) Radiograph of the pelvis and femur of the same patient at 2 years. Note the short and severely bowed femur, under-ossification of the proximal tibia and increased acetabular angles ${ }^{227}$, copyright clearance received for use of Figure. C) Patient, aged 16 years, showing severely short stature (height: $74 \mathrm{~cm}$ ) with hyperlordosis (RMRP mutations: $14 \mathrm{G} \rightarrow \mathrm{A}$ and 90_91AG $\rightarrow G C)^{224}$, copyright clearance received for use of Figure. D) X-ray of the left arm of same patient at age 7, showing extremely retarded carpal ossification, as well as short, broad tubular bones $^{224}$, copyright clearance received for use of Figure. E) X-ray of the spine and pelvis at age 7 , showing decreased vertical dimension of the illia, unossified femoral necks and small, irregular capital femoral epiphyses ${ }^{224}$, copyright clearance received for use of Figure. F) 15 month old boy with triangular face and mildly disproportionate short-limbed dwarfism, with pudgy hands, diagnosed with compound heterozygous RMRP mutations -4_-23dupTACTCTGTGAAGCTGAGGAC and $146 G \rightarrow A^{228}$, Figure retrieved from the University of Zurich Open Repository and Archive. G) Lower 
extremity radiography of the boy from figure $F$, showing small femoral epiphysis and mild metaphyseal irregularities in the distal femora ${ }^{228}$, Figure retrieved from the University of Zurich Open Repository and Archive. H) Female patient at the age of 10 years, showing severely disproportionate short stature, with compound heterozygous RMRP mutations 195C $\rightarrow T$ and 254_263delCTCAGCGCGG ${ }^{229}$, copyright clearance received for use of Figure. I) Patient from figure $\mathrm{H}$ : the femur, tibia and fibula are short, with severe metaphyseal changes. The knee epiphyses are small and flattened and there is mesomelic (middle parts of the limbs are disproportionately short) shortening in the upper limbs ${ }^{229}$, copyright clearance received for use of Figure.

CHH mutations may lead to RMRP promoter inefficiency and RNA transcript instability ${ }^{230}$. The patient depicted in Figure 10 C-E was found to have the following compound heterozygous mutations in the RMRP gene: $14 G \rightarrow A$ and 90_91AG $\rightarrow G C^{224}$. RT-qPCR revealed a significant decrease in $R M R P$ expression of the $14 \mathrm{G} \rightarrow \mathrm{A}$ mutant allele. Since the rate of growth depends on the synthesis of proteins, and the synthesis of proteins depends on ribosomes, the regulation of growth must ultimately depend on the regulation of the synthesis of new ribosomes ${ }^{231}$. In concert with $\mathrm{CHH}$ being classified as a ribosomopathy, increased expression levels of uncleaved pre-5.8S rRNA bound to ITS-1 were observed in the patient's fibroblasts and lymphocytes, indicating defective rRNA processing ${ }^{224}$. These pathological observations could be rescued in fibroblasts by the introduction of a wild-type $R M R P^{224}$. However it remains to be determined how $\mathrm{CHH}$-pathological mutations in the RMRP gene lead to the severe dwarfism phenotype observed in $\mathrm{CHH}$. It is expected that highly proliferative growth plates, populated with growth plate chondrocytes (refer to Figure 1), are particularly depending and ribosomal activity and their development may thus be pathologically hampered in $\mathrm{CHH}$, leading to growth retardation. Alternatively, the processing of other RNA substrates of the RNase MRP complex ${ }^{223}$ may be disturbed as well, and it cannot be excluded that these also contribute to the clinical presentation of $\mathrm{CHH}$. 


\subsection{Aims and outline of this thesis}

The cellular processes in which snoRNAs participate are fundamental processes needed for proper cell function, yet their roles in cell differentiation, homeostasis and disease in general have been poorly investigated. Moreover, in the context of chondrogenic differentiation, cartilage homeostasis and disease, snoRNAs have not been investigated so far. In the work presented in this thesis we therefore investigated the involvement of snoRNAs in several chondrocyte and cartilage facets.

Mutations in the RMRP-gene, encoding the snoRNA component of the RNase MRP complex, are the cause of cartilage-hair hypoplasia ${ }^{224,226}$. Cartilage-hair hypoplasia is associated with severe dwarfism caused by impaired skeletal development. However, it is not clear why mutations in RMRP snoRNA lead to skeletal dysplasia. Since chondrogenic differentiation of the growth plate is required for development of the long bones, we hypothesized that the $R M R P$ snoRNA plays a pivotal role in chondrogenic differentiation. In Chapter 2 we therefore investigated whether RMRP RNA has a role in chondrogenic differentiation, which might explain why $\mathrm{CHH}$ presents with a cartilage dysplasia. RNase MRP has a number of substrate RNAs and from most it is not clear how these substrate RNAs may influence cell biological processes, nor is it known how these substrates may be involved in the development of $\mathrm{CHH}$ or chondrogenic differentiation. One of these substrate RNAs is the mRNA of viperin 220 . Viperin, an abbreviation for Virus Inhibitory Protein, Endoplasmic Reticulum-associated, Interferon (IFN)-inducible, is a protein located in the endoplasmic reticulum ${ }^{232}$ and it is well described for its role as an antiviral protein ${ }^{233}$. Viperin expression has been shown to be increased in Cartilage-hair hypoplasia leukocytes ${ }^{234}$ and following knockdown of RNase MRP subunits ${ }^{220}$. However, its function down-stream of RNase MRP and its potential involvement in the skeletal dysplasia seen in $\mathrm{CHH}$ have not been addressed. In Chapter 3, we hypothesized that viperin functions as a chondrogenic regulator downstream of RNase MRP and the RMRP snoRNA and describe our findings related to viperin function during chondrogenic differentiation. The RMRP snoRNA is one highly specific snoRNA belonging to a small group of non-canonical snoRNAs. The majority of the cell's snoRNA collection however belongs to the canonical snoRNAs involved in posttranscriptional modification of rRNAs. Fine-tuning of the cell's rRNA pool by snoRNA- 
mediated post-transcriptional modifications is believed to determine the modus of ribosome activity and control ribosome translation fidelity. It is expected that this is particularly important in translationally active cells, like growth plate chondrocytes, to accurately and efficiently synthesize their proteins for building the cartilaginous growth plate ECM and support their high speed of proliferation. In Chapter 4 we therefore charted the full spectrum of snoRNAs expressed during different phases of chondrogenic differentiation. As a result from "small RNA" RNA sequencing of samples from chondrogenically differentiating ATDC5 cells, we now hypothesize the involvement of specific snoRNAs in the fine-tuning of rRNAs during chondrogenic differentiating. In osteoarthritis, like in chondrogenic differentiation, the chondrogenic phenotype is actively changing ${ }^{235}$. The development of effective treatments for the age-related disease osteoarthritis and the ability to predict disease progression has been hampered by the lack of biomarkers able to demonstrate the course of the disease. Profiling the expression patterns of snoRNAs in joint aging and osteoarthritis may provide insight in their contribution to joint pathology, their use as diagnostic biomarkers and potential as therapeutic targets. In Chapter $\mathbf{5}$ we determined expression patterns of snoRNAs in murine joint ageing and osteoarthritis and examined them as potential biomarkers. In Chapter 6 and 7 the data described in this thesis are discussed with respect to each other and with outsight to future directions for snoRNA research in cartilaginous tissues. 


\section{References general introduction}

1 Mandal, A. What is Cartilage? News Medical Life Sciences, <https://www.newsmedical.net/health/What-is-Cartilage.aspx> (2017).

2 Buckwalter, J. A. \& Mankin, H. J. Articular cartilage: tissue design and chondrocytematrix interactions. Instr Course Lect 47, 477-486 (1998).

Hasler, E. M., Herzog, W., Wu, J. Z., Muller, W. \& Wyss, U. Articular cartilage biomechanics: theoretical models, material properties, and biosynthetic response. Crit Rev Biomed Eng 27, 415-488 (1999).

4 Poole, A. R. et al. Composition and structure of articular cartilage: a template for tissue repair. Clin Orthop Relat Res, S26-33 (2001).

5 Woolf, A. D. \& Pfleger, B. Burden of major musculoskeletal conditions. Bull World Health Organ 81, 646-656 (2003).

6 Emans, P. J. \& Peterson, L. Developing Insights in Cartilage Repair. (2014).

7 Bahney, C. S. et al. Cellular biology of fracture healing. J Orthop Res 37, 35-50, doi:10.1002/jor.24170 (2019).

8 Park, J. et al. Dual pathways to endochondral osteoblasts: a novel chondrocytederived osteoprogenitor cell identified in hypertrophic cartilage. Biol Open 4, 608621, doi:10.1242/bio.201411031 (2015).

9 Yang, G. et al. Osteogenic fate of hypertrophic chondrocytes. Cell Res 24, 1266-1269, doi:10.1038/cr.2014.111 (2014).

10 Zhou, X. et al. Chondrocytes transdifferentiate into osteoblasts in endochondral bone during development, postnatal growth and fracture healing in mice. PLoS Genet 10, e1004820, doi:10.1371/journal.pgen.1004820 (2014).

11 Mackie, E. J., Ahmed, Y. A., Tatarczuch, L., Chen, K. S. \& Mirams, M. Endochondral ossification: how cartilage is converted into bone in the developing skeleton. Int J Biochem Cell Biol 40, 46-62, doi:10.1016/j.biocel.2007.06.009 (2008).

12 Kronenberg, H. M. Developmental regulation of the growth plate. Nature 423, 332336, doi:10.1038/nature01657 (2003).

13 Leung, V. Y. et al. SOX9 governs differentiation stage-specific gene expression in growth plate chondrocytes via direct concomitant transactivation and repression. PLoS Genet 7, e1002356, doi:10.1371/journal.pgen.1002356 (2011).

Han, Y. \& Lefebvre, V. L-Sox5 and Sox6 drive expression of the aggrecan gene in cartilage by securing binding of Sox9 to a far-upstream enhancer. $\mathrm{Mol} \mathrm{Cell} \mathrm{Biol} \mathrm{28,}$ 4999-5013, doi:10.1128/MCB.00695-08 (2008).

$\mathrm{Li}, \mathrm{F}$. et al. Runx2 contributes to murine Col10a1 gene regulation through direct interaction with its cis-enhancer. J Bone Miner Res 26, 2899-2910, doi:10.1002/jbmr.504 (2011). 
Arnold, M. A. et al. MEF2C transcription factor controls chondrocyte hypertrophy and bone development. Dev Cell 12, 377-389, doi:10.1016/j.devcel.2007.02.004 (2007).

17 Mueller, M. B. \& Tuan, R. S. Functional characterization of hypertrophy in chondrogenesis of human mesenchymal stem cells. Arthritis Rheum 58, 1377-1388, doi:10.1002/art.23370 (2008).

Wolpert, L. Skeleton keys; Growth plate dynamics, <http://www.thescientist.com/?articles.view/articleNo/29664/title/Skeleton-Keys/> (2011). Mohan, S. \& Baylink, D. J. Bone growth factors. Clin Orthop Relat Res, 30-48 (1991). Yang, J., Andre, P., Ye, L. \& Yang, Y. Z. The Hedgehog signalling pathway in bone formation. Int J Oral Sci 7, 73-79, doi:10.1038/ijos.2015.14 (2015).

21 Malcolm, S. \& Goodship, J. Genotype to phenotype. 2nd edn, (BIOS Scientific Publishers; Academic Press, 2001).

22 Guo, J. et al. PTH/PTHrP receptor delays chondrocyte hypertrophy via both Runx2dependent and -independent pathways. Dev Biol 292, 116-128, doi:10.1016/j.ydbio.2005.12.044 (2006).

23 Amizuka, N., Warshawsky, H., Henderson, J. E., Goltzman, D. \& Karaplis, A. C. Parathyroid hormone-related peptide-depleted mice show abnormal epiphyseal cartilage development and altered endochondral bone formation. J Cell Biol 126, 1611-1623 (1994).

24 Schipani, E., Kruse, K. \& Juppner, H. A constitutively active mutant PTH-PTHrP receptor in Jansen-type metaphyseal chondrodysplasia. Science 268, 98-100 (1995).

Hellemans, J. et al. Homozygous mutations in IHH cause acrocapitofemoral dysplasia, an autosomal recessive disorder with cone-shaped epiphyses in hands and hips. Am J Hum Genet 72, 1040-1046 (2003).

26 Thisse, B. \& Thisse, C. Functions and regulations of fibroblast growth factor signaling during embryonic development. Dev Biol 287, 390-402, doi:10.1016/j.ydbio.2005.09.011 (2005).

27 Terkeltaub, R. A. et al. Bone morphogenetic proteins and bFGF exert opposing regulatory effects on PTHrP expression and inorganic pyrophosphate elaboration in immortalized murine endochondral hypertrophic chondrocytes (MCT cells). J Bone Miner Res 13, 931-941, doi:10.1359/jbmr.1998.13.6.931 (1998).

28 Teven, C. M., Farina, E. M., Rivas, J. \& Reid, R. R. Fibroblast growth factor (FGF) signaling in development and skeletal diseases. Genes Dis 1, 199-213, doi:10.1016/j.gendis.2014.09.005 (2014).

29 Dorey, K. \& Amaya, E. FGF signalling: diverse roles during early vertebrate embryogenesis. Development 137, 3731-3742, doi:10.1242/dev.037689 (2010).

30 Dailey, L., Ambrosetti, D., Mansukhani, A. \& Basilico, C. Mechanisms underlying differential responses to FGF signaling. Cytokine Growth Factor Rev 16, 233-247, doi:10.1016/j.cytogfr.2005.01.007 (2005). 
31 Yun, Y. R. et al. Fibroblast growth factors: biology, function, and application for tissue regeneration. J Tissue Eng 2010, 218142, doi:10.4061/2010/218142 (2010).

32 Turner, N. \& Grose, R. Fibroblast growth factor signalling: from development to cancer. Nat Rev Cancer 10, 116-129, doi:10.1038/nrc2780 (2010).

Cohen, M. M., Jr. Achondroplasia, hypochondroplasia and thanatophoric dysplasia: clinically related skeletal dysplasias that are also related at the molecular level. Int J Oral Maxillofac Surg 27, 451-455 (1998).

Aviezer, D., Golembo, M. \& Yayon, A. Fibroblast growth factor receptor-3 as a therapeutic target for Achondroplasia--genetic short limbed dwarfism. Curr Drug Targets 4, 353-365 (2003).

35 Kan, S. H. et al. Genomic screening of fibroblast growth-factor receptor 2 reveals a wide spectrum of mutations in patients with syndromic craniosynostosis. Am J Hum Genet 70, 472-486, doi:10.1086/338758 (2002).

36 Cho, G. S., Choi, S. C. \& Han, J. K. BMP signal attenuates FGF pathway in anteroposterior neural patterning. Biochem Biophys Res Commun 434, 509-515, doi:10.1016/j.bbrc.2013.03.105 (2013).

37 Tirosh-Finkel, L. et al. BMP-mediated inhibition of FGF signaling promotes cardiomyocyte differentiation of anterior heart field progenitors. Development 137, 2989-3000, doi:10.1242/dev.051649 (2010).

38 Pizette, S. \& Niswander, L. BMPs are required at two steps of limb chondrogenesis: formation of prechondrogenic condensations and their differentiation into chondrocytes. Dev Biol 219, 237-249, doi:10.1006/dbio.2000.9610 (2000).

Caron, M. M. et al. Hypertrophic differentiation during chondrogenic differentiation of progenitor cells is stimulated by BMP-2 but suppressed by BMP-7. Osteoarthritis Cartilage 21, 604-613, doi:10.1016/j.joca.2013.01.009 (2013).

40 Coleman, C. M. et al. Growth differentiation factor-5 enhances in vitro mesenchymal stromal cell chondrogenesis and hypertrophy. Stem Cells Dev 22, 1968-1976, doi:10.1089/scd.2012.0282 (2013).

41 ten Dijke, P., Cell Signaling Technology. TGF-B / Smad Signaling Interactive Pathway, $<$ https://www.cellsignal.com/contents/science-cst-pathways-stem-cell-markers/tgfsmad-signaling-interactive-pathway/pathways-tgfb $>$ (2014).

42 Schmierer, B. \& Hill, C. S. TGFbeta-SMAD signal transduction: molecular specificity and functional flexibility. Nat Rev Mol Cell Biol 8, 970-982, doi:10.1038/nrm2297 (2007).

43 Horbelt, D., Denkis, A. \& Knaus, P. A portrait of Transforming Growth Factor beta superfamily signalling: Background matters. Int J Biochem Cell Biol 44, 469-474, doi:10.1016/j.biocel.2011.12.013 (2012).

44 Huang, F. \& Chen, Y. G. Regulation of TGF-beta receptor activity. Cell Biosci 2, 9, doi:10.1186/2045-3701-2-9 (2012). 
Sartori, R., Gregorevic, P. \& Sandri, M. TGFbeta and BMP signaling in skeletal muscle: potential significance for muscle-related disease. Trends Endocrinol Metab 25, 464471, doi:10.1016/j.tem.2014.06.002 (2014).

Javed, A. et al. Structural coupling of Smad and Runx2 for execution of the BMP2 osteogenic signal. J Biol Chem 283, 8412-8422, doi:10.1074/jbc.M705578200 (2008).

47 Wang, R. N. et al. Bone Morphogenetic Protein (BMP) signaling in development and human diseases. Genes Dis 1, 87-105, doi:10.1016/j.gendis.2014.07.005 (2014).

48 Moustakas, A. \& Miyazawa, K. TGF-[beta] in human disease. (Springer, 2013).

49 Bonafe, L. et al. Nosology and classification of genetic skeletal disorders: 2015 revision. Am J Med Genet A 167A, 2869-2892, doi:10.1002/ajmg.a.37365 (2015).

50 Martinez-Glez, V. et al. Identification of a mutation causing deficient BMP1/mTLD proteolytic activity in autosomal recessive osteogenesis imperfecta. Hum Mutat 33, 343-350, doi:10.1002/humu.21647 (2012).

51 Asharani, P. V. et al. Attenuated BMP1 function compromises osteogenesis, leading to bone fragility in humans and zebrafish. Am J Hum Genet 90, 661-674, doi:10.1016/j.ajhg.2012.02.026 (2012).

52 Gordon, M. D. \& Nusse, R. Wnt signaling: multiple pathways, multiple receptors, and multiple transcription factors. J Biol Chem 281, 22429-22433, doi:10.1074/jbc.R600015200 (2006).

53 Logan, C. Y. \& Nusse, R. The Wnt signaling pathway in development and disease. Annu Rev Cell Dev Biol 20, 781-810, doi:10.1146/annurev.cellbio.20.010403.113126 (2004).

54 Veeman, M. T., Axelrod, J. D. \& Moon, R. T. A second canon. Functions and mechanisms of beta-catenin-independent Wnt signaling. Dev Cell 5, 367-377 (2003).

55 van den Bosch, M. H. et al. Canonical Wnt signaling skews TGF-beta signaling in chondrocytes towards signaling via ALK1 and Smad 1/5/8. Cell Signal 26, 951-958, doi:10.1016/j.cellsig.2014.01.021 (2014).

56 MacDonald, B. T., Tamai, K. \& He, X. Wnt/beta-catenin signaling: components, mechanisms, and diseases. Dev Cell 17, 9-26, doi:10.1016/j.devcel.2009.06.016 (2009).

57 Wu, Q. et al. Smurf2 induces degradation of GSK-3beta and upregulates beta-catenin in chondrocytes: a potential mechanism for Smurf2-induced degeneration of articular cartilage. Exp Cell Res 315, 2386-2398, doi:10.1016/j.yexcr.2009.05.019 (2009).

58 Borzi, R. M. et al. Matrix metalloproteinase 13 loss associated with impaired extracellular matrix remodeling disrupts chondrocyte differentiation by concerted effects on multiple regulatory factors. Arthritis Rheum 62, 2370-2381, doi:10.1002/art.27512 (2010).

59 Leijten, J. C. et al. GREM1, FRZB and DKK1 mRNA levels correlate with osteoarthritis and are regulated by osteoarthritis-associated factors. Arthritis Res Ther 15, R126, doi:10.1186/ar4306 (2013). 
60

61

62

64

65

66

67

68

69

70

71

72

73

74

75

Chen, L. et al. The inhibition of EZH2 ameliorates osteoarthritis development through the Wnt/beta-catenin pathway. Sci Rep 6, 29176, doi:10.1038/srep29176 (2016).

Usami, Y., Gunawardena, A. T., Iwamoto, M. \& Enomoto-Iwamoto, M. Wnt signaling in cartilage development and diseases: lessons from animal studies. Lab Invest 96, 186-196, doi:10.1038/labinvest.2015.142 (2016).

2 Studer, D., Millan, C., Ozturk, E., Maniura-Weber, K. \& Zenobi-Wong, M. Molecular and biophysical mechanisms regulating hypertrophic differentiation in chondrocytes and mesenchymal stem cells. Eur Cell Mater 24, 118-135; discussion 135 (2012).

Takada, S. et al. Wnt-3a regulates somite and tailbud formation in the mouse embryo. Genes Dev 8, 174-189 (1994).

64 Niemann, S. et al. Homozygous WNT3 mutation causes tetra-amelia in a large consanguineous family. Am J Hum Genet 74, 558-563, doi:10.1086/382196 (2004).

65 Faqeih, E., Shaheen, R. \& Alkuraya, F. S. WNT1 mutation with recessive osteogenesis imperfecta and profound neurological phenotype. J Med Genet 50, 491-492, doi:10.1136/jmedgenet-2013-101750 (2013).

6 Laine, C. M. et al. WNT1 mutations in early-onset osteoporosis and osteogenesis imperfecta. N Engl J Med 368, 1809-1816, doi:10.1056/NEJMoa1215458 (2013).

6 Pyott, S. M. et al. WNT1 mutations in families affected by moderately severe and progressive recessive osteogenesis imperfecta. Am J Hum Genet 92, 590-597, doi:10.1016/j.ajhg.2013.02.009 (2013).

68 Fahiminiya, S. et al. Mutations in WNT1 are a cause of osteogenesis imperfecta. J Med Genet 50, 345-348, doi:10.1136/jmedgenet-2013-101567 (2013).

69 Church, V., Nohno, T., Linker, C., Marcelle, C. \& Francis-West, P. Wnt regulation of chondrocyte differentiation. J Cell Sci 115, 4809-4818, doi:10.1242/jcs.00152 (2002).

Enomoto-Iwamoto, M. et al. The Wnt antagonist Frzb-1 regulates chondrocyte maturation and long bone development during limb skeletogenesis. Dev Biol 251, 142-156, doi:10.1006/dbio.2002.0802 (2002).

71 Rudnicki, J. A. \& Brown, A. M. Inhibition of chondrogenesis by Wnt gene expression in vivo and in vitro. Dev Biol 185, 104-118, doi:10.1006/dbio.1997.8536 (1997).

72 Hartmann, C. \& Tabin, C. J. Wnt-14 plays a pivotal role in inducing synovial joint formation in the developing appendicular skeleton. Cell 104, 341-351, doi:10.1016/s0092-8674(01)00222-7 (2001).

73 Tamamura, Y. et al. Developmental regulation of $\mathrm{Wnt} /$ beta-catenin signals is required for growth plate assembly, cartilage integrity, and endochondral ossification. J Biol Chem 280, 19185-19195, doi:10.1074/jbc.M414275200 (2005).

Guo, X. et al. Wnt/beta-catenin signaling is sufficient and necessary for synovial joint formation. Genes Dev 18, 2404-2417, doi:10.1101/gad.1230704 (2004).

Akiyama, H. et al. Interactions between Sox9 and beta-catenin control chondrocyte differentiation. Genes Dev 18, 1072-1087, doi:10.1101/gad.1171104 (2004). 
76 Zhong, Z. A. et al. Wntless spatially regulates bone development through betacatenin-dependent and independent mechanisms. Dev Dyn 244, 1347-1355, doi:10.1002/dvdy.24316 (2015).

77 Zhu, X. et al. Wls-mediated Wnts differentially regulate distal limb patterning and tissue morphogenesis. Dev Biol 365, 328-338, doi:10.1016/j.ydbio.2012.02.019 (2012).

78 Gao, B. et al. Wnt signaling gradients establish planar cell polarity by inducing Vangl2 phosphorylation through Ror2. Dev Cell 20, 163-176, doi:10.1016/j.devcel.2011.01.001 (2011).

79 Dao, D. Y. et al. Cartilage-specific beta-catenin signaling regulates chondrocyte maturation, generation of ossification centers, and perichondrial bone formation during skeletal development. J Bone Miner Res 27, 1680-1694, doi:10.1002/jbmr.1639 (2012).

80 Hill, T. P., Spater, D., Taketo, M. M., Birchmeier, W. \& Hartmann, C. Canonical Wnt/beta-catenin signaling prevents osteoblasts from differentiating into chondrocytes. Dev Cell 8, 727-738, doi:10.1016/j.devcel.2005.02.013 (2005).

$81 \mathrm{Hu}, \mathrm{H}$. et al. Sequential roles of Hedgehog and Wnt signaling in osteoblast development. Development 132, 49-60, doi:10.1242/dev.01564 (2005).

82 Day, T. F., Guo, X., Garrett-Beal, L. \& Yang, Y. Wnt/beta-catenin signaling in mesenchymal progenitors controls osteoblast and chondrocyte differentiation during vertebrate skeletogenesis. Dev Cell 8, 739-750, doi:10.1016/j.devcel.2005.03.016 (2005).

83 Mak, K. K., Chen, M. H., Day, T. F., Chuang, P. T. \& Yang, Y. Wnt/beta-catenin signaling interacts differentially with Ihh signaling in controlling endochondral bone and synovial joint formation. Development 133, 3695-3707, doi:10.1242/dev.02546 (2006).

84 Chen, M. et al. Inhibition of beta-catenin signaling causes defects in postnatal cartilage development. J Cell Sci 121, 1455-1465, doi:10.1242/jcs.020362 (2008).

Yang, Y., Topol, L., Lee, H. \& Wu, J. Wnt5a and Wnt5b exhibit distinct activities in coordinating chondrocyte proliferation and differentiation. Development 130, 10031015 (2003).

Grumolato, L. et al. Canonical and noncanonical Wnts use a common mechanism to activate completely unrelated coreceptors. Genes Dev 24, 2517-2530, doi:10.1101/gad.1957710 (2010).

87 Oishi, l. et al. The receptor tyrosine kinase Ror2 is involved in non-canonical Wnt5a/JNK signalling pathway. Genes Cells 8, 645-654 (2003).

88 Roifman, $M$. et al. De novo WNT5A-associated autosomal dominant Robinow syndrome suggests specificity of genotype and phenotype. Clin Genet 87, 34-41, doi:10.1111/cge.12401 (2015). 
Bacchelli, C., Wilson, L. C., Cook, J. A., Winter, R. M. \& Goodman, F. R. ROR2 is mutated in hereditary brachydactyly with nail dysplasia, but not in Sorsby syndrome. Clin Genet 64, 263-265, doi:10.1034/j.1399-0004.2003.00139.x (2003).

Samsa, W. E., Zhou, X. \& Zhou, G. Signaling pathways regulating cartilage growth plate formation and activity. Semin Cell Dev Biol 62, 3-15, doi:10.1016/j.semcdb.2016.07.008 (2017).

91 Rinderknecht, E. \& Humbel, R. E. The amino acid sequence of human insulin-like growth factor I and its structural homology with proinsulin. J Biol Chem 253, 27692776 (1978).

92 Denley, A. et al. Structural and functional characteristics of the Val44Met insulin-like growth factor I missense mutation: correlation with effects on growth and development. Mol Endocrinol 19, 711-721, doi:10.1210/me.2004-0409 (2005).

93 Walenkamp, M. J. et al. Homozygous and heterozygous expression of a novel insulinlike growth factor-I mutation. J Clin Endocrinol Metab 90, 2855-2864, doi:10.1210/jc.2004-1254 (2005).

94 Woods, K. A., Camacho-Hubner, C., Barter, D., Clark, A. J. \& Savage, M. O. Insulin-like growth factor I gene deletion causing intrauterine growth retardation and severe short stature. Acta Paediatr Suppl 423, 39-45 (1997).

Caverzasio, J., Montessuit, C. \& Bonjour, J. P. Stimulatory effect of insulin-like growth factor-1 on renal Pi transport and plasma 1,25-dihydroxyvitamin D3. Endocrinology 127, 453-459, doi:10.1210/endo-127-1-453 (1990).

96 Caverzasio, J. \& Bonjour, J. P. Insulin-like growth factor I stimulates Na-dependent Pi transport in cultured kidney cells. Am J Physiol 257, F712-717, doi:10.1152/ajprenal.1989.257.5.F712 (1989).

97 Wang, Y. et al. Insulin-like growth factor-I is essential for embryonic bone development. Endocrinology 147, 4753-4761, doi:10.1210/en.2006-0196 (2006).

98 Laviola, L., Natalicchio, A., Perrini, S. \& Giorgino, F. Abnormalities of IGF-I signaling in the pathogenesis of diseases of the bone, brain, and fetoplacental unit in humans. Am J Physiol Endocrinol Metab 295, E991-999, doi:10.1152/ajpendo.90452.2008 (2008).

99 Li, Y., Wei, X., Zhou, J. \& Wei, L. The age-related changes in cartilage and osteoarthritis. Biomed Res Int 2013, 916530, doi:10.1155/2013/916530 (2013).

100 Felson, D. T. et al. Osteoarthritis: new insights. Part 1: the disease and its risk factors. Ann Intern Med 133, 635-646 (2000).

101 Blagojevic, M., Jinks, C., Jeffery, A. \& Jordan, K. P. Risk factors for onset of osteoarthritis of the knee in older adults: a systematic review and meta-analysis. Osteoarthritis Cartilage 18, 24-33, doi:10.1016/j.joca.2009.08.010 (2010).

102 Loeser, R. F., Goldring, S. R., Scanzello, C. R. \& Goldring, M. B. Osteoarthritis: a disease of the joint as an organ. Arthritis Rheum 64, 1697-1707, doi:10.1002/art.34453 (2012). 
103 MedicineNet. Osteoarthritis, <https://www.medicinenet.com/script/main/art.asp?articlekey=90187> (2016).

104 Andriacchi, T. P. et al. A framework for the in vivo pathomechanics of osteoarthritis at the knee. Ann Biomed Eng 32, 447-457 (2004).

105 Goldring, M. B. \& Marcu, K. B. Cartilage homeostasis in health and rheumatic diseases. Arthritis Res Ther 11, 224, doi:10.1186/ar2592 (2009).

106 Troeberg, L. \& Nagase, H. Proteases involved in cartilage matrix degradation in osteoarthritis. Biochim Biophys Acta 1824, 133-145, doi:10.1016/j.bbapap.2011.06.020 (2012).

107 Ripmeester, E. G. J., Timur, U. T., Caron, M. M. J. \& Welting, T. J. M. Recent Insights into the Contribution of the Changing Hypertrophic Chondrocyte Phenotype in the Development and Progression of Osteoarthritis. Front Bioeng Biotechnol 6, 18, doi:10.3389/fbioe.2018.00018 (2018).

108 Zhu, M. et al. Activation of beta-catenin signaling in articular chondrocytes leads to osteoarthritis-like phenotype in adult beta-catenin conditional activation mice. J Bone Miner Res 24, 12-21, doi:10.1359/jbmr.080901 (2009).

109 Zhu, M. et al. Inhibition of beta-catenin signaling in articular chondrocytes results in articular cartilage destruction. Arthritis Rheum 58, 2053-2064, doi:10.1002/art.23614 (2008).

110 van der Kraan, P. M. \& van den Berg, W. B. Chondrocyte hypertrophy and osteoarthritis: role in initiation and progression of cartilage degeneration? Osteoarthritis Cartilage 20, 223-232, doi:10.1016/j.joca.2011.12.003 (2012).

111 Dong, Y. F., Soung do, Y., Schwarz, E. M., O'Keefe, R. J. \& Drissi, H. Wnt induction of chondrocyte hypertrophy through the Runx2 transcription factor. J Cell Physiol 208, 77-86, doi:10.1002/jcp.20656 (2006).

112 Guo, X., Mak, K. K., Taketo, M. M. \& Yang, Y. The Wnt/beta-catenin pathway interacts differentially with PTHrP signaling to control chondrocyte hypertrophy and final maturation. PLoS One 4, e6067, doi:10.1371/journal.pone.0006067 (2009).

113 Kitagaki, J. et al. Activation of beta-catenin-LEF/TCF signal pathway in chondrocytes stimulates ectopic endochondral ossification. Osteoarthritis Cartilage 11, 36-43 (2003).

114 Gaur, T. et al. Secreted frizzled related protein 1 regulates Wnt signaling for BMP2 induced chondrocyte differentiation. J Cell Physiol 208, 87-96, doi:10.1002/jcp.20637 (2006).

115 Guo, X. \& Wang, X. F. Signaling cross-talk between TGF-beta/BMP and other pathways. Cell Res 19, 71-88, doi:10.1038/cr.2008.302 (2009).

116 Dong, Y. F. et al. Transforming growth factor-beta and Wnt signals regulate chondrocyte differentiation through Twist1 in a stage-specific manner. Mol Endocrinol 21, 2805-2820, doi:10.1210/me.2007-0199 (2007). 
117 Fuentealba, L. C. et al. Integrating patterning signals: Wnt/GSK3 regulates the duration of the BMP/Smad1 signal. Cell 131, 980-993, doi:10.1016/j.cell.2007.09.027 (2007).

118 Huang, B. L., Brugger, S. M. \& Lyons, K. M. Stage-specific control of connective tissue growth factor (CTGF/CCN2) expression in chondrocytes by Sox9 and beta-catenin. J Biol Chem 285, 27702-27712, doi:10.1074/jbc.M110.108498 (2010).

119 Min, J. L. et al. Association of the Frizzled-related protein gene with symptomatic osteoarthritis at multiple sites. Arthritis Rheum 52, 1077-1080, doi:10.1002/art.20993 (2005).

120 Blom, A. B. et al. Involvement of the Wnt signaling pathway in experimental and human osteoarthritis: prominent role of Wnt-induced signaling protein 1. Arthritis Rheum 60, 501-512, doi:10.1002/art.24247 (2009).

121 Wu, Q. et al. Beta-catenin, cartilage, and osteoarthritis. Ann N Y Acad Sci 1192, 344350, doi:10.1111/j.1749-6632.2009.05212.x (2010).

122 van Caam, A. et al. TGFbeta1-induced SMAD2/3 and SMAD1/5 phosphorylation are both ALK5-kinase-dependent in primary chondrocytes and mediated by TAK1 kinase activity. Arthritis Res Ther 19, 112, doi:10.1186/s13075-017-1302-4 (2017).

123 van der Kraan, P. M., Blaney Davidson, E. N. \& van den Berg, W. B. Bone morphogenetic proteins and articular cartilage: To serve and protect or a wolf in sheep clothing's? Osteoarthritis Cartilage 18, 735-741, doi:10.1016/j.joca.2010.03.001 (2010).

124 van der Kraan, P. M. The changing role of TGFbeta in healthy, ageing and osteoarthritic joints. Nat Rev Rheumatol 13, 155-163, doi:10.1038/nrrheum.2016.219 (2017).

125 Tsuchiya, A. et al. Expression of mouse HtrA1 serine protease in normal bone and cartilage and its upregulation in joint cartilage damaged by experimental arthritis. Bone 37, 323-336, doi:10.1016/j.bone.2005.03.015 (2005).

126 Polur, I., Lee, P. L., Servais, J. M., Xu, L. \& Li, Y. Role of HTRA1, a serine protease, in the progression of articular cartilage degeneration. Histol Histopathol 25, 599-608, doi:10.14670/HH-25.599 (2010).

127 Shiga, A. et al. Cerebral small-vessel disease protein HTRA1 controls the amount of TGF-beta1 via cleavage of proTGF-beta1. Hum Mol Genet 20, 1800-1810, doi:10.1093/hmg/ddr063 (2011).

128 Tocharus, J. et al. Developmentally regulated expression of mouse HtrA3 and its role as an inhibitor of TGF-beta signaling. Dev Growth Differ 46, 257-274, doi:10.1111/j.1440-169X.2004.00743.x (2004).

129 Serra, R., Karaplis, A. \& Sohn, P. Parathyroid hormone-related peptide (PTHrP)dependent and -independent effects of transforming growth factor beta (TGF-beta) on endochondral bone formation. J Cell Biol 145, 783-794 (1999).

130 Matsunobu, T. et al. Critical roles of the TGF-beta type I receptor ALK5 in perichondrial formation and function, cartilage integrity, and osteoblast 
differentiation during growth plate development. Dev Biol 332, 325-338, doi:10.1016/j.ydbio.2009.06.002 (2009).

131 Wei, F. et al. Activation of Indian hedgehog promotes chondrocyte hypertrophy and upregulation of MMP-13 in human osteoarthritic cartilage. Osteoarthritis Cartilage 20, 755-763, doi:10.1016/j.joca.2012.03.010 (2012).

132 Lin, A. C. et al. Modulating hedgehog signaling can attenuate the severity of osteoarthritis. Nat Med 15, 1421-1425, doi:10.1038/nm.2055 (2009).

133 Zhou, J. et al. Disrupting the Indian hedgehog signaling pathway in vivo attenuates surgically induced osteoarthritis progression in Col2a1-CreERT2; Ihhfl/fl mice. Arthritis Res Ther 16, R11, doi:10.1186/ar4437 (2014).

134 Ekenstedt, K. J., Sonntag, W. E., Loeser, R. F., Lindgren, B. R. \& Carlson, C. S. Effects of chronic growth hormone and insulin-like growth factor 1 deficiency on osteoarthritis severity in rat knee joints. Arthritis Rheum 54, 3850-3858, doi:10.1002/art.22254 (2006).

135 Martel-Pelletier, J., Di Battista, J. A., Lajeunesse, D. \& Pelletier, J. P. IGF/IGFBP axis in cartilage and bone in osteoarthritis pathogenesis. Inflamm Res 47, 90-100, doi:10.1007/s000110050288 (1998).

136 Bagge, E., Eden, S., Rosen, T. \& Bengtsson, B. A. The prevalence of radiographic osteoarthritis is low in elderly patients with growth hormone deficiency. Acta Endocrinol (Copenh) 129, 296-300 (1993).

137 Bianchi, A. et al. Fibroblast Growth Factor 23 drives MMP13 expression in human osteoarthritic chondrocytes in a Klotho-independent manner. Osteoarthritis Cartilage 24, 1961-1969, doi:10.1016/j.joca.2016.06.003 (2016).

138 Orfanidou, T., Iliopoulos, D., Malizos, K. N. \& Tsezou, A. Involvement of SOX-9 and FGF-23 in RUNX-2 regulation in osteoarthritic chondrocytes. J Cell Mol Med 13, 31863194, doi:10.1111/j.1582-4934.2009.00678.x (2009).

139 Chia, S. L. et al. Fibroblast growth factor 2 is an intrinsic chondroprotective agent that suppresses ADAMTS- 5 and delays cartilage degradation in murine osteoarthritis. Arthritis Rheum 60, 2019-2027, doi:10.1002/art.24654 (2009).

140 Davies, M. R. et al. Ligands for retinoic acid receptors are elevated in osteoarthritis and may contribute to pathologic processes in the osteoarthritic joint. Arthritis Rheum 60, 1722-1732, doi:10.1002/art.24550 (2009).

141 Dreier, R. Hypertrophic differentiation of chondrocytes in osteoarthritis: the developmental aspect of degenerative joint disorders. Arthritis Res Ther 12, 216, doi:10.1186/ar3117 (2010).

142 Stepanov, G. A. et al. Regulatory role of small nucleolar RNAs in human diseases. Biomed Res Int 2015, 206849, doi:10.1155/2015/206849 (2015).

143 Scott, M. S. \& Ono, M. From snoRNA to miRNA: Dual function regulatory non-coding RNAs. Biochimie 93, 1987-1992, doi:10.1016/j.biochi.2011.05.026 (2011).

144 Dieci, G., Preti, M. \& Montanini, B. Eukaryotic snoRNAs: a paradigm for gene expression flexibility. Genomics 94, 83-88, doi:10.1016/j.ygeno.2009.05.002 (2009). 
145 Cooper, G. M. The Cell: A Molecular Approach. 2nd edn, (Sinauer Associates, 2000).

146 Vanzi, F., Vladimirov, S., Knudsen, C. R., Goldman, Y. E. \& Cooperman, B. S. Protein synthesis by single ribosomes. RNA 9, 1174-1179, doi:10.1261/rna.5800303 (2003).

147 Sanchez, C. G. et al. Regulation of Ribosome Biogenesis and Protein Synthesis Controls Germline Stem Cell Differentiation. Cell Stem Cell 18, 276-290, doi:10.1016/j.stem.2015.11.004 (2016).

148 Susor, A., Jansova, D., Anger, M. \& Kubelka, M. Translation in the mammalian oocyte in space and time. Cell Tissue Res 363, 69-84, doi:10.1007/s00441-015-2269-6 (2016).

149 Gregory, B. et al. The small and large ribosomal subunits depend on each other for stability and accumulation. Life Sci Alliance 2, doi:10.26508/Isa.201800150 (2019).

150 Henras, A. K., Plisson-Chastang, C., O'Donohue, M. F., Chakraborty, A. \& Gleizes, P. E. An overview of pre-ribosomal RNA processing in eukaryotes. Wiley Interdiscip Rev RNA 6, 225-242, doi:10.1002/wrna.1269 (2015).

151 Lodish, H., Berk, A., Zipursky, S.L. Molecular Cell Biology. 4th edn, (W.H. Freeman, 2000).

152 Protein synthesis Talking Glossary of Genetic Terms, National Human Genome Research Institute https://wofford-ecs.org/DataAndVisualization/GenomicData/index.htm (2004).

153 Cellular.and.Molecular.Biology. Protein synthesis and ribosomes, <http://www.angelfire.com/sc3/toxchick/celmolbio/celmolbio11.html> (2001).

154 Ladyofhats. Ribosome mRNA translation, $<$ https://en.wikipedia.org/wiki/Translation_(biology)\#/media/File:Ribosome mRNA translation en.svg $>$ (2008).

155 Sirri, V., Grob, A., Berthelet, J., Jourdan, N. \& Roussel, P. Sirtuin 7 promotes 45S prerRNA cleavage at site 2 and determines the processing pathway. J Cell Sci 132, doi:10.1242/jcs.228601 (2019).

156 Popov, A. et al. Duration of the first steps of the human rRNA processing. Nucleus 4, 134-141, doi:10.4161/nucl.23985 (2013).

157 Turowski, T. W. \& Tollervey, D. Transcription by RNA polymerase III: insights into mechanism and regulation. Biochem Soc Trans 44, 1367-1375, doi:10.1042/BST20160062 (2016).

158 Polacek, N. \& Mankin, A. S. The ribosomal peptidyl transferase center: structure, function, evolution, inhibition. Crit Rev Biochem Mol Biol 40, 285-311, doi:10.1080/10409230500326334 (2005).

159 Liang, X. H., Liu, Q. \& Fournier, M. J. Loss of rRNA modifications in the decoding center of the ribosome impairs translation and strongly delays pre-rRNA processing. RNA 15, 1716-1728, doi:10.1261/rna.1724409 (2009).

160 Rodnina, M. V., Daviter, T., Gromadski, K. \& Wintermeyer, W. Structural dynamics of ribosomal RNA during decoding on the ribosome. Biochimie 84, 745-754, doi:10.1016/s0300-9084(02)01409-8 (2002). 
161 Noller, H. F. Biochemical characterization of the ribosomal decoding site. Biochimie 88, 935-941, doi:10.1016/j.biochi.2006.04.006 (2006).

162 Ogle, J. M., Carter, A. P. \& Ramakrishnan, V. Insights into the decoding mechanism from recent ribosome structures. Trends Biochem Sci 28, 259-266, doi:10.1016/S0968-0004(03)00066-5 (2003).

163 Ogle, J. M. \& Ramakrishnan, V. Structural insights into translational fidelity. Annu Rev Biochem 74, 129-177, doi:10.1146/annurev.biochem.74.061903.155440 (2005).

164 Frank, J., Gao, H., Sengupta, J., Gao, N. \& Taylor, D. J. The process of mRNA-tRNA translocation. Proc Natl Acad Sci USA 104, 19671-19678, doi:10.1073/pnas.0708517104 (2007).

165 Kiss, T., Fayet, E., Jady, B. E., Richard, P. \& Weber, M. Biogenesis and intranuclear trafficking of human box C/D and H/ACA RNPs. Cold Spring Harb Symp Quant Biol 71, 407-417, doi:10.1101/sqb.2006.71.025 (2006).

166 Watkins, N. J. \& Bohnsack, M. T. The box C/D and H/ACA snoRNPs: key players in the modification, processing and the dynamic folding of ribosomal RNA. Wiley Interdiscip Rev RNA 3, 397-414, doi:10.1002/wrna.117 (2012).

167 Burman, L. G. \& Mauro, V. P. Analysis of rRNA processing and translation in mammalian cells using a synthetic 18S rRNA expression system. Nucleic Acids Res 40, 8085-8098, doi:10.1093/nar/gks530 (2012).

168 Lygerou, Z., Allmang, C., Tollervey, D. \& Seraphin, B. Accurate processing of a eukaryotic precursor ribosomal RNA by ribonuclease MRP in vitro. Science 272, 268270 (1996).

169 Goldfarb, K. C. \& Cech, T. R. Targeted CRISPR disruption reveals a role for RNase MRP RNA in human preribosomal RNA processing. Genes Dev 31, 59-71, doi:10.1101/gad.286963.116 (2017).

170 Mishra, R. K. \& Eliceiri, G. L. Three small nucleolar RNAs that are involved in ribosomal RNA precursor processing. Proc Natl Acad Sci U S A 94, 4972-4977, doi:10.1073/pnas.94.10.4972 (1997).

171 Kiss, T. Small nucleolar RNAs: an abundant group of noncoding RNAs with diverse cellular functions. Cell 109, 145-148 (2002).

172 Reichow, S. L., Hamma, T., Ferre-D'Amare, A. R. \& Varani, G. The structure and function of small nucleolar ribonucleoproteins. Nucleic Acids Res 35, 1452-1464, doi:10.1093/nar/gkl1172 (2007).

173 Jady, B. E. \& Kiss, T. A small nucleolar guide RNA functions both in 2'-O-ribose methylation and pseudouridylation of the U5 spliceosomal RNA. EMBO J 20, 541551, doi:10.1093/emboj/20.3.541 (2001).

174 Tycowski, K. T., You, Z. H., Graham, P. J. \& Steitz, J. A. Modification of U6 spliceosomal RNA is guided by other small RNAs. Mol Cell 2, 629-638 (1998).

175 Filipowicz, W. \& Pogacic, V. Biogenesis of small nucleolar ribonucleoproteins. Curr Opin Cell Biol 14, 319-327 (2002). 
176 Matera, A. G., Terns, R. M. \& Terns, M. P. Non-coding RNAs: lessons from the small nuclear and small nucleolar RNAs. Nat Rev Mol Cell Biol 8, 209-220, doi:10.1038/nrm2124 (2007).

177 Tafer, H., Kehr, S., Hertel, J., Hofacker, I. L. \& Stadler, P. F. RNAsnoop: efficient target prediction for H/ACA snoRNAs. Bioinformatics 26, 610-616, doi:10.1093/bioinformatics/btp680 (2010).

178 Calore, F., Lovat, F. \& Garofalo, M. Non-coding RNAs and cancer https://www.intechopen.com/books/oncogene-and-cancer-from-bench-to-

clinic/non-coding-rnas-and-cancer. Int J Mol Sci 14, 17085-17110, doi:10.3390/ijms140817085 (2013).

179 Steinbusch, M. M. et al. Serum snoRNAs as biomarkers for joint ageing and post traumatic osteoarthritis. Sci Rep 7, 43558, doi:10.1038/srep43558 (2017).

180 Zhang, L. et al. Serum non-coding RNAs as biomarkers for osteoarthritis progression after ACL injury. Osteoarthritis Cartilage 20, 1631-1637, doi:10.1016/j.joca.2012.08.016 (2012).

181 Liao, J. et al. Small nucleolar RNA signatures as biomarkers for non-small-cell lung cancer. Mol Cancer 9, 198, doi:10.1186/1476-4598-9-198 (2010).

182 Okugawa, Y. et al. Clinical significance of SNORA42 as an oncogene and a prognostic biomarker in colorectal cancer. Gut 66, 107-117, doi:10.1136/gutjnl-2015-309359 (2017).

183 Kishikawa, T. et al. Circulating RNAs as new biomarkers for detecting pancreatic cancer. World J Gastroenterol 21, 8527-8540, doi:10.3748/wjg.v21.i28.8527 (2015).

184 Hunter, M. P. et al. Detection of microRNA expression in human peripheral blood microvesicles. PLoS One 3, e3694, doi:10.1371/journal.pone.0003694 (2008).

185 Enderle, D. et al. Characterization of RNA from Exosomes and Other Extracellular Vesicles Isolated by a Novel Spin Column-Based Method. PLoS One 10, e0136133, doi:10.1371/journal.pone.0136133 (2015).

186 Badhai, J., Frojmark, A. S., E, J. D., Schuster, J. \& Dahl, N. Ribosomal protein S19 and S24 insufficiency cause distinct cell cycle defects in Diamond-Blackfan anemia. Biochim Biophys Acta 1792, 1036-1042, doi:10.1016/j.bbadis.2009.08.002 (2009).

187 Hariharan, N. \& Sussman, M. A. Stressing on the nucleolus in cardiovascular disease. Biochim Biophys Acta 1842, 798-801, doi:10.1016/j.bbadis.2013.09.016 (2014).

188 Trainor, P. A. \& Merrill, A. E. Ribosome biogenesis in skeletal development and the pathogenesis of skeletal disorders. Biochim Biophys Acta 1842, 769-778, doi:10.1016/j.bbadis.2013.11.010 (2014).

189 Bachellerie, J. P., Cavaille, J. \& Huttenhofer, A. The expanding snoRNA world. Biochimie 84, 775-790 (2002).

190 Tollervey, D. \& Kiss, T. Function and synthesis of small nucleolar RNAs. Curr Opin Cell Biol 9, 337-342 (1997). 
191 Gee, H. E. et al. The small-nucleolar RNAs commonly used for microRNA normalisation correlate with tumour pathology and prognosis. Br J Cancer 104, 11681177, doi:10.1038/sj.bjc.6606076 (2011).

192 Mei, Y. P. et al. Small nucleolar RNA 42 acts as an oncogene in lung tumorigenesis. Oncogene 31, 2794-2804, doi:10.1038/onc.2011.449 (2012).

$193 \mathrm{Su}, \mathrm{H}$. et al. Elevated snoRNA biogenesis is essential in breast cancer. Oncogene 33, 1348-1358, doi:10.1038/onc.2013.89 (2014).

194 Belin, S. et al. Dysregulation of ribosome biogenesis and translational capacity is associated with tumor progression of human breast cancer cells. PLoS One 4, e7147, doi:10.1371/journal.pone.0007147 (2009).

195 Ben-Shem, A. et al. The structure of the eukaryotic ribosome at $3.0 \mathrm{~A}$ resolution. Science 334, 1524-1529, doi:10.1126/science.1212642 (2011).

196 Decatur, W. A. \& Fournier, M. J. rRNA modifications and ribosome function. Trends Biochem Sci 27, 344-351, doi:10.1016/s0968-0004(02)02109-6 (2002).

197 Sloan, K. E. et al. Tuning the ribosome: The influence of rRNA modification on eukaryotic ribosome biogenesis and function. RNA Biol 14, 1138-1152, doi:10.1080/15476286.2016.1259781 (2017).

198 Polikanov, Y. S., Melnikov, S. V., Soll, D. \& Steitz, T. A. Structural insights into the role of rRNA modifications in protein synthesis and ribosome assembly. Nat Struct Mol Biol 22, 342-344, doi:10.1038/nsmb.2992 (2015).

199 Lestrade, L. \& Weber, M. J. snoRNA-LBME-db, a comprehensive database of human H/ACA and C/D box snoRNAs. Nucleic Acids Res 34, D158-162, doi:10.1093/nar/gkj002 (2006).

200 Baudin-Baillieu, A. et al. Nucleotide modifications in three functionally important regions of the Saccharomyces cerevisiae ribosome affect translation accuracy. Nucleic Acids Res 37, 7665-7677, doi:10.1093/nar/gkp816 (2009).

201 Marcel, V. et al. p53 acts as a safeguard of translational control by regulating fibrillarin and rRNA methylation in cancer. Cancer Cell 24, 318-330, doi:10.1016/j.ccr.2013.08.013 (2013).

202 Barna, M. et al. Suppression of Myc oncogenic activity by ribosomal protein haploinsufficiency. Nature 456, 971-975, doi:10.1038/nature07449 (2008).

203 King, T. H., Liu, B., McCully, R. R. \& Fournier, M. J. Ribosome structure and activity are altered in cells lacking snoRNPs that form pseudouridines in the peptidyl transferase center. Mol Cell 11, 425-435, doi:10.1016/s1097-2765(03)00040-6 (2003).

204 Erales, J. et al. Evidence for rRNA 2'-O-methylation plasticity: Control of intrinsic translational capabilities of human ribosomes. Proc Natl Acad Sci U S A 114, 1293412939, doi:10.1073/pnas.1707674114 (2017).

205 Penzo, M. et al. Human ribosomes from cells with reduced dyskerin levels are intrinsically altered in translation. FASEB J 29, 3472-3482, doi:10.1096/fj.15-270991 (2015). 
206 Higa-Nakamine, S. et al. Loss of ribosomal RNA modification causes developmental defects in zebrafish. Nucleic Acids Res 40, 391-398, doi:10.1093/nar/gkr700 (2012).

207 Trahan, C. \& Dragon, F. Dyskeratosis congenita mutations in the H/ACA domain of human telomerase RNA affect its assembly into a pre-RNP. RNA 15, 235-243, doi:10.1261/rna.1354009 (2009).

208 Vulliamy, T. J. et al. Mutations in dyskeratosis congenita: their impact on telomere length and the diversity of clinical presentation. Blood 107, 2680-2685, doi:10.1182/blood-2005-07-2622 (2006).

209 Savage, S. A. \& Bertuch, A. A. The genetics and clinical manifestations of telomere biology disorders. Genet Med 12, 753-764, doi:10.1097/GIM.0b013e3181f415b5 (2010).

210 Alter, B. P., Giri, N., Savage, S. A. \& Rosenberg, P. S. Cancer in dyskeratosis congenita. Blood 113, 6549-6557, doi:10.1182/blood-2008-12-192880 (2009).

211 Welting, T. J., van Venrooij, W. J. \& Pruijn, G. J. Mutual interactions between subunits of the human RNase MRP ribonucleoprotein complex. Nucleic Acids Res 32, 2138-2146, doi:10.1093/nar/gkh539 (2004).

212 Chang, D. D. \& Clayton, D. A. A mammalian mitochondrial RNA processing activity contains nucleus-encoded RNA. Science 235, 1178-1184, doi:10.1126/science.2434997 (1987).

213 van Eenennaam, H., Jarrous, N., van Venrooij, W. J. \& Pruijn, G. J. Architecture and function of the human endonucleases RNase P and RNase MRP. IUBMB Life 49, 265272, doi:10.1080/15216540050033113 (2000).

214 Jarrous, N. Human ribonuclease P: subunits, function, and intranuclear localization. RNA 8, 1-7, doi:10.1017/s1355838202011184 (2002).

215 Reddy, R. \& Shimba, S. Structural and functional similarities between MRP and RNase P. Mol Biol Rep 22, 81-85, doi:10.1007/bf00988710 (1995).

216 Forster, A. C. \& Altman, S. Similar cage-shaped structures for the RNA components of all ribonuclease $\mathrm{P}$ and ribonuclease MRP enzymes. Cell 62, 407-409, doi:10.1016/0092-8674(90)90003-w (1990).

217 Pyle, A. M. Ribozymes: a distinct class of metalloenzymes. Science 261, 709-714, doi:10.1126/science.7688142 (1993).

218 Chang, D. D. \& Clayton, D. A. A novel endoribonuclease cleaves at a priming site of mouse mitochondrial DNA replication. EMBO J 6, 409-417 (1987).

219 Gill, T., Cai, T., Aulds, J., Wierzbicki, S. \& Schmitt, M. E. RNase MRP cleaves the CLB2 mRNA to promote cell cycle progression: novel method of mRNA degradation. $\mathrm{Mol}$ Cell Biol 24, 945-953 (2004).

220 Mattijssen, S. et al. Viperin mRNA is a novel target for the human RNase MRP/RNase P endoribonuclease. Cell Mol Life Sci 68, 2469-2480, doi:10.1007/s00018-010-0568-3 (2011).

221 Maida, Y. et al. An RNA-dependent RNA polymerase formed by TERT and the RMRP RNA. Nature 461, 230-235, doi:10.1038/nature08283 (2009). 
222 Welting, T. J. M. Human RNase MRP complexes: The Rpp25-Rpp20 dimer makes the difference. Dissertation (2007).

223 Mattijssen, S., Welting, T. J. \& Pruijn, G. J. RNase MRP and disease. Wiley Interdiscip Rev RNA 1, 102-116, doi:10.1002/wrna.9 (2010).

224 Thiel, C. T. et al. Severely incapacitating mutations in patients with extreme short stature identify RNA-processing endoribonuclease RMRP as an essential cell growth regulator. Am J Hum Genet 77, 795-806, doi:10.1086/497708 (2005).

225 Makitie, O. \& Kostjukovits, S. in GeneReviews(R) (eds R. A. Pagon et al.) (1993).

226 Ridanpaa, M. et al. Mutations in the RNA component of RNase MRP cause a pleiotropic human disease, cartilage-hair hypoplasia. Cell 104, 195-203 (2001).

227 Kuijpers, T. W. et al. Short-limbed dwarfism with bowing, combined immune deficiency, and late onset aplastic anaemia caused by novel mutations in the RMPR gene. J Med Genet 40, 761-766 (2003).

228 Turkkani-Asal, G. et al. Mild clinical phenotype and subtle radiographic findings in an infant with cartilage-hair hypoplasia https://www.zora.uzh.ch/id/eprint/31672/. Turk J Pediatr 51, 493-496 (2009).

229 Thiel, C. T., Mortier, G., Kaitila, I., Reis, A. \& Rauch, A. Type and level of RMRP functional impairment predicts phenotype in the cartilage hair hypoplasia-anauxetic dysplasia spectrum. Am J Hum Genet 81, 519-529, doi:10.1086/521034 (2007).

230 Nakashima, E. et al. Cartilage hair hypoplasia mutations that lead to RMRP promoter inefficiency or RNA transcript instability. Am J Med Genet A 143A, 2675-2681, doi:10.1002/ajmg.a.32053 (2007).

231 Conlon, I. \& Raff, M. Control and maintenance of mammalian cell size: response. BMC Cell Biol 5, 36, doi:10.1186/1471-2121-5-36 (2004).

232 Hinson, E. R. \& Cresswell, P. The antiviral protein, viperin, localizes to lipid droplets via its N-terminal amphipathic alpha-helix. Proc Natl Acad Sci U S A 106, 2045220457, doi:10.1073/pnas.0911679106 (2009).

233 Mattijssen, S. \& Pruijn, G. J. Viperin, a key player in the antiviral response. Microbes Infect 14, 419-426, doi:10.1016/j.micinf.2011.11.015 (2012).

234 Hermanns, P. et al. Consequences of mutations in the non-coding RMRP RNA in cartilage-hair hypoplasia. Hum Mol Genet 14, 3723-3740, doi:10.1093/hmg/ddi403 (2005).

235 Le, L. T., Swingler, T. E. \& Clark, I. M. Review: the role of microRNAs in osteoarthritis and chondrogenesis. Arthritis Rheum 65, 1963-1974, doi:10.1002/art.37990 (2013). 
General introduction 
1 Laboratory for Experimental Orthopedics, Department of Orthopedic Surgery, Maastricht University Medical Centre, Maastricht, the Netherlands

2 Department of Pediatrics, Medical Center - University of Freiburg, Faculty of Medicine, University of Freiburg, Freiburg, Germany

${ }^{3}$ Department of Biomolecular Chemistry, Institute for Molecules and Materials and Radboud Institute for Molecular Life Sciences, Radboud University, Nijmegen, the Netherlands

${ }^{4}$ Center for Heart Failure Research, Department of Cardiology, CARIM School for Cardiovascular Diseases, Maastricht University, Maastricht, the Netherlands

${ }^{5}$ Medical Faculty, Otto-von-Guericke-University of Magdeburg, Magdeburg, Germany 


\section{Chapter 2}

\section{Expression of RMRP RNA is regulated in chondrocyte hypertrophy and determines chondrogenic differentiation}

Mandy M.F. Steinbusch', Marjolein M.J. Caron', Don A.M. Surtel', Franziska Friedrich², Ekkehart Lausch², Ger J.M. Pruijn ${ }^{3}$, Wouter Verhesen4, Blanche L.M. Schroen ${ }^{4}$, Lodewijk W. van Rhijn', Bernhard Zabel ${ }^{5}$, Tim J.M. Welting'

Published in Nature Scientific Reports: Sci Rep 7 (2017), 6440, doi: 10.1038/s41598-017-06809-5 


\section{Summary}

Mutations in the RMRP-gene, encoding the IncRNA component of the RNase MRP complex, are the origin of cartilage-hair hypoplasia. Cartilage-hair hypoplasia is associated with severe dwarfism caused by impaired skeletal development. However, it is not clear why mutations in RMRP RNA lead to skeletal dysplasia. Since chondrogenic differentiation of the growth plate is required for development of long bones, we hypothesized that RMRP RNA plays a pivotal role in chondrogenic differentiation. Expression of Rmrp RNA and RNase MRP protein subunits was detected in the murine growth plate and during the course of chondrogenic differentiation of ATDC5 cultures, where Rmrp RNA expression was found to be correlated with chondrocyte hypertrophy. Genetic interference with Rmrp RNA expression in ATDC5 cultures caused a deregulation of chondrogenic differentiation, with a prominent impact on hypertrophy

and changes in pre-rRNA processing and rRNA levels. Promoter reporter studies showed that Rmrp RNA expression responds to chondrogenic morphogens. Chondrogenic transdifferentiation of cartilage-hair hypoplasia fibroblasts was impaired with a pronounced impact on hypertrophic differentiation. Together, our data show that RMRP RNA expression is regulated during different stages of chondrogenic differentiation and indicate that $R M R P$ RNA may play a pivotal role in chondrocyte hypertrophy, with potential consequences for CHH pathobiology. 


\section{Introduction}

RNase MRP is a small nucleolar ribonucleoprotein (snoRNP) particle, consisting of the $R M R P$ long non-coding RNA (IncRNA) and several protein subunits (i.e. Rpp14, Rpp20, Rpp21, Rpp25, Rpp30, Rpp38, Rpp40, hPop1, hPop4 and hPop5 ${ }^{1}$ ). RNase MRP is ubiquitously present in eukaryotes and acts as an endoribonuclease which cleaves several RNA substrates. It has been reported to be involved in mitochondrial DNA replication ${ }^{2}$, cleaves pre-rRNA in the internal transcribed spacer 1 (ITS1) $)^{3,4}$, plays a role in cell cycle regulation by cleaving cyclin b2 $\mathrm{mRNA}^{5}$, and is able to cleave viperin $\mathrm{mRNA}^{6}$. In addition, it has been shown that RMRP RNA and the telomerase-associated reverse transcriptase (TERT) protein are able to form a complex that exerts RNA-dependent RNA polymerase activity, which is able to generate a double stranded RMRP RNA molecule. Via a Dicer-dependent route siRNAs are generated from this molecule, downregulating cellular $R M R P$ levels and providing a negative feedback mechanism controlling $R M R P$ RNA levels ${ }^{7}$. In addition, $R M R P$ RNA is the source of at least two other short RNAs designated RMRP-S1 and RMRP-S2, which function as miRNAs and have gene-silencing activity relevant for human cartilage-hair hypoplasia $(\mathrm{CHH})^{8}$. Recently, RMRP RNA has been shown to interact with DDX5 and play a role in RORyt-dependent TH17 biology ${ }^{9}$. RNase MRP is structurally and functionally related to the RNase $\mathrm{P}$ endoribonuclease, which has a unique RNA component, but shares many protein subunits with RNase MRP ${ }^{10}$. RNase $\mathrm{P}$ is critically involved in maturation of tRNAs by cleaving the $5^{\prime}$ leader of pre-tRNA and is involved in RNA polymerase I and III transcriptional activity $^{11-13}$.

Mutations in the RMRP gene are the cause of a severe form of dwarfism known as the cartilage-hair hypoplasia ${ }^{14}$ (CHH: OMIM \#250250) - anauxetic dysplasia ${ }^{15}$ (AAD: OMIM \#607095) spectrum of disorders ${ }^{16}$. $\mathrm{CHH}$ is also termed metaphyseal chondrodysplasia, McKusick-type ${ }^{17}$. To date, more than hundred individual $\mathrm{CHH}$-pathogenic mutations have been identified in the RMRP gene ${ }^{14}$. These mutations are located either in the transcribed region or in the proximal promoter region, often between the TATA box and the transcription start site. Promoter mutations may result in inefficient transcription of the $R M R P$ gene, whereas mutations in the transcribed region are thought to influence RNase MRP complex assembly, enzymatic activity, subcellular localization, substrate recognition or RNA stability ${ }^{14}$. 
One phenotypic hallmark of $\mathrm{CHH}$ is short-limbed dwarfism accompanied by abnormal growth plate development. Other symptoms include sparse thin hair, anaemia, Hirschsprung's disease, bronchiectasis, and impaired T-cell immunity. In addition, adult patients have a predisposition to certain cancers (i.e. squamous cell carcinoma, basal cell carcinoma and non-Hodgkin lymphoma) ${ }^{16,18}$. Longitudinal bone growth during skeletal development and lengthening of the limbs depends on endochondral ossification. Endochondral ossification is a multistage process in the growth plates of developing long bones. Growth plates are populated by highly proliferative chondrocytes, which differentiate into mineralizing hypertrophic chondrocytes that either die from apoptosis or transdifferentiate into osteoblasts ${ }^{19-21}$. The remaining mineralized extracellular matrix (ECM) provides a scaffold for osteoblasts and osteoclasts to adhere and remodel, setting the stage for bone apposition and thus longitudinal bone growth and limb development ${ }^{22,23}$. In the proliferative zone of the growth plate, Sox9 drives the expression of type II collagen (Col2a1), the main marker for this stage of chondrogenic differentiation. Runx2 and Mef2c drive the expression of type $X$ collagen (Col10a1) during the hypertrophic phase of chondrogenic differentiation. Many skeletal abnormalities have been described that are often caused by mutations in genes involved in growth plate development ${ }^{24}$.

Although the involvement of the RMRP RNA in the molecular pathology of $\mathrm{CHH}$ has been recognized, its role in growth plate biology remains elusive. As abnormal growth plate development is one of the radiological characteristics of $\mathrm{CHH}^{25}$, and chondrogenic differentiation in the growth plates is required for skeletal development ${ }^{23}$, we hypothesized that the RMRP IncRNA may also function as an important regulator of chondrogenic differentiation. 


\section{Materials and methods}

\section{In situ hybridization (ISH)}

Decalcified knee epihyseal growth plates of 6-week-old C57BL/6 mice (use of mouse growth plates was approved by the Maastricht University Animal Ethics Committee, according to Dutch law; and methods utilized to obtain growth plates were carried out in accordance with Maastricht University Animal Ethics Committee and Dutch law) were embedded in paraffin and $5 \mu \mathrm{m}$ sections were cut. Sections were deparaffinized in a xylene / ethanol series ending in PBS (136 mM NaCl (Merck Millipore, Darmstadt, Germany), $2.7 \mathrm{mM} \mathrm{KCl}$

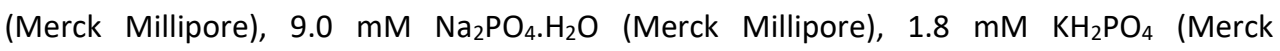
Millipore)). Antigen retrieval was performed using $20 \mu \mathrm{g} / \mathrm{ml}$ proteinase $\mathrm{K}$ (Exiqon, Vedbæk, Denmark). Slides were washed extensively with demineralized water to remove proteinase K. Slides were pre-hybridized with microRNA ISH buffer (Exiqon; miRCURY LNA microRNA ISH optimization kit FFPE). Hybridization was performed for 1 hour at $50^{\circ} \mathrm{C}$ on a heating plate using $80 \mathrm{nM}$ of Rmrp RNA double-digoxigenin (DIG)-labeled miRCURY LNA probe (5'DIG-CTGACGGATGACGC-3'DIG; custom ordered at Exiqon) or a double-DIG labeled scrambled LNA miRNA probe (5'-GTGTAACACGTCTATACGCCCA-3'; Exiqon) in miRNA ISH buffer. Slides were washed three times with $5 x \operatorname{SSC}(0.75 \mathrm{M} \mathrm{NaCl}, 0.075 \mathrm{M}$ sodium citrate) for 10 minutes at $50^{\circ} \mathrm{C}$. Subsequently slides were blocked with blocking solution (DIG Wash and Block buffer set; Roche, Basel, Switzerland) for 30 minutes and incubated with anti-DIGAlkaline Phosphatase (AP) Fab fragments (1:500; Roche) in blocking solution for 1 hour. Endogenous AP was blocked with Levamisole solution (Vector Laboratories, Burlingame, CA, USA) and bound anti-DIG-AP Fab fragments were detected with AP substrate (NBT/BCIP; Roche) in demineralized water. Slides were washed in PBS-T (0.1\% Tween 20; Sigma-Aldrich, St. Louis, MO, USA), counter-stained with $0.01 \%$ FastGreen (Sigma-Aldrich), dehydrated and mounted with Entellan (Merck Millipore). 


\section{Immunohistochemistry (IHC)}

Knee joint epiphyseal growth plates of 6-week-old C57BL/6 mice were formalin-fixed and decalcified using $0.5 \mathrm{M}$ ethylenediaminetetraacetic acid (EDTA; VWR Prolabo, Amsterdam, the Netherlands), $\mathrm{pH}$ 7.8. After paraffin-embedding $5 \mu \mathrm{m}$ tissue sections were cut. Sections were deparaffinized in a xylene / ethanol series ending in PBS. For antigen retrieval (COL10A1 detection) sections were treated with $0.4 \%$ hyaluronidase (Sigma-Aldrich) at $37^{\circ} \mathrm{C}$ for 30 minutes. Sections for detection of RNase MRP protein subunits were incubated in hot citrate buffer (1.8 mM citric acid (Sigma-Aldrich) and $8.2 \mathrm{mM}$ tri-sodium citrate (VWR Prolabo)) for 30 minutes. Endogenous peroxidase activity was inactivated using peroxidaseblocking solution (Dako, Troy, MI, USA). Next, slides were blocked with $10 \%$ normal sheep serum in PBS-T. Primary antibodies were incubated for 1 hour at room temperature. Rabbit polyclonal anti-COL10A1 ${ }^{26}$ was used at a 1:1,000 dilution. Rabbit polyclonal anti-Rpp2527, anti-Rpp30 ${ }^{28}$, anti-Rpp38 ${ }^{28}$, anti-Rpp40 ${ }^{29}$ and anti-hPop ${ }^{30}$ were used at a 1:100 dilution. Bound antibodies were detected with horseradish peroxidase (HRP)-conjugated anti-rabbit secondary antibodies (Dako EnVision) for 30 minutes at room temperature. DAB chromogen substrate (Dako) was used for detection. Sections were counterstained with haematoxylin (Dako), dehydrated and mounted with histomount (Thermo Shandon, Waltham, MA, USA). Microscopic images were acquired using a Zeiss Axioscope A1.

\section{Cell culture and differentiation of ATDC5 and MCT cells and siRNA transfection}

ATDC5 cells ${ }^{31}$ were cultured in a humidified atmosphere at $37^{\circ} \mathrm{C}, 5 \% \mathrm{CO}_{2}$ in proliferation medium (DMEM/F12 (Invitrogen, Carlsbad, CA, USA), 5\% FCS (PAA, Pasching, Austria), 1\% antibiotic/antimycotic (Invitrogen), 1\% NEAA (Invitrogen)). To induce chondrogenic differentiation, cells were plated at 6,400 cells $/ \mathrm{cm}^{2}$. After 24 hours chondrogenic differentiation was initiated by changing the medium to differentiation medium (proliferation medium supplemented with $10 \mu \mathrm{g} / \mathrm{ml}$ insulin (Sigma-Aldrich), $10 \mu \mathrm{g} / \mathrm{ml}$ transferrin (Roche), $30 \mathrm{nM}$ sodium selenite (Sigma-Aldrich)). Differentiation medium was refreshed every two days for the first 10 days, and each day after day 10. Chondrogenically differentiating ATDC5 cells were transfected (Icafectin; Eurogentec, Seraing, Belgium) with 
100 nM siRNA duplexes (custom synthesized by Eurogentec, see Table 1) targeting Sox9, Bapx1 or Rmrp RNA on day $-1,2$, and 5 during differentiation in the presence or absence of 30 nM BMP-2 (Life Technologies, Carlsbad, CA, USA). Scrambled siRNA duplex was purchased from Eurogentec (REF: SR-CL000-005). Cells were harvested for RNA isolation on day 0, 7 and 10 during differentiation (TRIzol reagent; Invitrogen) and samples were kept at $-80^{\circ} \mathrm{C}$ until further analysis. In addition, ATDC5 cells were differentiated for 14 days and exposed to 5 or $50 \mathrm{nM}$ PTHrP (Bachem, Bubendorf, Switzerland) from day 10 onwards (medium with PTHrP was refreshed on day 12 during chondrogenic differentiation). MCT cells $\mathrm{s}^{32}$ were cultured in a humidified atmosphere at the permissive temperature of $32^{\circ} \mathrm{C}, 8 \%$ $\mathrm{CO}_{2}$ in proliferation medium (DMEM/F12 (Invitrogen), 10\% FCS (PAA), 1\% antibiotic/antimycotic (Invitrogen), 1\% NEAA (Invitrogen)). Hypertrophic differentiation was induced in the absence or presence of $50 \mathrm{nM}$ PTHrP (Bachem) by switching culture conditions to $37^{\circ} \mathrm{C}, 5 \% \mathrm{CO}_{2}$ for 24 hours. Cells were harvested for RNA isolation (TRIzol reagent; Invitrogen) and samples were kept at $-80^{\circ} \mathrm{C}$ until further analysis. To maintain $R m r p$ knockdown during an extended period of time during chondrogenic differentiation, scrambled (Eurogentec SR-CLO00-005) and Rmrp RNA (Table 1) siRNAs were either transfected on day 2 and 5 of ATDC5 chondrogenic differentiation for read-out at day 7 or on day 2, 5 and 8 in chondrogenic differentiation for read-out at day 14 .

\section{Table 1. siRNA sequences}

\begin{tabular}{|l|l|l|}
\hline siRNA & Sense & Anti-sense \\
\hline siSox9 & GACUCACAUCUCUCCUAAUTT & AUUAGGAGAGAUGUGAGUCTT \\
\hline siBapx1 & CAGAGACGCAAGUGAAGAUTT & AUCUUCACUUGCGUCUCUGTT \\
\hline siRmrp RNA & CAUGUUCCUUAUCCUUUCGTT & CGAAAGGAUAAGGAACAUGTT \\
\hline
\end{tabular}

\section{Description of plasmids, transfection and bioluminescence detection}

Plasmid constructs were generated using traditional cloning methods and cloned inserts were verified by DNA sequencing. The mouse 1500 nucleotide sequence upstream of the mouse Rmrp transcription start site was amplified from ATDC5 genomic DNA and cloned into the pGluc-Basic vector (NEB) using primer-introduced restriction sites EcoRV and HindIII 
(pGluc-Rmrp-prom). As a transfection control the pGL4.20[luc2/Puro] (Promega, Southampton, UK) vector was used in which the CMV promoter was cloned (pGL4.20[luc2/Puro]-CMV). Day 5-differentiating ATDC5 cells in 12 well plates were transfected with 500 ng pGluc-Rmrp-prom and 125 ng pGL4.20[luc2/Puro]-CMV using 1 $\mu \mathrm{g} / \mu \mathrm{l}$ polyethylenimine (PEI; Polysciences, Warrington, $\mathrm{PA}, \mathrm{USA}$ ) transfection reagent. DNA and PEI were complexed for 10 minutes at room temperature in DMEM/F12 (1.9 $\mu \mathrm{l} \mathrm{PEI} \mathrm{per}$ $625 \mathrm{ng}$ construct per well) and added to the ATDC5 cells. Five hours post-transfection medium was supplemented with BMP-2 (30 ng/ml; Life Technologies), PTHrP (100 nM; Bachem), TGF- $\beta 3$ (10 ng/ml; Life Technologies), dorsomorphin (10 $\mu \mathrm{M}$; Santa Cruz Biotechnology, Dallas, TX, USA), (5Z)-7-Oxozeaenol (0.5 $\mu \mathrm{M}$; Merck Millipore), SHH (150 ng/ml; R\&D systems, Minneapolis, MN, USA), IHH (150 ng/ml; R\&D systems), WNT5A (15 ng/ml; R\&D systems), WNT3A (15 ng/ml; Peprotech, Rocky Hill, NJ, USA), GDF-5 (100 ng/ml; Peprotech) and bFGF (15 ng/ml; Peprotech). After 36 hours samples were harvested for bioluminescence detection using the Dual Luciferase reporter assay system (Promega) as described by the manufacturer and measured on a Fluostar Omega plate reader (BMG Labtech, Ortenberg, Germany).

\section{RT-qPCR}

RNA was isolated from TRIzol samples by collecting the aqueous phase after phase separation. RNA was precipitated with isopropanol $\left(30\right.$ minutes, $\left.-80^{\circ} \mathrm{C}\right)$ and centrifuged for 30 minutes at $20,000 \times \mathrm{g}, 4^{\circ} \mathrm{C}$. RNA pellets were washed with $80 \%$ ethanol and potential DNA contamination was removed by DNase I (Roche) treatment ( 1 hour, $37^{\circ} \mathrm{C}$ ). After subsequent ethanol precipitation, RNA was dissolved in $15 \mu \mathrm{L}$ DNase/RNase free water (Eurogentec). RNA quantity and purity were determined spectrophotometrically (Biodrop, Isogen Life Sciences, Utrecht, the Netherlands). DNA-free total RNA was reverse transcribed using standard procedures and random hexamer priming as described previously ${ }^{33}$. Real time quantitative PCR (RT-qPCR) was performed in 96-well optical plates. For each cDNA sample a mix was prepared consisting of Mesagreen qPCR Mastermix Plus for SYBR Green (Eurogentec) and $300 \mathrm{nM}$ forward and reverse oligonucleotides. Serially diluted standard curves were utilized to quantify gene expression in the samples. A Biorad CFX96 Real-Time 
PCR Detection System was used for amplification using the following protocol: denaturation at $95^{\circ} \mathrm{C}$ for 10 minutes, followed by 50 cycles of amplification $\left(15\right.$ seconds $95^{\circ} \mathrm{C}$ and 1 minute $60^{\circ} \mathrm{C}$ ) followed by a dissociation curve. Data were analyzed using Biorad CFX Manager Software version 3.1, based on the relative quantification of mRNA expression of the target gene normalized to a housekeeping gene (ATDC5/MCT: $\beta$-actin, human fibroblasts: GAPDH). Primer sequences are depicted in Table 2.

Table 2. RT-qPCR primer sequences

\begin{tabular}{|c|c|c|}
\hline Gene & Forward & Reverse \\
\hline$\beta$-Actin & CCGAGCGCGAGATCGT & TGGCCATCTCGTTCTCGAA \\
\hline Aggrecan & CATGAGAGAGGCGAATGGAA & TGATCTCGTAGCGATCTTTCTTCT \\
\hline Alpl (Mm) & CCGATGGCACACCTGCTT & GGAGGCATACGCCATCACAT \\
\hline$A L P L(H s)$ & CCGTGGCAACTCTATCTTTGG & CAGGCCCATTGCCATACAG \\
\hline Bapx1 & ACCTGGCAGCTTCGCTGAA & AGGTCGGCGGCCATCT \\
\hline Col2a1 $(\mathrm{Mm})$ & TGGGTGTTCTATTTATTTATTGTCTTCCT & GCGTTGGACTCACACCAGTTAGT \\
\hline COL2A1 $(H s)$ & TGGGTGTTCTATTTATTTATTGTCTTCCT & GCGTTGGACTCACACCAGTTAGT \\
\hline Col10a1 $(\mathrm{Mm})$ & CATGCCTGATGGCTTCATAAA & AAGCAGACACGGGCATACCT \\
\hline COL10A1 (Hs) & ATGATGAATACACCAAAGGCTACCT & ACGCACACCTGGTCATTTTCTG \\
\hline Clb2 & TGCCAAGCTTTCTCTGATGCT & GGGTTCTCCCTGTCCTCGTT \\
\hline GAPDH $(H s)$ & ACTTTGTGAAGCTCATTTCCTGGTA & GTGGTTTGAGGGCTCTTACTCCTT \\
\hline ITS1 (Mm) & TGGGGGGGTGGATGTCTGGAG & CGAGTGATCCACCGCTAAGAGTCGTA \\
\hline ITS1 (Hs) & TGTGAAACCTTCCGACCCCTCT & CGAGTGATCCACCGCTAAGAGTCGTA \\
\hline Mef2c & GGGCCTCAATGGCTGTGA & CTCAGACTCAGGGCTGTGACCTA \\
\hline Osteocalcin & GCGGCCCTGAGTCTGACA & GCCGGAGTCTGTTCACTACCTT \\
\hline Pop4 & GGACACGCAGCCACAGATG & TGACTGAAATAATAGCACCATGAAGAT \\
\hline Pthrp (Mm) & GTTCAGCAGTGGAGTGTC & GATGGTGGAGGAAGAAACG \\
\hline PTHrP (Hs) & AAGGGCAAGTCCATCCAAGA & CTCGGCGGTGTGTGGATTTC \\
\hline $\operatorname{Rmrp}(M m)$ & ATACGAGGGACATGTTCCTTATCC & TTGGCGGGCTAACAGTGACT \\
\hline$R M R P(H s)$ & GAGAGTGCCACGTGCATACG & ACGCTTCTTGGCGGACTTT \\
\hline Rpp30 & TCAAAAGACCCCCTGTTAATGTG & GATAGCAGGACCATAGACAAGTTCAA \\
\hline Rpp40 & TGTGTCACTACTTCGATGAACCAA & TGTCTGCAAAGCCTTGAACTGT \\
\hline Runx2 & GACGAGGCAAGAGTTTCACC & GGACCGTCCACTGTCACTTT \\
\hline $5.8 \mathrm{~S}$ rRNA & CACTCGGCTCGTGCGTCGAT & CGCTCAGACAGGCGTAGCCC \\
\hline
\end{tabular}




\begin{tabular}{|l|l|l|}
\hline Gene & Forward & Reverse \\
\hline 18S rRNA & AGTCCCTGCCCTTTGTACACA & GATCCGAGGGCCTCACTAAAC \\
\hline 28S rRNA & GCCATGGTAATCCTGCTCAGTAC & GCTCCTCAGCCAAGCACATAC \\
\hline Sox9 & AGTACCCGCACCTGCACAAC & TACTTGTAGTCCGGGTGGTCTTTC \\
\hline Viperin & TGCTATCTCCTGCGACAGCTT & CCTTGACCACGGCCAATC \\
\hline
\end{tabular}

When not specified, primer sequences were designed for Mus musculus. In table Mus musculus is abbreviated as $\mathrm{Mm}$ and Homo sapiens as $\mathrm{Hs}$.

\section{Fixated cell staining protocols and assays}

\section{Crystal Violet, Alcian Blue and Alizarin Red S staining and quantification}

Cells were washed two times with $0.9 \% \mathrm{NaCl}$ and fixated with $4 \%$ paraformaldehyde in PBS for 10 minutes at room temperature. Fixated cells were washed 6 times with distilled water and air-dried. Dried fixated cells were incubated for 30 minutes at room temperature with either $0.1 \%$ (m/v) Crystal Violet (Sigma-Aldrich); 1\% (m/v) Alcian Blue (Acros Organics, Geel, Belgium) in $0.1 \mathrm{M} \mathrm{HCl}$; or $40 \mathrm{mM}$ Alizarin Red S (Sigma-Aldrich), pH 4.2. Cells were washed six times with distilled water to remove unbound stain and allowed to air dry. Pictures were acquired with an Epson V370 flatbed scanner (Epson, Nagano, Japan). Crystal Violet was extracted by incubation with $10 \%$ acetic acid (VWR) for 15 minutes on a plate shaker (IKA HS 260 Basic, IKA, Staufen, Germany). Alcian Blue was extracted by incubation with $6 \mathrm{M}$ Guanidine-HCl (Sigma-Aldrich) for 2 hours on a plate shaker (IKA). Alizarin Red S extraction was initiated by incubation with $10 \%$ acetic acid (VWR Prolabo) for 30 minutes at room temperature on a plate shaker (IKA). Cells and eluate were further collected using a cell scraper and transferred to a microcentrifuge tube. Samples were heated at $85^{\circ} \mathrm{C}$ for 10 minutes and subsequently centrifuged at $20,000 \times \mathrm{g}$ for 15 minutes at $4^{\circ} \mathrm{C}$. Ammonium hydroxide (VWR) was added to neutralize the acetic acid until a $\mathrm{pH}$ of 4.4 was reached. Extracted Crystal Violet, Alcian Blue and Alizarin Red $S$ were quantified spectrophotometrically at $590 \mathrm{~nm}, 645 \mathrm{~nm}$ or $405 \mathrm{~nm}$ (respectively) using a plate reader (ThermoScientific Multiskan FC, Waltham, MA, USA). 


\section{ALP activity assay}

Enzymatic activity of Alkaline Phosphatase in ATDC5 cultures was determined in a colorimetric assay. Cells were lysed in lysis buffer (1.5 M Tris- $\mathrm{HCl}, \mathrm{pH} 9.0 ; 2 \%$ v/v Triton X100) and homogenized on ice by means of sonication (MSE Soniprep 150, Gemini, Apeldoorn, the Netherlands). Insoluble material was removed by centrifugation (5 minutes, 13,000 at $4^{\circ} \mathrm{C}$ ). Total protein concentration was determined with a BCA assay (SigmaAldrich). In flat-bottom 96 wells plates containing assay buffer $(1.5 \mathrm{M}$ Tris- $\mathrm{HCl}, \mathrm{pH}$ 9.0, $1 \mathrm{mM}$ $\mathrm{MgCl}_{2} ; 7.5 \mathrm{mM}$ p-nitrophenyl phosphate), ALP activity was determined by measuring ALPdependent enzymatic conversion of p-nitrophenyl phosphate to nitrophenyl phosphate by spectrophotometric analysis at $405 \mathrm{nM}$. A calibration curve containing an increasing concentration of nitrophenyl phosphate was used to determine the absolute amount of ALP-generated nitrophenyl phosphate over time. Values were normalized to total protein concentration and ALP activity expressed as $\mu \mathrm{mol}$ nitrophenyl phosphate / gram / minute.

\section{Chondrogenic trans-differentiation of human dermal fibroblasts}

Human dermal fibroblasts (passage 7-9) from $4 \mathrm{CHH}$ patients and 3 healthy controls (ethical permission was obtained from the medical ethical Institutional Review Board of Freiburg University Hospital and methods and experimental protocols to obtain dermal fibroblasts were carried out in accordance with the Freiburg University Hospital medical ethical Institutional Review Board, according to German law. Informed consent was obtained from all subjects) were hyperconfluently plated $\left(100,000\right.$ cells $\left./ \mathrm{cm}^{2}\right)$ in Aggrecan-coated (SigmaAldrich; $2.5 \mu \mathrm{g} / \mathrm{cm}^{2}$ ) wells as previously described ${ }^{34}$. Cells were directly plated in transdifferentiation medium which consisted of DMEM/F12+Glutamax (Invitrogen), 10\% FCS (PAA), 1\% antibiotic/antimycotic (Invitrogen), 1\% NEAA (Invitrogen) + 1\% ITS (Insulin, Transferrin, Selenium-Sodium Pyruvate; Life Technologies), $50 \mu \mathrm{g} / \mathrm{ml}$ ascorbic acid 2phosphate (Sigma-Aldrich) and $1 \mathrm{ng} / \mathrm{ml}$ human recombinant TGF- $\beta 3$ (Life Technologies). Trans-differentiation medium was refreshed every other day and transdifferentiated cartilaginous nodules were harvested in TRIzol on day 3, 5 and 7 during transdifferentiation. At plating, fibroblasts were harvested for day 0 samples as well. 


\section{Results}

\section{Rmrp RNA and RNase MRP protein subunits are differentially expressed in the developing growth plate}

To investigate RMRP RNA expression during chondrogenic differentiation of the growth plate, $5 \mu \mathrm{m}$ tissue sections were prepared from growth plates of 6 weeks-old mice. Expression of Rmrp RNA was determined by in situ hybridization (Figure 1A). Resting zone chondrocytes express Rmrp RNA and weak expression was observed in the chondrocytes of the proliferative zone. The highest expression levels of Rmrp RNA were detected in the hypertrophic zone. Cells in the remodeling zone of the growth plate (osteoclasts and osteoblasts) were also positive for the expression of Rmrp RNA. Subsequently, we evaluated the spatiotemporal expression of a number of RNase MRP protein subunits RPP25, RPP30, RPP38, RPP40 and POP1 (Figure 1B). Expression of COL10A1 was immunohistochemically detected to visualize the location of the hypertrophic zone (Figure 1B). Without exception, all RNase MRP protein subunits tested displayed a spatiotemporal growth plate distribution pattern highly similar to Rmrp RNA; resting zone chondrocytes express RNase MRP subunits, whereas weak expression was observed in chondrocytes of the proliferative zone. Highest RNase MRP protein subunit expression levels were detected in the hypertrophic zone of the developing growth plate. 


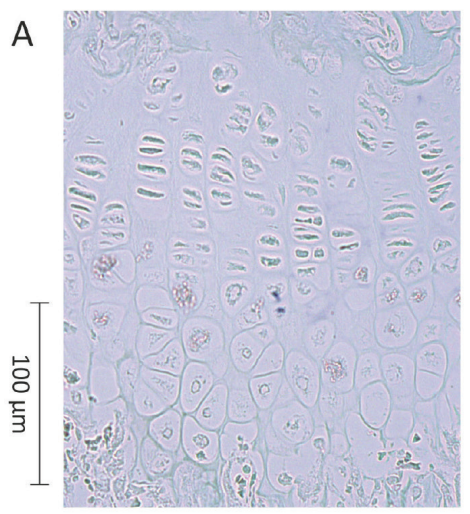

Scrambled

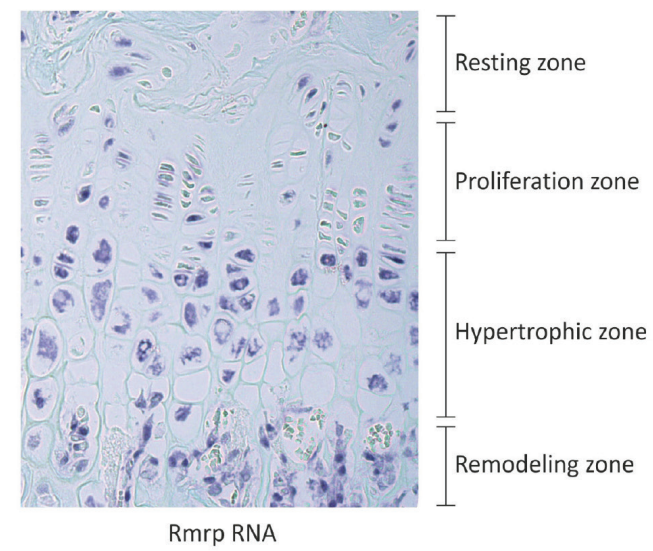

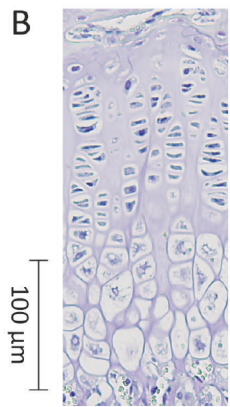

Control

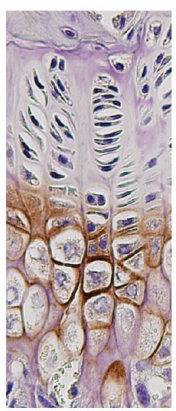

COL10A1

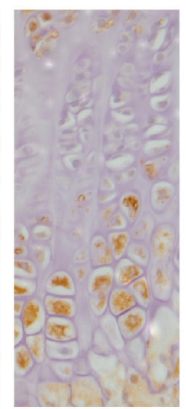

RPP25

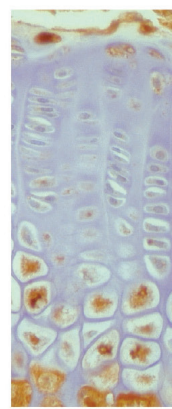

RPP30

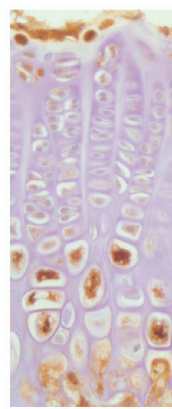

RPP38

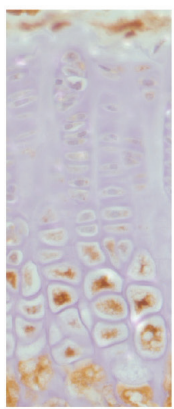

RPP40

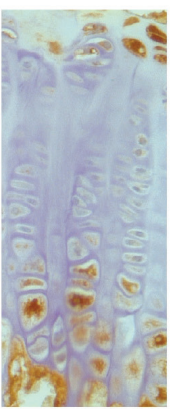

POP1

Figure 1: Spatiotemporal expression of Rmrp RNA and RNase MRP subunits in the growth plate. Five micrometer-thick formalin-fixed paraffin-embedded tissue sections were prepared from knee joint epiphyseal growth plates of 6-weeks-old mice. A: Expression of Rmrp RNA was detected by in situ hybridization. A scrambled probe was used as control. B: The expression of COL10A1 and RNase MRP protein subunits RPP25, RPP30, RPP38, RPP40 and POP1 was detected immunohistochemically. Figure shows representative images of the ISH (A) IHC (B) results from three individual experiments each.

\section{Rmrp RNA expression in induced during ATDC5 chondrogenic differentiation}

To study RMRP RNA expression in a model for chondrogenic differentiation we used the ATDC5 cell line ${ }^{31}$. ATDC5 cells follow a defined chondrogenic differentiation program after stimulation with insulin, transferrin and sodium selenite. The induction of Col2a1 expression 
at day 7 during differentiation marks chondrogenic differentiation. At 14-21 days during differentiation cells have acquired a predominant hypertrophic/mineralizing phenotype as indicated by co-expression of Col10a1, and at day 21 mineralization becomes evident by expression of osteocalcin (Figure 2A). Expression of Rmrp RNA was slightly upregulated at day 7 in chondrogenic differentiation, increased further at day 14 in differentiation and showed highest levels at day 14 and 21 in differentiation, predominantly coinciding with peak Col10a1 expression. Rmrp RNA expression decreased again at day 28 during differentiation (Figure 2B). Expression of RNase MRP protein subunits Rpp30 and Rpp40 showed a similar pattern with the highest levels of expression at day 14 and 21 during differentiation (Figure 2B). Together, these data show that the expression of Rmrp RNA responds to induction of chondrogenic differentiation with highest expression levels late in the differentiation program.

A

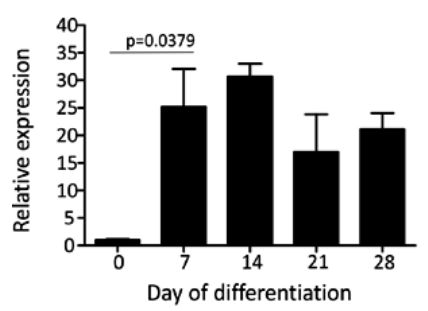

B

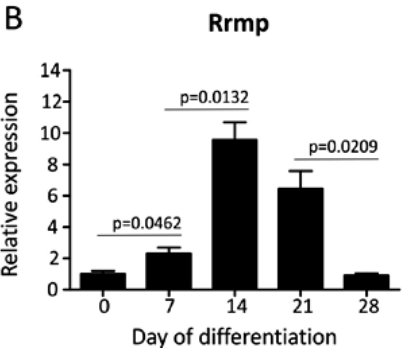

Col10a1

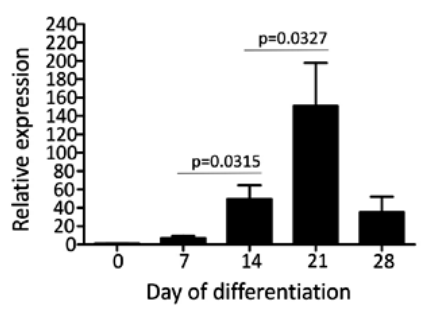

Rpp30

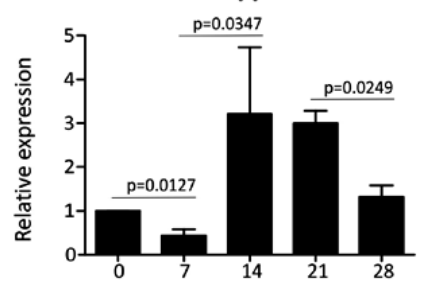

Day of differentiation
Osteocalcin

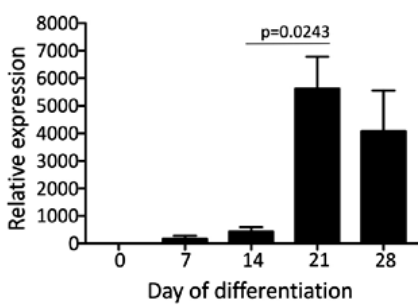

Rpp40

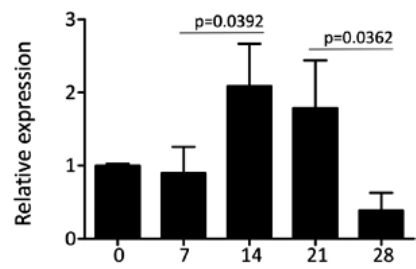

Day of differentiation

Figure 2: Rmrp RNA and RNase MRP subunit expression responds to chondrogenic differentiation of ATDC5 cells. ATDC5 cells were differentiated in the chondrogenic lineage for 0, 7, 14, 21 or 28 days. A: Different stages of chondrogenic differentiation were confirmed by measuring gene expression of Col2a1, Col10a1 and osteocalcin. B: Expression of Rmrp RNA and RNase MRP protein subunits $R p p 30$ and $R p p 40$ is depicted as fold induction relative to $\mathrm{t}=0$. Data was normalized to 6 -actin and represents the average value of 3 biological replicates plus standard deviation. For statistical evaluation an independent samples t-test was performed between each 
consecutive time point using GraphPad Prism 5. p-values are indicated. Presented graphs are representative examples of three independent experiments.

\section{Rmrp expression is positively correlated with the hypertrophic phenotype of the chondrocyte}

Above data indicate that RMRP RNA expression is associated with late phase/hypertrophic chondrogenic differentiation. Therefore we next asked whether RMRP RNA expression adapts to a changing chondrocyte hypertrophic phenotype. To drive differentiating ATDC5 cells towards a hypertrophic phenotype we reduced the expression of Sox9 or Bapx1 by targeting the corresponding mRNAs by siRNA-mediated knockdown. In line with previous reports and work from our group ${ }^{33,35-37}$, knockdown of Sox9 expression (Figure 3A) resulted in reduced expression of chondrogenic markers Bapx1 (Figure 3B), Col2a1 (Figure 3C) and Aggrecan (Figure 3D) and concomitantly increased expression of hypertrophic markers Runx2 (Figure 3E), Mef2c (Figure 3F) and Col10a1 (Figure 3G), which is consistent with a prominent hypertrophic chondrocyte phenotype. As a result of Sox9 knockdown, the expression of Rmrp RNA (Figure $3 \mathrm{H}$ ) was increased. We previously reported ${ }^{35}$ that knockdown of the key hypertrophic repressor Bapx1 (Figure 3B) does not influence the expression of chondrogenic factors Sox9, Col2a1 and Aggrecan (Figure 3A, C-D). Instead, a selective hypertrophic chondrocyte phenotype was provoked, as evidenced by increased expression of hypertrophic markers Runx2, Mef2c and Col10a1 (Figure 3E-G). Similar to what was observed for Sox9 knockdown, preferential hypertrophic differentiation by knockdown of Bapx1 expression led to increased expression of Rmrp RNA (Figure 3H). Increased hypertrophy was also observed when ATDC5 cells were exposed to the hypertrophic inducer BMP- ${ }^{36}$, as evidenced by induced expression of hypertrophic markers. Under these conditions, the expression of Rmrp RNA was also induced (Figure $3 \mathrm{H}$ ). 

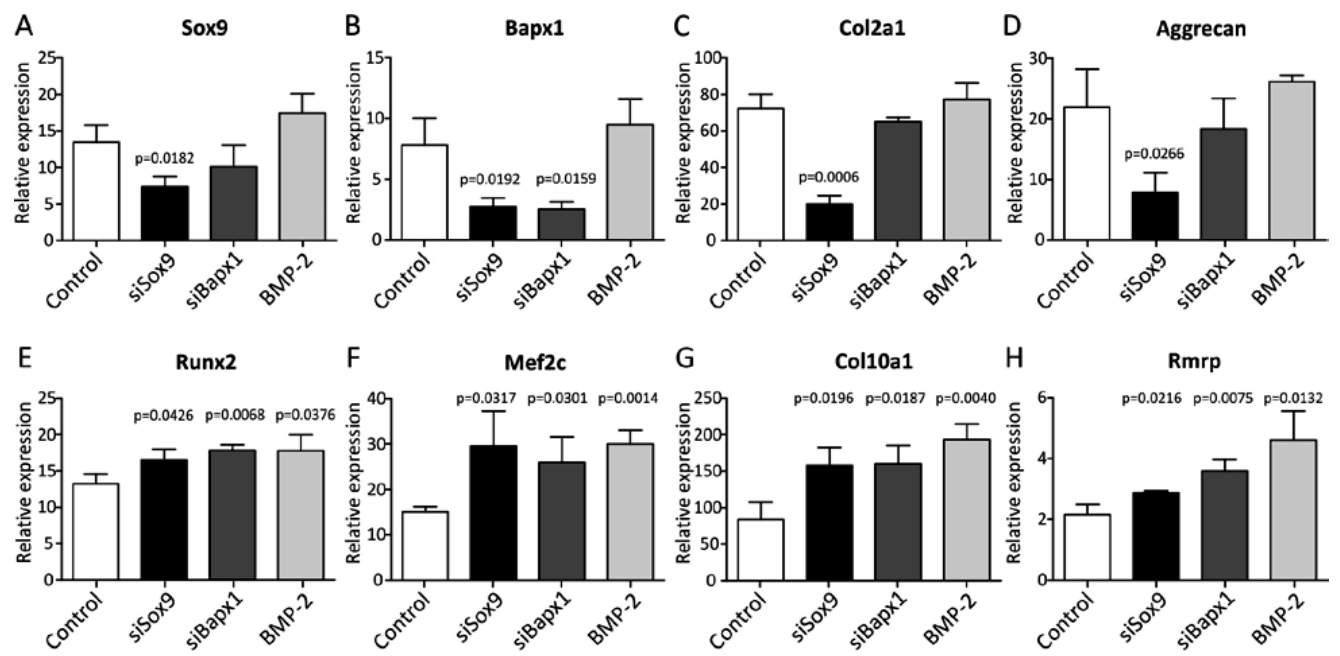

Figure 3: Rmrp RNA expression increases with ATDC5 hypertrophic differentiation. ATDC5 cells were transfected with 100 nM siRNA duplexes targeting Sox9 or Bapx1 gene expression on day -1 , day 2 , and day 5 during chondrogenic differentiation. A scrambled siRNA duplex purchased from Eurogentec was used as control condition for both Sox9 or Bapx1 knockdown. In addition, ATDC5 cells transfected with the scrambled siRNA were exposed to $30 \mathrm{ng} / \mathrm{ml}$ BMP-2 from the start of differentiation (cells from the BMP-2 condition were also transfected with the negative control scrambled siRNA in order to make all three interventions (Sox9 knockdown, Bapx1 knockdown and exposure to BMP-2) technically comparable to the same control). RNA was isolated from these cultures at day 10 in differentiation. Gene expression of Sox9 (A), Bapx1 (B), Col2a1 (C), Aggrecan (D), Runx2 (E), Mef2c (F), Col10a1 (G) and Rmrp RNA (H) was determined by RT-qPCR. Gene expression is depicted as fold induction relative to $\mathrm{t}=0$. Data was normalized to $B$-actin expression and represents the average values of 3 biological replicates plus standard deviation. For statistical evaluation an independent samples t-test was performed relative to control using Graphpad Prism 5. p-values are indicated. Experiment was performed three times with presented graphs being representative examples.

To further investigate Rmrp induction by chondrocyte hypertrophy, ATDC5 cell were differentiated for 14 days to induce a hypertrophic phenotype. To counteract hypertrophy, cultures were exposed to PTHrP from day 10 in differentiation onwards ${ }^{23,38}$. Addition of PTHrP reduced the induction of the hypertrophic markers Runx2 (Figure 4A) and Col10a1 (Figure 4B). As a result of PTHrP-mediated reduction of hypertrophy, Rmrp RNA expression 
was downregulated as well (Figure 4C). To confirm the PTHrP-mediated reduction of Rmrp RNA expression in another hypertrophic chondrocyte model, we used MCT cells. MCT cells are mouse chondrocytes, immortalized with a temperature-sensitive simian virus 40 large tumor antigen. These cells proliferate at $32^{\circ} \mathrm{C}$, but terminally differentiate and become hypertrophic within 24 hours at $37^{\circ} \mathrm{C}^{32}$. As expected, culturing $\mathrm{MCT}$ cells at $37^{\circ} \mathrm{C}$ induced hypertrophic differentiation as indicated by upregulation of Runx2 and Col10a1 expression (Figure 4D/E). Rmrp RNA expression was also upregulated in this hypertrophic cell model (Figure 4F). When the hypertrophic MCT phenotype was suppressed by PTHrP (Figure 4D/E), Rmrp RNA expression responded similarly (Figure 4F). Overall, our data indicate a positive correlation between Rmrp RNA expression levels and chondrocyte hypertrophy.
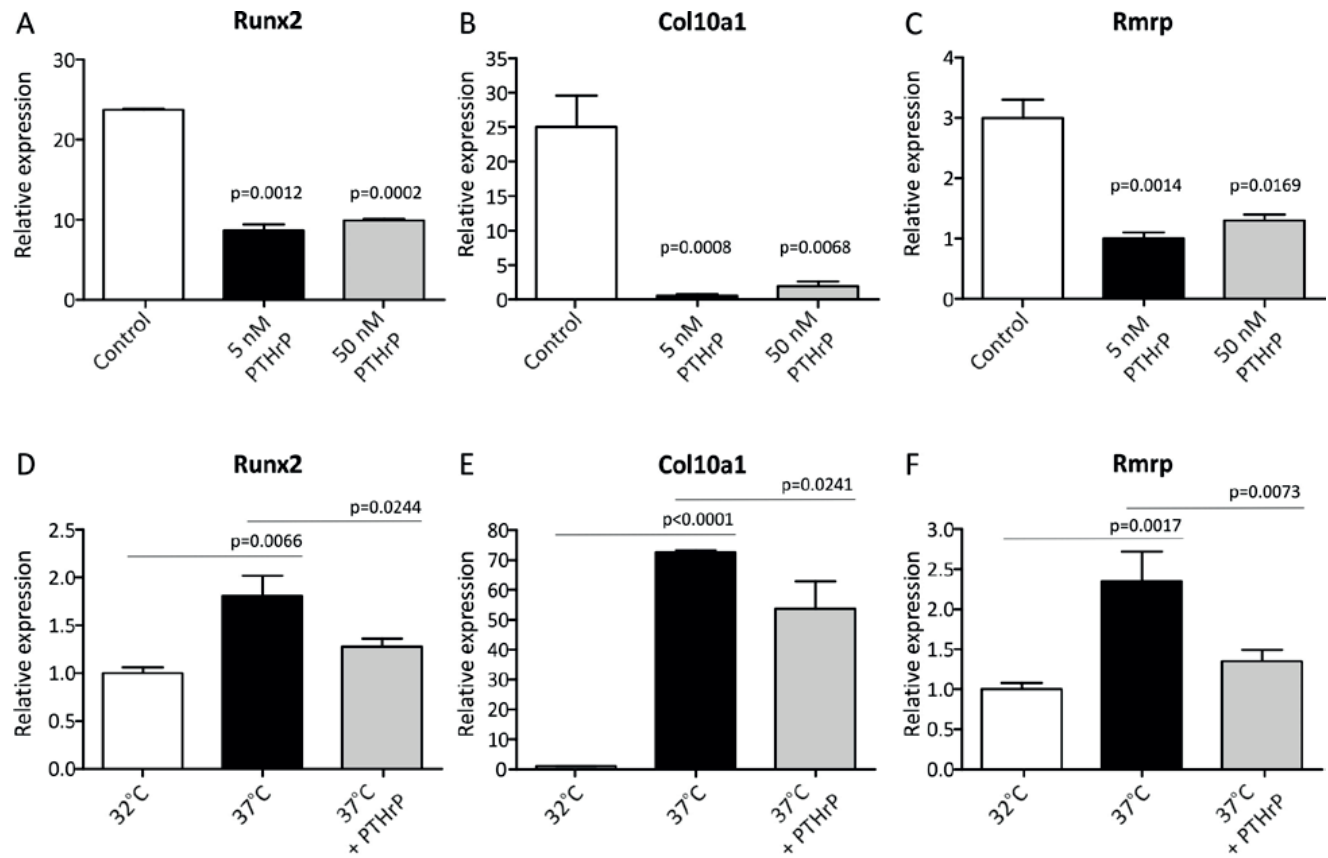

Figure 4: Hypertrophy-associated Rmrp RNA expression is reduced by PTHrP. ATDC5 cells were differentiated for 14 days to create a hypertrophic phenotype and 5 or $50 \mathrm{nM}$ PTHrP was added from day 10 in chondrogenic differentiation onwards. Gene expression of Runx2 (A) and Col10a1 (B) and Rmrp RNA (C) was analyzed by RT-qPCR. Data was normalized to 8 -actin, represents the average of value of 3 individual samples plus standard deviation and is depicted as fold induction relative to day 0 ATDC5 cells. For statistical evaluation an independent samples t-test was performed 


\section{Chapter 2}

relative to day 14 control ATDC5 cells using Graphpad Prism, where ${ }^{*}=p<0.05$. MCT cells were plated and cultured at the permissive temperature of $32^{\circ} \mathrm{C}$ and hypertrophic differentiation was induced by incubating the cells at $37^{\circ} \mathrm{C}$ in the absence or presence of $50 \mathrm{nM}$ PTHrP. Total RNA was isolated 24 hours after the temperature shift and analyzed for Runx2 (D), Col10a1 (E) and Rmrp RNA (F) expression by RT-qPCR. Data was normalized to 8 -actin, represents the average value of 4 biological replicates plus standard deviation and is depicted as fold-induction relative to the permissive temperature of $32^{\circ} \mathrm{C}$. For statistical evaluation an independent samples t-test was performed between the permissive temperature and induction of hypertrophic differentiation and between hypertrophic induction in the absence or presence of PTHrP using Graphpad Prism 5. p-values are indicated. Presented graphs are representative examples of three independent experiments.

\section{Chondrogenic morphogens alter Rmrp promoter activity}

Our data described above suggested that RMRP RNA expression may be responsive to common chondrogenic morphogens, for example via modulation of proximal promoter activity. To acquire a broader understanding of chondrogenic pathways that may influence RMRP RNA expression, ATDC5 cells were transfected with a Gaussia luciferase reporter, driven by the 1500 nucleotide sequence upstream of the mouse Rmrp transcription start site (pGluc-Rmrp-prom plasmid) and cells were exposed to different chondrogenic mediators (Figure 5). PTHrP keeps chondrocytes in a proliferative state and counteracts hypertrophic differentiation ${ }^{23,38}$. PTHrP decreased Rmrp promoter activity by $18 \%$. bFGF (or FGF2), a negative regulator of chondrocyte hypertrophic maturation ${ }^{39}$, reduced $R m r p$ promoter activity by $35 \%$. TGF $\beta$ isoforms activate SMAD signaling via SMAD $2 / 3^{40}$. Of all TGF $\beta$ isoforms, TGF $\beta 3$ was described to be able to induce matrix mineralization ${ }^{41}$. Indeed, TGFB3 increased Rmrp promoter activity by $40 \%$. In agreement with the TGF 33 -induced increase of Rmrp promoter activity, exposure to (5Z)-7-Oxozeaenol, an inhibitor of TGF- $\beta$ activated kinase- 1 (TAK1) ${ }^{42}$, decreased Rmrp promoter activity by $22 \%$. Exposure to BMP-2 (pro-hypertrophic, see Figure 3) increased Rmrp promoter activity by $105 \%$, whereas dorsomorphin, an inhibitor of BMP-mediated SMAD1/5/8 phosphorylation ${ }^{43}$, decreased Rmrp promoter activity by $76 \%$. GDF-5 (a BMP family member described to induce both chondrogenic differentiation and hypertrophy ${ }^{44}$ did not significantly change $R m r p$ promoter activity. WNT-3A and WNT-5A, two prominent ligands of the Wnt signaling pathway and crucial in chondrocyte differentiation ${ }^{45}$, increased $R m r p$ promoter activity by $45 \%$ and $26 \%$, 
respectively. Finally, Sonic Hedge $\mathrm{Hog}(\mathrm{SHH})$, the main ligand that activates Patched-1mediated GLI-signaling and which is crucial in limb bud patterning during embryogenesis ${ }^{46}$, did not significantly alter promoter activity. We thus concluded that the 1500 nucleotide sequence upstream of the Rmrp RNA transcription start site is responsive to mediators known to alter the chondrocyte phenotype. This indicates that, in chondrocytic cells, Rmrp RNA levels may be controlled by transcription factors involved in chondrocyte differentiation and hypertrophy.

\section{Rmrp promoter activity}

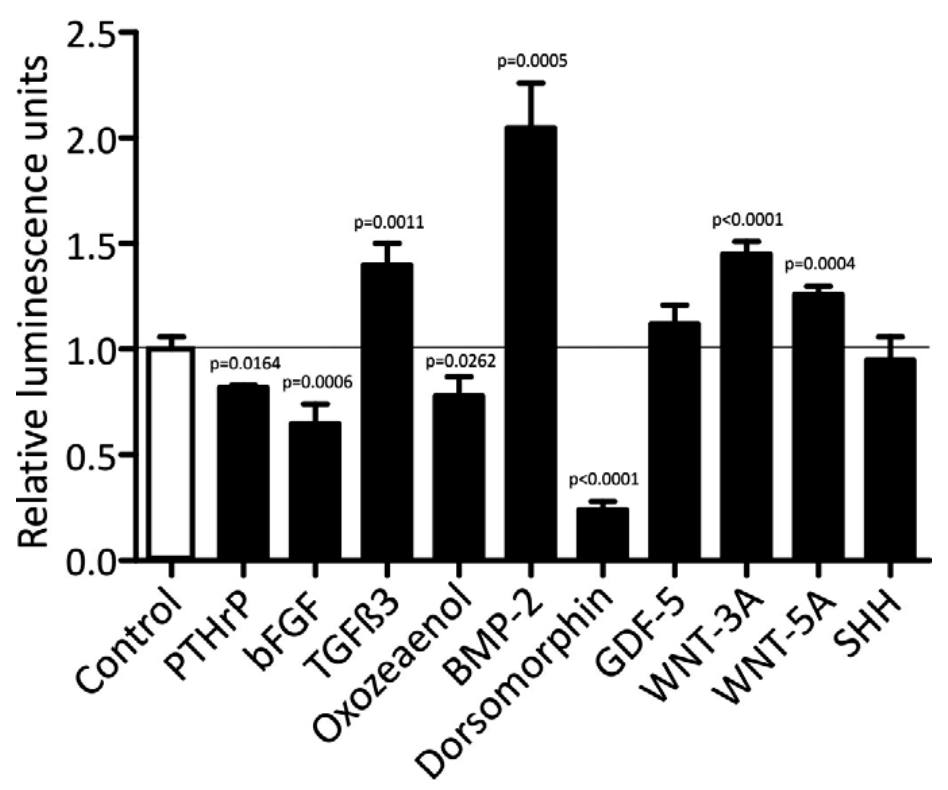

Figure 5: Rmrp promoter activity is altered by chondrogenic morphogens. Rmrp promoter responsiveness to different chondrogenic morphogens and pathways was determined by transfection of the pGluc-Rmrp-prom reporter plasmid, comprising the 1500 nucleotide sequence upstream of the mouse Rmrp RNA transcription start site. ATDC5 cells were transiently cotransfected with this reporter and pGL4.20[luc2/Puro]-CMV to normalize for transfection efficiency. Transfected cells were exposed for 24 hours to either $100 \mathrm{nM}$ PTHrP, $15 \mathrm{ng} / \mathrm{ml} \mathrm{bFGF,} 10 \mathrm{ng} / \mathrm{ml}$ TGFß3, $0.5 \mu \mathrm{M}$ (5Z)-7-Oxozeaenol, $30 \mathrm{ng} / \mathrm{ml}$ BMP-2, $10 \mu \mathrm{M}$ dorsomorphin, $100 \mathrm{ng} / \mathrm{ml}$ GDF-5, 15 $\mathrm{ng} / \mathrm{ml}$ WNT-3A, $15 \mathrm{ng} / \mathrm{ml}$ WNT-5A or $150 \mathrm{ng} / \mathrm{ml} \mathrm{SHH}$. These concentrations were chosen based on their use as described in literature. Promoter activity is depicted as fold induction relative to control. 
Data was normalized to pGL4.20[luc2/Puro]-CMV and represents the average value of 4 biological replicates plus standard deviation. For statistical evaluation an independent samples t-test was performed relative to control using GraphPad Prism 5. p-values are indicated. Presented graphs are representative examples of three independent experiments.

\section{Rmrp RNA knockdown deregulates chondrogenic differentiation of ATDC5 cells}

To investigate whether RMRP RNA regulates chondrogenic differentiation, Rmrp RNA expression was targeted by RNAi. ATDC5 cells were transfected with an siRNA duplex at day $-1,2$ and 5 during chondrogenic differentiation and gene expression was determined at day 0,7 and 10 in differentiation. The siRNA duplex reduced the expression of Rmrp RNA significantly (Figure 6A). To confirm that this led to a functional reduction of RNase MRP activity, the expression of substrate RNAs for RNase MRP, i.e. Clb2 mRNA, Viperin mRNA and ITS1 pre-rRNA, were measured ${ }^{4-6}$. Reduced Rmrp RNA levels indeed resulted in elevated levels of RNase MRP substrates Clb2 and Viperin mRNAs, as well as an accumulation of an ITS1 pre-rRNA processing intermediate. (Figure 6B-D). Following Rmrp knock-down, expression of Sox9 (Figure 6E), Col2a1 (Figure 6F), Runx2 (Figure 6G), Col10a1 (Figure 6H) and $A / p l$ (Figure $6 \mathrm{I}$ ) was reduced at both 7 and 10 days in differentiation. Overall, expression of Runx2, Col10a1 and Alp/ seemed to be more heavily affected than Col2a1. In accordance, Bapx1 mRNA was strongly induced (Figure 6J), which is indicative of deregulated hypertrophic differentiation ${ }^{35}$. These data show that reduction of Rmrp RNA levels impacts the course of chondrogenic differentiation, with a prominent impact on the hypertrophic differentiation program. In concordance with the observed deregulated chondrogenic differentiation, expression of Pthrp was increased after Rmrp RNA knockdown (Figure 6K). Since RNase MRP has been shown to be implicated in pre-rRNA processing ${ }^{3,4}$, we assessed the levels of $18 \mathrm{~S}, 5.8 \mathrm{~S}$ and $28 \mathrm{~S}$ following Rmrp RNA knockdown and we observed reduced levels of $18 \mathrm{~S}$ and $5.8 \mathrm{~S}$, but not of $28 \mathrm{~S}$ rRNAs at day 7 in ATDC5 chondrogenic differentiation (Figure 6L). 

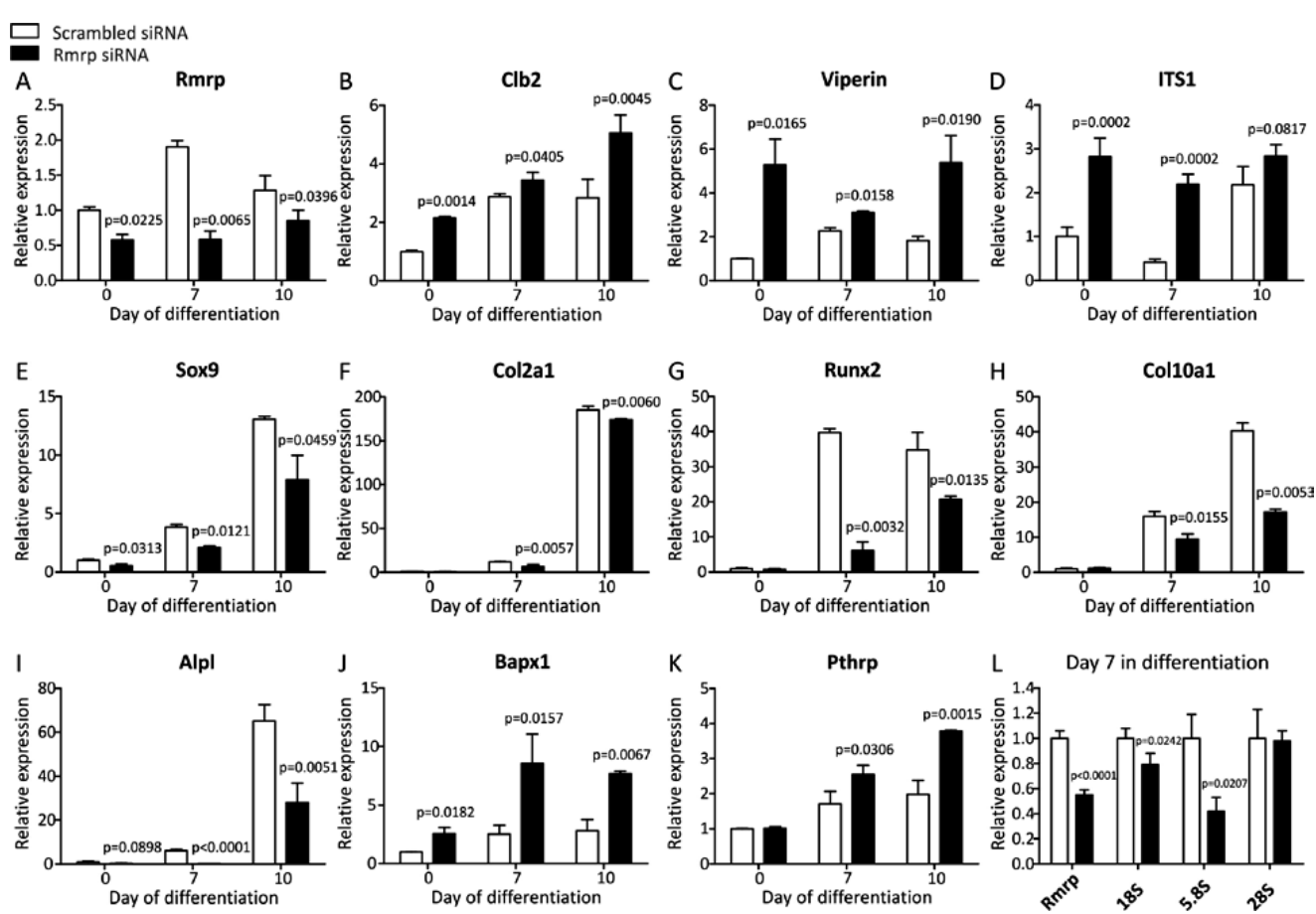

Figure 6: Rmrp RNA knockdown deregulates ATDC5 chondrogenic differentiation. ATDC5 cells were transfected with 100 nM siRNA duplex targeting Rmrp RNA expression on day -1, day 2 , and day 5 during chondrogenic differentiation. A scrambled siRNA was used as control condition. RNA was isolated from these cultures at day 7 and 10 in differentiation and at $t=0$ as a reference. Expression of Rmrp (A), Clb2 (B), Viperin (C), ITS1 rRNA processing intermediate (D), Sox9 (E), Col2a1 (F), Runx2 (G), Col10a1 (H), Alpl (I), Bapx1 (J), PthrP (K) and Rmrp, 18S, 5.8S and 28S (L) was determined by means of RT-qPCR. Gene expression is depicted as fold induction relative to $t=0$ (A-K). In Figure $6 \mathrm{~L}$ day 7 scrambled controls are set to 1 . Data was normalized to 6 -actin and represents the average value of 3 (Figure $6 \mathrm{~A}-\mathrm{K}$ ) or 4 (Figure 6L) biological replicates plus standard deviation. For statistical evaluation an independent samples t-test was performed relative to scrambled control for each consecutive time point using GraphPad Prism 5. p-values are indicated. Presented graphs are representative examples of three independent experiments. 


\section{Rmrp RNA knockdown affects cell proliferation, glycosaminoglycan content and mineralization of ATDC5 cells}

We next investigated whether deregulation of chondrogenic differentiation by Rmrp RNA knockdown is accompanied by functional changes of the chondrogenic differentiation program at different moments in differentiation. To maintain Rmrp knockdown during an extended period of time during chondrogenic differentiation, siRNAs were either transfected on day 2 and 5 in chondrogenic differentiation for read-out at day 7 or on day 2 , 5 and 8 in chondrogenic differentiation for read-out at day 14. Rmrp RNA knockdown resulted in reduced Rmrp RNA expression at day 7 in chondrogenic differentiation (Figure 7A). Since day 14 cells were last transfected at day 8 in chondrogenic differentiation, $R m r p$ RNA expression was almost back to baseline levels in the day 14 Rmrp RNA knockdown condition (Figure 7A). Rmrp RNA knockdown resulted in reduced cell proliferation, quantified in Figure 7B and visually depicted in Figure 7C/D. Rmrp RNA knockdown led to a significant reduction of GAG content at day 7 and this was still detectable at day 14 in chondrogenic differentiation (Figure 7E). GAG content at day 14 before extraction of the Alcian Blue dye is visualized in Figure 7F. Mineralization, quantified by Alizarin Red staining was reduced at day 7 and day 14 in chondrogenic differentiation (Figure 7G) and visualized in Figure 7H/I. In concert with the Alizarin Red data, ALP enzyme activity was almost absent at day 7 in chondrogenic differentiation as a result of Rmrp knockdown and was still significantly reduced at day 14 in chondrogenic differentiation (Figure $7 \mathrm{~J}$ ). 

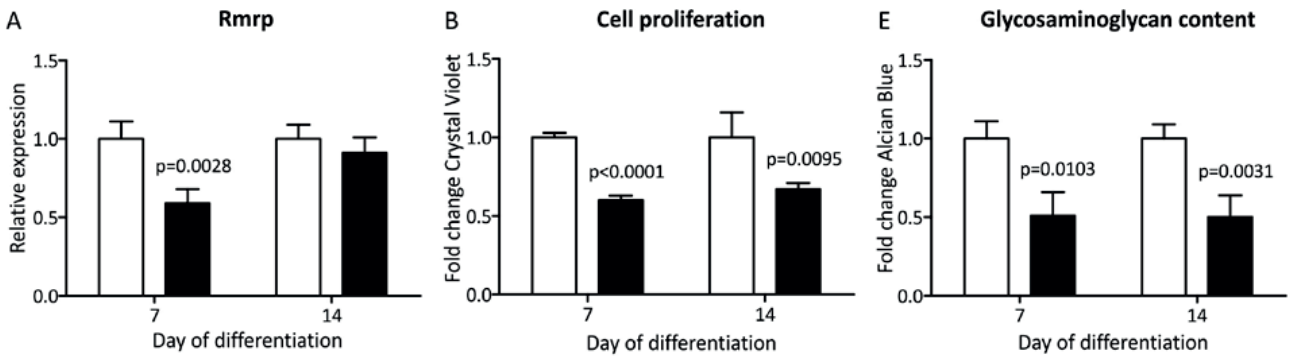

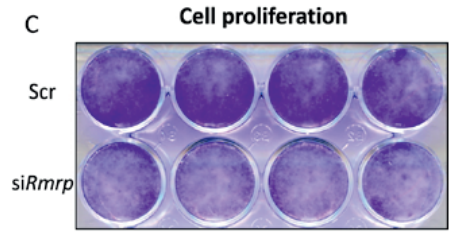

Day 7 in differentiation
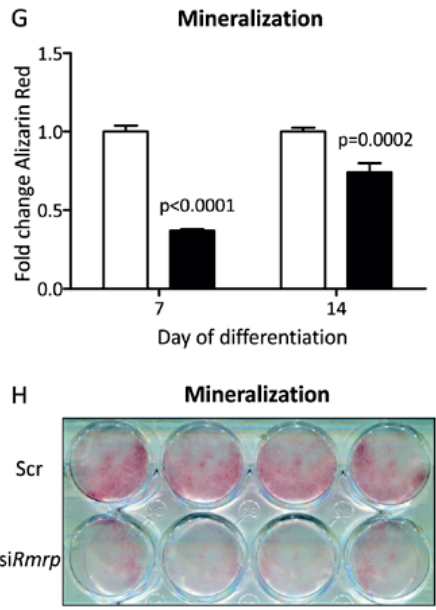

Day 7 in differentiation
D

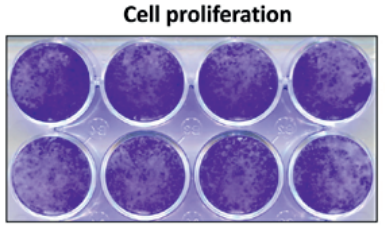

Day 14 in differentiation

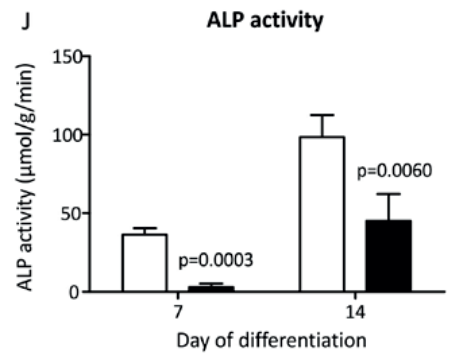

Mineralization

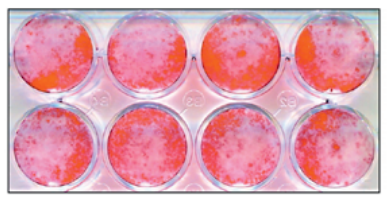

Day 14 in differentiation

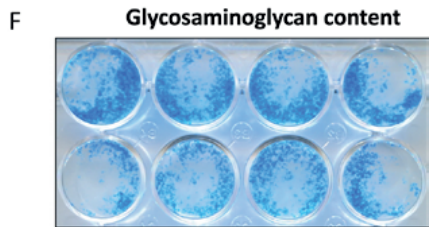

Day 14 in differentiation

Scrambled siRNA

Rmrp siRNA

Figure 7: Rmrp RNA knockdown reduces cell proliferation, glycosaminoglycan content and mineralization. ATDC5 cells were transfected with siRNA duplex targeting Rmrp RNA on day 2 and 5 in differentiation (30 and $100 \mathrm{nM}$, respectively) and analyzed on day 7. In addition differentiating ATDC5 cells were transfected with the Rmrp RNA siRNA at day 2, 5 and 8 (30 $\mathrm{nM}, 100 \mathrm{nM}$ and $100 \mathrm{nM}$, respectively) and analyzed at day 14 in differentiation. A scrambled siRNA was used as a control. Knockdown of Rmrp RNA expression was confirmed by RT-qPCR (A). Cell proliferation was determined by extraction and spectrophotometric analysis of bound Crystal Violet staining (B); staining before dye extraction is shown in (C) and (D). Glycosaminoglycan content was determined by extraction of Alcian Blue staining $(E)$; day 14 staining before dye extraction is shown in (F). Mineralization was determined by extraction of Alizarin Red staining (G); staining before dye 
extraction is visualized in (H) and (I). ALP activity in $\mu \mathrm{mol} / \mathrm{g} / \mathrm{min}$, corrected for total protein was determined in (J). Data (A, B, E, G, J) represents the average value of 4 biological replicates plus standard deviation and scrambled control is set to 1 (A, B, E, G). In graph (J) ALP activity in $\mu \mathrm{mol} / \mathrm{g} / \mathrm{min}$ was corrected for total protein levels. For statistical evaluation (A, B, E, G, J) an independent samples t-test was performed relative to scrambled control for each consecutive time point using GraphPad Prism 5. p-values are indicated. In (C, D, F, H, I) the upper panels show 4 biological replicates 'Scr' $=$ Scrambled siRNA and the lower panels show 4 biological replicates 'siRmrp' = Rmrp siRNA.

\section{Chondrogenic trans-differentiation is impaired in $\mathrm{CHH}$ fibroblasts}

$\mathrm{CHH}$ is caused by mutations in RMRP RNA ${ }^{14,17}$, but it is unknown whether these mutations influence chondrogenic differentiation in $\mathrm{CHH}$ patients. To test whether the chondrogenic capacity of $\mathrm{CHH}$ cells is impaired, we employed a trans-differentiation protocol that drives dermal fibroblasts towards a chondrocyte-like phenotype ${ }^{34}$. High density plating of fibroblasts on an Aggrecan-coated surface in the presence of TGF 33 induced the formation of dense aggregates within 24 hours post-plating, resembling chondrogenic nodules. To focus on functional chondrogenic read-out markers we measured induction of COL1OA1, COL2A1 and ALPL (alkaline phosphatase) gene expression in cultures from healthy fibroblasts, substantiating trans-differentiation into the chondrogenic lineage (Figure $8 \mathrm{~A}-\mathrm{C}$ ). Induction of COL1OA1 expression was more robust than COL2A1, which indicates that these cultures display a predominant hypertrophic phenotype (Figure 8A/B). As a result of the chondrogenic trans-differentiation RMRP RNA expression levels increased (Figure 8D). Compared to healthy control cultures we found that $\mathrm{CHH}$ fibroblasts $(4 \mathrm{CHH}$ patients, carrying different $\mathrm{CHH}$ pathogenic mutations in the RMRP gene (i.e. $127 \mathrm{G}>\mathrm{A}$ and $261 \mathrm{C}>\mathrm{G}$; 4 $\mathrm{C}>\mathrm{T}$ and $77 \mathrm{C}>\mathrm{T}$; $70 \mathrm{~A}>\mathrm{G}$ and 70A>G; $4 \mathrm{C}>\mathrm{T}$ and -21_-9dup CTCTGTGAAGCTG)) displayed an impaired induction of COL1OA1 and ALPL expression upon chondrogenic transdifferentiation (Figure $8 B / C$ ). Induction of COL2A1 expression was not affected at day 3 in trans-differentiation. At days 5 and 7 during chondrogenic trans-differentiation, COL2A1 expression appeared lower in $\mathrm{CHH}$ fibroblasts as compared to healthy controls (Figure 8A), although this difference was not significant. The induction of RMRP RNA expression observed in healthy control fibroblasts was absent in $\mathrm{CHH}$ cultures (Figure 8D). Moreover, 
and in concert with the observed impaired hypertrophic differentiation (Figure 8B/C), the expression of PTHrP was increased in $\mathrm{CHH}$ cultures as compared to healthy control cultures (Figure 8E). Finally, we detected a significant increase in the accumulation of the ITS1 prerRNA processing intermediate ${ }^{4}$ (Figure 8F). Together, data show that in $\mathrm{CHH}$ fibroblasts chondrogenic trans-differentiation is impaired with a major impact on hypertrophic development of these cultures.
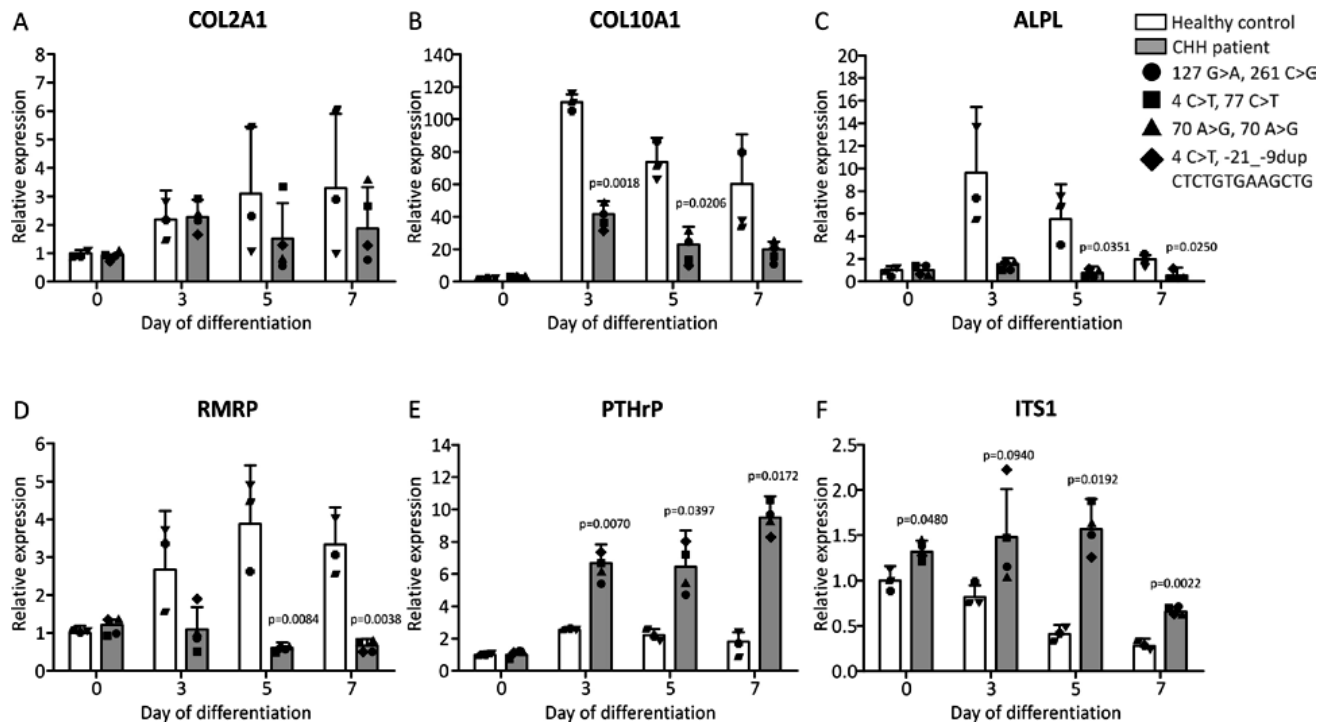

Figure 8: Impaired chondrogenic differentiation and increased ITS1 expression during chondrogenic trans-differentiation of $\mathrm{CHH}$ fibroblasts. Human dermal fibroblasts from four $\mathrm{CHH}$ patients (RMRP alleles of $\mathrm{CHH}$ patients carried the following mutations: $127 \mathrm{G}>\mathrm{A}$ and $261 \mathrm{C}>\mathrm{G}$ (circle); $4 \mathrm{C}>\mathrm{T}$ and $77 \mathrm{C}>\mathrm{T}$ (square); $70 \mathrm{~A}>\mathrm{G}$ and 70A>G (triangle); $4 \mathrm{C}>\mathrm{T}$ and -21_-9dup CTCTGTGAAGCTG (diamond)) and three healthy controls (individual gene expression data points for each healthy control and patient are indicated) were trans-differentiated into the chondrogenic lineage by hyperconfluent plating in wells coated with Aggrecan. Total RNA was isolated at days 0 (non-plated fibroblasts), 3, 5 and 7 in trans-differentiation and gene expression of COL2A1 (A), COL1OA1 (B), ALPL (C), RMRP (D), PTHrP (E) and ITS1 (F) was determined by RT-qPCR. Data was normalized to $G A P D H$ and represents the mean plus standard deviation; gene expression data is set relative to day 0 healthy control. For statistical evaluation an independent samples t-test was performed relative to healthy control for each individual time point using GraphPad Prism 5. pvalues are indicated. Graphs are representative examples of three independent experiments. 


\section{Discussion}

The involvement of the RMRP IncRNA in the pathophysiology of $\mathrm{CHH}$ has previously been identified $^{17}$; however it is not clear why mutations in RMRP RNA lead to a severe skeletal dysplasia phenotype. Skeletal development depends on chondrogenic differentiation in the growth plate and therefore we hypothesized that RMRP RNA has a functional role during chondrogenic differentiation, explaining the dwarfism that characterizes $\mathrm{CHH}$ patients. We found that expression levels of RMRP RNA and RNase MRP protein subunits are induced and spatiotemporally regulated during the course of chondrogenic differentiation. RMRP expression levels are especially induced during the hypertrophic phase of chondrogenic differentiation, indicating an increased demand for RMRP RNA levels during this phase of the differentiation process. As long as the substrate(s) of RNase MRP that are involved in the molecular mechanisms of chondrogenic differentiation has/have not been identified, we can only speculate how induction of RMRP RNA expression is associated with chondrocyte hypertrophy. It is remarkable that in $\mathrm{CHH}$ tissues with fast-dividing cell types (growth plate, hair follicles, bone marrow etc.) seem to be the most affected ones ${ }^{16}$, suggesting a role for $R M R P$ RNA in cell cycle regulation. Tight control over cell mitosis and differentiation is paramount to controlling growth plate development ${ }^{47}$. The proliferative zone of the growth plate presents a high mitotic activity, while terminally differentiated chondrocytes in the hypertrophic zone most likely lack a mitotic cycle. One of the identified activities of the RNase MRP complex is endoribonucleolytic cleavage of the mRNA of cyclin b2 (Clb2). This Btype cyclin activates cyclin-dependent kinase-1 (CDK1) during M-phase and anaphasepromoting complex (APC)-dependent proteolysis of $\mathrm{Clb2}$ is essential for mitotic exit ${ }^{48}$. Low Clb2 levels also keep CDK1 activity to a minimum during G0 phase via similar APCdependent proteolysis ${ }^{49}$. It is likely that the strictly regulated terminal differentiation of hypertrophic chondrocytes is orchestrated by mitotic exit or entrance into G0 phase. As RNase MRP activity has been shown to degrade the Clb2 message it is tempting to speculate that the elevation of RNase MRP levels during chondrocyte hypertrophy serves to decrease Clb2 protein levels by increased turnover of Clb2 mRNA to inhibit activation of CDK1. Compelling evidence from recent work indeed shows the crucial involvement of CDK1 in controlling chondrocyte hypertrophic differentiation ${ }^{50}$. CDK1 expression was found to be highly expressed in proliferative chondrocytes and was greatly diminished in hypertrophic 
chondrocytes in differentiating ATDC5 cultures, as well as in mouse growth plates. In addition genetic interference with CDK1 expression caused absence of proliferative chondrocytes in the growth plate and a switch towards hypertrophic differentiation in ATDC5 cultures, strongly suggesting that the reduction of CDK1 levels or its decreased activity is a prerequisite for hypertrophic differentiation. We thus speculate that in a healthy growth plate the increased RMRP RNA levels in hypertrophic chondrocytes may lead to decreased Clb2 levels, thereby hampering CDK1 activity and inducing an overall mitotic arrest. Work from the same group ${ }^{50}$ shows that the proliferation-promoting and hypertrophy-suppressive action of PTHrP is, at least in part, CDK1-dependent. In agreement with this notion we observed that Rmrp RNA levels are down regulated upon PTHrP exposure, presumably via decreased Rmrp promoter transcriptional activity. This may result in elevated Clb2 levels, thereby potentially aiding in PTHrP downstream CDK1 activity. In this respect, it is important to note that knockdown of Rmrp RNA levels during ATDC5 chondrogenic differentiation increases $\mathrm{Clb} 2$ and Pthrp mRNA levels and deregulates chondrogenic differentiation in an overall hypertrophy-suppressing fashion. One of the earliest identified roles of RNase MRP is endoribonucleolytic maturation of 5.8S rRNA by cleaving site $A 3$ in the internal transcribed spacer 1 (ITS1) in yeast ${ }^{3,4}$. Even though there is presently no conclusive evidence showing that specifically hypertrophic chondrocytes in the growth plate display the highest protein synthetic capacity, an RMRP RNA/RNase MRP mediated contribution to the synthesis of the large ribosomal subunit in the growth plate may support the high protein synthetic activity of the growth plate to produce protein-rich cartilaginous extracellular matrix. For long an involvement of RNase MRP in the processing of ITS1 in human cells remained elusive ${ }^{51}$. However, recently it has been elegantly shown that human RMRP RNA in the RNase MRP complex indeed catalyzes the endoribonucleolytic cleavage of ITS1, thereby contributing to pre-rRNA maturation ${ }^{4}$. Indeed our RMRP RNA knockdown data in ATDC5 show accumulation of an ITS1 pre-rRNA processing intermediate as well as reduced levels of mature $5.8 \mathrm{~S}$ and $18 \mathrm{~S}$ rRNA. In concert with these findings we detected similar accumulation of an ITS1 processing intermediate in differentiating $\mathrm{CHH}$ cells. Chondrogenic differentiation of ATDC5 and the developing growth plate is associated with high proliferative capacity ${ }^{31}$ and increased synthesis of protein-rich ECM. It is conceivable that this alters the cellular demand for mature rRNAs for ribosome biogenesis, potentially explaining the changes in Rmrp RNA expression during chondrogenic 
differentiation and the phenotype observed after Rmrp RNA knockdown. Finally, RMRP RNA has been described to associate with $\mathrm{TERT}^{7}$, the reverse transcriptase that is associated with the telomerase holoenzyme. This RMRP RNA-containing macromolecular complex was demonstrated to display RNA-dependent RNA polymerase activity. This activity generates a double-stranded RMRP RNA molecule that is converted by Dicer into an siRNA that targets RMRP RNA ${ }^{7}$, as well as specific mRNAs ${ }^{52}$. Other RMRP RNA-derived siRNAs have been identified as well, but it is currently unknown whether these are also generated via a similar mechanism. The latter siRNAs, termed RMRP-S1 and $R M R P-\mathrm{S}^{8}$, seem to target genes relevant for skeletal development like SOX4, PTCH2 and BMPR2. It remains elusive whether this relates to increased $R M R P$ RNA levels in hypertrophic chondrocytes, but it is conceivable that upregulation of RMRP RNA leads to higher production of RMRP-S1 and $R M R P-S 2$, which in turn may change the magnitude by which RMRP-S1 and RMRP-S2 targets are influenced.

Our observations that the expression of RNase MRP components is modulated in differentiating cells promoted us to investigate whether RMRP RNA expression levels can be controlled at the transcriptional level. RMRP RNA expression is driven by RNA polymerase III (RNAPIII) and the proximal RMRP RNA promoter has been studied in the past to some extent ${ }^{53}$, showing that the $-84 \mathrm{bp}$ promoter region is sufficient to drive RNAPIII-dependent transcription of RMRP RNA. However, to fine-tune promoter activity and to be able to respond to alternating demands of $R M R P$ RNA (e.g. during chondrocyte hypertrophy), it is expected that additional transcription regulatory elements are present. Indeed, WNT-3A was recently found to be able to drive $R M R P$ transcription in cancer via activation of $\beta$ catenin and YAP proteins ${ }^{54}$. Here, we showed that the 1500 base pair sequence upstream of the Rmrp RNA transcription start site is responsive to a series of chondrocyte morphogens or pathways. Several of the growth factors tested increased or decreased the transcriptional activity of the minus 1500 Rmrp promoter, indeed indicating that in chondrocytic cells transcription of Rmrp RNA may be under transcriptional control of chondrogenic cues. In agreement with their known role in chondrocyte hypertrophy ${ }^{45}$, WNT-5A, WNT-3A and BMP-2 induced Rmrp promoter activity, suggesting that increased Rmrp RNA expression in chondrocyte hypertrophy is, at least in part, transcriptionally controlled by one or more of these morphogens and their downstream pathways. Indeed, in silico prediction of putative transcription factor binding sites in the minus 1500 bp Rmrp promoter sequence suggests 
the presence of CREB, MEF2, TCF/LEF and SMAD binding sites (Genomatix; data not shown) which are transcription factors acting downstream of WNT-5A, WNT-3A and BMP-2 signaling. Dorsomorphin (a BMP / SMAD1/5/8 inhibitor) exposure greatly reduced Rmrp promoter activity, further supporting BMP-mediated control of RMRP transcription. In keeping with a potential feedback mechanism it is noteworthy that BMPR2 (the type II BMP receptor) is a potential target of $R M R P-\mathrm{S} 2$, the RMRP RNA-derived siRNA ${ }^{8}$.

Active control over Rmrp transcription in chondrocyte hypertrophy is further substantiated by the observation that PTHrP and bFGF are able to reduce Rmrp promoter activity. PTHrP and bFGF delay chondrocyte hypertrophy/terminal differentiation $23,38,39,55$. At this point we can only speculate whether reduced Rmrp promoter activity and Rmrp expression by PTHrP and bFGF is an indirect result of a morphogen-dependent changing chondrocyte hypertrophic phenotype (e.g. decreased WNT or BMP signaling), or that these morphogens directly control Rmrp abundance by downstream inhibition of Rmrp promoter activity. We conclude that Rmrp transcription in chondrocytes is controlled by well-known chondrogenic signaling pathways that are associated with hypertrophic differentiation. It remains to be determined whether such transcriptional control is also present in other cell types and how this communicates with the RNAPIII transcription machinery ${ }^{56}$.

The clinical presentation of $\mathrm{CHH}$ is dominated by short stature, caused by impaired skeletal development. Our data are consistent with a pivotal role for RMRP RNA during chondrogenic differentiation and is especially linked to chondrocyte hypertrophic differentiation. Genetic interference with Rmrp RNA expression in ATDC5 cells and chondrogenic trans-differentiation of $\mathrm{CHH}$ fibroblasts indicates delayed chondrocyte hypertrophy. Growth plates of $\mathrm{CHH}$ patients are characterized by delayed ossification and the presence of very few hypertrophic chondrocytes ${ }^{25}$. Whether and how delayed chondrocyte hypertrophy may influence the development of the growth plate in $\mathrm{CHH}$ remains to be determined. Finally, considering our observations that the expression of PTHrP is induced during knockdown of Rmrp RNA and in chondrogenic differentiating $\mathrm{CHH}$ fibroblasts and its inhibiting action on RMRP promoter activity and RMRP RNA expression, it is interesting to realize that disrupted PTH/PTHrP signaling is deleterious for skeletal development by endochondral ossification ${ }^{57}$. Considering this it is tempting to speculate whether there are molecular connections between RMRP RNA and PTH/PTHrP in skeletal 
dysplasias like cartilage-hair hypoplasia, metaphyseal chondrodysplasia Jansen type (OMIM \#156400) ${ }^{58}$ and Blomstrand type chondrodysplasia ${ }^{59}$, (OMIM \#215045).

In conclusion, IncRNAs involved in mesenchymal cellular differentiation processes are scarcely known ${ }^{60}$ and we found that the RMRP IncRNA is differentially expressed during different stages of chondrogenic differentiation and displaying a prominent association with chondrocyte hypertrophic development. Our findings shed new light on potential pathobiological mechanisms involved in the skeletal dysplasia phenotype associated with cartilage-hair hypoplasia.

\section{Acknowledgements}

The authors thank Dr. Sidney Altman (Yale University, CT, USA) for generous gifts of antibodies against Rpp25, Rpp30, Rpp38 and Rpp40; Dr. Gregory P. Lunstrum (Shriners Hospital for Children, Portland, OR, USA) for providing the anti-Col10a1 antiserum; Dr. Marianna Tryfonidou (Utrecht University, NL) for technical assistance with IHC; Dr. Brendan Lee (Howard Hughes Medical Institute, Baylor College of Medicine, Houston, TX, USA) for providing the MCT cells; and Dr. Sandy Mattijssen (NICHD, NIH, Bethesda MD, USA) for helpful discussion. This work was financially supported by a bi-lateral cooperation grant (DN 82-304) from the Netherlands Organisation for Scientific Research (NWO) and the Deutsche Forschungsgemeinschaft (DFG) to TW, BZ and EL (SFB1140) and by the Dutch Arthritis Foundation (Reumafonds, grant LLP14 to TW and LVR). BS received funding from NWO (Vidi 91714363) and the Netherlands Heart Foundation (Dekker 2014T105). EL is supported by networks (FACE, MaTrOC) of the German Ministry of Education and Research (BMBF) and the EU Seventh Framework Programme (http://cordis.europa.eu/fp7/home_en.html) through grant 602300 (SYBIL).

\section{Author contributions}

MMFS; conception and design, collection and assembly of data, data analysis and interpretation, manuscript writing, final approval of the manuscript. MMJC, DAMS, WV; collection and assembly of the data, data analysis and interpretation, final approval of the 
manuscript. FF, EL, BZ, BS; provision of study material, manuscript writing, final approval of the manuscript. GJMP, LWvR; conception and design, manuscript writing, final approval of the manuscript. TJMW; conception and design, collection and assembly of data, data interpretation, manuscript writing, final approval of the manuscript.

\section{Competing interests statement}

We have no competing interests to declare. 


\section{References}

1 Welting, T. J., van Venrooij, W. J. \& Pruijn, G. J. Mutual interactions between subunits of the human RNase MRP ribonucleoprotein complex. Nucleic Acids Res 32, 2138-2146, doi:10.1093/nar/gkh539 (2004).

2 Chang, D. D. \& Clayton, D. A. A novel endoribonuclease cleaves at a priming site of mouse mitochondrial DNA replication. EMBO J 6, 409-417 (1987).

3 Lygerou, Z., Allmang, C., Tollervey, D. \& Seraphin, B. Accurate processing of a eukaryotic precursor ribosomal RNA by ribonuclease MRP in vitro. Science 272, 268270 (1996).

4 Goldfarb, K. C. \& Cech, T. R. Targeted CRISPR disruption reveals a role for RNase MRP RNA in human preribosomal RNA processing. Genes Dev 31, 59-71, doi:10.1101/gad.286963.116 (2017).

5 Gill, T., Cai, T., Aulds, J., Wierzbicki, S. \& Schmitt, M. E. RNase MRP cleaves the CLB2 mRNA to promote cell cycle progression: novel method of mRNA degradation. $\mathrm{Mol}$ Cell Biol 24, 945-953 (2004).

6 Mattijssen, S. et al. Viperin mRNA is a novel target for the human RNase MRP/RNase P endoribonuclease. Cell Mol Life Sci 68, 2469-2480, doi:10.1007/s00018-010-0568-3 (2011).

7 Maida, Y. et al. An RNA-dependent RNA polymerase formed by TERT and the RMRP RNA. Nature 461, 230-235, doi:10.1038/nature08283 (2009).

8 Rogler, L. E. et al. Small RNAs derived from IncRNA RNase MRP have gene-silencing activity relevant to human cartilage-hair hypoplasia. Hum Mol Genet 23, 368-382, doi:10.1093/hmg/ddt427 (2014).

9 Huang, W. et al. DDX5 and its associated IncRNA Rmrp modulate TH17 cell effector functions. Nature 528, 517-522, doi:10.1038/nature16193 (2015).

10 Welting, T. J., Kikkert, B. J., van Venrooij, W. J. \& Pruijn, G. J. Differential association of protein subunits with the human RNase MRP and RNase P complexes. RNA 12, 1373-1382, doi:10.1261/rna.2293906 (2006).

11 Robertson, H. D., Altman, S. \& Smith, J. D. Purification and properties of a specific Escherichia coli ribonuclease which cleaves a tyrosine transfer ribonucleic acid presursor. J Biol Chem 247, 5243-5251 (1972).

12 Reiner, R., Krasnov-Yoeli, N., Dehtiar, Y. \& Jarrous, N. Function and assembly of a chromatin-associated RNase $P$ that is required for efficient transcription by RNA polymerase I. PLoS One 3, e4072, doi:10.1371/journal.pone.0004072 (2008).

Reiner, R., Ben-Asouli, Y., Krilovetzky, I. \& Jarrous, N. A role for the catalytic ribonucleoprotein RNase P in RNA polymerase III transcription. Genes Dev 20, 16211635, doi:10.1101/gad.386706 (2006).

14 Mattijssen, S., Welting, T. J. \& Pruijn, G. J. RNase MRP and disease. Wiley Interdiscip Rev RNA 1, 102-116, doi:10.1002/wrna.9 (2010). 
Thiel, C. T. et al. Severely incapacitating mutations in patients with extreme short stature identify RNA-processing endoribonuclease RMRP as an essential cell growth regulator. Am J Hum Genet 77, 795-806, doi:10.1086/497708 (2005).

Makitie, O. \& Kostjukovits, S. in GeneReviews(R) (eds R. A. Pagon et al.) (1993).

Ridanpaa, M. et al. Mutations in the RNA component of RNase MRP cause a pleiotropic human disease, cartilage-hair hypoplasia. Cell 104, 195-203 (2001).

Shiasi Arani, K. Clinical features and management of Cartilage-Hair Hypoplasia: a narrative review. J Pediatr Rev 3(1):e194, 8, doi:10.5812/jpr.194 (2015).

Park, J. et al. Dual pathways to endochondral osteoblasts: a novel chondrocytederived osteoprogenitor cell identified in hypertrophic cartilage. Biol Open 4, 608621, doi:10.1242/bio.201411031 (2015).

0 Yang, G. et al. Osteogenic fate of hypertrophic chondrocytes. Cell Res 24, 1266-1269, doi:10.1038/cr.2014.111 (2014).

1 Zhou, X. et al. Chondrocytes transdifferentiate into osteoblasts in endochondral bone during development, postnatal growth and fracture healing in mice. PLOS Genet 10, e1004820, doi:10.1371/journal.pgen.1004820 (2014).

2 Mackie, E. J., Ahmed, Y. A., Tatarczuch, L., Chen, K. S. \& Mirams, M. Endochondral ossification: how cartilage is converted into bone in the developing skeleton. Int J Biochem Cell Biol 40, 46-62, doi:10.1016/j.biocel.2007.06.009 (2008).

Kronenberg, H. M. Developmental regulation of the growth plate. Nature 423, 332336, doi:10.1038/nature01657 (2003).

4 Bonafe, L. et al. Nosology and classification of genetic skeletal disorders: 2015 revision. Am J Med Genet A 167A, 2869-2892, doi:10.1002/ajmg.a.37365 (2015).

25 Hermanns, P., Zabel, B. \& Lee, B. RMRP and Cartilage-Hair Hypoplasia. Epstein's Inborn Errors of Development: The Molecular Basis of Clinical Disorders of Morphogenesis (Oxford Monographs on Medical Genetics), 1142-1148 (2007).

6 Lunstrum, G. P. et al. Chondrocyte differentiation in a rat mesenchymal cell line. J Histochem Cytochem 47, 1-6 (1999).

27 Guerrier-Takada, C., Eder, P. S., Gopalan, V. \& Altman, S. Purification and characterization of Rpp25, an RNA-binding protein subunit of human ribonuclease $P$. RNA 8, 290-295 (2002).

28 Eder, P. S., Kekuda, R., Stolc, V. \& Altman, S. Characterization of two scleroderma autoimmune antigens that copurify with human ribonuclease P. Proc Natl Acad Sci U S A 94, 1101-1106 (1997).

Jarrous, N., Eder, P. S., Guerrier-Takada, C., Hoog, C. \& Altman, S. Autoantigenic properties of some protein subunits of catalytically active complexes of human ribonuclease P. RNA 4, 407-417 (1998).

Lygerou, Z., Pluk, H., van Venrooij, W. J. \& Seraphin, B. hPop1: an autoantigenic protein subunit shared by the human RNase P and RNase MRP ribonucleoproteins. EMBO J 15, 5936-5948 (1996). 
31 Atsumi, T., Miwa, Y., Kimata, K. \& Ikawa, Y. A chondrogenic cell line derived from a differentiating culture of AT805 teratocarcinoma cells. Cell Differ Dev 30, 109-116 (1990).

32 Lefebvre, V., Garofalo, S. \& de Crombrugghe, B. Type X collagen gene expression in mouse chondrocytes immortalized by a temperature-sensitive simian virus 40 large tumor antigen. J Cell Biol 128, 239-245 (1995).

Caron, M. M. et al. Activation of NF-kappaB/p65 facilitates early chondrogenic differentiation during endochondral ossification. PLoS One 7, e33467, doi:10.1371/journal.pone.0033467 (2012).

34 French, M. M., Rose, S., Canseco, J. \& Athanasiou, K. A. Chondrogenic differentiation of adult dermal fibroblasts. Ann Biomed Eng 32, 50-56 (2004).

Caron, M. M. et al. BAPX-1/NKX-3.2 acts as a chondrocyte hypertrophy molecular switch in osteoarthritis. Arthritis Rheumatol 67, 2944-2956, doi:10.1002/art.39293 (2015).

36 Caron, M. M. et al. Hypertrophic differentiation during chondrogenic differentiation of progenitor cells is stimulated by BMP-2 but suppressed by BMP-7. Osteoarthritis Cartilage 21, 604-613, doi:10.1016/j.joca.2013.01.009 (2013).

37 Yamashita, S. et al. Sox9 directly promotes Bapx1 gene expression to repress Runx2 in chondrocytes. Exp Cell Res 315, 2231-2240, doi:10.1016/j.yexcr.2009.03.008 (2009).

Guo, J. et al. PTH/PTHrP receptor delays chondrocyte hypertrophy via both Runx2dependent and -independent pathways. Dev Biol 292, 116-128, doi:10.1016/j.ydbio.2005.12.044 (2006).

39 Terkeltaub, R. A. et al. Bone morphogenetic proteins and bFGF exert opposing regulatory effects on PTHrP expression and inorganic pyrophosphate elaboration in immortalized murine endochondral hypertrophic chondrocytes (MCT cells). J Bone Miner Res 13, 931-941, doi:10.1359/jbmr.1998.13.6.931 (1998).

40 Huang, F. \& Chen, Y. G. Regulation of TGF-beta receptor activity. Cell Biosci 2, 9, doi:10.1186/2045-3701-2-9 (2012).

41 Cals, F. L., Hellingman, C. A., Koevoet, W., Baatenburg de Jong, R. J. \& van Osch, G. J. Effects of transforming growth factor-beta subtypes on in vitro cartilage production and mineralization of human bone marrow stromal-derived mesenchymal stem cells. J Tissue Eng Regen Med 6, 68-76, doi:10.1002/term.399 (2012).

$42 \mathrm{Wu}, \mathrm{J}$. et al. Mechanism and in vitro pharmacology of TAK1 inhibition by (5Z)-7Oxozeaenol. ACS Chem Biol 8, 643-650, doi:10.1021/cb3005897 (2013).

Sanvitale, C. E. et al. A new class of small molecule inhibitor of BMP signaling. PLoS One 8, e62721, doi:10.1371/journal.pone.0062721 (2013).

Coleman, C. M. et al. Growth differentiation factor-5 enhances in vitro mesenchymal stromal cell chondrogenesis and hypertrophy. Stem Cells Dev 22, 1968-1976, doi:10.1089/scd.2012.0282 (2013). 
Studer, D., Millan, C., Ozturk, E., Maniura-Weber, K. \& Zenobi-Wong, M. Molecular and biophysical mechanisms regulating hypertrophic differentiation in chondrocytes and mesenchymal stem cells. Eur Cell Mater 24, 118-135; discussion 135 (2012).

6 Yang, J., Andre, P., Ye, L. \& Yang, Y. Z. The Hedgehog signalling pathway in bone formation. Int J Oral Sci 7, 73-79, doi:10.1038/ijos.2015.14 (2015).

7 Beier, F. Cell-cycle control and the cartilage growth plate. J Cell Physiol 202, 1-8, doi:10.1002/jcp.20111 (2005).

8 Wasch, R. \& Cross, F. R. APC-dependent proteolysis of the mitotic cyclin Clb2 is essential for mitotic exit. Nature 418, 556-562, doi:10.1038/nature00856 (2002).

Guardavaccaro, D. \& Pagano, M. Stabilizers and destabilizers controlling cell cycle oscillators. Mol Cell 22, 1-4, doi:10.1016/j.molcel.2006.03.017 (2006).

0 Saito, M. et al. The Indispensable Role of Cyclin-Dependent Kinase 1 in Skeletal Development. Sci Rep 6, 20622, doi:10.1038/srep20622 (2016).

5 Sloan, K. E. et al. Both endonucleolytic and exonucleolytic cleavage mediate ITS1 removal during human ribosomal RNA processing. J Cell Biol 200, 577-588, doi:10.1083/jcb.201207131 (2013).

52 Maida, Y., Kyo, S., Lassmann, T., Hayashizaki, Y. \& Masutomi, K. Off-target effect of endogenous siRNA derived from RMRP in human cells. Int J Mol Sci 14, 9305-9318, doi:10.3390/ijms14059305 (2013).

53 Yuan, Y. \& Reddy, R. 5 ' flanking sequences of human MRP/7-2 RNA gene are required and sufficient for the transcription by RNA polymerase III. Biochim Biophys Acta 1089, 33-39 (1991).

4 Park, J. \& Jeong, S. Wht activated beta-catenin and YAP proteins enhance the expression of non-coding RNA component of RNase MRP in colon cancer cells. Oncotarget 6, 34658-34668, doi:10.18632/oncotarget.5778 (2015).

$5 \mathrm{Su}$, N., Jin, M. \& Chen, L. Role of FGF/FGFR signaling in skeletal development and homeostasis: learning from mouse models. Bone Res 2, 14003, doi:10.1038/boneres.2014.3 (2014).

6 Orioli, A., Pascali, C., Pagano, A., Teichmann, M. \& Dieci, G. RNA polymerase III transcription control elements: themes and variations. Gene 493, 185-194, doi:10.1016/j.gene.2011.06.015 (2012).

57 Schipani, E. \& Provot, S. PTHrP, PTH, and the PTH/PTHrP receptor in endochondral bone development. Birth Defects Res C Embryo Today 69, 352-362, doi:10.1002/bdrc.10028 (2003).

58 Savoldi, G. et al. Prenatal presentation and postnatal evolution of a patient with Jansen metaphyseal dysplasia with a novel missense mutation in PTH1R. Am J Med Genet A 161A, 2614-2619, doi:10.1002/ajmg.a.36115 (2013).

Jobert, A. S. et al. Absence of functional receptors for parathyroid hormone and parathyroid hormone-related peptide in Blomstrand chondrodysplasia. J Clin Invest 102, 34-40, doi:10.1172/JCI2918 (1998). 
Chapter 2

60 Hassan, M. Q., Tye, C. E., Stein, G. S. \& Lian, J. B. Non-coding RNAs: Epigenetic regulators of bone development and homeostasis. Bone 81, 746-756, doi:10.1016/j.bone.2015.05.026 (2015). 
Expression of RMRP RNA is regulated in chondrocyte hypertrophy and determines chondrogenic differentiation 
1 Laboratory for Experimental Orthopedics, Department of Orthopedic Surgery, Maastricht University Medical Center, Maastricht, the Netherlands

2 Department of Anatomy and Embryology, Maastricht University, Maastricht, the Netherlands

${ }^{3}$ University Heart Centre Freiburg, Faculty of Medicine, University of Freiburg, Institute for Experimental Cardiovascular Medicine, Freiburg, Germany

4 Medical Faculty, Otto van Guericke University of Magdeburg, Magdeburg, Germany

${ }^{5}$ Department of Musculoskeletal Biology, Institute of Ageing and Chronic Disease, University of Liverpool, Liverpool, United Kingdom

* Authors contributed equally 


\section{Chapter 3}

\section{The anti-viral protein viperin regulates chondrogenic differentiation via CXCL10 protein secretion}

Mandy M.F. Steinbusch', Marjolein M.J. Caron', Don A.M. Surtel', Guus G.H. van den Akker' , Paul J. van Dijk ${ }^{2}$, Franziska Friedrich ${ }^{3}$, Bernhard Zabel ${ }^{4}$, Lodewijk W. van Rhijn', Mandy J. Peffers ${ }^{5 *}$, Tim J.M. Welting ${ }^{1 *}$

Published in Journal of Biological Chemistry: J Biol Chem (2019) 294 (13) 5121-5136, doi: 10.1074/jbc.RA1 19.007356 


\section{Abstract}

Viperin (also known as radical SAM domain-containing 2, RSAD2) is an interferon-inducible and evolutionary conserved protein that participates in the cell's innate immune response against a number of viruses. Viperin mRNA is a substrate for endoribonucleolytic cleavage by RNase mitochondrial RNA processing (MRP) and mutations in the RMRP small nucleolar RNA (snoRNA) subunit of the RNase MRP complex cause cartilage-hair hypoplasia $(\mathrm{CHH})$, a human developmental condition characterized by metaphyseal chondrodysplasia and severe dwarfism. It is unknown how CHH-pathogenic mutations in RMRP snoRNA interfere with skeletal development and aberrant processing of RNase MRP substrate RNAs is thought to be involved. We hypothesized that viperin plays a role in chondrogenic differentiation. Using immunohistochemistry, RT-qPCR, immunoblotting, ELISA, siRNA-mediated gene silencing, plasmid-mediated gene overexpression, label-free mass-spectrometry proteomics and promoter reporter bioluminescence assays, we discovered here that viperin is expressed in differentiating chondrocytic cells and regulates their protein secretion and the outcome of chondrogenic differentiation by influencing transforming growth factor $\beta$ (TGFB)/SMAD family $2 / 3$ (SMAD2/3) activity via C-X-C motif chemokine ligand 10 (CXCL10). Of note, we observed disturbances in this viperin-CXCL10-TGF- $\beta /$ SMAD2/3 axis in $\mathrm{CHH}$ chondrocytic cells. Our results indicate that the anti-viral protein viperin controls chondrogenic differentiation by influencing secretion of soluble proteins and identify a molecular route that may explain impaired chondrogenic differentiation of cells from individuals with $\mathrm{CHH}$. 


\section{Introduction}

Viperin, also known as RSAD2, is an interferon-inducible and evolutionary conserved protein that participates in the cell's innate immune response against a number of viruses. Viperin localizes to the cytosolic face of the endoplasmic reticulum (ER), mitochondria and lipid droplets. It is described to exert its antiviral properties via several pathways, including inhibition of soluble protein secretion, alterations of mitochondrial energy metabolism, inhibition of virus replication in lipid droplets and modulation of cellular signaling events ${ }^{1}$. The mRNA of viperin was identified as a substrate for endoribonucleolytic cleavage by the RNase MRP small nucleolar ribonucleoprotein complex ${ }^{2}$. Mutations in the RMRP gene, which encodes the essential small nucleolar (snoRNA) subunit of the RNase MRP complex, are known to be the cause of the human genetic disease cartilage-hair hypoplasia ${ }^{3,4}(\mathrm{CHH}$ : OMIM \#250250). A major phenotypic hallmark of $\mathrm{CHH}$ is impaired skeletal development characterized by metaphyseal chondrodysplasia, leading to a severe form of dwarfism ${ }^{5}$. Growth plates are central drivers in the formation of the skeleton and their endochondral ossification depends on a tightly orchestrated continuing process of chondrogenic differentiation, ultimately resulting in longitudinal growth of the long bones ${ }^{6}$. Although a number of RNase MRP substrates have been identified in man $^{2,4,7-9}$, including viperin, there is a major lack of studies investigating the role of RNase MRP substrate RNAs in development, and in chondrogenic differentiation in particular. It is expected that insight into the involvement of RNase MRP substrates in chondrogenic differentiation will provide insight into the molecular mechanism leading to impaired skeletal development in $\mathrm{CHH}$. In concert with viperin mRNA as a substrate for RNase MRP cleavage, viperin expression levels have been described to be increased after knockdown of protein components of the RNase MRP complex in HEp-2 cells ${ }^{2}$, in leukocytes of $\mathrm{CHH}$ patients ${ }^{10}$ and recently we demonstrated that viperin expression is also upregulated after knockdown of RMRP RNA in chondrogenic differentiating ATDC5 cells ${ }^{11}$. Since increased viperin expression as a result of interference with RNase MRP function appears to be conserved amongst several cell types, we hypothesized that viperin regulates the course of chondrogenic differentiation. In this study we examined the expression of viperin, determined its role in chondrocyte protein secretion, and investigated its crosstalk with the TGF- $\beta / S M A D 2 / 3$ signaling pathway in chondrogenic differentiation. We discovered that viperin is expressed in differentiating 
chondrocytic cells, regulates their protein secretion and impacts the outcome of the chondrogenic differentiation program through influencing TGF- $\beta / S M A D 2 / 3$ activity via CXCL10. Our data for the first time shows that the anti-viral protein viperin regulates chondrogenic differentiation by influencing the secretion of soluble proteins and highlights its involvement in impaired chondrogenic differentiation in $\mathrm{CHH}$ patient cells.

\section{Experimental procedures}

\section{Immunohistochemistry (IHC)}

Five micrometer-thick formalin-fixed paraffin-embedded tissue sections were prepared from E15.5 NMRI mouse embryos. Use of the embryos was approved by the University of Freiburg (number X-14/10H), according to German law; and methods utilized to obtain the embryos were carried out in accordance with German law. Sections were deparaffinized in a xylene / ethanol series ending in PBS (136 mM NaCl (Merck Millipore, Darmstadt, Germany),

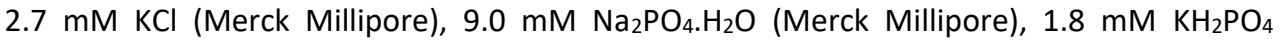
(Merck Millipore)). For antigen retrieval, sections were incubated in hot citrate buffer (1.8 $\mathrm{mM}$ citric acid (Sigma-Aldrich, St. Louis, MO, USA) and $8.2 \mathrm{mM}$ tri-sodium citrate (VWR Prolabo, Amsterdam, the Netherlands)) for 30 minutes. Endogenous peroxidase activity was inactivated using peroxidase-blocking solution (Dako, Troy, MI, USA). Slides were blocked with $10 \%$ normal sheep serum in PBS-T (0.1\% Tween 20; Sigma-Aldrich). Primary antibody was incubated for 2 hours at room temperature. Mouse monoclonal anti-Viperin (Merck Millipore \#MABF106) was used at a 1:200 dilution. IgG2a (Dako) was used as an isotype control. Bound antibodies were detected with horseradish peroxidase (HRP)-conjugated rabbit anti-mouse secondary antibodies (Dako EnVision) for 30 minutes at room temperature. DAB chromogen substrate (Dako) was used for detection. Sections were counterstained with haematoxylin (Dako), dehydrated and mounted with histomount (Thermo Shandon, Waltham, MA, USA). Microphotographs were acquired using a Zeiss Axioscope A1. 


\section{Cell culture and differentiation of ATDC5}

ATDC5 cells ${ }^{12}$ (RIKEN BRC, Japan) were cultured in a humidified atmosphere at $37^{\circ} \mathrm{C}, 5 \% \mathrm{CO}_{2}$ in proliferation medium (DMEM/F12 (Invitrogen, Carlsbad, CA, USA), 5\% fetal calf serum (FCS) (Sigma-Aldrich), 1\% antibiotic/antimycotic (Invitrogen), 1\% non-essential amino acids (NEAA) (Invitrogen)). Cells were tested negative for potential mycoplasma infection. To induce chondrogenic differentiation, cells were plated at 6,400 cells $/ \mathrm{cm}^{2}$. After 24 hours, chondrogenic differentiation was initiated by changing the medium to differentiation medium (proliferation medium supplemented with $10 \mu \mathrm{g} / \mathrm{ml}$ insulin (Sigma-Aldrich), 10 $\mu \mathrm{g} / \mathrm{ml}$ transferrin (Roche, Basel, Switzerland), $30 \mathrm{nM}$ sodium selenite (Sigma-Aldrich)). Differentiation medium was refreshed every two days for the first 10 days, and each day after day 10. In addition, ATDC5 cells were differentiated for 5 days and then exposed to 0.5 or $5 \mathrm{ng} / \mathrm{ml}$ recombinant murine CXCL10 (Peprotech, Rocky Hill, NJ, USA, \#250-16) until day 7 in differentiation. When ATDC5 cells were differentiated using conditioned media (CM), the $\mathrm{CM}$ was refreshed every other day.

\section{Cell culture and differentiation of hBMSCs}

Human bone marrow stem cells (hBMSCs) were obtained from residual iliac crest bone marrow aspirate from young, genetically healthy, individuals undergoing spinal surgery. Approval from the Maastricht University Medical Center Medical Ethical Committee (MEC) for the use of this material was obtained and assigned approval ID: MEC 08-4-056. Human BMSCs from four individual donors were isolated from the aspirate using Ficoll Paque (Amersham). Proliferation medium consisted of DMEM high-glucose (Invitrogen), 10\% FCS (ES-grade), 1\% antibiotic/antimycotic and 1\% NEAA. Passage 3 cells were plated at 30,000 cells $/ \mathrm{cm}^{2}$ in quadruplicates and chondrogenic differentiation was initiated the next day by changing to differentiation medium (proliferation medium supplemented with $1 \%$ ITS (Invitrogen), $50 \mu \mathrm{g} / \mathrm{ml}$ L-ascorbic acid-2-phosphate (Sigma) and $1 \mathrm{ng} / \mathrm{ml}$ TGFß3 (R\&D)) ${ }^{13}$. Medium was changed every 2 days. Human CXCL10 (Peprotech, Rocky Hill, NJ, USA) was used at indicated concentrations $(0.5,5,50,200 \mathrm{ng} / \mathrm{ml})$. Cells were harvested in TRIzol at day $0,2,7,14$ and 21 in differentiation for RNA isolation. 


\section{Real time quantitative PCR (RT-qPCR)}

RNA was extracted from cells with TRIzol reagent (Invitrogen) and isolated by collecting the aqueous phase after phase separation. RNA was precipitated with isopropanol (30 minutes, $-80^{\circ} \mathrm{C}$ ) and centrifuged for 30 minutes at 15,000 RPM, $4^{\circ} \mathrm{C}$. RNA pellets were washed with $80 \%$ ethanol and potential DNA contamination was removed by DNase I (Roche) treatment $\left(1\right.$ hour, $\left.37^{\circ} \mathrm{C}\right)$. After subsequent ethanol precipitation, RNA was dissolved in $15 \mu \mathrm{L}$ DNase/RNase free water (Eurogentec, Seraing, Belgium). RNA quantity and purity were determined spectrophotometrically (Biodrop, Isogen Life Sciences, Utrecht, the Netherlands). Reverse transcription of RNA and RT-qPCR were performed as described previously ${ }^{11}$. Housekeeping genes were $\beta$-Actin for ATDC5 and CYCLOPHILIN for hBMSCs and human fibroblasts. Primer sequences are provided in Table 1.

Table 1. RT-qPCR primer sequences

\begin{tabular}{|l|l|l|}
\hline Gene & Forward & Reverse \\
\hline ALPL $(\mathrm{Hs})$ & CCGTGGCAACTCTATCTTTGG & CAGGCCCATTGCCATACAG \\
\hline Alpl $(\mathrm{Mm})$ & CCGATGGCACACCTGCTT & GGAGGCATACGCCATCACAT \\
\hline B-Actin $(\mathrm{Mm})$ & CCGAGCGCGAGATCGT & TGGCCATCTCGTTCTCGAA \\
\hline COL2A1 $(\mathrm{Hs})$ & TGGGTGTTCTATTTATTTATTGTCTTCCT & GCGTTGGACTCACACCAGTTAGT \\
\hline Col2a1 $(\mathrm{Mm})$ & TGGGTGTTCTATTTATTTATTGTCTTCCT & GCGTTGGACTCACACCAGTTAGT \\
\hline COL10A1 $(\mathrm{Hs})$ & ATGATGAATACACCAAAGGCTACCT & ACGCACACCTGGTCATTTTCTG \\
\hline Col10a1 $(\mathrm{Mm})$ & CATGCCTGATGGCTTCATAAA & AAGCAGACACGGGCATACCT \\
\hline CYCLOPHILIN $(\mathrm{Hs})$ & TTCCTGCTTTCACAGAATTATTCC & GCCACCAGTGCCATTATGG \\
\hline PAl1 $(\mathrm{Hs})$ & GTCTGCTGTGCACCATCCCCCATC & TTGTCATCAATCTTGAATCCCATA \\
\hline Pai1 $(\mathrm{Mm})$ & ACGTCGTGGAACTGCCCTAC & CAGCGATGAACATGCTGAGG \\
\hline RMRP $(\mathrm{Hs})$ & GAGAGTGCCACGTGCATACG & ACGCTTCTTGGCGGACTTT \\
\hline Rmrp $(\mathrm{Mm})$ & ATACGAGGGACATGTTCCTTATCC & TTGGCGGGCTAACAGTGACT \\
\hline RUNX2 $(\mathrm{Hs})$ & TGATGACACTGCCACCTCTG & GCACCTGCCTGGCTCTTCT \\
\hline Runx2 $(\mathrm{Mm})$ & GACGAGGCAAGAGTTTCACC & GGACCGTCCACTGTCACTTT \\
\hline SMAD7 $(\mathrm{Hs})$ & CCTTAGCCGACTCTGCGAACTA & CCAGATAATTCGTTCCCCCTGT \\
\hline Smad7 $(\mathrm{Mm})$ & GCAACCCCCATCACCTTAGTC & GTTTGAGAAAATCCATTGGGTATCTG \\
\hline SOX9 $(\mathrm{Hs})$ & AGTACCCGCACCTGCACAAC & CGCTTCTCGCTCTCGTTCAG \\
\hline Sox9 $(\mathrm{Mm})$ & AGTACCCGCACCTGCACAAC & TACTTGTAGTCCGGGTGGTCTTTC \\
\hline
\end{tabular}




\begin{tabular}{|l|l|l|}
\hline Gene & Forward & Reverse \\
\hline VIPERIN $(\mathrm{Hs})$ & CCAACCAGCGTCAACTATCACTT & GGAAACAGAAGCCGCATTTG \\
\hline Viperin $(\mathrm{Mm})$ & TGCTATCTCCTGCGACAGCTT & CCTTGACCACGGCCAATC \\
\hline
\end{tabular}

Primer sequences were designed for Mus musculus ( $\mathrm{Mm}$ ) or Homo sapiens (Hs).

\section{Immunoblotting}

Cells were washed three times with $0.9 \% \mathrm{NaCl}$. The cells were lysed in RIPA buffer $(150 \mathrm{mM}$ $\mathrm{NaCl}, 1 \% \mathrm{NP}-40,0.5 \%$ sodium deoxycholate, $0.1 \% \mathrm{SDS}, 50 \mathrm{mM}$ Tris $\mathrm{pH}$ 8.0, $5.0 \mathrm{mM}$ EDTA pH 8.0, $0.5 \mathrm{mM}$ dithiothreitol (DTT), $1 \mathrm{mM}$ phenylmethylsulfonylfluoride (PMSF) and phosphatase inhibitor (PhosSTOP; Roche)). Samples were homogenized by sonication (Soniprep $150 \mathrm{MSE}$ ) using the following protocol: 14 cycles of 1 second sonication followed by a 1 second interval, amplitude 10. Cell debris was removed by centrifugation. Total protein concentration was determined with a BCA assay (Sigma-Aldrich). Samples were separated by gel electrophoresis and transferred to nitrocellulose membranes by electroblotting. The following primary antibodies were used for immuno-detection: Mouse monoclonal anti-Viperin (Merck Millipore \#MABF106; 1:500 dilution) and rabbit monoclonal anti-pSMAD2C (Cell Signaling Technologies \#3108; 1:1000 dilution). As controls mouse monoclonal anti-Histone H3 (Abcam \#24834; 1:1000 dilution), mouse monoclonal antialpha-Tubulin (Sigma-Aldrich \#T6074; 1:10,000 dilution) and mouse monoclonal anti-GAPDH (Fitzgerald \#10R-G109b; 1:5000 dilution) were used. HRP-conjugated polyclonal rabbit antimouse (Dako) or HRP-conjugated swine anti-rabbit (Dako) was applied as a secondary antibody and the bound antibodies were detected by enhanced chemiluminescence (ECL).

\section{Transfection of siRNAs}

Chondrogenic differentiating ATDC5 cells were transfected (Pepmute; SignaGen Laboratories, Rockville, MD, USA; according to the manufacturer's protocol) with $100 \mathrm{nM}$ siRNA duplexes (custom synthesized by Eurogentec, see Table 2) targeting Viperin (on day 3 or day 5 of chondrogenic differentiation) or Rmrp RNA (on day $-1,2$, and 5 of chondrogenic 
differentiation). Scrambled siRNA duplex was purchased from Eurogentec; REF: SR-CLO00005.

Table 2. siRNA sequences

\begin{tabular}{|l|l|l|}
\hline siRNA & Sense & Anti-sense \\
\hline Viperin siRNA & GGGAAGCAGAAAGAUUUCUdTdT & AGAAAUCUUUCUGCUUCCCdTdT \\
\hline Rmrp siRNA & CAUGUUCCUUAUCCUUUCGdTdT & CGAAAGGAUAAGGAACAUGdTdT \\
\hline
\end{tabular}

\section{Plasmids and transfection}

The viperin coding sequence, flanked by Notl - Xbal restriction sites, was custom-made synthesized by GeneCust (Ellange, Luxembourg) and originally cloned in pUC57 vector and sequence verified. The insert was then sub-cloned into the p3xFLAG plasmid (Promega, Southampton, UK) using the NotI - Xbal restriction sites, yielding the viperin overexpression plasmid (FLAG-viperin). On day 4, 5 or 6 of ATDC5 chondrogenic differentiation, viperin expression was further induced in 6-well plates by transfection of $625 \mathrm{ng}$ FLAG-viperin plasmid or an empty plasmid (FLAG-empty) as a control, using polyethylenimine (PEI; Polysciences, Warrington, PA, USA) transfection reagent. DNA and PEI were complexed for 10 minutes at room temperature in DMEM/F12 $(1.88 \mu \mathrm{l} \mathrm{PEI}(1 \mu \mathrm{g} / \mu \mathrm{l})$ per $625 \mathrm{ng}$ construct per well) and added to the ATDC5 cells. To determine protein secretion $250 \mathrm{ng}$ CMV promoter-driven secreted Gaussia luciferase plasmid (pGluc-CMV ${ }^{14}$ ) was co-transfected with 200 ng CMV promoter-driven firefly luciferase pGL4.20-CMV (control; Promega) on day 3 or day 5 of ATDC5 chondrogenic differentiation (6-well format), using PEI transfection reagent. Culture supernatant and cells were collected for bioluminescence analyses and Gaussia bioluminescence was normalized to the firefly signal. Viperin levels were either reduced or overexpressed in differentiating ATDC5 cultures from day 5 to day 7 and conditioned culture supernatants were collected. Subsequently, proliferating ATDC5 cells were used as a bioassay for SMAD3-dependent TGF $\beta$ activity by transfection with 500 ng CAGA12-luciferase reporter plasmid ${ }^{15}$ (kind gift of Dr. Dalmay ${ }^{16}$ ) and $125 \mathrm{ng}$ pGluc-CMV ${ }^{14}$ plasmid as a transfection control, using PEI transfection reagent. The proliferating ATDC5 cells were then exposed to the above mentioned conditioned culture supernatants for 24 hours to determine the TGFB/SMAD3-activity, where firefly bioluminescence was normalized to the 
Gaussia signal. The action of exogenously added CXCL10 on SMAD3-dependent TGF- $\beta$ activity during chondrogenic differentiation was determined by transfecting day 5differentiating ATDC5 cells in 6-well format with 500 ng CAGA12-luciferase reporter and 125 ng pGluc-CMV plasmid as a transfection control, using PEI transfection reagent. Twenty-four hours later cells were exposed to $0.5,5,20$ or $200 \mathrm{ng} / \mathrm{ml}$ recombinant murine CXCL10 (Peprotech \#250-16). At day 7 in differentiation, culture supernatant and cells were then collected for bioluminescence analyses and firefly bioluminescence was normalized to the Gaussia signal. pGluc-CMV (Gaussia luciferase), pGL4.20-CMV (firefly luciferase) and CAGA12 (firefly luciferase) samples were all harvested for bioluminescence detection using the Dual Luciferase reporter assay system (Promega) as described by the manufacturer and measured on a Fluostar Omega plate reader (BMG Labtech, Ortenberg, Germany).

\section{Alcian Blue and Crystal Violet staining and quantification}

Cells were washed twice with $0.9 \% \mathrm{NaCl}$ and fixated with $4 \%$ paraformaldehyde in PBS for 10 minutes at room temperature. Fixated cells were washed 6 times with distilled water and air-dried. Dried fixated cells were incubated for 30 minutes at room temperature with respectively $1 \%(\mathrm{~m} / \mathrm{v}$ ) Alcian Blue (Acros Organics, Geel, Belgium) in $0.1 \mathrm{M} \mathrm{HCl}$ or $0.1 \%(\mathrm{~m} / \mathrm{v}$ ) Crystal Violet (Sigma-Aldrich). Cells were washed six times with distilled water and allowed to air dry. Alcian Blue was extracted by incubation with $6 \mathrm{M}$ Guanidine- $\mathrm{HCl}$ (Sigma-Aldrich) for 2 hours on a plate shaker (IKA HS 260 Basic, IKA, Staufen, Germany). Crystal Violet was extracted by incubation with $10 \%$ acetic acid (VWR) for 15 minutes on a plate shaker (IKA). Extracted Alcian Blue and Crystal Violet were quantified spectrophotometrically at $645 \mathrm{~nm}$ or $590 \mathrm{~nm}$ respectively using a plate reader (ThermoScientific Multiskan FC, Waltham, MA, USA). Crystal Violet (DNA content) was used as normalization for Alcian Blue glycosaminoglycan (GAG) content. 


\section{In-solution tryptic digestion and mass spectrometry proteomics of media following viperin knockdown or overexpression}

At day 5 in ATDC5 chondrogenic differentiation, viperin expression was either reduced by transfection of a viperin-specific siRNA duplex or a scrambled control siRNA duplex; or viperin expression was further induced by transfection of the FLAG-viperin plasmid or the empty FLAG plasmid. Cells were then further differentiated until day 7 and conditioned medium (CM) was collected. During differentiation phenol-red free DMEM-F12 (Invitrogen) was used in order to accommodate mass spectrometry analysis. To obtain sufficient amounts of $\mathrm{CM}$, the experiment was simultaneously performed in three triplicates for each condition. Triplicates were then pooled to obtain three times scrambled siRNA duplex, three times viperin siRNA duplex, three times empty FLAG and three times FLAG-viperin overexpression $\mathrm{CM}$ samples. Two representative triplicates from each condition were utilized to confirm viperin knockdown and overexpression by means of immunoblotting. The CM was centrifuged for 5 minutes at $1200 \mathrm{rpm}$, to remove dead cells, and subsequently for 5 minutes at $13,000 \mathrm{rpm}$ to remove remaining debris. Then, Complete Ultra protease inhibitors (EDTA-free; Roche) were added. Samples were stored at $-80^{\circ} \mathrm{C}$ prior to downstream analysis. Protein concentrations of $\mathrm{CM}$ were calculated using the Bradford assay with Coomassie Plus ${ }^{\mathrm{TM}}$ protein assay reagent (Thermo Scientific, Rockford, IL, USA), read at $660 \mathrm{~nm}$. In solution tryptic digestion was undertaken as previously described ${ }^{17}$. Liquid chromatography tandem mass spectrometry (LC-MS/MS) analysis was performed on trypsin digests using an Ultimate 3000 Nano system (Dionex, ThermoFisher Scientific, Waltham, MA, USA) on line to a Q-Exactive Quadrupole-Orbitrap instrument (Thermo Scientific) ${ }^{18}$. Proteins were identified using Peaks ${ }^{\circledR} 7$ PTM software (Version 7, Bioinformatics Solutions, Waterloo, Canada), searching against the UniMouse database, with a false discovery rate (FDR) of $1 \%$, a minimum of two unique peptides per protein and a confidence score $>50$.

\section{Label-free quantification of mass spectrometry proteomics data}

Progenesis QI $^{\mathrm{TM}}$ software (Version 4, Waters, Manchester, UK) was used to identify fold changes in protein abundance between scrambled siRNA duplex and viperin knockdown 
siRNA duplex and between the FLAG-empty and FLAG-viperin overexpression constructs as described previously ${ }^{19}$. Only unique peptides were used for quantification, and with $p$ values $<0.05$, were considered to be differentially expressed (DE).

\section{CXCL10 ELISA}

To determine CXCL10 protein concentrations in media, the mouse CXCL10/IP-10/CRG-2 DuoSet ELISA (R\&D \#DY466) and the human CXCL10/IP-10 Duoset ELISA (R\&D \#DY266) were utilized according to the manufacturer's protocol. Plates were colorimetrically measured on a ThermoScientific Multiskan FC plate reader (Waltham, MA, USA).

\section{Chondrogenic trans-differentiation of human dermal fibroblasts}

Human dermal fibroblasts (passage 7-9) from $3 \mathrm{CHH}$ patients (RMRP alleles of $\mathrm{CHH}$ patients carried the following mutations: $127 \mathrm{G}>\mathrm{A}$ and $261 \mathrm{C}>\mathrm{G} ; 4 \mathrm{C}>\mathrm{T}$ and $77 \mathrm{C}>\mathrm{T} ; 70 \mathrm{~A}>\mathrm{G}$ and $70 A>G)$ and 3 healthy controls were hyperconfluently plated $\left(100,000\right.$ cells $\left./ \mathrm{cm}^{2}\right)$ in aggrecan-coated (Sigma-Aldrich; $2.5 \mathrm{\mu g} / \mathrm{cm}^{2}$ ) wells as previously described ${ }^{20}$. Cells were directly plated in trans-differentiation medium which consisted of DMEM/F12+Glutamax (Invitrogen), 10\% FCS (PAA), 1\% antibiotic/antimycotic (Invitrogen), 1\% NEAA (Invitrogen) + 1\% ITS (Insulin, Transferrin, Selenium-Sodium Pyruvate; Life Technologies), $50 \mu \mathrm{g} / \mathrm{ml}$ ascorbic acid 2-phosphate (Sigma-Aldrich) and $1 \mathrm{ng} / \mathrm{ml}$ human recombinant TGF- $\beta 3$ (Life Technologies). Trans-differentiation medium was refreshed on day 2 and transdifferentiated cartilaginous nodules were harvested in TRIzol on day 3 of transdifferentiation for RNA isolation. Ethical permission was obtained from the medical ethical Institutional Review Board of Freiburg University Hospital and methods and experimental protocols to obtain dermal fibroblasts were carried out in accordance with the Freiburg University Hospital medical ethical Institutional Review Board, according to German law and abiding the Declaration of Helsinki principles. 


\section{Statistics}

Gene expression, ELISA, colorimetric and bioluminescence data were statistically analyzed using GraphPad Prism 5 (La Jolla, CA, USA). A two-tailed independent samples t-test was performed relative to the corresponding control condition. Individual values are presented in graphs in dot plots and the $p$-values and mean \pm SEM are indicated in the figures. The number of biological replicates and number of repeated experiments are indicated in the figure legends. Statistical significance was defined as $p<0.05$.

\section{Data availability}

The mass spectrometry proteomics data have been deposited to the ProteomeXchange Consortium (http://proteomecentral.proteomexchange.org) via the PRIDE partner repository ${ }^{21}$ with the dataset identifier PXD006999 and 10.6019/PXD006999. All other relevant data are available from the authors. 


\section{Results}

\section{Viperin protein is expressed throughout chondrocyte differentiation}

To investigate whether viperin is expressed during early chondrogenic differentiation, tissue sections were prepared from E15.5 mouse embryos. Expression of viperin was detected in growth plate chondrocytes throughout the developing growth plate, with highest expression levels in proliferating and terminally differentiating hypertrophic chondrocytes (Figure 1A). Cells in the upper zone of the growth plate displayed dispersed viperin positivity; viperin expression was either barely detectable in individual cells, was weakly expressed, or was relatively high expressed in individual cells (Figure 1B).

We further interrogated potential viperin expression during chondrogenic differentiation of the ATDC5 cell line $\mathrm{e}^{12,22}$ and of primary human bone marrow stem cells (hBMSCs) ${ }^{13}$. Chondrogenic differentiation of ATDC5 follows a well-defined cellular differentiation program with temporally increasing Sox9 and Col2a1 levels (Figure 2A/B) and increasing levels of Runx2 and Col10a1 (Figure 2C/D). During the first 3 days of ATDC5 chondrogenic differentiation viperin mRNA expression was hardly detectable (Figure 2E). From day 4 through day 14 (the latest time point tested), viperin mRNA expression was induced as compared to undifferentiated ATDC5 cells $(\mathrm{t}=0)$, and with a prominent peak expression at day 5 and 6 in differentiation. Similar expression dynamics were observed for viperin protein expression in these chondrogenic cultures (Figure 2F). During chondrogenic differentiation of hBMSCs (COL2A1 and COL10A1 mRNA expression in Figure 2G/H), peak induction of viperin mRNA expression was detected at day 7 of differentiation (Figure 2I), which is in agreement with viperin expression during ATDC5 chondrogenic differentiation. Collectively these data show that developing chondrocytes express viperin in vivo and in vitro; with undetectable to low expression in the early differentiation phase and a profoundly increased expression later in differentiation. 

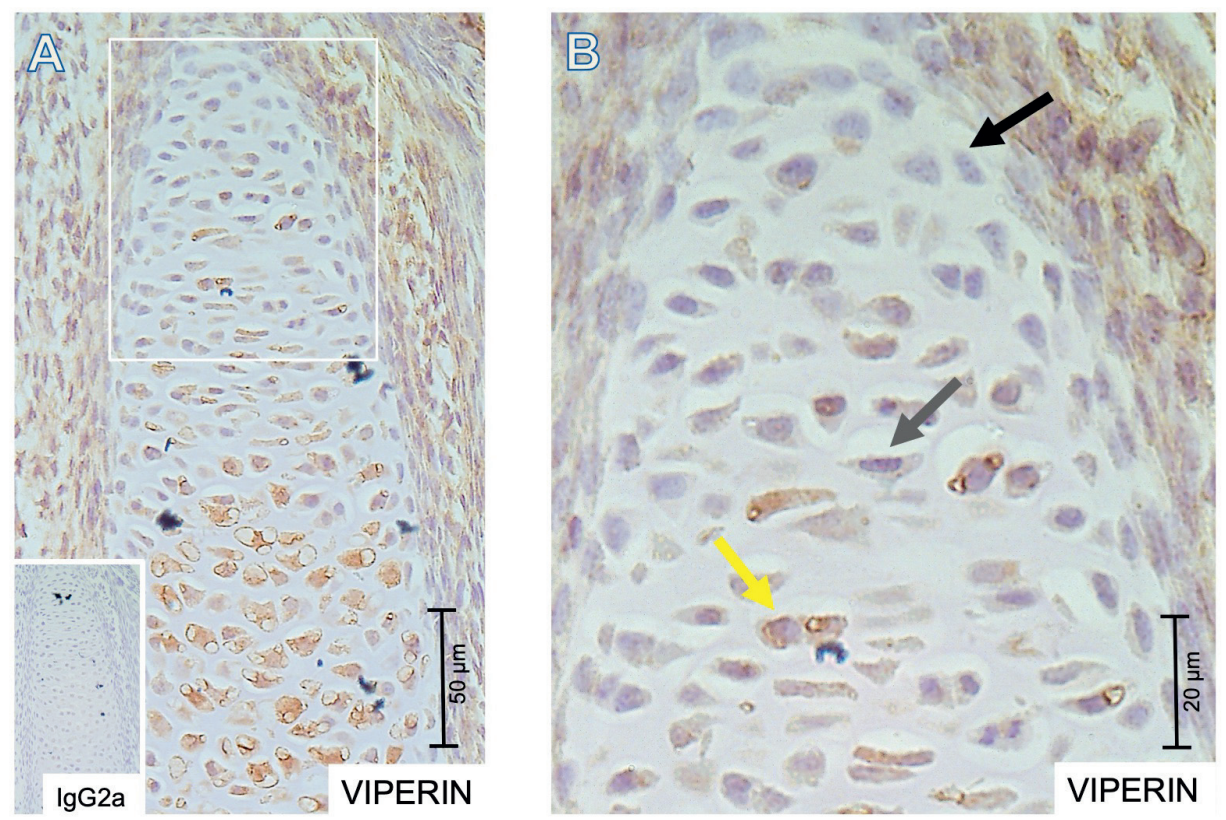

Figure 1. Viperin expression in the developing embryonal growth plate. Five micrometer-thick formalin-fixed paraffin-embedded tissue sections were prepared from E15.5 NMRI mouse embryos. Spatiotemporal expression of viperin was detected immunohistochemically. IgG2a was used as an isotype control. Overview of a representative immunohistochemically stained growth plate with IgG2a negative control in the insert (A). Indicated area from (A) enlarged. Black arrow indicates a representative cell with barely detectable viperin expression; grey arrow indicates a representative cell with weak viperin expression; yellow arrow indicates a representative cell with high viperin expression (B). Scale bars are indicated for magnification reference. 
A

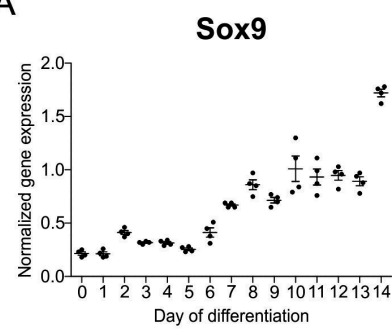

C

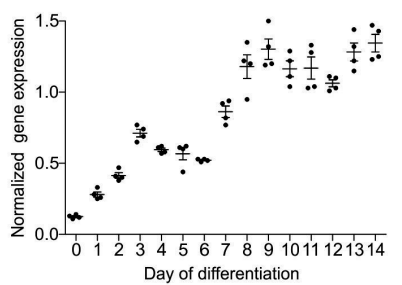

B

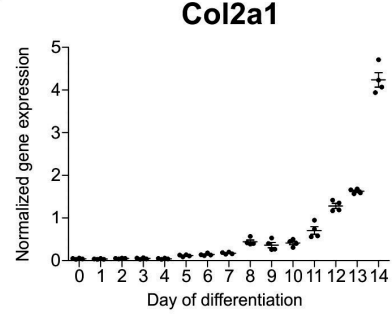

D

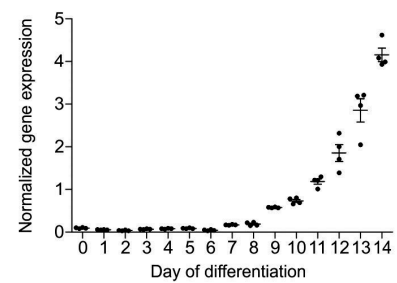

$\mathrm{F}$

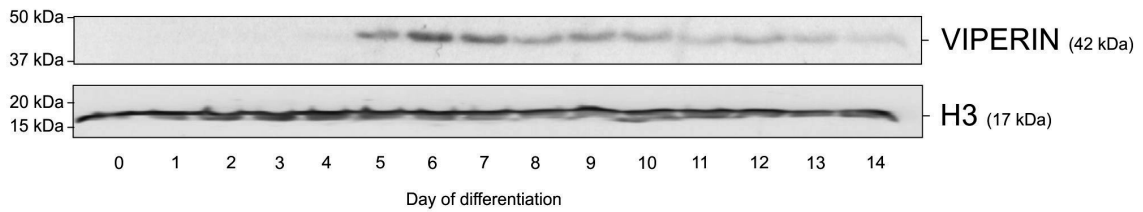

G
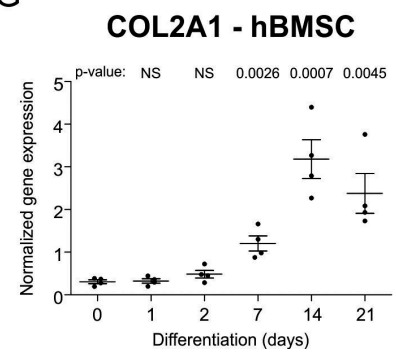

$\mathrm{H}$
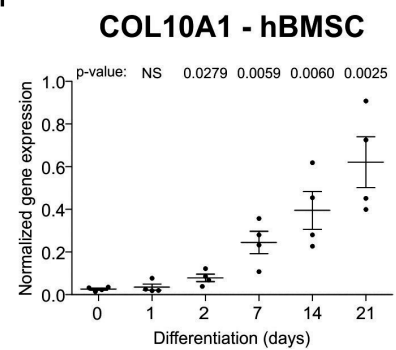

E

Viperin

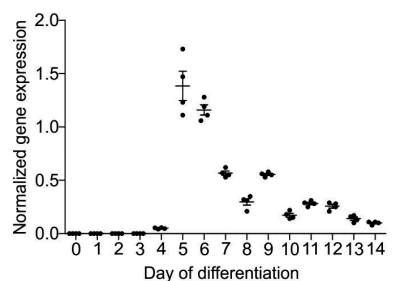




\section{Chapter 3}

marrow mesenchymal stem cells (hBMSCs) of 4 human subjects were differentiated in the chondrogenic lineage for $1,2,7,14$ and 21 days. Chondrogenic differentiation was confirmed by measuring COL2A1 and COL10A1 mRNA expression (G/H). In same samples expression of VIPERIN mRNA was measured (I). hBMSC gene expression data (G/H) was normalized to CYCLOPHILIN mRNA levels. Individual data points in dot plots represent the average value of 4 biological replicates of one hBMSC donor. The $p$-values are indicated and NS = not significant. Presented graphs are representative examples of three independent experiments. Error bars represent mean \pm SEM.

\section{Viperin knockdown reduces, while viperin overexpression increases protein secretion}

Previous work by Cresswell and colleagues ${ }^{23}$ showed that viperin regulates protein secretion from the ER and it has been suggested that this is one of the mechanisms by which viperin confers its cellular anti-viral activity. Since there is no other cell biological role identified for viperin so far that could explain its expression in differentiating chondrocytes, we postulated that viperin regulates protein secretion in differentiating chondrocytes. To investigate total protein secretion in relation to viperin levels we introduced a secretable Gaussia-luciferase (pGluc-CMV) at either day 3 or 5 in ATDC5 differentiation, followed by transfection of either a viperin siRNA duplex or a p3xFLAG-viperin plasmid at day 4 or 6 in differentiation, followed by sampling at day 5 or 7 , respectively. Reduced expression of viperin at day 5 or 7 in differentiation was demonstrated upon siRNA-mediated knockdown (Figure $3 \mathrm{~A}$ ) and ectopic expression of FLAG-viperin was confirmed at same time points (Figure 3A). Bioluminescent analyses showed that the amount of secreted Gaussia-luciferase was reduced following viperin knockdown and increased with viperin overexpression (Figure 3B). This was the case at both day 5 and 7 of differentiation. These data indicate that overall cellular protein secretion responds to changing viperin levels during chondrogenic differentiation. 
A

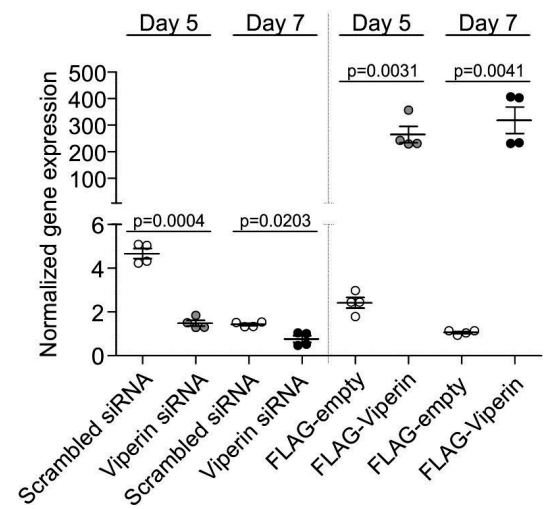

B

\section{Protein secretion}

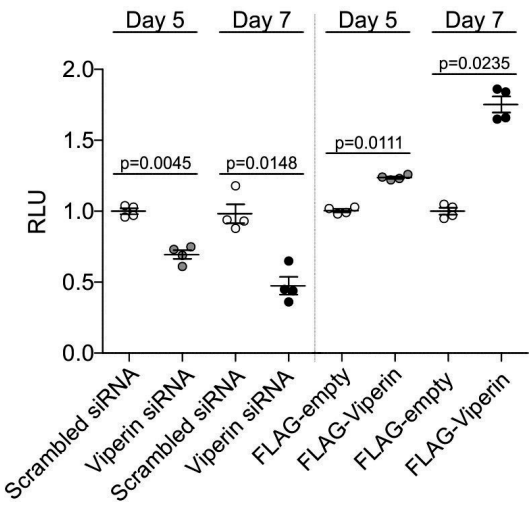

Figure 3. Viperin knockdown reduces, while viperin overexpression increases overall protein secretion. A CMV promoter-driven secreted Gaussia luciferase plasmid (pGluc-CMV) was co-transfected with a CMV promoter-driven firefly luciferase pGL4.20-CMV on day 3 or day 5 of ATDC5 chondrogenic differentiation. Then, on day 4 or 6 , viperin expression was either reduced by transfection of a viperin-specific siRNA duplex (Viperin siRNA) or a scrambled control siRNA duplex (Scrambled siRNA); or viperin expression was further induced by transfection of a p3xFLAG-viperin plasmid (FLAG-Viperin) or the empty p3xFLAG plasmid (FLAG-empty). Reduced or induced viperin expression (normalized to $\beta$-actin) was confirmed by RT-qPCR at day 5 or day 7 in differentiation (A) and individually presented in dot plots. Bioluminescence was assessed at day 5 or 7 in differentiation in culture supernatant (Gaussia luciferase) and cells (firefly luciferase). Gaussia bioluminescence was normalized to the firefly signal. Normalized relative light units (RLU) of controls were set at 1 and condition RLUs were calculated relative to the control RLUs and individually presented in dot plots (B). For statistical evaluation an independent samples t-test was performed relative to the corresponding controls using GraphPad Prism 5. Data are presented of 4 biological replicates. The pvalues are indicated and error bars indicate mean \pm SEM. Presented graphs are examples of 3 individual experiments. 


\section{The viperin-controlled secretome influences chondrogenic differentiation}

Considering the central role secreted signaling molecules play during chondrogenic differentiating ${ }^{6,24}$, we next questioned whether the observed viperin-controlled protein secretion plays a role in determining the chondrogenic outcome of the differentiation process via control over the chondrocyte's secretome. We investigated this possibility by reducing endogenous viperin levels or overexpressing viperin in differentiating ATDC5 chondrocytes and collecting conditioned media (CM) from these donor cultures. These $\mathrm{CM}$ were subsequently used to differentiate new ATDC5 cultures. Altered chondrogenic capacity of these cultures would reveal whether reduced or increased viperin levels during chondrogenic differentiation differentially influence the chondrocyte's secretome with downstream consequences for chondrogenic differentiation. Knockdown or over-expression of viperin was confirmed in the donor cultures from which CM were obtained (Figure 4A). Differentiation of ATDC5 cells in CM obtained from donor cultures in which viperin levels were reduced led to an overall increase in chondrogenic capacity, evidenced by increased expression of Sox9, Col2a1, Runx2, Col10a1 and Alpl (Figure 4B). Reciprocally, CM obtained from differentiating ATDC5 donor cultures in which viperin levels were over-expressed, displayed an inhibitory effect on the chondrogenic differentiation capacity, evidenced by reduced Sox9, Col2a1, Runx2, Col10a1 and Alpl expression (Figure 4C). The stimulatory and inhibitory actions of these $\mathrm{CM}$ on chondrogenic differentiation were further confirmed by determination of the glycosaminoglycan (GAG) content of these cultures. Differentiation of ATDC5 in CM obtained from donor cultures with reduced viperin levels caused an increased GAG content, while differentiation of ATDC5 in CM obtained from donor cultures in which viperin was over-expressed led to a reduced GAG content (Figure 4D). These results together indicate that alterations in viperin levels during chondrogenic differentiation change the cell's secretome with important functional consequences for differentiation. 
A

\section{Viperin}
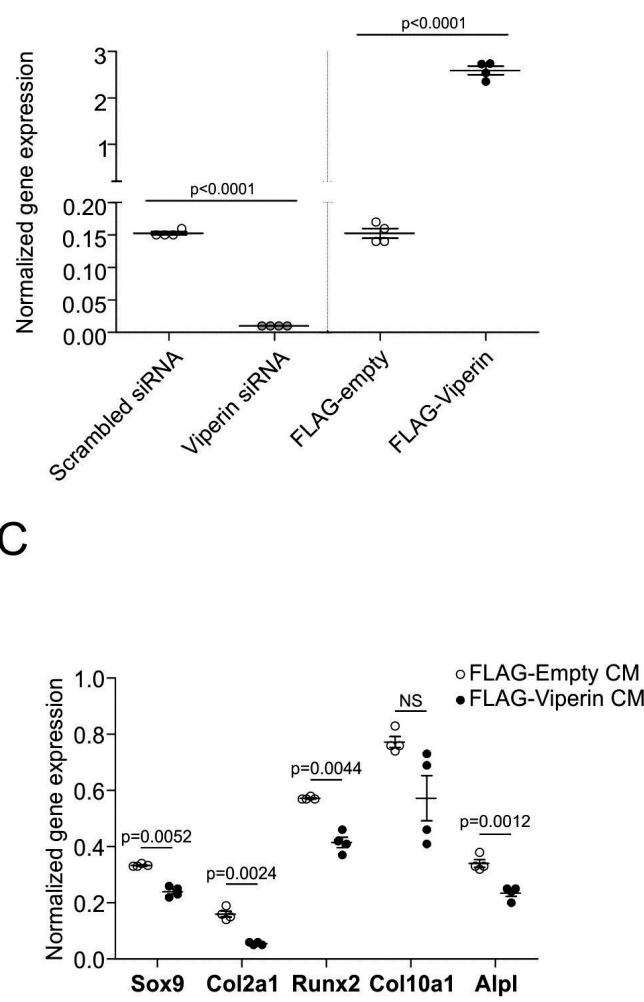

B

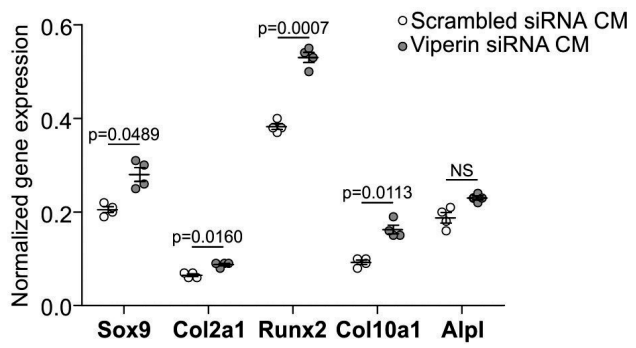

D

\section{Glycosaminoglycan content}

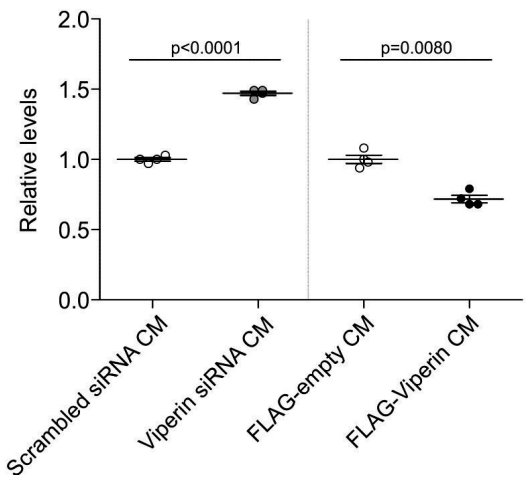

Figure 4. The viperin secretome influences chondrogenic differentiation. At day 5 in ATDC5 chondrogenic differentiation, viperin expression was either reduced by transfection of a viperin-specific siRNA duplex (Viperin siRNA) or a scrambled control siRNA duplex (Scrambled siRNA); or viperin expression was further induced by transfection of a p3xFLAG-viperin plasmid (FLAG-Viperin) or the empty p3xFLAG plasmid (FLAG-empty). Cells were then further differentiated until day 7 and viperin expression levels were determined (A). Conditioned culture supernatant (CM) from these donor cultures was harvested at day 7 and newly seeded ATDC5 cells were then differentiated with the CM supernatants for 7 days. RNA was isolated and Sox9, Col2a1, Runx2, Col10a1 and Alpl gene expression was determined in these samples (B/C). Glycosaminoglycan content (Alcian blue assay) was determined in ATDC5 cultures differentiated in CM supernatant from donor cultures in which viperin expression was reduced or further induced (D). RT-qPCR data was 
normalized to $\beta$-actin mRNA levels and individual normalized values are presented as dot plots. Glycosaminoglycan content data is presented as fold change relative to the corresponding control condition. For statistical evaluation an independent samples t-test was performed relative to the corresponding control condition using GraphPad Prism 5. The p-values are indicated and NS = not significant. All presented data were acquired from 4 biological replicates and error bars indicate mean \pm SEM. Graphs are representative examples of 3 individual experiments.

\section{Differential CXCL10 levels in viperin knockdown and overexpression secretomes}

Since media obtained from donor cultures with reduced or over-expressed viperin levels enhance or inhibit chondrogenic differentiation (respectively), we postulated that this was caused by a differential protein composition of their secretomes. To determine the differential proteome of the conditioned culture supernatants obtained from differentiating ATDC5 cultures with reduced or over-expressed viperin levels, we undertook a label-free mass-spectrometry proteomics approach using liquid chromatography tandem mass spectrometry (LC-MS/MS). When comparing the conditioned culture supernatants of differentiating ATDC5 cultures in which a scrambled siRNA was transfected or in which viperin levels were reduced using the viperin siRNA (Figure $5 \mathrm{~A}$ ), we identified six protein species that were differentially expressed in the CM (Figure $5 \mathrm{C}$ ). When comparing the conditioned culture supernatants of differentiating ATDC5 cultures in which an empty FLAGvector was transfected or in which viperin levels were over-expressed using the FLAGviperin plasmid (Figure 5B), we identified eight differentially expressed proteins in the $\mathrm{CM}$ (Figure 5C). Interestingly, CXCL10 (grey highlighted in Figure 5C) was the only protein species that was detected in both differential secretome proteomes. CXCL10 was decreased in $\mathrm{CM}$ obtained from cultures in which viperin levels were reduced and was increased in conditioned medium obtained from cultures in which viperin levels were over-expressed. Differential CXCL10 protein levels in these culture supernatants were independently confirmed by ELISA (Figure 5D). Since expression of both viperin and CXCL10 are interferondependent $^{1,25}$ (supplementary Figure 1), we next verified CXCL10 secretion dynamics during chondrogenic differentiation of ATDC5. Using an ELISA, supernatant samples from cultures in Figure 2 were analyzed for CXCL10 protein levels and we found that CXCL10 levels in 
culture supernatant (Figure 5E) during ATDC5 chondrogenic differentiation mirrored viperin expression dynamics (Figure 2E/F). Data together demonstrates that alterations in viperin levels during chondrogenic differentiation lead to specific differences in the protein composition of the chondrocyte's secretome, with CXCL10 a shared factor between the differential viperin-dependent secretomes.

A

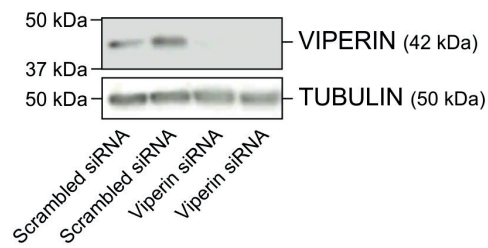

B

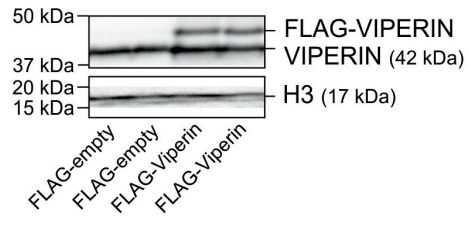

C

\begin{tabular}{|l|c|c|l|c|c|}
\hline \multicolumn{7}{|c|}{ Differentially secreted extracellular proteins } \\
\hline VIPERIN knockdown & FC & p-value & VIPERIN overexpression & FC & p-value \\
\hline $\begin{array}{l}\text { Platelet-derived growth factor a } \\
\text { (PDGFA) }\end{array}$ & -2.18 & 0.033 & Granulin (GRN) & 2.18 & 0.002 \\
\hline Collagen 6a3 (COL6A3) & -1.63 & 0.042 & $\begin{array}{l}\text { WNT1-inducible-signaling } \\
\text { pathway protein 2 (WISP2) }\end{array}$ & 2.57 & 0.002 \\
\hline Plasminogen (PLG) & -1.37 & 0.043 & $\begin{array}{l}\text { Insulin-like growth factor } \\
\text { binding protein 5 (IGFBP5) }\end{array}$ & -1.42 & 0.004 \\
\hline $\begin{array}{l}\text { SPARC-related modular calcium- } \\
\text { binding 1 (SMOC1) }\end{array}$ & 1.27 & 0.045 & $\begin{array}{l}\text { C-X-C motif chemokine 10 } \\
\text { (CXCL10) }\end{array}$ & 2.16 & 0.014 \\
\hline $\begin{array}{l}\text { C-C-C motif chemokine 10 } \\
\text { (CXCL10) }\end{array}$ & -1.44 & 0.048 & Collagen 6a1 (COL6A1) & -1.42 & 0.017 \\
\hline $\begin{array}{l}\text { Insulin-like growth factor binding } \\
\text { protein 4 (IGFBP4) }\end{array}$ & -1.52 & 0.048 & Collagen 12a1 (COL12A1) & -1.32 & 0.025 \\
\hline & & & Fibrillin-2 (FBN2) & -2.37 & 0.029 \\
\hline & & & C-C motif chemokine 2 (CCL2) & 1.53 & 0.032 \\
\hline \hline
\end{tabular}

Figure 5A-C. The viperin secretome and CXCL10. At day 5 in ATDC5 chondrogenic differentiation, viperin expression was either reduced by transfection of a viperin-specific siRNA duplex (Viperin siRNA) or a scrambled control siRNA duplex (Scrambled siRNA); or viperin expression was further induced by transfection of a p3xFLAG-viperin plasmid (FLAG-Viperin) or the empty p3xFLAG plasmid (FLAG-empty). Cells were then further differentiated until day 7 and viperin expression levels were determined (A/B). Immunoblots are shown for two independent representative examples. Culture supernatants from these day-7 cultures were collected and the 
protein secretome was determined by label-free LC-MS/MS. Differentially secreted extracellular protein species (control versus condition) with $p<0.05$ are shown (C).

D

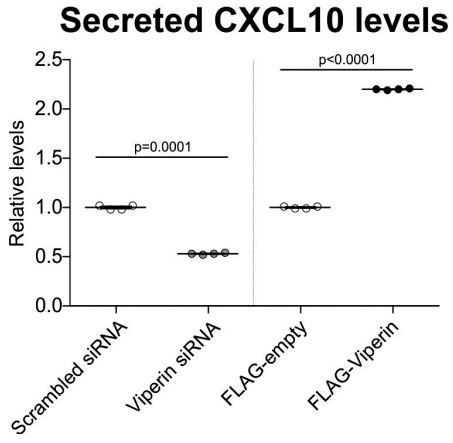

E

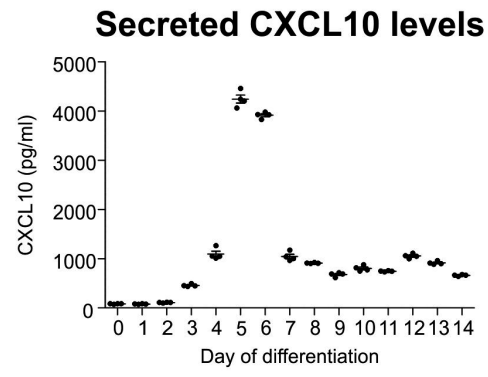

Figure 5D-E. The viperin secretome and CXCL10. At day 5 in ATDC5 chondrogenic differentiation, viperin expression was either reduced by transfection of a viperin-specific siRNA duplex (Viperin siRNA) or a scrambled control siRNA duplex (Scrambled siRNA); or viperin expression was further induced by transfection of a p3xFLAG-viperin plasmid (FLAG-Viperin) or the empty p3xFLAG plasmid (FLAG-empty). In culture supernatants analyzed with LC-MS/MS, secreted CXCL10 levels were determined (D). In ATDC5 culture supernatants from Figure 2, secreted CXCL10 levels were determined $(E)$. The $p$-values are indicated and error bars indicate mean \pm SEM.

A

Secreted CXCL10 levels

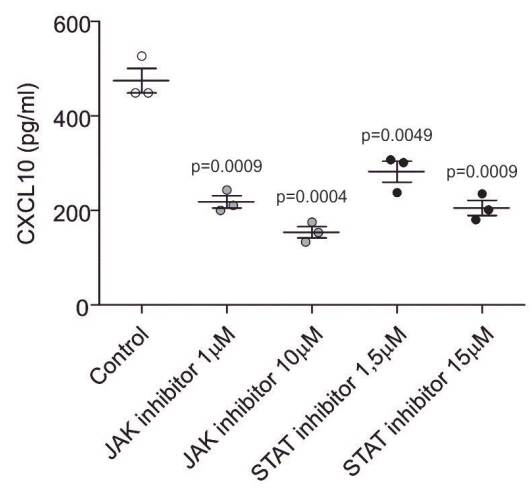

B

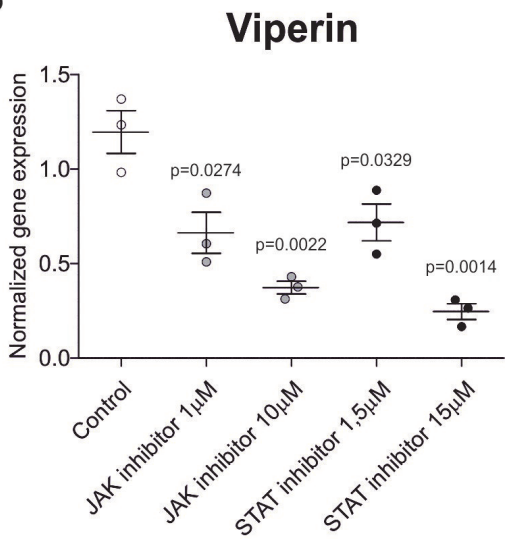

Supplementary Figure 1. Inhibition of interferon signaling activity during ATDC5 chondrogenic differentiation inhibits secreted CXCL10 levels in culture supernatant 
and attenuates viperin expression. ATDC5 cells were differentiated into the chondrogenic lineage until day 6. Downstream interferon signaling activity was then inhibited for 24 hours until day 7. The JAK inhibitor Ruxolitinib was used at $1 \mu \mathrm{M}$ or $10 \mu \mathrm{M}$. The STAT inhibitor Fludarabine was used at $1.5 \mu \mathrm{M}$ or $15 \mu \mathrm{M}$. Control was treatment with vehicle. At day 7 culture supernatant was collected and cells were harvested for total RNA isolation, followed by CDNA synthesis. In culture supernatants secreted CXCL10 protein levels were determined (A). Expression of viperin was determined by RT-qPCR Sox9 (B). Secreted CXCL10 data are absolute concentrations (pg/mL) and presented in dot plots. RT-qPCR data was normalized to $\beta$-actin mRNA levels and individual normalized values are presented in dot plots. All data were acquired from 3 biological replicates. An independent samples t-test was performed relative to control using GraphPad Prism 5. The p-values are indicated and error bars represent mean \pm SEM.

\section{CXCL10 inhibits chondrogenic differentiation}

Conditioned culture medium obtained from differentiating ATDC5 cultures in which viperin levels were over-expressed was found to inhibit chondrogenic differentiation. Besides a limited number of other DE proteins, CXCL10 was the only shared protein species that was differentially present in the viperin-dependent secretomes and was increased in viperin over-expression culture supernatant. We therefore postulated that CXCL10 contributes to the inhibitory action on chondrogenic differentiation of the viperin over-expression culture supernatant (Figure 4C). To determine whether CXCL10 inhibits chondrogenic differentiation, differentiating ATDC5 cultures were exposed to exogenously added mCXCL10 from day 5 of differentiation until day 7 of differentiation (the main time frame in which we observed peak expression of viperin and peak CXCL10 concentrations; Figure 2E/F and Figure 5E). Analysis showed that exogenously added mCXCL10 attenuated chondrogenic differentiation, evidenced by decreased expression of Sox9, Col2a1, Runx2, Col10a1 and Alpl (Figure 6A). To verify the inhibitory action of CXCL10 in an independent model for chondrogenic differentiation, primary hBMSCs were differentiated into the chondrogenic lineage and exposed to increasing concentrations hCXCL10 from day 7 (peak viperin expression in hBMSC chondrogenic differentiation; Figure 2l) in chondrogenic differentiation onward. CXCL10 inhibited chondrogenic differentiation of hBMSCs, evidenced by a decline of COL2A1 and COL10A1 mRNA expression (Figure 6B). These data indicate that viperin- 


\section{Chapter 3}

dependent alterations in secreted CXCL10 levels influence chondrogenic differentiation capacity.
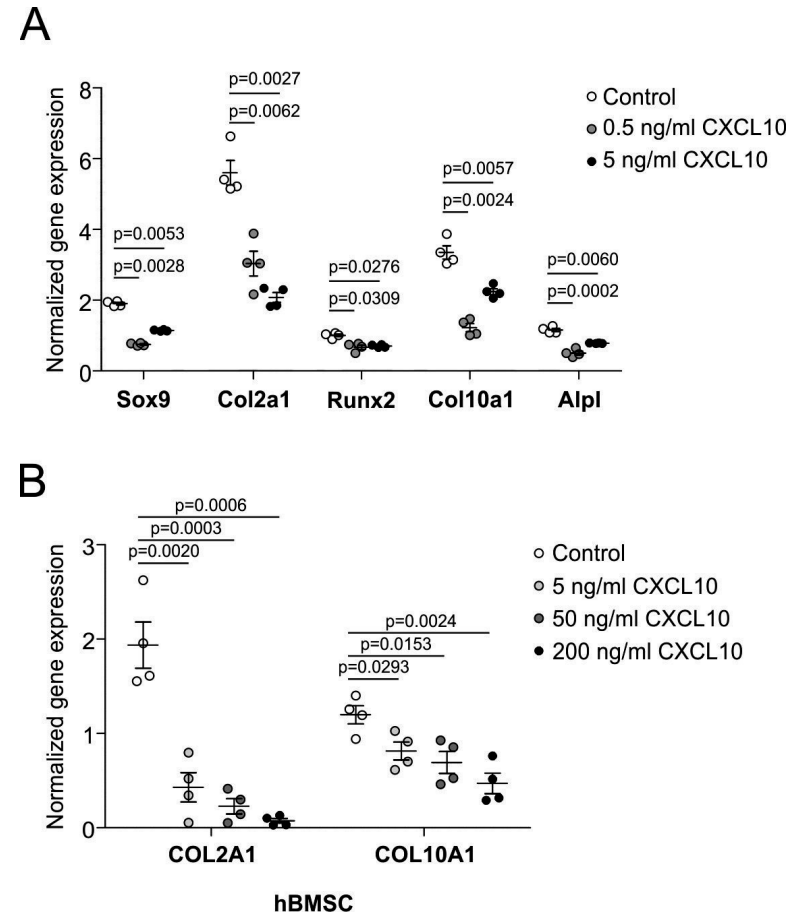

Figure 6. CXCL10 attenuates chondrogenic differentiation. ATDC5 cells were differentiated and exposed to mCXCL10 from day 5 until day 7. RNA was isolated and expression of Sox9, Col2a1, Runx2, Col10a1 and Alpl mRNAs was determined (A). Human bone marrow mesenchymal stem cells (hBMSCs) were differentiated in the chondrogenic lineage for 7 days and exposed to hCXCL10 until day 21. RNA was isolated and expression of COL2A1 and COL1OA1 mRNAs was determined (B). ATDC5 data were acquired from 4 biological replicates, normalized to $\beta$-actin mRNA levels and individual normalized values are presented in dot plots. hBMSC gene expression data was normalized to CYCLOPHILIN mRNA levels. Individual data points in dot plots represent the average value of 4 biological replicates of one hBMSC donor. For statistical evaluation an independent samples t-test was performed relative to the corresponding controls using GraphPad Prism 5. The pvalues are indicated and error bars show mean \pm SEM. The graphs are a representative example of 3 individual experiments. 


\section{Viperin levels and CXCL10 change SMAD2/3-dependent TGF- $\beta$ activity during chondrogenic differentiation of ATDC5}

The chondro-promotive and -inhibitory actions of $\mathrm{CM}$ obtained from donor cultures in which viperin expression was altered, as well as the chondro-inhibitory action of CXCL10, leads to an overall inhibited or increased expression of chondrogenic as well as hypertrophic marker genes. This indicates that the activity of a basic molecular pathway responsible for chondrogenic differentiation is altered and we therefore postulated the involvement of the major chondrogenic TGF- $\beta / S M A D 2 / 3$ pathway. Viperin levels were either reduced or overexpressed in differentiating ATDC5 cultures from day 5 to day 7 (Figure 7A), and CM were collected. Subsequently, proliferating ATDC5 cells were used as a bioassay for SMAD2/3dependent TGF- $\beta$ activity by transfection of a CAGA12-luciferase reporter plasmid ${ }^{15,16}$. This TGF- $\beta /$ SMAD2/3 reporter bioassay was then exposed to the above CM to determine their TGF- $\beta / S M A D 2 / 3$-activity modulating action. We found that the $\mathrm{CM}$ obtained from differentiating ATDC5 cultures in which viperin levels were reduced displayed a TGFB/SMAD2/3-activity promoting action (Figure 7B). In contrast, CM obtained from differentiating ATDC5 cultures in which viperin levels were over-expressed displayed a TGF$\beta / S M A D 2 / 3$-activity attenuating action (Figure $7 B$ ). In concert with these findings Pai1 and SMAD7 mRNA levels (TGF- $\beta /$ SMAD2/3 target genes ${ }^{15,26}$ ) were increased or decreased when ATDC5 was differentiated in CM (same as utilized in experiments shown in Figure 4B/C) from viperin knock-down or over-expression cultures, respectively (Figure 7C/D). Subsequently, we determined whether differential CXCL10 levels directly alter TGF$\beta / S M A D 2 / 3$ activity during chondrogenic differentiation. ATDC5 cells were transfected with the CAGA12-luciferase reporter plasmid at day 5 in chondrogenic differentiation. Then, starting at day 6, cells were exposed to increasing concentrations recombinant murine CXCL10 and sampled for bioluminescent analyses 24 hours later. Data show that CXCL10 dose-dependently reduces TGF- $\beta /$ SMAD2/3 reporter activity in these cultures (Figure 7E). Immunoblotting for SMAD2 phosphorylation (pSMAD2C) further showed that CXCL10 inhibits SMAD2 phosphorylation during ATDC5 chondrogenic differentiation (Figure 7F), which is in concert with the observed reduction of TGF- $\beta / S M A D 2 / 3$ reporter activity. Together these results demonstrate that alterations in viperin expression levels and CXCL10 impact SMAD2/3-dependent TGF- $\beta$ activity during chondrogenic differentiation. This is in 
concert with the observed impact of these conditions on chondrogenic differentiation capacity (Figure 4B/C/D and Figure 6).

A

Viperin

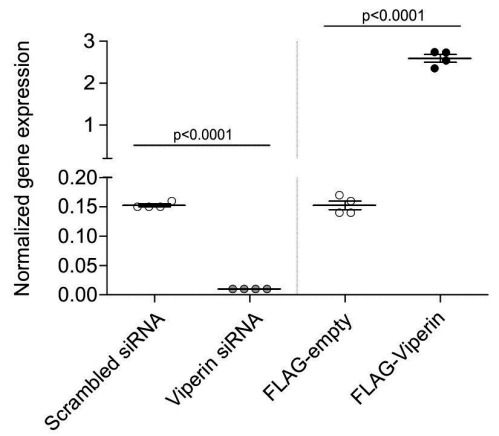

C

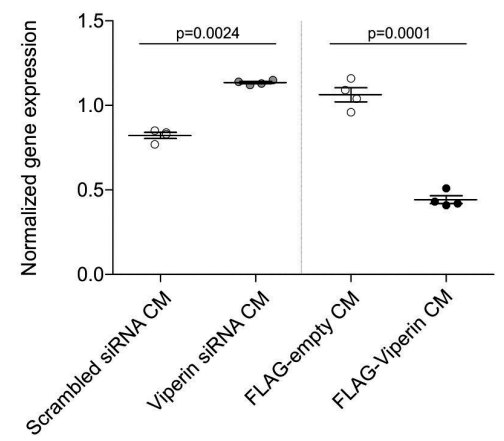

E

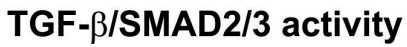

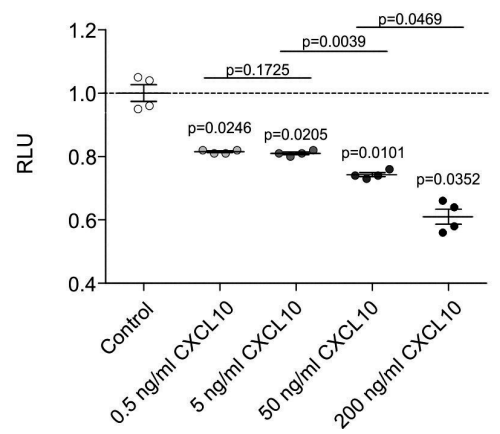

B TGF- $\beta /$ SMAD2/3 activity

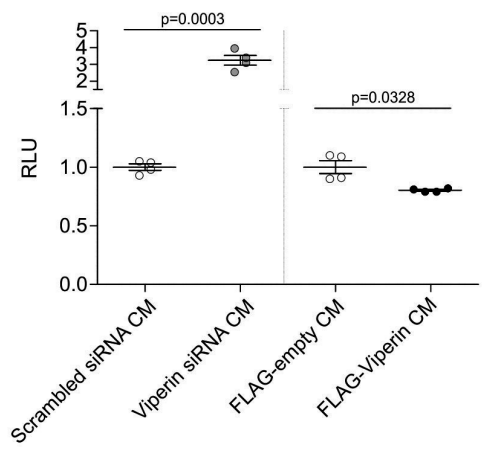

D

\section{Smad7}

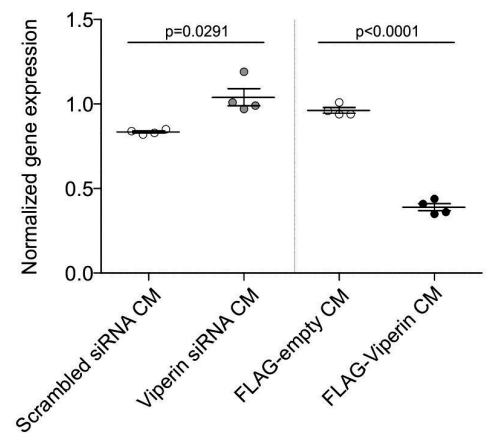

$\mathrm{F}$

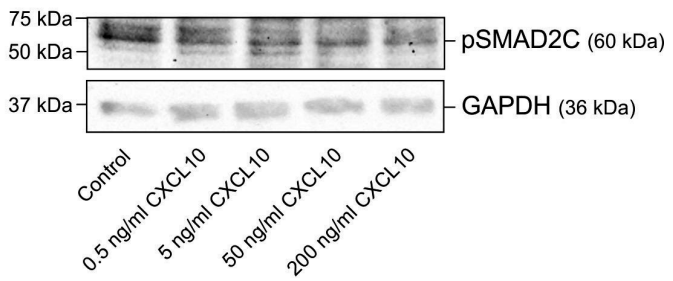


Figure 7. TGF- $\beta /$ SMAD2/3 activity is controlled by the viperin secretome and CXCL10. At day 5 in ATDC5 chondrogenic differentiation, viperin expression was either reduced by transfection of a viperin-specific siRNA duplex (Viperin siRNA) or a scrambled control siRNA duplex (Scrambled siRNA); or viperin expression was further induced by transfection of a p3xFLAG-viperin plasmid (FLAG-Viperin) or the empty p3xFLAG plasmid (FLAG-empty). Cells were then further differentiated until day 7 and viperin expression levels were determined (A). Conditioned culture supernatants (CM) from these day-7 cultures were collected. Proliferating ATDC5 were used as a TGF- $\beta /$ SMAD2/3 bioassay by co-transfecting a CAGA-12 firefly luciferase TGF- $\beta /$ SMAD3 reporter and pGluc-CMV plasmid. The TGF- $\beta /$ SMAD2/3 bioassay was then exposed to the CM supernatants for 24 hours and supernatant and cells were collected for bioluminescence analyses. Firefly bioluminescence was normalized to the Gaussia signal (B). Gene expression of downstream TGF- $\beta$ target genes Pai1 and Smad7 was determined on samples from Figure 4B/C (C/D). ATDC5 cells were differentiated and co-transfected with a CAGA-12 firefly luciferase TGF- $\beta /$ SMAD3 reporter and pGluc-CMV plasmid on day 5 in chondrogenic differentiation. At day 6 in differentiation cells were exposed to $0.5,5,50$ or $200 \mathrm{ng} / \mathrm{ml}$ recombinant mouse CXCL10 for 24 hours until day 7. Culture supernatant and cells were collected for bioluminescence analyses. Firefly bioluminescence was normalized to the Gaussia signal (E). ATDC5 cells were differentiated until day 5 and then exposed to $0.5,5,50$ or $200 \mathrm{ng} / \mathrm{ml}$ mouse CXCL10 until day 7. Protein extracts were separated by SDS-PAGE and electro-blotted on membranes, followed by PSMAD2C immuno detection. GAPDH was used as a loading control (F). All quantitative data were acquired from 4 biological replicates. RT-qPCR data were normalized to $\beta$-actin mRNA levels and individual normalized values are presented in dot plots. Bioluminescence data are presented as normalized RLUs. RLUs of controls were set at 1 and RLUs of conditions were calculated relative to the control RLUs. For statistical evaluation an independent samples t-test was performed relative to the corresponding controls using GraphPad Prism 5. The pvalues are indicated and error bars represent mean \pm SEM. Graphs are representative examples of 3 individual experiments. 


\section{Impaired chondrogenic trans-differentiation of fibroblasts from $\mathrm{CHH}$ patients is associated with increased viperin and CXCL10 levels}

Mutations in the RMRP gene, encoding the essential RMRP snoRNA present in the RNase MRP macromolecular protein-RNA complex ${ }^{4}$, are the cause of cartilage-hair hypoplasia-type human skeletal dysplasias ${ }^{3,5}$. Viperin mRNA is a substrate for endoribonucleolytic degradation by RNase $\mathrm{MRP}^{2}$. Taking into account the here identified chondrogenic regulatory role of a viperin-CXCL10-TGF- $\beta /$ SMAD2/3 axis, we postulated that a similar mechanism is active during the impaired chondrogenic trans-differentiation that we recently discovered in $\mathrm{CHH}$ fibroblasts ${ }^{11}$. In ATDC5, reduction of RMRP snoRNA levels by transfection of an RMRP-specific siRNA (Figure 8A) indeed led to the induction of viperin expression ( ${ }^{11}$ and Figure $8 \mathrm{~B}$ ), recapitulating the endoribonucleolytic relationship between RNase MRP and its viperin mRNA substrate. A trans-differentiation protocol was used that drives dermal fibroblasts toward a chondrocyte-like phenotype ${ }^{20}$. As described before ${ }^{11}$, RMRP expression and chondrocytic trans-differentiation of $\mathrm{CHH}$ fibroblasts was impaired, as evidenced by inhibited expression of RMRP, SOX9, COL2A1, RUNX2, COL10A1 and ALPL (Figure 8C). In concert with a $\mathrm{CHH}$-associated pathological defective RNase MRP endoribonucleolytic activity, we found that viperin expression in chondrogenic trans-differentiated $\mathrm{CHH}$ fibroblasts was increased (Figure 8D). This was accompanied with increased levels of secreted CXCL10 protein in these culture supernatants (Figure $8 \mathrm{E}$ ) and decreased expression of the TGF- $\beta$ target genes PAI1 and SMAD7 (Figure 8F). These data suggest that defective chondrogenic trans-differentiation of $\mathrm{CHH}$ fibroblasts is caused by changes in TGF- $\beta$ activity, induced by alterations in the viperin-CXCL10-TGF- $\beta /$ SMAD2/3 axis. 
A

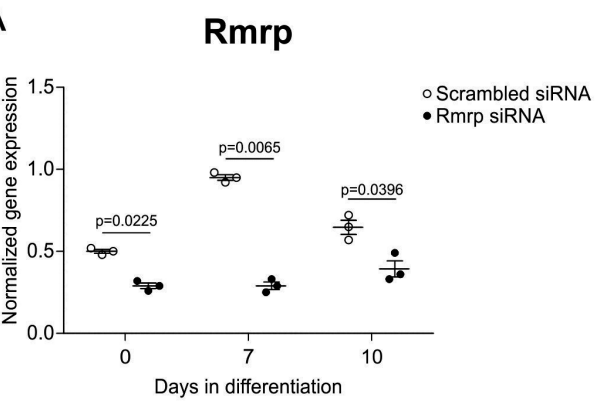

C

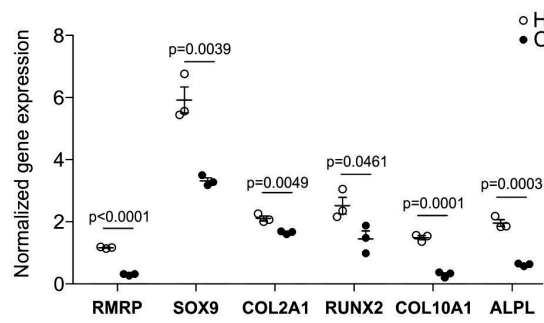

E

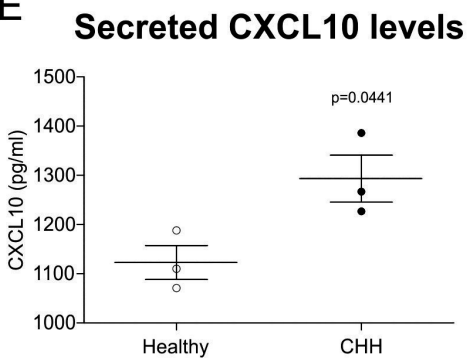
Healthy
B

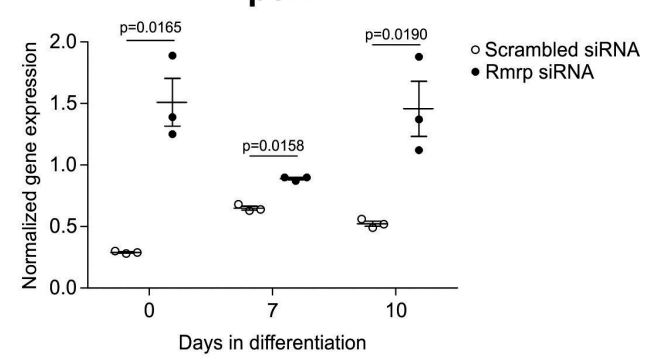

$\mathrm{D}$ VIPERIN

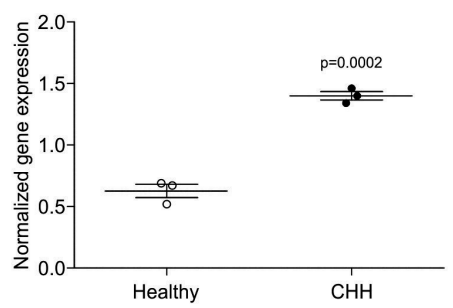

$\mathrm{F}$

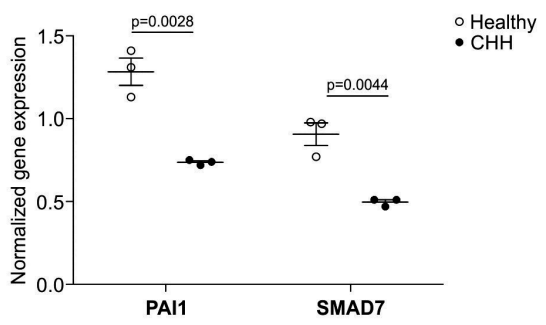

Figure 8. The viperin-CXCL10-TGF- $\beta / S M A D 2 / 3$ axis is deregulated in chondrocytic CHH cells. Rmrp RNA expression was reduced in ATDC5 cells by transfection of a specific siRNA duplex on day -1 , day 2 , and day 5 during chondrogenic differentiation. A scrambled siRNA was used as control. Samples were harvested for RT-qPCR analysis at day 0,7 or 10 in differentiation. Expression of Rmrp (A) and viperin (B) was determined at indicated time points. Data was normalized to $\beta$-actin mRNA levels and individual normalized values are presented in dot plots. Data were acquired from 3 biological replicates. An independent samples t-test was performed relative to scrambled control using GraphPad Prism 5. The p-values are indicated and error bars represent mean \pm SEM. Human dermal fibroblasts from three $\mathrm{CHH}$ patients (RMRP alleles of $\mathrm{CHH}$ patients carried following mutations: $127 \mathrm{G}>\mathrm{A}$ and $261 \mathrm{C}>\mathrm{G} ; 4 \mathrm{C}>\mathrm{T}$ and $77 \mathrm{C}>\mathrm{T} ; 70 \mathrm{~A}>\mathrm{G}$ and $70 \mathrm{~A}>\mathrm{G}$ ) and three healthy controls were trans-differentiated into the chondrogenic lineage by hyperconfluent plating in wells coated with Aggrecan ${ }^{11,20}$. RNA was isolated at day 3 of trans-differentiation and gene 
expression of RMRP, SOX9, COL2A1, RUNX2, COL10A1 and ALPL mRNAs was determined (C). Gene expression of VIPERIN (D) and of PAI1 and SMAD7 (F) was determined in samples from (C). Supernatants were collected from these cultures and secreted CXCL10 protein was determined with ELISA (E). Gene expression data from trans-differentiated fibroblasts was normalized to CYCLOPHILIN mRNA levels and individual normalized values are presented in dot plots. Secreted CXCL10 data are absolute concentrations $(\mathrm{pg} / \mathrm{mL}$ ) and presented in dot plots. For statistical evaluation independent samples t-tests were performed relative to healthy controls using GraphPad Prism 5. The p-values are indicated. Error bars are mean \pm SEM. Graphs are representative examples of three independent experiments.

\section{Discussion}

Viperin expression was detectable early in ATDC5 and hBMSC chondrogenic differentiation and was highly induced at day 5-6-7 of differentiation. In the majority of embryonal growth plate resting zone progenitor cells viperin expression was weak, representing the first days of in vitro chondrogenic differentiation. Interestingly, a sub-population of resting zone cells displayed high levels of viperin expression. Chondrogenic differentiation is synchronously initiated in cell culture, while this is not expected to be synchronous in the growth plate's resting zone. We speculate that high viperin-expressing cells in the resting zone are a representation of asynchronous initiation of chondrogenic differentiation and that these cells are in a similar differentiation phase as ATDC5 and hBMSC cells during their early differentiation, when peak expression of viperin was observed. At later stages in chondrogenic differentiation viperin expression remained increased. This was also observed in growth plates, where proliferating and pre-hypertrophic chondrocytes are positive for viperin expression. Viperin expression is remarkably high in terminally differentiating growth plate chondrocytes. This was not observed in late ATDC5 and hBMSC differentiation time points tested. ATDC5 and hBMSCs are excellent in vitro models for chondrogenic differentiation ${ }^{22}$. However these models do not enter apoptosis to fully terminally differentiate, as hypertrophic chondrocytes in the growth plate do. The spatiotemporal orchestration of cell differentiation in growth plates and the lack of such spatiotemporal cues in vitro may lead to the absence of this differentiation phase in in vitro chondrogenesis 
models and explain the observed difference in viperin expression compared to growth plates.

Viperin was originally discovered as an interferon-inducible protein, suggesting that induction of viperin expression during chondrogenic differentiation may be driven by an intrinsic interferon-related signaling activity (further supported by data in supplementary Figure 1). Interferon-related signaling during chondrogenic differentiation is almost unexplored. Interferon-inducible proteins $\mathrm{p} 202^{27}, \mathrm{p} 204^{28}$ and $\mathrm{PKR}^{29}$ have been described to be important in chondrogenic differentiation. Inhibition of JAK rescues chondrogenic differentiation in osteoarthritis-like conditions ${ }^{30}$ and IFN- $\gamma$ has been demonstrated to inhibit transcription of the COL2A1 gene ${ }^{31,32}$ in mature chondrocytes. STAT1 expression was previously found specifically induced at day 7 in ATDC5 chondrogenic differentiation ${ }^{29}$, supporting an intrinsically activated interferon signaling response as explanation for viperin induction at day 5-6-7. Different potential mechanisms to explain viperin's antiviral actions include an Fe-S cluster-dependent virus inhibitory action on lipid droplets ${ }^{23,33}$, the inhibition of farnesyl diphosphate synthase leading to important changes in plasma membrane fluidity ${ }^{34}$, the induction of a crystalloid $E R^{23}$, and the inhibition of protein secretion from the $\mathrm{ER}^{23}$. Viperin has been shown to recruit IRAK1 and TRAF6 to lipid droplets in pDCs, leading to nuclear translocation of IRF7 and the production of type I interferons ${ }^{35}$. These actions are specifically activated in response to viral infection and it is therefore surprising to find that in a non-viral context viperin is active as a regulator of cellular differentiation. Previously, alterations in viperin expression have been detected during adipogenic differentiation ${ }^{36}$ and podocyte differentiation ${ }^{37}$. Middle zone articular cartilage chondrocytes express higher amounts of viperin as compared to superficial zone chondrocytes ${ }^{38}$ and viperin expression has been described in osteocytes ${ }^{39}$. It is therefore likely that the consequences of viperin expression are not limited to differentiating chondrocytes. Until now it remained unexplored whether alterations in viperin levels could influence cellular differentiation processes. Following the findings by Cresswell and colleagues that viperin overexpression inhibits protein secretion from the ER in HepG2 and 293T cells ${ }^{23}$, we examined whether this was also the case during chondrogenic differentiation. We found that also in chondrogenic differentiation viperin influences the rate of protein secretion, however in the opposite direction as compared to the findings reported by the Cresswell group. We speculate that due to different cell lineages, differentiation stages and the non-viral context, important 
differences occur in the viperin interactome ${ }^{35}$ that change the manner by which viperin regulates protein secretion from the ER.

We observed that total protein secretion alters in response to viperin levels, and until now no specificity has been reported for viperin-regulated protein secretion. Therefore we expect that the protein species that we found differentially secreted following manipulation of viperin levels are either relatively unstable and inhibition of cellular protein secretion would thus cause a rapid decrease in the level of this species within the secretome, or are secreted in low abundance and increased protein secretion would rapidly lead to detectable differences in the level of this protein species in the secretome. In line with a viperinmediated intrinsic interferon response during chondrogenic differentiation, the CXCL10 we detected in our LC-MS/MS analyses is an interferon-inducible chemokine (IP-10). Interestingly, platelet-derived growth factor subunit A (PDGFA) was also found differentially expressed in the viperin over-expression secretome and PDGF has been described to synergistically act with IFN- $\gamma$ to induce CXCL10 expression in blood-derived macrophages ${ }^{25}$. CXCL10 has previously been described to be expressed during ATDC5 chondrogenic differentiation ${ }^{40}$, as well as during hBMSC chondrogenic differentiation ${ }^{41}$. Our data demonstrate that CXCL10 inhibits chondrogenic differentiation and TGF- $\beta$ signaling, which is fully in line with the inhibitory action of viperin-overexpression $\mathrm{CM}$ on chondrogenic differentiation.

Since we utilized conditioned media as a means to study the secretomic consequences of alterations in viperin expression during chondrogenic differentiation, we cannot distinguish paracrine from autocrine secreted signals. However, it is currently not clear why chondrogenic differentiation is regulated via viperin. Control over the activity of differentiation signals in the early differentiation phase may be required to enable the cell to undertake chromatin remodeling and coordinated transcriptomic reprogramming, before it can adopt a fully differentiated phenotype. We expect that temporal para- and/or autocrine viperin-dependent $\mathrm{CXCL10}$ secretion may aid in this by antagonizing TGF- $\beta$ signaling and pacing cellular differentiation. Binding of CXCL10 with its CXCR3 receptor is known to activate PI3K in human airway epithelial cells ${ }^{42}$ and recently, PI3K activity has been shown to be involved in attenuation of SMAD2/3 activity ${ }^{43}$. Indeed we observed that CXCL10 inhibits phosphorylation of SMAD2, providing a potential mechanistic link between viperindependent CXCL10 secretion and TGF- $\beta$-driven chondrogenic differentiation. Alternatively, 
crosstalk has been identified between IFN- $\gamma$ and TGF- $\beta$ signaling in which IFN- $\gamma$-dependent STAT1 activity antagonizes SMAD3-dependent TGF- $\beta$ signaling ${ }^{44}$.

With a number of varying RNase MRP substrate RNAs it would not be likely that all aspects of $\mathrm{CHH}$ pathobiology are caused by defective processing of one specific substrate. Instead, defective processing of one or the other specific RNase MRP substrate RNA will have different implications for different tissue/cell types. CHH-related defective processing of viperin mRNA leads to aberrant viperin levels in chondrocytic cells as shown in this study. Viperin responses ${ }^{45}$ and $\mathrm{CXCL} 0^{46}$ have been implicated in T cell function and in a microarray mRNA profiling it was found that viperin was one of the highest differentially expressed genes upregulated in $\mathrm{CHH}$ leukocytes ${ }^{10}$, together with an enrichment of other interferonrelated genes. Since $\mathrm{CHH}$ also presents with defective immunity caused by T-cell deficiency ${ }^{47}$ and together with our findings that viperin and CXCL10 regulate chondrogenic differentiation, is thus tempting to speculate that interferon-related signaling through viperin, and via CXCL10, is an important aspect of the molecular mechanism leading to growth plate and T-cell defects observed in $\mathrm{CHH}$.

In conclusion our data link the antiviral protein viperin to chondrogenic development via a viperin-CXCL10-TGF- $\beta /$ SMAD2/3 axis (Figure 9), and show that a similar molecular mechanism is deregulated in $\mathrm{CHH}$ chondrocytic cells. For the first time we identified a molecular route that may clarify impaired chondrogenic differentiation of $\mathrm{CHH}$ patient cells.

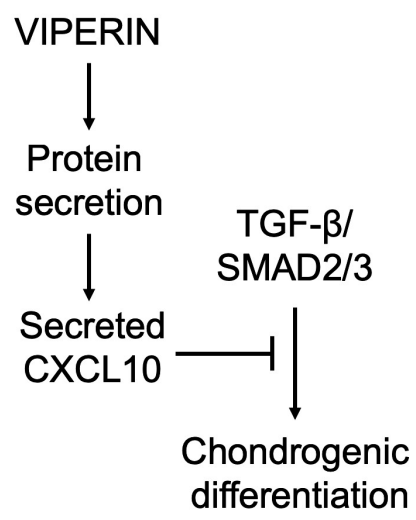


Figure 9. Model of the interactions between viperin and chondrogenic differentiation. A schematic model of the interactions between viperin and chondrogenic differentiation, suggested by our results. Viperin regulates protein secretion and controls the level of secreted CXCL10. CXCL10 inhibits TGF- $\beta /$ SMAD2/3 activity, which in turn controls the level of chondrogenic differentiation.

\section{Acknowledgements}

The authors thank Prof. P. van der Kraan (Experimental Rheumatology, Radboud University Medical Center, Nijmegen, the Netherlands) for PAI1 and SMAD7 primer sequences; Prof. G. Pruijn (Biomolecular Chemistry, Radboud University, Nijmegen, the Netherlands) for discussion; Dr. T. Dalmay (School of Biological Sciences, University of East Anglia, United Kingdom) for the CAGA12-luciferase reporter plasmid; Prof. P. Cresswell (Department of Immunobiology, Yale University, New Haven, CT, USA) for a plasmid containing the viperin ORF; Dr. A. Groot (Maastro, Maastricht University, Maastricht, the Netherlands) for the pGluc-CMV plasmid; and Dr. S. Köhler (Anatomy and Embryology, Maastricht University, Maastricht, the Netherlands) for assistance with paraffin sectioning.

This work was financially supported by a bi-lateral cooperation grant (DN 82-304) from the Netherlands Organisation for Scientific Research (NWO) and the Deutsche Forschungsgemeinschaft (DFG) and by a grant from the Dutch Arthritis Association (LLP14). Dr. Peffers is supported by a Wellcome Trust Clinical Intermediate Fellowship (grant 107471). The authors declare no competing financial interests.

\section{Conflict of interest}

The authors declare that they have no conflicts of interest with the contents of this article. 


\section{References}

1 Seo, J. Y., Yaneva, R. \& Cresswell, P. Viperin: a multifunctional, interferon-inducible protein that regulates virus replication. Cell Host Microbe 10, 534-539, doi:10.1016/j.chom.2011.11.004 (2011).

2 Mattijssen, S. et al. Viperin mRNA is a novel target for the human RNase MRP/RNase P endoribonuclease. Cell Mol Life Sci 68, 2469-2480, doi:10.1007/s00018-010-0568-3 (2011).

3 Ridanpaa, M. et al. Mutations in the RNA component of RNase MRP cause a pleiotropic human disease, cartilage-hair hypoplasia. Cell 104, 195-203 (2001).

4 Mattijssen, S., Welting, T. J. \& Pruijn, G. J. RNase MRP and disease. Wiley Interdiscip Rev RNA 1, 102-116, doi:10.1002/wrna.9 (2010).

5 Makitie, O. \& Kostjukovits, S. in GeneReviews(R) (eds R. A. Pagon et al.) (1993).

6 Baron, J. et al. Short and tall stature: a new paradigm emerges. Nat Rev Endocrinol 11, 735-746, doi:10.1038/nrendo.2015.165 (2015).

7 Gill, T., Cai, T., Aulds, J., Wierzbicki, S. \& Schmitt, M. E. RNase MRP cleaves the CLB2 mRNA to promote cell cycle progression: novel method of mRNA degradation. Mol Cell Biol 24, 945-953 (2004).

8 Chang, D. D. \& Clayton, D. A. A mammalian mitochondrial RNA processing activity contains nucleus-encoded RNA. Science 235, 1178-1184 (1987).

9 Goldfarb, K. C. \& Cech, T. R. Targeted CRISPR disruption reveals a role for RNase MRP RNA in human preribosomal RNA processing. Genes Dev 31, 59-71, doi:10.1101/gad.286963.116 (2017).

10 Hermanns, P. et al. Consequences of mutations in the non-coding RMRP RNA in cartilage-hair hypoplasia. Hum Mol Genet 14, 3723-3740, doi:10.1093/hmg/ddi403 (2005).

11 Steinbusch, M. M. F. et al. Expression of RMRP RNA is regulated in chondrocyte hypertrophy and determines chondrogenic differentiation. Sci Rep 7, 6440, doi:10.1038/s41598-017-06809-5 (2017).

12 Atsumi, T., Miwa, Y., Kimata, K. \& Ikawa, Y. A chondrogenic cell line derived from a differentiating culture of AT805 teratocarcinoma cells. Cell Differ Dev 30, 109-116 (1990).

13 Caron, M. M. et al. Activation of NF-kappaB/p65 facilitates early chondrogenic differentiation during endochondral ossification. PLoS One 7, e33467, doi:10.1371/journal.pone.0033467 (2012).

14 Groot, A. J. et al. Regulated proteolysis of $\mathrm{NOTCH} 2$ and NOTCH3 receptors by ADAM10 and presenilins. Mol Cell Biol 34, 2822-2832, doi:10.1128/MCB.00206-14 (2014).

15 Dennler, S. et al. Direct binding of Smad3 and Smad4 to critical TGF beta-inducible elements in the promoter of human plasminogen activator inhibitor-type 1 gene. EMBO J 17, 3091-3100, doi:10.1093/emboj/17.11.3091 (1998). 
Pais, H. et al. Analyzing mRNA expression identifies Smad3 as a microRNA-140 target regulated only at protein level. RNA 16, 489-494, doi:10.1261/rna.1701210 (2010). Peffers, M. J., Beynon, R. J. \& Clegg, P. D. Absolute quantification of selected proteins in the human osteoarthritic secretome. Int J Mol Sci 14, 20658-20681, doi:10.3390/ijms141020658 (2013).

18 Thorpe, C. T. et al. Anatomical heterogeneity of tendon: Fascicular and interfascicular tendon compartments have distinct proteomic composition. Sci Rep 6, 20455, doi:10.1038/srep20455 (2016).

19 Peffers, M. J. et al. Proteomic analysis reveals age-related changes in tendon matrix composition, with age- and injury-specific matrix fragmentation. J Biol Chem 289, 25867-25878, doi:10.1074/jbc.M114.566554 (2014).

French, M. M., Rose, S., Canseco, J. \& Athanasiou, K. A. Chondrogenic differentiation of adult dermal fibroblasts. Ann Biomed Eng 32, 50-56 (2004).

Vizcaino, J. A. et al. The PRoteomics IDEntifications (PRIDE) database and associated tools: status in 2013. Nucleic Acids Res 41, D1063-1069, doi:10.1093/nar/gks1262 (2013).

22 Yao, Y. \& Wang, Y. ATDC5: an excellent in vitro model cell line for skeletal development. J Cell Biochem 114, 1223-1229, doi:10.1002/jcb.24467 (2013).

23 Hinson, E. R. \& Cresswell, P. The N-terminal amphipathic alpha-helix of viperin mediates localization to the cytosolic face of the endoplasmic reticulum and inhibits protein secretion. J Biol Chem 284, 4705-4712, doi:10.1074/jbc.M807261200 (2009).

24 Kronenberg, H. M. Developmental regulation of the growth plate. Nature 423, 332336, doi:10.1038/nature01657 (2003).

25 Dhillon, N. K., Peng, F., Ransohoff, R. M. \& Buch, S. PDGF synergistically enhances IFN-gamma-induced expression of CXCL10 in blood-derived macrophages: implications for HIV dementia. J Immunol 179, 2722-2730 (2007).

26 Nakao, A. et al. Identification of Smad7, a TGFbeta-inducible antagonist of TGF-beta signalling. Nature 389, 631-635, doi:10.1038/39369 (1997).

27 Kong, L. \& Liu, C. J. Mediation of chondrogenic and osteogenic differentiation by an interferon-inducible p202 protein. Cell Mol Life Sci 65, 3494-3506, doi:10.1007/s00018-008-8342-5 (2008).

28 Zhang, Y., Kong, L., Carlson, C. S. \& Liu, C. J. Cbfa1-dependent expression of an interferon-inducible p204 protein is required for chondrocyte differentiation. Cell Death Differ 15, 1760-1771, doi:10.1038/cdd.2008.112 (2008).

29 Morimoto, H., Baba, R., Haneji, T. \& Doi, Y. Double-stranded RNA-dependent protein kinase regulates insulin-stimulated chondrogenesis in mouse clonal chondrogenic cells, ATDC-5. Cell Tissue Res 351, 41-47, doi:10.1007/s00441-012-1521-6 (2013).

30 van Beuningen, H. M. et al. Inhibition of TAK1 and/or JAK can rescue impaired chondrogenic differentiation of human mesenchymal stem cells in osteoarthritis-like conditions. Tissue Eng Part A 20, 2243-2252, doi:10.1089/ten.TEA.2013.0553 (2014). 
Osaki, M. et al. The TATA-containing core promoter of the type II collagen gene (COL2A1) is the target of interferon-gamma-mediated inhibition in human chondrocytes: requirement for Stat1 alpha, Jak1 and Jak2. Biochem J 369, 103-115, doi:10.1042/BJ20020928 (2003).

Goldring, M. B., Fukuo, K., Birkhead, J. R., Dudek, E. \& Sandell, L. J. Transcriptional suppression by interleukin-1 and interferon-gamma of type II collagen gene expression in human chondrocytes. J Cell Biochem 54, 85-99, doi:10.1002/jcb.240540110 (1994).

Hinson, E. R. \& Cresswell, P. The antiviral protein, viperin, localizes to lipid droplets via its N-terminal amphipathic alpha-helix. Proc Natl Acad Sci U S A 106, 2045220457, doi:10.1073/pnas.0911679106 (2009).

Wang, X., Hinson, E. R. \& Cresswell, P. The interferon-inducible protein viperin inhibits influenza virus release by perturbing lipid rafts. Cell Host Microbe 2, 96-105, doi:10.1016/j.chom.2007.06.009 (2007).

35 Saitoh, T. et al. Antiviral protein Viperin promotes Toll-like receptor 7- and Toll-like receptor 9-mediated type I interferon production in plasmacytoid dendritic cells. Immunity 34, 352-363, doi:10.1016/j.immuni.2011.03.010 (2011).

Satish, L. et al. Expression analysis of human adipose-derived stem cells during in vitro differentiation to an adipocyte lineage. BMC Med Genomics 8, 41, doi:10.1186/s12920-015-0119-8 (2015).

37 Li, Z. et al. miR-200 family promotes podocyte differentiation through repression of RSAD2. Sci Rep 6, 27105, doi:10.1038/srep27105 (2016).

Amanatullah, D. F., Yamane, S. \& Reddi, A. H. Distinct patterns of gene expression in the superficial, middle and deep zones of bovine articular cartilage. J Tissue Eng Regen Med 8, 505-514, doi:10.1002/term.1543 (2014).

39 Grewal, T. S., Genever, P. G., Brabbs, A. C., Birch, M. \& Skerry, T. M. Best5: a novel interferon-inducible gene expressed during bone formation. FASEB J 14, 523-531 (2000).

40 Osawa, A. et al. Activation of genes for growth factor and cytokine pathways late in chondrogenic differentiation of ATDC5 cells. Genomics 88, 52-64, doi:10.1016/j.ygeno.2006.02.013 (2006).

41 Cristino, S. et al. Expression of CXC chemokines and their receptors is modulated during chondrogenic differentiation of human mesenchymal stem cells grown in three-dimensional scaffold: evidence in native cartilage. Tissue Eng Part A 14, $97-$ 105, doi:10.1089/ten.a.2007.0121 (2008).

42 Shahabuddin, S. et al. CXCR3 chemokine receptor-induced chemotaxis in human airway epithelial cells: role of p38 MAPK and PI3K signaling pathways. Am J Physiol Cell Physiol 291, C34-39, doi:10.1152/ajpcell.00441.2005 (2006).

$43 \mathrm{Yu}, \mathrm{J}$. S. et al. PI3K/mTORC2 regulates TGF-beta/Activin signalling by modulating Smad2/3 activity via linker phosphorylation. Nat Commun 6, 7212, doi:10.1038/ncomms8212 (2015). 
Chapter 3

44 Ulloa, L., Doody, J. \& Massague, J. Inhibition of transforming growth factorbeta/SMAD signalling by the interferon-gamma/STAT pathway. Nature 397, 710-713, doi:10.1038/17826 (1999).

45 Qiu, L. Q., Cresswell, P. \& Chin, K. C. Viperin is required for optimal Th2 responses and T-cell receptor-mediated activation of NF-kappaB and AP-1. Blood 113, 35203529, doi:10.1182/blood-2008-07-171942 (2009).

46 Groom, J. R. \& Luster, A. D. CXCR3 in T cell function. Exp Cell Res 317, 620-631, doi:10.1016/j.yexcr.2010.12.017 (2011).

47 Makitie, O., Kaitila, I. \& Savilahti, E. Susceptibility to infections and in vitro immune functions in cartilage-hair hypoplasia. Eur J Pediatr 157, 816-820 (1998). 
The anti-viral protein viperin regulates chondrogenic differentiation via CXCL10 protein secretion 
Laboratory for Experimental Orthopedics, Department of Orthopedic

Surgery, Maastricht University Medical Centre, Maastricht, the Netherlands 


\section{Chapter 4}

\section{Adaptation of the protein translational apparatus during ATDC5 chondrogenic differentiation}

Mandy M.F. Steinbusch, Guus G.H. van den Akker, Andy Cremers, Marjolein M.J. Caron, Lodewijk W. van Rhijn, Tim J.M. Welting 
Chapter 4

\section{Summary}

Ribosomes are universally responsible for translating mRNAs into protein. Ribosome synthesis is integrated with many cellular processes including proliferation, differentiation and oncogenic events. Chondrogenic progenitor cell proliferation and differentiation require a high cellular translational capacity to facilitate cartilaginous extracellular matrix production. However, how ribosome biogenesis is integrated in chondrogenic differentiation remains to be determined. In this study we investigated whether ribosome biogenesis is altered during the course of chondrogenic differentiation. 


\section{Introduction}

Ribosome biogenesis is a central cellular process required for production of ribosome subunits to translate proteins from mRNAs. From a number of genetic diseases it has become apparent that mutations in genes encoding key components of the ribosome biogenesis machinery (e.g. RMRP and cartilage hair hypoplasia ${ }^{1}$, Dyskerin and Dyskeratosis Congenita ${ }^{2}$, TCOF1 and Treacher-Collins syndrome ${ }^{3}$ ) or components of the mature ribosome (RPS14 and 5q syndrome 4 , RPS19 and Diamond Blackfan Anemia ${ }^{5}$, SBDS and SchwachmannDiamond syndrome ${ }^{6}$ ), interfere with cell function and are causative for developmental disorders and associated malignant conditions. The molecular dissection of these so-called ribosomopathies has unveiled many unprecedented molecular mechanisms and cell type specific effects of ribosome biogenesis and functions that dictate the capacity to synthesize proteins and support developmental processes and tissue homeostasis.

The majority of ribosomopathies is associated with disturbances of the development of the skeleton, leading to malformations and dwarfisms ${ }^{7}$. Skeletal development depends on endochondral ossification in the growth plates of the developing skeleton. This complex spatiotemporal cellular process encompasses the chondrocytic commitment of progenitor cells in the resting zone of the growth plate, followed by a proliferative burst of chondrocytes in the proliferative zone of the growth plate. Proliferative chondrocytes then terminally differentiate into hypertrophic chondrocytes. The extracellular matrix left behind by terminally differentiated hypertrophic chondrocytes is required for mineralization and ossification, enabling skeletal development ${ }^{8}$.

To accommodate the proliferative burst in the proliferative zone and the production of the cartilaginous extracellular matrix, a great amount of de novo synthesized proteins is needed, while the terminal hypertrophic differentiation calls for a major cellular proteomic change. We therefore expect that the cartilaginous phase of the endochondral ossification process is demanding for the growth plate chondrocyte's protein translation apparatus. Although a number of genes involved in ribosomopathies and their disease-causing mutations have been identified, the role of the ribosome biogenesis process and protein translation in general in the field of skeletal development remains to be elucidated.

It has been shown that small nucleolar RNA (snoRNAs), which guide post-transcriptional modification of ribosomal RNA (rRNA), are dynamically regulated upon murine embryonic 
stem cell differentiation ${ }^{9}$. In yeast and human cells, these rRNA modifications are required for ribosome translational fidelity ${ }^{10}$. As such, many aspects of small non-coding RNA function in development and disease remains to be elucidated ${ }^{11,12}$. Increasing amounts of evidence support the ribosome heterogeneity hypothesis ${ }^{13}$. One strong example is the Hox expression regulation by the large ribosomal protein subunit 38 in vertebrate tissue patterning ${ }^{14}$. We have previously shown that the ribosome biogenesis factor RMRP RNA is dynamically regulated during in vitro chondrogenic differentiation ${ }^{15}$. In addition, we demonstrated differential expression of snoRNAs in murine, equine and human cartilage disease (osteoarthritis) ${ }^{16}$. Further elucidation of ribosome biogenesis dynamics in developmental models, will lead to better understanding of ribosome function changes during cell commitment and their role in human disease.

To identify the potential involvement of ribosome biogenesis during chondrogenic differentiation, we investigated the expression dynamics of factors involved in ribosome biogenesis during chondrogenic differentiation and determined whether protein translation capacity differs between phases of chondrogenic differentiation in vitro.

Our data demonstrate differential expression of factors involved in rDNA transcription and post-transcriptional modification of rRNAs, as well dynamics of rRNA expression levels and protein translation, as a function of the course of ATDC5 chondrogenic differentiation. These data will aid in elucidating the dynamics of tuning the translational properties in cell differentiation.

\section{Materials and methods}

\section{Chondrogenic differentiation of ATDC5 cells}

ATDC5 cells ${ }^{17}$ (RIKEN BRC, Japan) were cultured in a humidified atmosphere at $37^{\circ} \mathrm{C}, 5 \% \mathrm{CO}_{2}$ in proliferation medium (DMEM/F12 (Invitrogen, Carlsbad, CA, USA), 5\% FCS (Sigma-Aldrich, St. Louis, MO, USA) and 1\% antibiotic/antimycotic (Invitrogen). Cells were plated at 6,400 cells $/ \mathrm{cm}^{2}$. After 24 hours chondrogenic differentiation was initiated by changing the medium to differentiation medium (proliferation medium supplemented with $10 \mu \mathrm{g} / \mathrm{ml}$ insulin (Sigma-Aldrich), $10 \mu \mathrm{g} / \mathrm{ml}$ transferrin (Roche, Basel, Switzerland) and $30 \mathrm{nM}$ sodium selenite 
(Sigma-Aldrich)). Differentiation medium was refreshed every two days for the first 10 days, and each day after day 10 , until cells were harvested at indicated time points.

\section{Real-time quantative PCR (RT-qPCR)}

Cells were harvested for RNA isolation with TRIzol reagent (Invitrogen). RNA was isolated from the TRIzol samples by collecting the aqueous phase after phase separation. RNA was precipitated with isopropanol $\left(30\right.$ minutes, $-80^{\circ} \mathrm{C}$ ) and centrifuged for 30 minutes at $20,000 \mathrm{x}$ g, $4^{\circ} \mathrm{C}$. RNA pellets were washed with $80 \%$ ethanol and potential DNA contamination was removed by DNase I (Roche) treatment $\left(1\right.$ hour, $\left.37^{\circ} \mathrm{C}\right)$. After subsequent ethanol precipitation, RNA was dissolved in $15 \mu \mathrm{L}$ DNase/RNase free water (Eurogentec, Seraing, Belgium). RNA quantity and purity were determined spectrophotometrically (Biodrop, Isogen Life Sciences, Utrecht, the Netherlands). DNA-free total RNA was reverse transcribed using standard procedures and random hexamer priming as described previously ${ }^{18}$. RT-qPCR was performed in 96-well optical plates. For each cDNA sample a mix was prepared consisting of Mesagreen qPCR Mastermix Plus for SYBR Green (Eurogentec) and $300 \mathrm{nM}$ forward and reverse oligonucleotides. Serially diluted standard curves were utilized to quantify gene expression in the samples. A Biorad CFX96 Real-Time PCR Detection System was used for amplification using the following protocol: denaturation at $95^{\circ} \mathrm{C}$ for 5 minutes, followed by 50 cycles of amplification $\left(15\right.$ seconds $95^{\circ} \mathrm{C}$ and 45 seconds $60^{\circ} \mathrm{C}$ ) followed by a dissociation curve. Data were analyzed using Biorad CFX Manager Software version 3.1, based on the relative quantification of the expression of the target gene normalized to $\beta$ Actin housekeeping gene. Primer sequences are provided in Table 1.

Table 1. RT-qPCR primer sequences

\begin{tabular}{|l|l|l|}
\hline Gene Symbol & Forward & Reverse \\
\hline Actb & CCGAGCGCGAGATCGT & TGGCCATCTCGTTCTCGAA \\
\hline Col2a1 & TGGGTGTTCTATTTATTTATTGTCTTCCT & GCGTTGGACTCACACCAGTTAGT \\
\hline Col10a1 & CATGCCTGATGGCTTCATAAA & AAGCAGACACGGGCATACCT \\
\hline Dkc1 & CCACTCGTTTGGTGAAATCACA & AGCCGGACAATTCCAACATACT \\
\hline
\end{tabular}




\begin{tabular}{|l|l|l|}
\hline Gene Symbol & Forward & Reverse \\
\hline Fbl & TGGAGCCGCATCGTCAT & CAAGGGCATCCTCCTTTCC \\
\hline Runx 2 & GACGAGGCAAGAGTTTCACC & GGACCGTCCACTGTCACTTT \\
\hline 185 rRNA & AGTCCCTGCCCTTTGTACACA & CGCTCAGACAGGCGTAGCCC \\
\hline 285 rRNA & GCCATGGTAATCCTGCTCAGTAC & GCTCCTCAGCCAAGCACATAC \\
\hline Sox9 & AGTACCCGCACCTGCACAAC & TACTTGTAGTCCGGGTGGTCTTTC \\
\hline Ubf-1 & CAGGACCGTGCAGCATATAAAG & GCCTCGCAGCTTGGTCAT \\
\hline
\end{tabular}

\section{Immunoblotting}

Cells were washed three times with $0.9 \% \mathrm{NaCl}$. The cells were lysed in RIPA buffer (150 mM $\mathrm{NaCl}, 1 \% \mathrm{NP}-40,0.5 \%$ sodium deoxycholate, $0.1 \% \mathrm{SDS}, 50 \mathrm{mM}$ Tris $\mathrm{pH}$ 8.0, 5.0 mM EDTA pH 8.0, $0.5 \mathrm{mM}$ dithiothreitol (DTT) and $1 \mathrm{mM}$ phenylmethylsulfonylfluoride (PMSF)). Samples were homogenized by sonication (Soniprep $150 \mathrm{MSE}$ ) on ice using the following protocol: 14 cycles of 1 second sonication followed by a 1 second interval, amplitude 10. Cell debris was removed by means of centrifugation at 15,000 RPM for 10 minutes on $4^{\circ} \mathrm{C}$. Total protein concentration was determined with a BCA assay (Sigma-Aldrich). Samples were separated by gel electrophoresis and transferred to nitrocellulose membranes by electro blotting. The following primary antibodies were used for immunodetection:

Mouse monoclonal anti-Dyskerin (C-11, Santa Cruz, Dallas, TX, USA, \#SC-365731; 1:200 dilution), rabbit monoclonal anti-Fibrillarin (EPR10822(B), Abcam, Cambridge, UK, \#AB154806; 1:1000 dilution), mouse monoclonal anti-UBF1 (F-9, Santa Cruz \#SC-13125; 1:250 dilution), rabbit polyclonal anti-Sox9 (Abcam \#AB3697; 1:100 dilution) and mouse monoclonal anti-Puromycin (12D10, Merck Millipore, Billerica, MA, USA, \#MABE343; 1:1000 dilution). As a control mouse monoclonal anti-Histone H3 (Abcam \#24834; 1:1000 dilution) or mouse monoclonal anti- $\alpha$-Tubulin (B-5-1-2, Sigma-Aldrich \#T6074; 1:10,000 dilution) were used. HRP-conjugated polyclonal rabbit anti-mouse or swine anti-rabbit (Dako) were applied 
as a secondary antibody and the bound antibodies were detected by enhanced chemiluminescence (ECL) (Bio-Rad Chemidoc XRS+).

\section{SYBR Green assay for DNA content quantification}

Ribosomal RNA content per cell was quantified by calculating 18S, $5.8 \mathrm{~S}$ and $28 \mathrm{~S}$ rRNA RTqPCR signals in six biological replicates (equal volumes of aqueous phase RNA were isolated and equal volumes of RNA were reverse transcribed to CDNA) relative to the DNA content in six biological replicates. DNA concentrations in equal volumes of papaine digestion buffer (100 mM phosphate buffer $\left(\mathrm{NaH}_{2} \mathrm{PO}_{4}\right.$ (VWR, Amsterdam, the Netherlands) and $\mathrm{Na}_{2} \mathrm{HPO}_{4}$ * $2 \mathrm{H}_{2} \mathrm{O}$ (VWR), pH 6.5), 5 mM L-cysteine*HCl (Sigma-Aldrich), 5 mM EDTA (VWR), $33.33 \mu \mathrm{g} / \mu \mathrm{l}$ papaine (Sigma-Aldrich)) were determined in samples from day 0, 7 and 14 of ATDC5 chondrogenic differentiation using the SYBR Green assay (Invitrogen). Prior to measurement, samples were diluted in TE buffer (10 mM Tris/HCl pH 8 and $1 \mathrm{mM}$ EDTA; day 0; 1:100 dilution, day 7 and day $14 ; 1: 1000$ dilution). A serially diluted standard curve of calf thymus genomic control DNA (Invitrogen) in TE buffer was included to quantify the DNA concentration in the samples. Standards were prepared to contain the same amount of papaine digestion buffer as the samples. SYBR Green was diluted 10,000 times in TE buffer and $100 \mu \mathrm{l}$ was added to $100 \mu \mathrm{l}$ of the above prepared samples and standards. After 10 minutes incubation fluorescence was determined using a Spectramax M2E (Molecular Devices, Sunnyvale, CA, USA) microplate reader with an excitation of $488 \mathrm{~nm}$ and an emission of $522 \mathrm{~nm}$ and DNA concentration was calculated using the standard curve.

\section{Determination of active translation by puromycin incorporation}

Prior to harvesting at day $0,3,7,10$ and 14, ATDC5 cells were incubated with $10 \mu \mathrm{g} / \mathrm{ml}$ puromycin (Sigma-Aldrich) in normal proliferation (day 0) or differentiation medium (day 3, 7,10 and 14 ) for 15 minutes at $37^{\circ} \mathrm{C}, 5 \% \mathrm{CO}_{2}$ in a humidified atmosphere. After puromycin was incorporated for exactly 15 minutes, cells were washed twice with $0.9 \% \mathrm{NaCl}$ and harvested for Western blotting with RIPA lysis buffer (see section Immunoblotting). The western blot whole lane puromycin signal (volume intensity; vol. INT) was quantified for 


\section{Chapter 4}

each sample and corrected for the quantified anti- $\alpha$-TUBULIN signal (housekeeper) using Bio-Rad Image Lab Software 5.2.1.

\section{snoRNA sequencing}

SnoRNA expression during ATDC5 differentiation was analyzed by small RNA Next Generation Sequencing (NGS) of samples acquired from day 0 (progenitor stage), day 7 (chondrogenic stage) and day 14 (hypertrophic stage). Total RNA was isolated and after library preparation (NEBNext library generation kit, New England Biolabs (NEB), Ipswich, MA, USA) inserts $<200 \mathrm{nt}$ were selected using an automated gel cutter. One $\mu \mathrm{g}$ of total RNA was converted into NGS libraries using the NEBNext library generation kit (NEB), using a protocol (Exiqon, Vedbæk, Denmark) to increase the 5' and 3' ligation of modified small RNA ends. Each individual RNA sample had adapters ligated to its $3^{\prime}$ and $5^{\prime}$ ends and was converted to cDNA. Then, the cDNA was pre-amplified with specific primers containing sample specific indexes (Exiqon). After a 15 cycle pre-PCR the libraries were purified on QiaQuick column (Qiagen, Hilden, Germany) and the insert efficiency was evaluated on a Bioanalyzer 2100 instrument (Agilent, Santa Clara, CA, USA) on a high sensitivity DNA chip (Agilent). The cDNA libraries were size fractioned on a LabChip XT (Caliper Life Sciences, Waltham, MA, USA) and 15-200 bp inserts were excised according to the manufacturer's protocol (Caliper). Samples were then quantified using $\mathrm{qPCR}$ and a concentration standard. Based on the quality of the inserts and the concentration measurements the libraries were pooled in equimolar concentrations. The library pool was quantified with qPCR and an optimal concentration of the library pool (Illumina) was used to generate the clusters on the surface of a flow cell before sequencing (using v3 sequencing methodology according to the manufacturer's instructions). Libraries were then sequenced on the Illumina platform. After filtering and normalization, using the trimmed mean of the $M$-values method, based on log-fold and absolute gene wise changes in expression levels between samples ${ }^{19}$, reads that mapped to snoRNAs were analyzed for differential expression. 


\section{Polysome fractionation}

Polyosome fractionation was carried out essentially as described previously ${ }^{20}$. Three $15 \mathrm{~cm}$ plates with ATDC5 cells were used to generate a single sample at day 0 , two plates at day 7 and one plate at day 14. At the day of sample collection, cells were pre-treated for 5 minutes with $100 \mu \mathrm{g} / \mathrm{ml}$ Cycloheximide (Sigma), washed twice in $0.9 \% \mathrm{NaCl}$ with cycloheximide and collected by scraping with a rubber policeman in cold $0.9 \% \mathrm{NaCl}$. Pelleted cells were lysed for 10 minutes in $1.8 \mathrm{ml}$ polysome extraction buffer $(20 \mathrm{mM}$ Tris- $\mathrm{HCl}(\mathrm{pH} 7.5), 100 \mathrm{mM} \mathrm{KCl}, 5$ $\mathrm{mM} \mathrm{MgCl} 2,0.5 \%$ Nonidet P-40, $100 \mu \mathrm{g} / \mathrm{ml}$ Cycloheximide, complete protease inhibitor cocktail (Roche) and RNasin (Promega, 40U/ml)) on ice. Nuclei and cellular debris were removed by centrifugation at $12.000 \mathrm{x}$ for 10 minutes at $4{ }^{\circ} \mathrm{C}$ and $9 / 10^{\text {th }}$ of the total volume was transferred to fresh tubes and measured spectrophotometrically. Ten percent input was set aside and stored at $-80^{\circ} \mathrm{C}$. Linear $10-50 \%$ sucrose gradients were made using the Gradient Master (BioComp) in SW41 ultracentrifuge tubes (Seton). A fixed amount of $160 \mu \mathrm{g}$ cytoplasmic extract was loaded to each gradient, for each sample in the same volume. Gradients were run on a Beckman L60 ultra-centrifuge at $39.000 \mathrm{rpm}$ for 1.5 hours at $4^{\circ} \mathrm{C}$ with max acceleration and deceleration 9. Samples were fractionated into $24 \times 0.5 \mathrm{ml}$ fractions using a Piston Gradient fractionator (BioComp) and fraction collector (Gilson FC203B) with continuous A260 monitoring (Triax FC-1).

\section{Results}

\section{Differential expression of snoRNAs during ATDC5 chondrogenic differentiation}

Previously we have shown that the snoRNA RMRP is differentially expressed during chondrogenic differentiation ${ }^{15}$ and interference with its function led to important changes in the outcome of chondrogenic differentiation with consequences for ribosomal RNA levels and human disease. To identify additional snoRNAs that may play a role in chondrogenic differentiation, we now performed small RNA sequencing ( $<200 \mathrm{nt})$ in ATDC5 chondrogenic differentiation at day 0,7 and 14. Expression of at least 228 different snoRNA species was 
detected (Figure 1). We found 21 different snoRNAs differentially expressed between day 0 and day 7 in differentiation (Figure 1; $\Delta t 0-t 7$ ) of which 14 were box C/D snoRNAs and 7 were box H/ACA snoRNAs. Differential expression of 23 snoRNAs was detected between day 7 and 14 (Figure 1; $\Delta \mathrm{t} 7-\mathrm{t} 14$ ) of which 16 were box C/D snoRNAs and 7 box H/ACA snoRNAs. Finally, differential expression of 43 snoRNAs was found when comparing day 0 with day 14 data (Figure 1; $\Delta \mathrm{t} 0$-t14), with 33 of which were box C/D snoRNAs and 10 were box H/ACA snoRNAs. A full overview of the differentially expressed snoRNAs and their putative ribosomal RNA ( $r R N A$ ) targets (2'O-ribose methylation or pseudouridylation of specific rRNA nucleotides) is presented in Table $\mathbf{2}$.
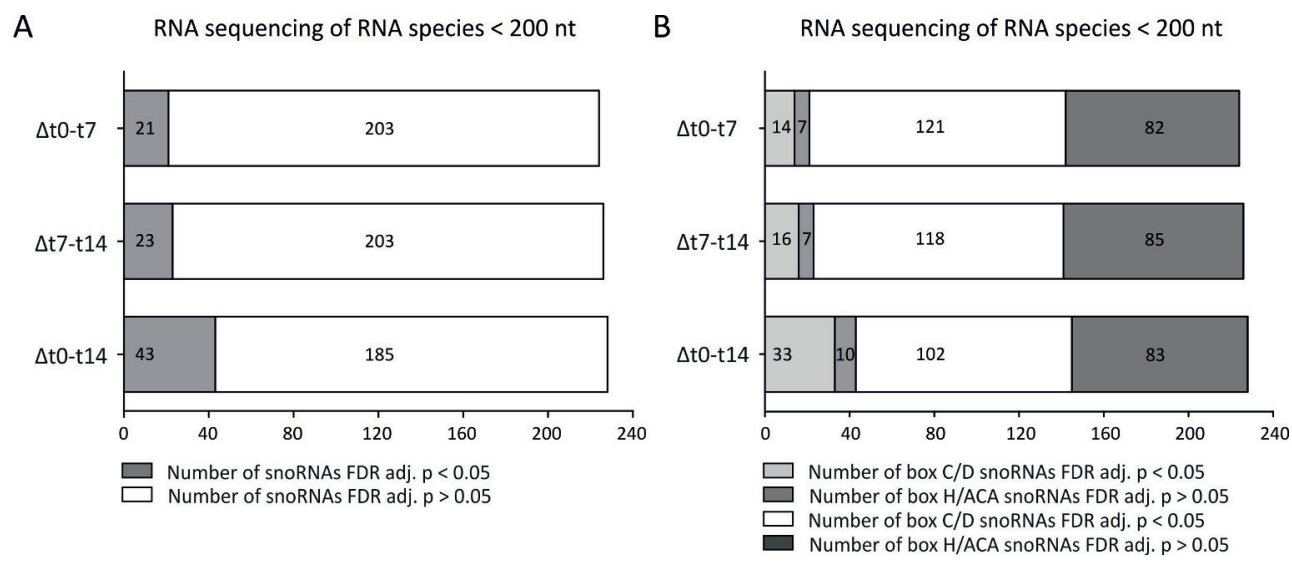

Figure 1. Differential expression of snoRNAs during different phases of chondrogenic differentiation. ATDC5 cells were differentiated in the chondrogenic lineage for 0,7 or 14 days and RNA-Seq of RNA species $<200$ nt was performed. A) The number of snoRNAs and differentially expressed (FDR adjusted $p$-value $<0.05, \log F C>1$ or $<-1$ ) snoRNAs identified with RNA-Seq between day 0 and day 7 (initiation of chondrogenic differentiation), day 7 and day 14 (hypertrophic differentiation) and day 0 and day 14 (progenitor versus hypertrophic chondrocyte). B) Subdivision between box C/D versus box H/ACA snoRNAs in differentially and non-differentially expressed snoRNAs as identified by RNA-sequencing. 


\section{Table 2. Differentially expressed snoRNAs during different phases of ATDC5}

chondrogenic differentiation. The ATDC5 differentiation phase dependent significantly differentially expressed snoRNAs are indicated per contrast ( $\Delta \mathrm{t} 0-\mathrm{t} 7, \Delta \mathrm{t} 7 \mathrm{-t} 14, \Delta \mathrm{t} 0-\mathrm{t} 14)$. For each snoRNA the RNA target is indicated, as well as the counts, fold-differences and significance. RNA target information was acquired from snoRNABase ${ }^{21}$.

\begin{tabular}{|c|c|c|c|c|c|c|}
\hline $\begin{array}{l}\triangle \text { day } 0-7 \\
\text { SnoRNA }\end{array}$ & Box & RNA target & $\begin{array}{l}\text { Count } \\
\text { day } 0\end{array}$ & $\begin{array}{l}\text { Count } \\
\text { day } 7\end{array}$ & LogFC & $\begin{array}{l}\text { FDR } \\
\text { adjusted } \\
\text { p-value }\end{array}$ \\
\hline SNORA24 & H/ACA & 18S rRNA U863 + U609 & 45 & 7 & -2.84 & 0.001 \\
\hline SNORD1C & $C / D$ & 28S rRNA G4362 & 29 & 7 & -2.27 & 0.001 \\
\hline SNORD101 & $C / D$ & Unknown / orphan & 1365 & 584 & -1.51 & 0.006 \\
\hline SNORD1A & $C / D$ & 28S rRNA G4362 & 14 & 3 & -2.20 & 0.007 \\
\hline SNORD1B & $C / D$ & 28S rRNA G4362 & 34 & 12 & -1.68 & 0.007 \\
\hline SNORD80 & $C / D$ & 28S rRNA A1521 + G1612 & 6 & 1 & -2.77 & 0.010 \\
\hline SNORA66 & H/ACA & 18S rRNA U119 & 32 & 13 & -1.53 & 0.013 \\
\hline SNORA12 & H/ACA & U6 snRNA U40 & 185 & 73 & -1.53 & 0.013 \\
\hline SNORD23 & $C / D$ & Unknown / orphan & 25 & 6 & -2.27 & 0.013 \\
\hline SNORA31 & H/ACA & $\begin{array}{l}\text { 18S rRNA U218 + 28S } \\
\text { rRNA U3713 }\end{array}$ & 40 & 128 & 1.51 & 0.014 \\
\hline SNORA2 & H/ACA & 28S rRNA U4263 + U4282 & 12 & 4 & -1.71 & 0.023 \\
\hline SNORD46 & $C / D$ & 28S rRNA A3739 & 16913 & 8541 & -1.45 & 0.024 \\
\hline SNORD100 & $C / D$ & 18S rRNA G436 & 47 & 23 & -1.30 & 0.026 \\
\hline SNORD61 & C/D & 18S rRNA U1442 & 1724 & 4729 & 1.18 & 0.031 \\
\hline SNORD1 & $C / D$ & 28S rRNA G4362 & 1870 & 875 & -1.70 & 0.034 \\
\hline SNORD36 & $C / D$ & $\begin{array}{l}\text { 18S rRNA A668 + 28S rRNA } \\
\text { A3703 }\end{array}$ & 1033 & 593 & -1.17 & 0.036 \\
\hline SNORA73 & H/ACA & Unknown / orphan & 54 & 24 & -1.53 & 0.039 \\
\hline SNORD53 & $C / D$ & 28S rRNA C3848 & 1952 & 1124 & -1.11 & 0.040 \\
\hline SNORD50B & $C / D$ & Unknown / orphan & 4440 & 14264 & 1.36 & 0.042 \\
\hline SNORD30 & $C / D$ & 28S rRNA A3804 & 22986 & 58998 & 1.17 & 0.045 \\
\hline SNORA46 & H/ACA & 18S rRNA U649 & 190 & 101 & -1.23 & 0.049 \\
\hline $\begin{array}{l}\Delta \text { day 7-14 } \\
\text { snoRNA }\end{array}$ & Box & RNA target & $\begin{array}{l}\text { Count } \\
\text { day } 7\end{array}$ & $\begin{array}{l}\text { Count } \\
\text { day } 14\end{array}$ & LogFC & $\begin{array}{l}\text { FDR } \\
\text { adjusted } \\
\text { p-value }\end{array}$ \\
\hline SNORD103 & C/D & 18S rRNA G601 & 355 & 73 & -2.14 & 0.001 \\
\hline SNORD30 & $C / D$ & 28S rRNA A3804 & 58998 & 13859 & -2.03 & 0.001 \\
\hline SNORD99 & $C / D$ & 28S rRNA A2774 & 155 & 34 & -2.00 & 0.001 \\
\hline SNORD36 & $C / D$ & $\begin{array}{l}\text { 18S rRNA A668 + 28S rRNA } \\
\text { A3703 }\end{array}$ & 593 & 144 & -1.98 & 0.001 \\
\hline SNORD55 & $C / D$ & 28S rRNA C2791 & 554 & 132 & -1.92 & 0.001 \\
\hline SNORD52 & $C / D$ & 28S rRNA U3904 & 20290 & 5365 & -1.81 & 0.002 \\
\hline SNORD66 & $C / D$ & 18S rRNA C1272 & 7071 & 1833 & -1.88 & 0.006 \\
\hline SNORD21 & $C / D$ & 28S rRNA G1303 & 14514 & 4580 & -1.56 & 0.006 \\
\hline SNORA28 & H/ACA & 18S rRNA U815 + U866 & 151 & 401 & 1.48 & 0.009 \\
\hline SNORA40 & $\mathrm{H} / \mathrm{ACA}$ & $\begin{array}{l}\text { 18S rRNA U1174 + 28S } \\
\text { rRNA U4546 }\end{array}$ & 14 & 43 & 1.72 & 0.010 \\
\hline
\end{tabular}




\begin{tabular}{|c|c|c|c|c|c|c|}
\hline SNORD88 & $C / D$ & 28S rRNA C3680 & 11 & 2 & -2.26 & 0.011 \\
\hline SNORD42B & $C / D$ & 18S rRNA U116 & 117 & 34 & -1.60 & 0.012 \\
\hline SNORD24 & $C / D$ & 28S rRNA C2338 + C2352 & 27793 & 7902 & -1.67 & 0.014 \\
\hline SNORA62 & $\mathrm{H} / \mathrm{ACA}$ & 28S rRNA U3830 + U3832 & 13 & 38 & 1.61 & 0.020 \\
\hline SNORD3 & $C / D$ & Unknown / orphan & 1218 & 220 & -2.20 & 0.020 \\
\hline SNORD59 & $C / D$ & 18S rRNA A1031 & 191 & 69 & -1.33 & 0.028 \\
\hline SCARNA3B & H/ACA & U6 snRNA U40 & 2 & 8 & 2.07 & 0.030 \\
\hline SNORA30 & $\mathrm{H} / \mathrm{ACA}$ & 28S rRNA U4643 & 18 & 49 & 1.43 & 0.033 \\
\hline SNORD85 & $C / D$ & 18S rRNA G601 & 44 & 16 & -1.32 & 0.038 \\
\hline SNORD18 & $C / D$ & 28S rRNA A1313 & 4634 & 1853 & -1.27 & 0.039 \\
\hline SNORA36 & $\mathrm{H} / \mathrm{ACA}$ & 18S rRNA U105 + U1244 & 44 & 99 & 1.26 & 0.044 \\
\hline SNORA26 & $\mathrm{H} / \mathrm{ACA}$ & 28S rRNA U4522 & 6 & 1 & -1.87 & 0.044 \\
\hline SNORD45 & $C / D$ & 18S rRNA A159 + U172 & 89488 & 39837 & -1.09 & 0.049 \\
\hline $\begin{array}{l}\Delta \text { day } 0-14 \\
\text { snoRNA }\end{array}$ & Box & RNA target & $\begin{array}{l}\text { Count } \\
\text { day } 0\end{array}$ & $\begin{array}{l}\text { Count } \\
\text { day } 14\end{array}$ & LogFC & $\begin{array}{l}\text { FDR } \\
\text { adjusted } \\
\text { p-value }\end{array}$ \\
\hline SNORD36 & $C / D$ & $\begin{array}{l}\text { 18S rRNA A668 + 28S rRNA } \\
\text { A3703 }\end{array}$ & 1033 & 144 & -3.18 & 4.79E-07 \\
\hline SNORA24 & $\mathrm{H} / \mathrm{ACA}$ & 18S rRNA U609 + U863 & 45 & 5 & -3.50 & $2.85 \mathrm{E}-06$ \\
\hline SNORD101 & $C / D$ & Unknown / orphan & 1365 & 312 & -2.36 & 3.39E-05 \\
\hline SNORD1C & $C / D$ & 28S rRNA G4362 & 29 & 3 & -3.25 & $5.93 \mathrm{E}-05$ \\
\hline SNORD55 & $C / D$ & 28S rRNA C2791 & 472 & 132 & -2.08 & $2.69 \mathrm{E}-04$ \\
\hline SNORA30 & $\mathrm{H} / \mathrm{ACA}$ & 28S rRNA U4643 & 7 & 49 & 2.45 & $2.99 \mathrm{E}-04$ \\
\hline SNORD1B & $C / D$ & 28S rRNA G4362 & 34 & 7 & -2.49 & 0.001 \\
\hline SNORD53 & $C / D$ & 28S rRNA C3848 & 1952 & 643 & -1.87 & 0.001 \\
\hline SNORD42A & $C / D$ & 18S rRNA U116 & 834 & 274 & -1.80 & 0.002 \\
\hline SNORD2 & $C / D$ & 28S rRNA G1509 & 22228 & 6971 & -1.86 & 0.002 \\
\hline SNORD42B & $C / D$ & 18S rRNA U116 & 134 & 34 & -2.09 & 0.002 \\
\hline SNORD81 & $C / D$ & 28S rRNA A391 & 14 & 2 & -2.54 & 0.002 \\
\hline SNORD82 & $C / D$ & 18S rRNA A1678 & 41432 & 15120 & -1.76 & 0.003 \\
\hline SNORD99 & $C / D$ & 28S rRNA A2774 & 105 & 34 & -1.80 & 0.003 \\
\hline SNORD100 & $C / D$ & 18S rRNA G436 & 47 & 15 & -1.83 & 0.003 \\
\hline SNORA47 & $\mathrm{H} / \mathrm{ACA}$ & 28S rRNA U1766 & 7 & 35 & 1.98 & 0.004 \\
\hline SNORD31 & $C / D$ & 28S rRNA A4166 & 758 & 291 & -1.58 & 0.005 \\
\hline SNORD3B2 & $C / D$ & Unknown / orphan & 1324 & 344 & -2.03 & 0.005 \\
\hline SNORD46 & $C / D$ & 28S rRNA A3739 & 16913 & 6417 & -1.80 & 0.005 \\
\hline SNORD96A & $C / D$ & 5.8S rRNA G75 & 293 & 109 & -1.61 & 0.005 \\
\hline SNORD102 & $C / D$ & 28S rRNA G4020 & 12662 & 4647 & -1.67 & 0.005 \\
\hline SNORD21 & $C / D$ & 28S rRNA G1303 & 10972 & 4580 & -1.61 & 0.006 \\
\hline SNORD1A & $C / D$ & 28S rRNA G4362 & 14 & 2 & -2.47 & 0.006 \\
\hline SNORD59 & $C / D$ & 18S rRNA A1031 & 191 & 69 & -1.63 & 0.007 \\
\hline SNORA57 & $\mathrm{H} / \mathrm{ACA}$ & U5 snRNA U43 & 22 & 76 & 1.59 & 0.009 \\
\hline SNORD1 & $C / D$ & 28S rRNA G4362 & 1870 & 629 & -2.11 & 0.014 \\
\hline SNORA54 & $\mathrm{H} / \mathrm{ACA}$ & 28S rRNA U3801 + U4539 & 10 & 38 & 1.75 & 0.016 \\
\hline SNORD10 & $C / D$ & $\begin{array}{l}\text { U6 snRNA C77 + 28S rRNA } \\
\text { C3787 }\end{array}$ & 1612 & 5003 & 1.36 & 0.017 \\
\hline SNORD79 & $C / D$ & 28S rRNA A3809 & 54 & 25 & -1.43 & 0.020 \\
\hline SNORA26 & $\mathrm{H} / \mathrm{ACA}$ & U2 snRNA U39 + U41 & 14 & 48 & 1.52 & 0.021 \\
\hline SNORD4A & $C / D$ & 18S rRNA U121 & 67 & 19 & -1.83 & 0.021 \\
\hline SNORD28 & $C / D$ & 18S rRNA C1391 & 6570 & 2962 & -1.35 & 0.021 \\
\hline
\end{tabular}




\begin{tabular}{|l|l|l|l|l|l|r|}
\hline SNORA28 & H/ACA & 18S rRNA U815 + U866 & 131 & 401 & 1.36 & 0.023 \\
\hline SNORD45 & C/D & 18S rRNA A159 + U172 & 76766 & 39837 & -1.20 & 0.028 \\
\hline SNORD33 & C/D & 18S rRNA U1326 & 4316 & 2211 & -1.22 & 0.029 \\
\hline ScaRNA14 & H/ACA & U2 snRNA U7 & 2 & 11 & 1.87 & 0.031 \\
\hline SNORD24 & C/D & 28S rRNA C2338 + C2352 & 8268 & 4279 & -1.20 & 0.032 \\
\hline SNORA36 & H/ACA & 18S rRNA U105 + U1244 & 34 & 99 & 1.32 & 0.035 \\
\hline SNORD52 & C/D & 28S rRNA U3904 & 10449 & 5365 & -1.17 & 0.041 \\
\hline SNORD86 & C/D & Unknown / orphan & 217 & 104 & -1.23 & 0.044 \\
\hline SNORD94 & C/D & U6 snRNA C62 & 220 & 569 & 1.15 & 0.046 \\
\hline SNORD80 & C/D & 28S rRNA A1521 + G1612 & 6 & 2 & -1.90 & 0.048 \\
\hline & H/ACA & $\begin{array}{l}\text { 18S rRNA U218 + 28S } \\
\text { rRNA U3713 }\end{array}$ & 40 & 109 & 1.30 & 0.049 \\
\hline
\end{tabular}

The majority of snoRNAs is involved in the post-transcriptional modification of rRNAs, and to map which rRNA domains are putative targets of the differentially expressed snoRNAs found in our RNAseq analysis, we plotted the rRNA targets of the differentially expressed snoRNAs in the 2D rRNA structure of $18 \mathrm{~S}$ (Figure 2A), 5.8S and 28S rRNAs (Figure 2B). Target sites of differentially expressed snoRNAs between day 0 and 7 are visualized in pink; $\Delta$ day $7-14$ in green and $\Delta$ day $0-14$ in blue. This revealed that differentially expressed snoRNAs, amongst others, target post-transcriptional modifications of the 18S rRNA decoding center (DC; Figure 2A, open black circles indicate the 5 helices of the $18 \mathrm{~S} \mathrm{DC)}$ and of the $28 \mathrm{~S}$ rRNA peptidyl transferase center (PTC; Figure 2B, open black circles indicate the helices of the 28S PTC). 


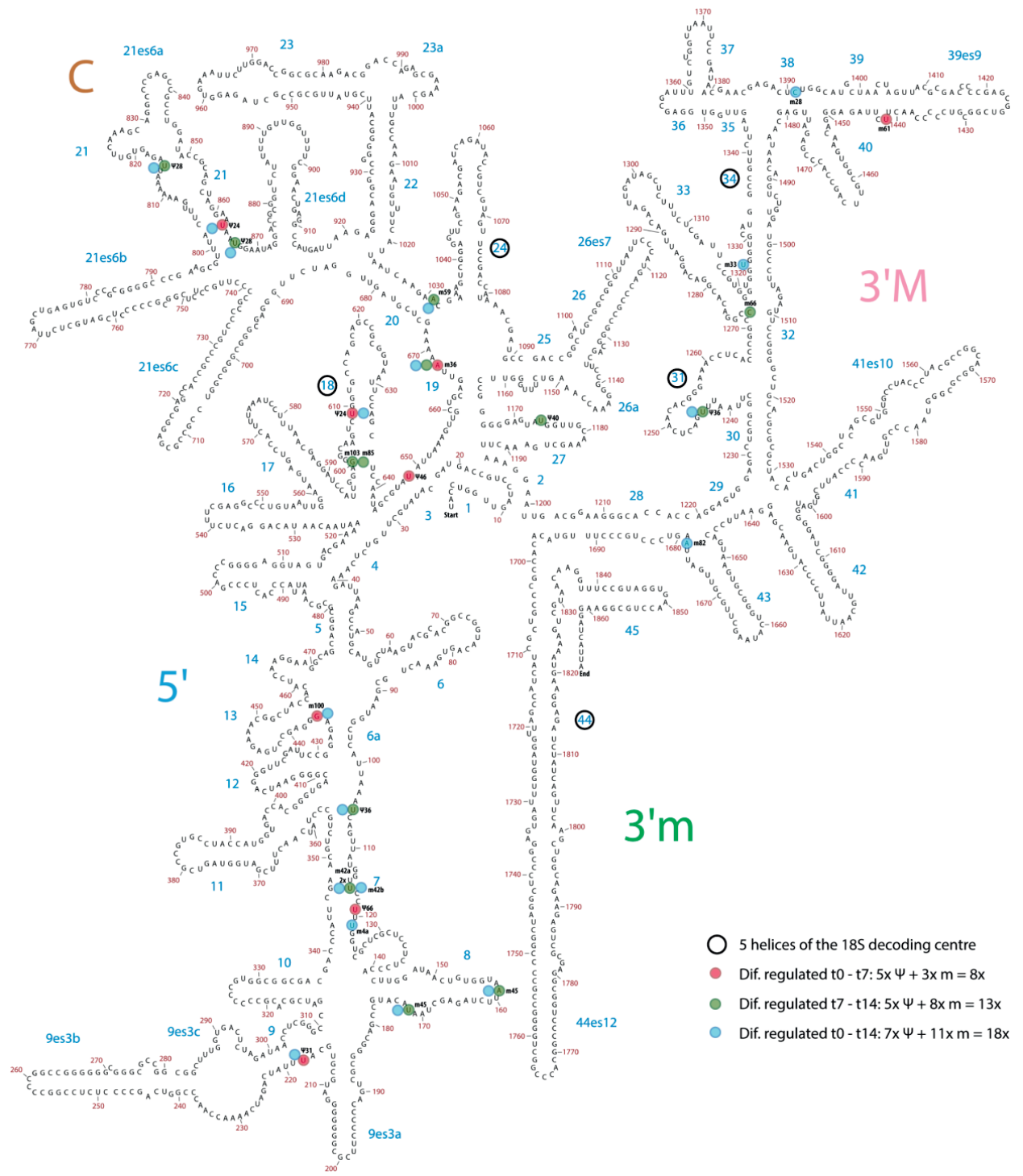

Figure 2A. Target sites of differentially expressed snoRNAs in the $18 \mathrm{~S}$ small ribosomal subunit. Target sites of differentially expressed snoRNAs between day 0 and 7 are visualized in pink; $\Delta$ day $7-14$ in green and $\Delta$ day $0-14$ in blue. Specific snoRNAs are indicated ( $m=2$ '-O ribose methylation, $\Psi=$ pseudouridylation). Open black circles indicate the 5 helices of the 18 S decoding centre. SNORA40 in helix 27 is indicated by the black box. 2-D rRNA structure was adapted from Apollo Chemistry Gatech Ribovision Ribosome Visualization Suite ${ }^{22}$. 


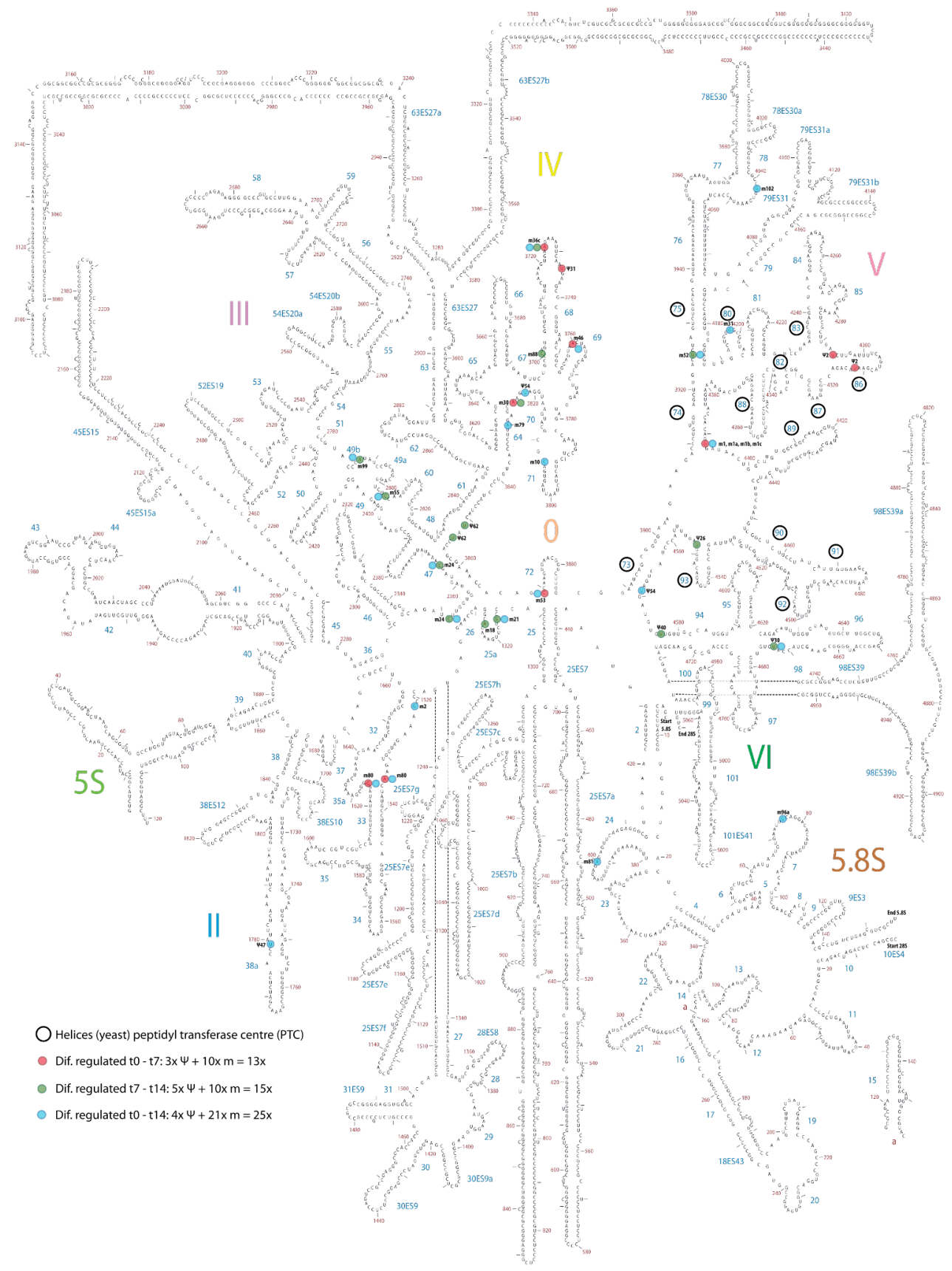

Figure 2B. Target sites of differentially expressed snoRNAs in the large ribosomal subunit (5S, $5.8 S$ and $28 S$ ). Target sites of differentially expressed snoRNAs between day 0 and 7 are visualized in pink; $\Delta$ day $7-14$ in green and $\Delta$ day $0-14$ in blue. Specific snoRNAs are indicated (m $=2^{\prime}-0$ ribose methylation, $\Psi=$ pseudouridylation). Open black circles indicate the helices of the $28 \mathrm{~S}$ 


\section{Chapter 4}

peptidyl transferase center. 2-D rRNA structure was adapted from Apollo Chemistry Gatech Ribovision Ribosome Visualization Suite ${ }^{22}$.

SnoRNAs site-directionally guide the modification of their rRNA targets by sequence complementarity. However, the 2'O-ribose methylase and pseudouridylase activity that ultimately leads to the rRNA post-transcriptional modifications is carried out by fibrillarin and dyskerin, respectively. With fibrillarin being a core component of Box C/D snoRNPs and dyskerin of Box H/ACA snoRNPs. Since we found differential expression of specific snoRNAs in different phases of ATDC5 chondrogenic differentiation, we asked whether the expression of their enzymatic core proteins also alters as a function of chondrogenic differentiation. ATDC5 were differentiated into the chondrogenic lineage and expression of chondrogenic markers was first measured to confirm chondrogenic commitment (Figure 3). The mRNA expression of Sox9, Col2a1, Runx2 and Col10a1 (Figure 3A-D) was induced over a course of 14 days, with chondrogenic differentiation represented by induction of Sox9 and Col2a1 expression and becoming apparent from day 5-6-7 onward. This was further confirmed by Sox9 immunoblotting (Figure $\mathbf{3 H}$ ), showing elevated Sox9 levels from day 6 onward. Gene expression of fibrillarin and dyskerin was measured in these samples. We detected a steady increase over-time of the mRNA expression of both fibrillarin and dyskerin, with a marked peak-expression at day 5 and 6 in differentiation, followed by maintenance of elevated fibrillarin and dyskerin expression as compared to non-differentiated $(t=0)$ ATDC5 (Figure 3E/F). The steady increase of fibrillarin and dyskerin expression was also detected at the protein level, albeit more evident for fibrillarin (Figure $\mathbf{3 H}$ ). These data indicate that during the course of chondrogenic differentiation, the snoRNA-guided 2'O-ribose methylation and pseudouridylation capacity is adapting through regulation of fibrillarin and dyskerin expression, with their pronounced peak-expression coinciding with the detectable start of chondrogenic differentiation (Sox9 and Col2a1 induction). 

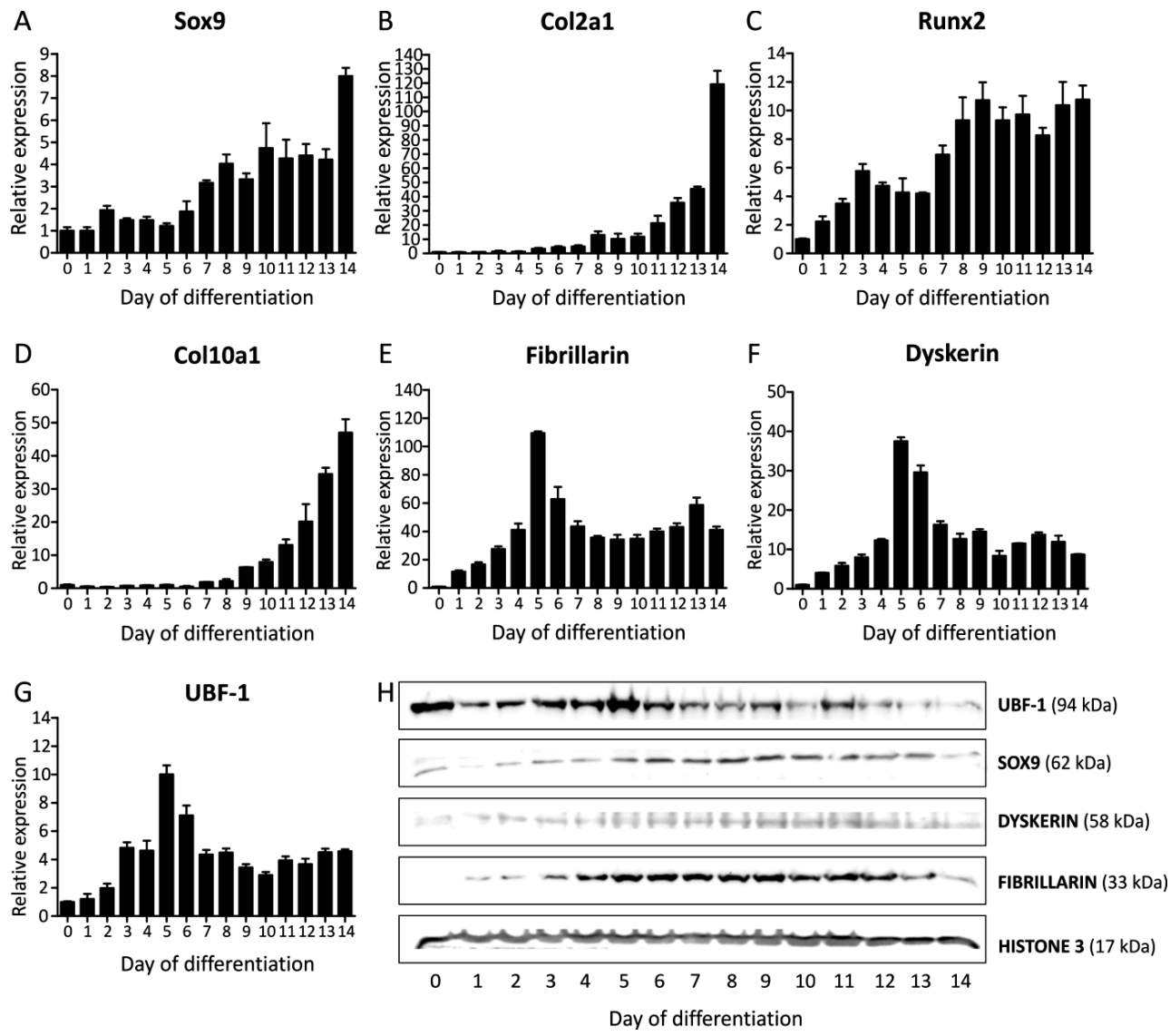

Figure 3. Fibrillarin, Dyskerin and UBF-1 expression adapts to the differentiation status of ATDC5 cells. ATDC5 cells were differentiated in the chondrogenic lineage for 14 days. A-D) Different stages of chondrogenic differentiation were confirmed by measuring gene expression of Sox9, Col2a1, Runx2 and Col10a1. H / SOX9) SOX9 protein expression was also determined. E-G) Gene expression of Fibrillarin, Dyskerin and UBF-1 was determined. Data (A-G) is depicted as fold induction relative to $t=0$. Data was normalized to $b$-actin and represents the average value of 4 biological replicates plus standard deviation. H) Protein expression of FIBRILLARIN, DYSKERIN and UBF-1 was detected on the same blot. HISTONE 3 was used as a housekeeper.

Taking furthermore into consideration that cartilaginous (collagenous) extra-cellular matrix synthesis becomes apparent in this timeframe in ATDC $5^{15,23}$, we questioned whether cellular protein translation capacity is regulated over-time in ATDC5 chondrogenic differentiation, to meet an increasing demand of extra-cellular matrix synthesis. Therefore we determined 


\section{Chapter 4}

whether rRNA levels are co-regulated during ATDC5 chondrogenic differentiation by measuring the expression of $18 \mathrm{~S}$ rRNA, 5.8S rRNA and 28S rRNA at 0, 7 and 14 days in differentiation. Data show that the expression of these rRNAs changes over-time, with significantly elevated levels specifically at day 7 , but not at day 14 in differentiation (Figure 4), compared to undifferentiated ( $\mathrm{t}=0$ ) ATDC5.

A

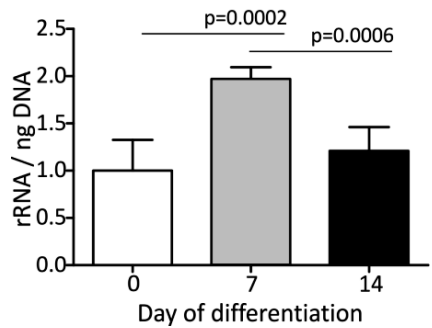

B

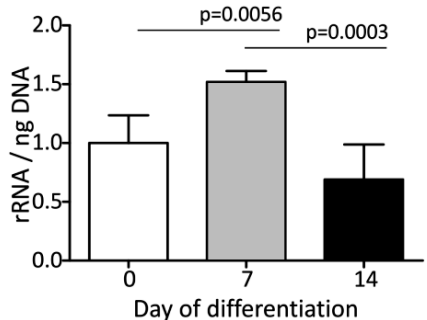

C

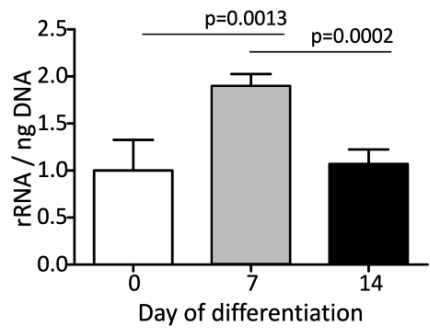

Figure 4. $18 \mathrm{~S}, 5.8 \mathrm{~S}$ and $28 \mathrm{~S}$ rRNA content per cell adapts to the differentiation status of ATDC5 cells. ATDC5 cells were differentiated in the chondrogenic lineage for 0,7 or 14 days. A-C) $18 \mathrm{~S}, 5.8 \mathrm{~S}$ and $28 \mathrm{~S}$ rRNA content per cell was quantified by calculating $18 \mathrm{~S}, 5.8 \mathrm{~S}$ and $28 \mathrm{~S}$ rRNA RTqPCR signals in six biological replicates (equal volumes of aqueous phase RNA were isolated and equal volumes of RNA were reverse transcribed to $C D N A$ ) relative to the DNA content in six biological replicates as quantified by SYBR green assay. Data (mean + standard deviation) is depicted as fold change relative to $t=0$. For statistical evaluation an independent samples $t$-test was performed between each consecutive time point using GraphPad Prism 5. p-values are indicated.

Total protein translation capacity was subsequently determined during the course of ATDC5 chondrogenic differentiation and we found that the protein translation capacity increased over-time during the course of ATDC5 chondrogenic differentiation, reaching its peak activity at day 7 in differentiation (Figure 5). At later time points in differentiation ATDC5 protein translation capacity remained induced, albeit at lower activity than at day 7 . 
A

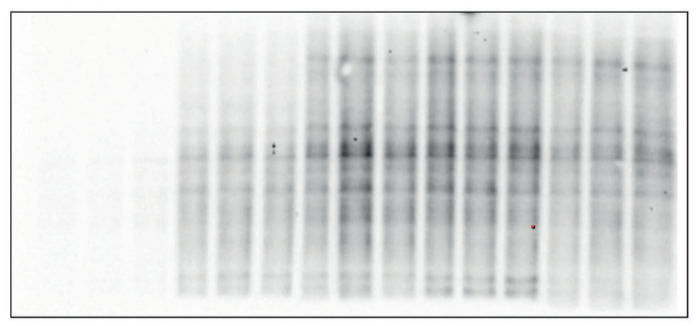

$\begin{array}{lllllllllllllll}0 & 0 & 0 & 3 & 3 & 3 & 7 & 7 & 7 & 10 & 10 & 10 & 14 & 14 & 14\end{array}$

Day of differentiation
B Puromycin incorporation

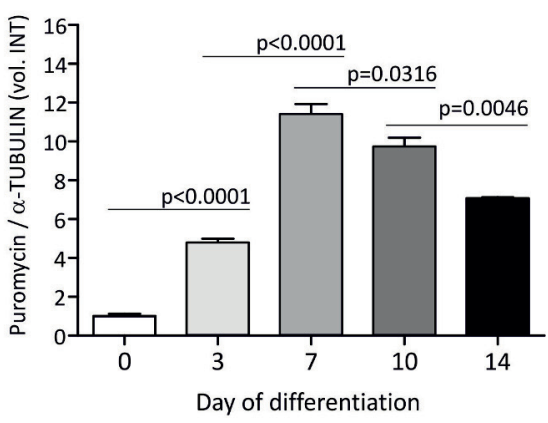

$\alpha$-TUBULIN (50 kDa)

\section{Figure 5. Active translation differs according to the differentiation status of ATDC5}

cells. To determine active protein synthesis, ATDC5 cells were labeled with puromycin 15 minutes prior to harvesting. Proteins were separated by gel electrophoresis and puromycin was detected. The experiment was performed with six biological replicates per time point. Here, three representative biological replicates per time point are shown (i.e. one of two Western blots). A) Whole lane puromycin signal (volume intensity; vol. INT) was quantified in all six biological replicates per time point and corrected for the quantified anti- $\alpha$-TUBULIN signal (housekeeper) (B) using Bio-Rad Image Lab Software 5.2.1. Data (mean + standard deviation) is depicted as fold change relative to $t=0$. For statistical evaluation an independent samples t-test was performed between each consecutive time point using GraphPad Prism 5. p-values are indicated.

The protein translation activity of differentiating ATDC5 was further investigated by polysome profiling. Sucrose gradient polysome profiling of cytoplasmic extracts of undifferentiated (day 0), and day 7 or day 14 differentiating ATDC5 revealed a strong increase in the monosomal ribosome fraction at day 7 in differentiation when compared to undifferentiated ATDC5 cells, while the polysomal distribution was largely unaltered. When progressing further into differentiation to day 14, the increased monosomal fraction observed at day 7 was reduced to a level that was still greater when compared to day 0 (Figure 6A). The polysomal fraction was again not altered, although a reduction in peak height can be observed between day 7 and 14 in differentiation. These dynamics observed in the monosomal fractions are in concert with the dynamics observed in total protein translation capacity over the course of differentiation (Figure 5). 
A
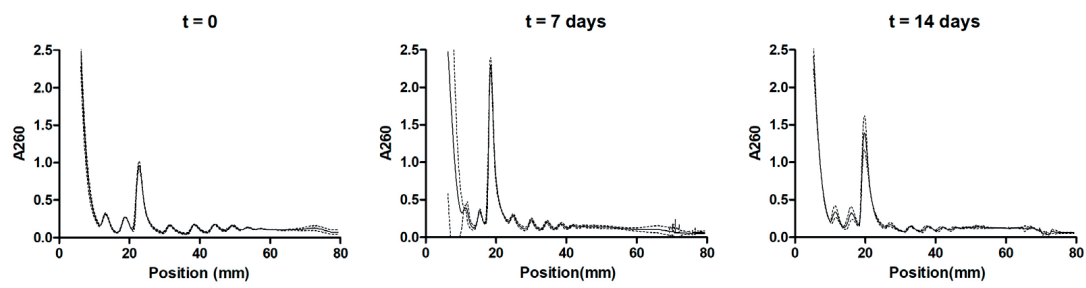

Figure 6. Polysome fractionation of differentiating ATDC5 cells. Cytoplasmic extracts were generated at day 0,7 and 14 of ATDC5 differentiation and equal amounts were ran on freshly prepared $10-50 \%$ sucrose gradients ( $n=4 /$ time point). After ultracentrifugation, sucrose gradients were fractionated with continuous recording of A260. Position ( $\mathrm{mm}$ ) indicates the position (depth) in the centrifugation tube with " 0 " representing the top and " 80 " the bottom of the centrifugation tube.

\section{Discussion}

Cell differentiation requires major changes in the cellular proteome to accommodate the cell specialization process ${ }^{24}$. Specifically, in the case of chondrogenic differentiation we expect that the de novo formation of the cartilaginous extracellular matrix demands a major proteomic effort. Consequently, ribosome biogenesis was expected to play an important role in chondrogenic differentiation. In keeping with this notion, rRNA levels were found to depend on the stage of chondrogenic differentiation. Specifically at day 7 in ATDC5 chondrogenic differentiation the rRNA expression levels were significantly higher, while rRNA levels were decreased again at day 14. This corresponded with increased protein translation and highest monosomal peaks in polysome profiling at day 7. These timings represent separate chondrogenic differentiation stages, with day 7 being early, highly proliferative ${ }^{25}$, and predominantly associated with extra-cellular matrix production, rich in type II collagen and aggrecan ${ }^{15,23}$. On the other hand, day 14 represents end-stage differentiation with expression of terminal differentiation markers like type $\mathrm{X}$ collagen and ALPL. One of the central transcription factors of RNA polymerase I-driven (RNAPI) 47S rDNA transcription is UBF-1. And, although RNAPI activity is regulated via UBF-1 at the posttranslational level (phosphorylation of UBF-1), the here observed dynamics in UBF-1 
expression during the course of chondrogenic differentiation strongly indicate that the increased rRNA levels at day 7 are, at least in part, the result of an increased transcription of the $47 \mathrm{~S}$ rDNA gene. In concert with the peak rRNA expression observed at day 7 in ATDC5 differentiation, total cellular translation capacity also reached its highest level at day 7 in differentiation, emphasizing that rRNA levels and translational capacity are functionally connected in the chondrogenic differentiation program. Chondrogenic differentiation in vivo and in vitro ${ }^{26}$ largely depends on IGF-1 signaling and indeed in vitro ATDC5 chondrogenic differentiation is also stimulated by insulin ${ }^{17}$. Insulin is one of the best-studied drivers of $47 \mathrm{~S}$ rDNA transcription and acts via UBF-1 dependent activation of the RNAPI machinery ${ }^{27}$. In addition, mTOR and 4E-BP activity are also induced by insulin and determine ribosome translational activity ${ }^{28}$. Together we therefore expect that during chondrogenic differentiation rRNA levels and protein translational activity are regulated by insulin signaling to meet the increased demand for cartilaginous extracellular matrix production and enabling the developing chondrocyte to translationally adapt to the cell specialization process.

Translational activity of the ribosome depends on many factors, with post-transcriptional modification of the rRNAs being pivotal in the basic biogenesis of the ribosome, supporting rRNA structural stability, ribosomal protein association and maturation of crucial ribosome functional regions, like the peptidyl transferase center (PTC) and the decoding center ${ }^{12,29}$. While hundreds of different rRNA post-transcriptional modifications are being guided by a great number of site-specific snoRNAs ${ }^{30}$, the core enzymatic activities responsible for these post-transcriptional modifications are fibrillarin ${ }^{31}$ (for 2'O-ribose methylation) and dyskerin (for pseudouridylation) ${ }^{32}$. Simultaneously with elevated rRNA levels at day 7 in ATDC5 chondrogenic differentiation, the expression of fibrillarin and dyskerin synchronously reached their highest levels as well. This observation may be explained by an increased de novo synthesized rRNA pool that requires modification. In addition, alterations in fibrillarin or dyskerin expression have been shown to provoke changes in ribosome translational characteristics. Knockdown of fibrillarin caused a reduction in global protein translation, with a specific reduction in IRES-dependent protein translation ${ }^{31}$, and p53-dependent expression of fibrillarin has been shown to regulate IRES-dependent translation and translational fidelity $^{33}$. Reduction of dyskerin levels also altered IRES-dependent translation, but with IRES-specific effects ${ }^{10,34}$. It is unknown whether these alterations in fibrillarin and dyskerin levels change translational characteristics via snoRNA-specific actions. Apart from the 


\section{Chapter 4}

apparent global increased need for rRNA post-transcriptional modification capacity, we established that the snoRNA expression landscape during ATDC5 chondrogenic differentiation changes, depending of the differentiation stage. This indicates that apart from the post-transcriptional maturation of a larger cellular rRNA pool, also the position of various rRNA post-transcriptional modifications may be adapting to the differentiation stage. There is limited literature reporting on the differential expression of snoRNAs in models for cell differentiation. In neural differentiation from embryonic stem cells, specific snoRNA species were found to be differentially expressed, depending on the differentiation stage ${ }^{9}$. In hematopoietic development, a great number of snoRNA was differentially expressed in a lineage specific pattern ${ }^{35}$. SnoRNA species were also expressed in a differentiation stagedependent manner during hepatic differentiation of induced pluripotent stem cells ${ }^{36}$. Apart from a number of non-canonical snoRNAs (like SNORD101, SNORD23, SNORA73 and others) that are involved in processes not involving rRNA post-transcriptional modification, the differentiation stage-depend dynamics in canonical snoRNA expression predicts a significant degree of rRNA post-transcriptional modification regulation during cell differentiation. A large number of snoRNAs with differentiation stage-dependent expression dynamics in snoRNA expression during ATDC5 chondrogenic differentiation are guiding posttranscriptional modification (PTM) of rRNA sites in the critical ribosome regions like the decoding center, the peptidyl transferase center and E-site. For example, SNORA40 (modifying helix 27 in the 18S rRNA decoding center in yeast ${ }^{37}$ ), SNORD46 (modifying helix 69 in $28 \mathrm{~S}$ rRNA $^{38,39}$ ) and SNORD36C and SNORA31 (modifying helix 68 in the 28S rRNA ribosome's E-site $\left.{ }^{40}\right)$. A recent seminal work showed regulation of rRNA 2'O-ribose methylation during mouse development and accompanying guide snoRNAs and highlights the relevance of ribosome heterogeneity during cell development ${ }^{41}$. In our ATDC5 chondrogenic differentiation model it remains to be determined whether the rRNA target sites of the differentially expressed snoRNAs are actually post-transcriptionally modified in a differentiation stage specific manner. With emerging technologies able to map rRNA posttranscriptional modifications ${ }^{42,43}$, we will be able to unveil the snoRNA-driven chondrogenic differentiation stage-specific post-transcriptional modification of rRNAs. Following identification of such differentiation stage-specific rRNA PTM-based ribosome heterogeneity, functional ribosome analyses (translational capacity, cap/IRES translation modus, stop-codon read-through activity, fidelity etc.) should be able to shed light on the 
role of these rRNA post-transcriptional modifications ${ }^{12}$ for the chondrogenic differentiation process.

In conclusion, our data show that chondrogenic differentiation is associated with significant regulation of mechanisms involving ribosome biogenesis and translation activity. Differentiation-phase specific expression of snoRNAs suggests that specific snoRNAs may modulate the chondrocyte's developing phenotype via an rRNA PTM-based ribosome heterogeneity mechanism, thereby potentially facilitating the observed dynamics in translational activity impacting the course of chondrogenic differentiation. Future work is expected to uncover the extent of ribosome heterogeneity and regulation in cellular differentiation and its potential implications for human disease. 


\section{References}

1 Ridanpaa, M. et al. Mutations in the RNA component of RNase MRP cause a pleiotropic human disease, cartilage-hair hypoplasia. Cell 104, 195-203, doi:10.1016/s0092-8674(01)00205-7 (2001).

2 Mason, P. J. \& Bessler, M. The genetics of dyskeratosis congenita. Cancer Genet 204, 635-645, doi:10.1016/j.cancergen.2011.11.002 (2011).

3 Valdez, B. C., Henning, D., So, R. B., Dixon, J. \& Dixon, M. J. The Treacher Collins syndrome (TCOF1) gene product is involved in ribosomal DNA gene transcription by interacting with upstream binding factor. Proc Natl Acad Sci U S A 101, 10709-10714, doi:10.1073/pnas.0402492101 (2004).

4 Ebert, B. L. et al. Identification of RPS14 as a 5q- syndrome gene by RNA interference screen. Nature 451, 335-339, doi:10.1038/nature06494 (2008).

5 Flygare, J. et al. Human RPS19, the gene mutated in Diamond-Blackfan anemia, encodes a ribosomal protein required for the maturation of $40 \mathrm{~S}$ ribosomal subunits. Blood 109, 980-986, doi:10.1182/blood-2006-07-038232 (2007).

6 Boocock, G. R. et al. Mutations in SBDS are associated with Shwachman-Diamond syndrome. Nat Genet 33, 97-101, doi:10.1038/ng1062 (2003).

7 Narla, A. \& Ebert, B. L. Ribosomopathies: human disorders of ribosome dysfunction. Blood 115, 3196-3205, doi:10.1182/blood-2009-10-178129 (2010).

8 Kronenberg, H. M. Developmental regulation of the growth plate. Nature 423, 332336, doi:10.1038/nature01657 (2003).

9 Skreka, K. et al. Identification of differentially expressed non-coding RNAs in embryonic stem cell neural differentiation. Nucleic Acids Res 40, 6001-6015, doi:10.1093/nar/gks311 (2012).

10 Jack, K. et al. rRNA pseudouridylation defects affect ribosomal ligand binding and translational fidelity from yeast to human cells. Mol Cell 44, 660-666, doi:10.1016/j.molcel.2011.09.017 (2011).

11 Lafontaine, D. L. Noncoding RNAs in eukaryotic ribosome biogenesis and function. Nat Struct Mol Biol 22, 11-19, doi:10.1038/nsmb.2939 (2015).

12 Sloan, K. E. et al. Tuning the ribosome: The influence of rRNA modification on eukaryotic ribosome biogenesis and function. RNA Biol 14, 1138-1152, doi:10.1080/15476286.2016.1259781 (2017).

13 Genuth, N. R. \& Barna, M. The Discovery of Ribosome Heterogeneity and Its Implications for Gene Regulation and Organismal Life. Mol Cell 71, 364-374, doi:10.1016/j.molcel.2018.07.018 (2018).

14 Kondrashov, N. et al. Ribosome-mediated specificity in Hox mRNA translation and vertebrate tissue patterning. Cell 145, 383-397, doi:10.1016/j.cell.2011.03.028 (2011). 
15 Steinbusch, M. M. F. et al. Expression of RMRP RNA is regulated in chondrocyte hypertrophy and determines chondrogenic differentiation. Sci Rep 7, 6440, doi:10.1038/s41598-017-06809-5 (2017).

16 Steinbusch, M. M. et al. Serum snoRNAs as biomarkers for joint ageing and post traumatic osteoarthritis. Sci Rep 7, 43558, doi:10.1038/srep43558 (2017).

17 Atsumi, T., Miwa, Y., Kimata, K. \& Ikawa, Y. A chondrogenic cell line derived from a differentiating culture of AT805 teratocarcinoma cells. Cell Differ Dev 30, 109-116 (1990).

18 Caron, M. M. et al. Activation of NF-kappaB/p65 facilitates early chondrogenic differentiation during endochondral ossification. PLoS One 7, e33467, doi:10.1371/journal.pone.0033467 (2012).

19 Robinson, M. D. \& Smyth, G. K. Moderated statistical tests for assessing differences in tag abundance. Bioinformatics 23, 2881-2887, doi:10.1093/bioinformatics/btm453 (2007).

20 Panda, A. C., Martindale, J. L. \& Gorospe, M. Polysome Fractionation to Analyze mRNA Distribution Profiles. Bio Protoc 7, doi:10.21769/BioProtoc.2126 (2017).

21 snoRNABase; a comprehensive database of human H/ACA and C/D box snoRNAs. Version 3. https://www-snorna.biotoul.fr/cherche.php.

22 Apollo Chemistry Gatech Ribovision Ribosome Visualization Suite. http://apollo.chemistry.gatech.edu/RibosomeGallery/.

23 Yao, Y. \& Wang, Y. ATDC5: an excellent in vitro model cell line for skeletal development. J Cell Biochem 114, 1223-1229, doi:10.1002/jcb.24467 (2013).

24 Sanchez, C. G. et al. Regulation of Ribosome Biogenesis and Protein Synthesis Controls Germline Stem Cell Differentiation. Cell Stem Cell 18, 276-290, doi:10.1016/j.stem.2015.11.004 (2016).

25 Spaapen, F. et al. The immediate early gene product EGR1 and polycomb group proteins interact in epigenetic programming during chondrogenesis. PLoS One 8, e58083, doi:10.1371/journal.pone.0058083 (2013).

26 Phornphutkul, C., Wu, K. Y. \& Gruppuso, P. A. The role of insulin in chondrogenesis. Mol Cell Endocrinol 249, 107-115, doi:10.1016/j.mce.2006.02.002 (2006).

27 Wu, A., Tu, X., Prisco, M. \& Baserga, R. Regulation of upstream binding factor 1 activity by insulin-like growth factor I receptor signaling. J Biol Chem 280, 2863-2872, doi:10.1074/jbc.M406138200 (2005).

28 Wang, L., Rhodes, C. J. \& Lawrence, J. C., Jr. Activation of mammalian target of rapamycin (mTOR) by insulin is associated with stimulation of 4EBP1 binding to dimeric mTOR complex 1. J Biol Chem 281, 24293-24303, doi:10.1074/jbc.M603566200 (2006).

29 Taoka, M. et al. Landscape of the complete RNA chemical modifications in the human $80 S$ ribosome. Nucleic Acids Res 46, 9289-9298, doi:10.1093/nar/gky811 (2018). 
30 Dupuis-Sandoval, F., Poirier, M. \& Scott, M. S. The emerging landscape of small nucleolar RNAs in cell biology. Wiley Interdiscip Rev RNA 6, 381-397, doi:10.1002/wrna.1284 (2015).

31 Erales, J. et al. Evidence for rRNA 2'-O-methylation plasticity: Control of intrinsic translational capabilities of human ribosomes. Proc Natl Acad Sci U S A 114, 1293412939, doi:10.1073/pnas.1707674114 (2017).

32 De Zoysa, M. D. \& Yu, Y. T. Posttranscriptional RNA Pseudouridylation. Enzymes 41, 151-167, doi:10.1016/bs.enz.2017.02.001 (2017).

33 Marcel, V. et al. p53 acts as a safeguard of translational control by regulating fibrillarin and rRNA methylation in cancer. Cancer Cell 24, 318-330, doi:10.1016/j.ccr.2013.08.013 (2013).

34 Penzo, M. et al. Human ribosomes from cells with reduced dyskerin levels are intrinsically altered in translation. FASEB J 29, 3472-3482, doi:10.1096/fj.15-270991 (2015).

35 Warner, W. A. et al. Expression profiling of snoRNAs in normal hematopoiesis and AML. Blood Adv 2, 151-163, doi:10.1182/bloodadvances.2017006668 (2018).

36 Skrzypczyk, A. et al. Noncoding RNA Transcripts during Differentiation of Induced Pluripotent Stem Cells into Hepatocytes. Stem Cells Int 2018, 5692840, doi:10.1155/2018/5692840 (2018).

37 Velichutina, I. V. et al. Mutations in helix 27 of the yeast Saccharomyces cerevisiae $18 \mathrm{~S}$ rRNA affect the function of the decoding center of the ribosome. RNA 6, 11741184 (2000).

38 Sergiev, P. V., Bogdanov, A. A. \& Dontsova, O. A. Ribosomal RNA guanine-(N2)methyltransferases and their targets. Nucleic Acids Res 35, 2295-2301, doi:10.1093/nar/gkm104 (2007).

39 Liiv, A., Karitkina, D., Maivali, U. \& Remme, J. Analysis of the function of E. coli 235 rRNA helix-loop 69 by mutagenesis. BMC Mol Biol 6, 18, doi:10.1186/1471-2199-6-18 (2005).

40 Greber, B. J. et al. The complete structure of the large subunit of the mammalian mitochondrial ribosome. Nature 515, 283-286, doi:10.1038/nature13895 (2014).

41 Hebras, J., Krogh, N., Marty, V., Nielsen, H. \& Cavaille, J. Developmental changes of rRNA ribose methylations in the mouse. RNA Biol 17, 150-164, doi:10.1080/15476286.2019.1670598 (2020).

42 Marchand, V., Blanloeil-Oillo, F., Helm, M. \& Motorin, Y. Illumina-based RiboMethSeq approach for mapping of 2'-O-Me residues in RNA. Nucleic Acids Res 44, e135, doi:10.1093/nar/gkw547 (2016).

43 Schwartz, S. et al. Transcriptome-wide mapping reveals widespread dynamicregulated pseudouridylation of ncRNA and mRNA. Cell 159, 148-162, doi:10.1016/j.cell.2014.08.028 (2014). 
Adaptation of the protein translational apparatus during ATDC5 chondrogenic differentiation 
1 Department of Orthopedic Surgery, Caphri School for Public Health and Primary Care, Maastricht University Medical Center, Maastricht, the Netherlands

2 Centre for Genomic Research, Institute of Integrative Biology, Biosciences Building, Crown Street, University of Liverpool, Liverpool L69 7ZB, UK

3 Institute of Ageing and Chronic Disease, University of Liverpool, Apex Building, 6 West Derby Street, Liverpool, L7 9TX, UK

${ }^{4}$ Musculoskeletal Research Group, Newcastle University, Newcastle upon Tyne NE2 4HH, UK 


\section{Chapter 5}

\section{Serum snoRNAs as biomarkers for joint ageing and post traumatic osteoarthritis}

Mandy MF Steinbusch', Yongxiang Fang ${ }^{2}$, Peter Milner ${ }^{3}$, Peter D Clegg ${ }^{3}$, David Young ${ }^{4}$, Tim JM Welting ${ }^{1}$, Mandy J Peffers ${ }^{3}$

Published in Nature Scientific Reports, Sci Rep 7 (2017), 43558, doi: 10.1038/srep43558 


\section{Abstract}

The development of effective treatments for the age-related disease osteoarthritis and the ability to predict disease progression has been hampered by the lack of biomarkers able to demonstrate the course of the disease. Profiling the expression patterns of small nucleolar RNAs (snoRNAs) in joint ageing and OA may provide diagnostic biomarkers and therapeutic targets. This study determined expression patterns of snoRNAs in joint ageing and OA and examined them as potential biomarkers. Using SnoRNASeq and real-time quantitative PCR (qRT-PCR) we demonstrate snoRNA expression levels in murine ageing and OA joints and serum for the first time. SnoRNASeq identified differential expression (DE) of 6 snoRNAs in young versus old joints and 5 snoRNAs in old sham versus old experimental osteoarthritic joints. In serum we found differential presence of 27 snoRNAs in young versus old serum and 18 snoRNAs in old sham versus old experimental osteoarthritic serum. Confirmatory qRT-PCR analysis demonstrated good correlation with SnoRNASeq findings. Profiling the expression patterns of snoRNAs is the initial step in determining their functional significance in ageing and osteoarthritis, and provides potential diagnostic biomarkers and therapeutic targets. Our results establish snoRNAs as novel markers of musculoskeletal ageing and osteoarthritis. 


\section{Introduction}

Osteoarthritis (OA) is an age-related musculoskeletal disease and a common cause of chronic disability worldwide ${ }^{1}$. In addition it is a significant contributor to both individual and socioeconomic burden and the number of disability adapted life years globally ${ }^{2}$. If the deterioration in musculoskeletal health and development of $\mathrm{OA}$ can be identified and treated early serious life impairment may be abrogated. Ageing is the time-dependent reduction of functional capacity and stress resistance, associated with an increased risk of morbidity and mortality. The joint and its articular cartilage is particularly affected by ageing $^{3}$. There is evidence that the rate of ageing, that is the 'biological age', differs significantly between individuals' actual age in years (i.e. the 'chronological age'). Defining markers of joint ageing may enable a prediction of the risk of onset of $O A$, enabling early intervention. $\mathrm{OA}$ is characterised by a non-symptomatic, pre-radiographical phase that if identified would allow earlier diagnosis. However radiographic changes are only evident later in disease progression. Magnetic resonance imaging techniques have been developed for early-stage evaluation of cartilage damage in OA but are expensive and contraindicated in some individuals.

The development of effective treatments for $\mathrm{OA}$ and the ability to predict disease progression has been hampered by the lack of substantive biomarkers, able to demonstrate pathological disturbances preceding identifiable tissue alterations. Others have attempted to identify products of tissue turnover in serum and synovial fluid (reviewed $\left.{ }^{4}\right)$. This has been challenging due to patient and disease heterogeneity and dilution effects either by tissue fluids or with similar products from other joints or diseases. In addition, the variability of antibody assays has been problematic.

SnoRNAs are a class of evolutionary conserved non-coding small guide RNAs of which the majority direct the chemical modification of other RNA substrates, including ribosomal RNAs and spliceosomal RNAs. In addition, some snoRNAs are involved in the regulation of alternative splicing and post-transcriptional modification of mRNA, whilst others exhibit 


\section{Chapter 5}

miR-like activity ${ }^{5}$. Aberrant expression of snoRNAs has been associated with disease development ${ }^{5}$ such as lung tumorigenesis ${ }^{6}$.

Emerging evidence shows that there is an increased level of circulating RNAs in the serum of cancer patients ${ }^{7}$. Circulating microRNAs (miRs) have been extensively described as biomarkers for diseases like pancreatic/breast cancer ${ }^{8,9}$, Alzheimer's disease ${ }^{10}$ and inflammatory diseases like asthma, inflammatory bowel disease and rheumatoid arthiritis ${ }^{11}$, but with the recent discovery of stable ${ }^{12}$ snoRNAs in serum, interest in their potential as circulating biomarkers of cancers (reviewed ${ }^{5}$ ) has been stimulated. We have previously identified dysregulation of a defined set of snoRNAs in cartilage ${ }^{13}$ and tendon ${ }^{14}$ ageing and $\mathrm{OA}^{15}$ and in man, snoRNA SNORD38 and SNORD48 were identified as potential non-agedependant serum biomarkers for OA progression following cruciate ligament injury ${ }^{12}$.

Expression profiling of snoRNAs in ageing and OA may help in determining their functional significance in the development and progression of disease and provide much needed diagnostic biomarkers for ageing and OA development. This study compared serum and joint snoRNA expression in ageing and OA from knee joint tissues from young and old adult mice and old mice using a traumatic in vivo model of OA. Because OA involves the whole joint as an organ; we undertook our analysis on whole mouse joints, which included cartilage, meniscus, subchondral bone, and joint capsule with synovium.

\section{Materials and Methods}

All reagents were from Thermo-Fisher-Scientific, unless stated.

\section{Animals}

C57BL6/J male mice were used for the study. For SnoRNASeq old mice were 18 months old $(n=6)$, young 8 months old $(n=6)^{16}$ and mice used for destabilisation of the medial meniscus (DMM) 24 months old (sham $n=3 ; D M M n=6$ ). Mice were group housed in individually ventilated cages at a 12 hour light/dark cycle, with ad libitum access to food and water. 
Experimental animal protocols were performed in accordance with the guidelines of the Animals (Scientific Procedures) Act 1986 following ethical review. Animal usage and protocols for this study was approved by the University of Liverpool Animal Welfare Committee.

\section{Surgical induction of OA by DMM in mice}

DMM surgery was perform as previously reported ${ }^{17}$. Briefly, under anaesthesia a $3 \mathrm{~mm}$ skin incision was made over the medial aspect of the patellar ligament through the joint capsule

into the femorotibial joint of the left knee. The medial meniscotibial ligament was transected to destabilise the cranial pole of the medial meniscus from the anterior tibial plateau. In sham operated mice the medial meniscotibial ligament was visualised but not transected. Mice were sacrificed 8 weeks post-surgery.

\section{Joint and serum collection for SnoRNASeq}

Following euthanasia, knee joints were collected from young, old, DMM ( $n=6$ each group) and sham $n=3$ for SnoRNASeq. Joints were harvested free of soft tissues at $7 \mathrm{~mm}$ from the joint into RNALater. Serum was collected using cardiac puncture. One old serum sample was not processed further due to extensive haemolysis.

\section{OARSI scoring of histological sections of mouse knee joints}

For histology, as the total knee joint was used in the study for RNA extraction, joints were collected (into $4 \%$ paraformaldehyde) from additional equivalent aged and treated young $(n=8)$, old $(n=4)$; sham $(n=5)$; and DMM $(n=6)$ mice in order to evaluate the extent of OA. The procedure, surgeon and duration of the studies were identical. Knees were decalcified in $0.5 \mathrm{M}$ ethylenediaminetetraacetic acid $(\mathrm{pH} 7.4)$ for 4 weeks at $4^{\circ} \mathrm{C}$ and coronally embedded in paraffin. Sectioning, Safranin-O Fast-Green staining and histological scoring (defined as the severity and extent of OA) was undertaken on a scale from 1 to 6 by two 


\section{Chapter 5}

blinded independent observers using the OARSI histopathology initiative ${ }^{18}$. All four quadrants of the section (medial tibial plateau, lateral tibial plateau, medial femoral condyle, lateral femoral condyle) were scored individually and added for each histological section. For statistical analyses mean summed score values of joints of 3-5 section per knee joint at 4 depths throughout the joint was determined (thus a maximum score of 24 was possible). Inter-observer variability was calculated using Cohen's Kappa statistics using an online software tool: (http://www.statstodo.com/CohenKappa).

\section{RNA isolation, RNA-Seq analysis, cDNA library preparation and sequencing}

Total RNA was isolated from equal weights of joints and $500 \mu \mathrm{l}$ serum using miRNeasy or RNeasy Serum kits respectively with DNase treatment (all Qiagen, Crawley, UK) to remove residual gDNA. Total RNA integrity (RIN) was confirmed using the Agilent 2100 Bioanalyzer (Agilent Technologies, Santa Clara, USA). Ribosomal RNA was depleted using the Ribo-Zero ${ }^{\mathrm{TM}}$ rRNA Removal Kit (Epicentre, Madison, USA). From 41 samples 100 ng of rRNA-depleted RNA was submitted for library preparation using NEB small RNA library kit (New England Biolabs (NEB), Ipswich, USA). To reduce workflow bias we used tobacco acid pyrophosphatase (Epicentre, Madison, USA) to remove potential 5' caps found on some snoRNAs. Samples were amplified for 15 cycles, mixed into 3 pools, and size selected. The size-selected material was purified with Ampure beads (Agencourt, Beckman-Coulter, HighWycombe, UK). SnoRNA sequencing was undertaken on the Illumina HiSeq 2000 platform (Illumina, San Diego, USA) using 100 base paired-end reads.

\section{SnoRNASeq data analysis}

Sequence data measured from 5 lanes of an Illumina HiSeq2000 were processed through a number of steps to obtain snoRNA expression values. The processes include basecalling and de-multiplexing of indexed reads using CASAVA version 1.8.2 ${ }^{19}$; adapter and quality trimming using Cutadapt version 1.2.1 $1^{20}$ and Sickle version 1.200 to obtain fastq files of trimmed reads; aligning reads to Ensembl GRCm38.77 mouse genome reference sequences 170 
which contains 1,555 annotated snoRNA features using Bowtie $2^{21}$ version 2.0.10 with option "very-sensitive-local"; counting aligned reads against snoRNA features using THSeq-count. The count values were used as snoRNA expression measurements for the DE analysis.

$\mathrm{DE}$ analysis was performed in $\mathrm{R}$ environment using package edge $\mathrm{R}^{22}$. The processes and technical details of the analysis include: assessing data variation and detecting outlier samples through comparing variations of within and between sample groups using principle component analysis (PCA; 3-D PCA plots were generated using R function in package plot3D) and correlation analysis; handling library size variation respectively for joint samples and serum samples through data normalisation; formulating data variation using negative binomial distributions; modelling data using a generalised linear model; computing $\log _{2}$ Fold Change $(\log F C)$ values for required contrasts based on model fitting results through contrast fitting approach, assigning P-values to logFC values by $L^{23}$ testing; dealing with the effects of multiple tests using FDR approach to obtain FDR adjusted P-values; and defining significantly DE snoRNAs as those with FDR-adjusted $p$-value $<5 \%$. Sequence data have been submitted to National Centre for Biotechnology Information Gene Expression Omnibus (NCBI GEO); E-MTAB-4878.

\section{RNA isolation, poly(A) cDNA synthesis and snoRNA qRT-PCR}

qRT-PCR of snoRNAs was performed ${ }^{24}$. Total RNA was isolated using the mirVana kit. Isolated RNA samples were polyadenylated at $37^{\circ} \mathrm{C}$ for 60 minutes in a $50 \mu \mathrm{L}$ reaction volume containing $1 \mu \mathrm{g}$ RNA and $1.5 \mathrm{U}$ poly(A) polymerase (NEB, Ipswich, USA). $500 \mu \mathrm{L}$ lysis binding buffer was added. Then, an equal volume of acid-phenol:chloroform was added, vortexed and samples were centrifuged for 10 minutes and the aqueous phase removed. The poly(A)-tailed total RNA was extracted using the filter cartridge provided by the mirVana kit. To generate poly(A) cDNA, $500 \mathrm{ng}$ poly(A)-tailed RNA and $250 \mathrm{ng}$ RTQ primer (Eurogentec, Seraing, Belgium) (Table 1) were mixed in a $26 \mu \mathrm{L}$ reaction volume, incubated at $65^{\circ} \mathrm{C}$ for 10 minutes and annealed at $4^{\circ} \mathrm{C}$ for 20 minutes. Reverse transcription was performed with $200 \mathrm{U}$ M-MLV reverse transcriptase, $20 \mathrm{U}$ RNAsin (both Promega, Southampton, UK), $2 \mu \mathrm{L}$ dNTP mix (10 mM each; Eurogentec, Seraing, Belgium) and $8 \mu \mathrm{L} 5 \mathrm{x}$ 
M-MLV buffer (Promega, Sothampton, USA) in a total reaction volume of $40 \mu \mathrm{L}$ at $50^{\circ} \mathrm{C}$ for 60 minutes. The reverse transcriptase was inactivated at $70^{\circ} \mathrm{C}$ for 15 minutes. Finally, $1.5 \mathrm{U}$ of RNAse $\mathrm{H}$ (NEB, Ipswich, USA) was added to remove small RNAs. A snoRNA-specific forward primer and a universal reverse primer (RTQ-UNIr, matched to the Tm of each individual snoRNA) were used for the amplification of each snoRNA target (all Eurogentec, Seraing, Belgium) (Table 1). For each cDNA sample a mix was prepared with Mesagreen qPCR Mastermix Plus for SYBR Green (Eurogentec, Seraing, Belgium) and $300 \mathrm{nM}$ forward and reverse oligonucleotides. An ABI-7300 Detection System was used for amplification using the following protocol: denaturation at $95^{\circ} \mathrm{C}$ for 5 minutes, followed by 50 cycles of DNA amplification (15 seconds $95^{\circ} \mathrm{C}$ and 45 seconds annealing at $62-68^{\circ} \mathrm{C}$ ). The annealing temperature was optimized for each snoRNAs target. Serially diluted standard curves were utilized to quantify snoRNA expression and data was normalized to a validated housekeeping snoRNA (joint: U2, young-old serum: SNORD85, old sham-old DMM serum and equine serum: U6).

Table 1. Oligonucleotides sequences used in qRT-PCR

\begin{tabular}{|l|l|c|}
\hline Name & Sequence $\left(\mathbf{5}^{\prime}\right.$-3') & Tm ( $\left.^{\circ} \mathbf{C}\right)$ \\
\hline RTQ primer poly(A) & CGAATTCTAGAGCTCGAGGCAGGCGACATGGCT & 75 \\
GGCTAGTTAAGCTTGGTACCGAGCTCGGATCCA & \\
\hline CTAGTCCTTTTTTTTTTTTTTTTTTTTTTTTTVN & \\
\hline Snora30 & CATGAGACAAGCCGTTATATAGGC & 50 \\
\hline Snora31 & TGTACCAGTGGCAGCTGTTACTC & 50 \\
\hline Snora64 & CTTTGTGGCAGTTCAGATTGAATTAG & 50 \\
\hline Snora73 & GTGGCCTCTCTTGCCTAGAG & 65 \\
\hline Snord46 & ACAGTGACTGAGGAGGCAAAC & 50 \\
\hline Snord85 & AATGCAAGGACTTGTCATAGTTACAC & 50 \\
\hline Snord88 & ACCTTTGACCAGAGGTCGATGATGAG & 50 \\
\hline Snord116 & TGTACCGCCACTCTCATCGG & 50 \\
\hline U2 & TGGTATTGCAGTACCTCCAGGAACG & 65 \\
\hline U3 & AGTGAGAGGGAGAGAACGCGGTC & 55 \\
\hline U6 & GATGACACGCAAATTCGTGAAGCGTTC & 55 \\
\hline RTQ-UNIr-50 & AATTCTAGAGCTCGAGGCAGG & 50 \\
\hline
\end{tabular}




\begin{tabular}{|l|l|c|}
\hline Name & Sequence $\left(\mathbf{5}^{\prime} \mathbf{- 3}^{\mathbf{\prime}}\right)$ & $\mathbf{T m}\left({ }^{\circ} \mathrm{C}\right)$ \\
\hline RTQ-UNIr-55 & CGAATTCTAGAGCTCGAGGCAGG & 55 \\
\hline RTQ-UNIr-65 & CTAGAGCTCGAGGCAGGCGACATGGCTGGC & 65 \\
\hline
\end{tabular}

\section{Validation of SNORD116 as a marker of OA in equine serum}

We determined the reproducibility of the expression of SNORD116 in OA serum of another species. There are well-published studies on the application of metacarpophalangeal (MCP) joint OA changes in the horse, a joint with similarities to the human knee joint ${ }^{25}$. We studied

equine serum from normal and MCP OA horses collected from eight normal (mean age \pm standard deviation $5.3 \pm 2.1$ years) and four $\mathrm{OA}(7.5 \pm 1.0$ years) castrated male thoroughbred horses at post-mortem. Samples were collected under the regulations of the Hong Kong Jockey Club with owner consent and stored at $-80^{\circ} \mathrm{C}$. OA diagnosis was based on histological (modified Mankin) ${ }^{26}$ and synovitis scoring 27 of MCP joint tissues.

\section{Statistical analysis}

For statistical evaluation of histological scoring non-parametric Mann-Whitney-U test was used. Inter-observer agreement of histological scoring systems was calculated using Cohen's kappa coefficient (www.statstodo.com/CohenKappa_Pgm.phpl). qRT-PCR data was logtransformed prior to statistical evaluation with an independent samples t-test. Statistical evaluation was performed between young and old or old sham versus old DMM using GraphPad Prism 5 (San Diego); p-values are indicated. 


\section{Results}

\section{OARSI scoring of joints}

OARSI scoring of joints (mean $\pm 95 \% \mathrm{Cl}$ ) for young and old were $0.5 \pm 0.3$ and $2.8 \pm 2.7(p=0.01)$, and old sham and old DMM were $1.25 \pm 1.1$ and $6.5 \pm 0.7 \quad(p<0.001)$, respectively. Mice exhibited typical histological features of OA in the DMM knees. Cohen's Kappa statistic was 0.4 indicating a fair agreement. Representative histological images and OARSI scoring are in Fig. 1.

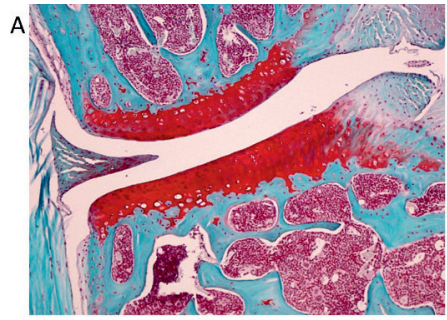

Young

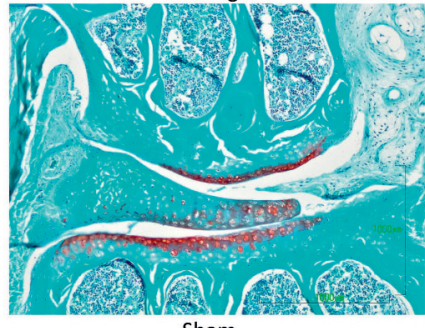

Sham

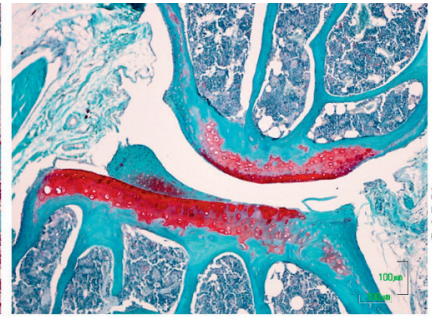

Old

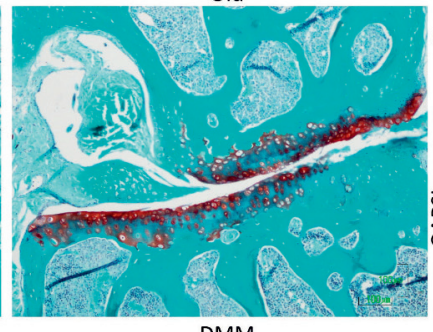

DMM

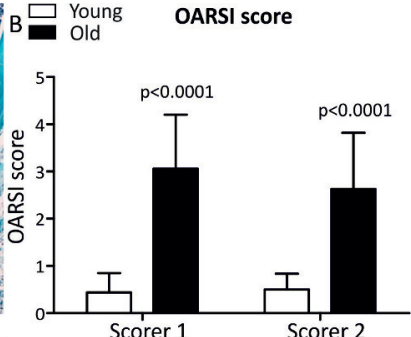

OARSI score

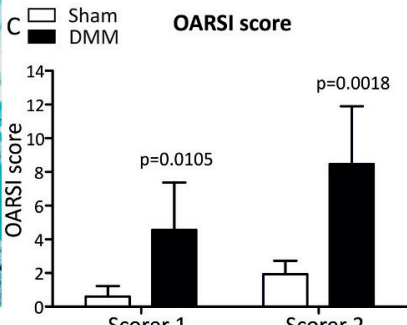

Scorer 1

Figure 1. Histological changes of in the mouse knee showing the medial femoral condyle (above) and medial tibial plateau (below). A. Safranin $O$ with Fast-Green counterstain. Scale bar, $100 \mu \mathrm{m}$. Red indicates proteoglycan. B/C. Assessment of osteoarthritis development was evaluated by OARSI scores; young $(n=8)$ vs. old $(n=4)(B)$; sham $(n=5)$ vs. DMM $(n=6)(C)$. Data represents the mean $+95 \%$ confidence interval $(\mathrm{Cl})$ for each scorer. For statistical evaluation an independent samples t-test was performed using GraphPad Prism 5 (San Diego); pvalues are indicated. 


\section{Preliminary analysis SnoRNASeq}

To identify DE of snoRNAs in mouse joints and serum in response to age and OA 41 cDNA libraries representing old and young joints (old=HJO; young=HJY) and serum (old=HSO; young =HSY), and old sham and old DMM joints and serum (sham joint=DJC, DMM joint=DJM, sham serum=DSC and DMM serum=DSM) were constructed and subjected to

Illumina deep sequencing. Summaries of raw, trimmed reads and sequencing alignment to mouse snoRNAs are in Supplementary files 1 and 2 respectively. Reads mapping percentages for joint libraries were between 12 29\%, and for serum libraries $0.03 \sim 0.9 \%$. Between $42 \sim 53 \%$ of the 1555 mouse snoRNA reference sequences were aligned for joints and $16 \sim 26 \%$ for serum.

\section{Identification of DE snoRNAs using SnoRNASeq}

The 3-D PCA plot (Fig. 2A) shows that the joint samples and serum samples are clearly separated by the 1 st component, which explains $93.02 \%$ of the data variation. For serum samples, the group sham and the group DMM scatter separately on the 2nd component, furthermore, based on the 3rd component a clear separation between serum samples of young-healthy and samples of old-healthy exists. For joint samples, there is also a separation between DMM and sham samples, though the separation is not as clear as shown for serum samples. In addition, 5 DMM joint samples scatter far away from other joint samples on the 3rd component. Therefore, it can be expected that disease response small RNA can be detected from this data set. The heat map of hierarchical clusters of correlations among samples (Fig. 2B) depicts that the joint and serum groups of samples are very different in snoRNA expression. In addition, joint samples correlated to each other much more closely than serum samples confirming the phenomena revealed by the PCA analysis shown in Fig. 2A. This indicates that major disease responses in the data were contributed from serum samples. A read length distribution graph was generated to highlight the constitutional difference of non-coding RNAs in joint and serum samples at 100 bp or below (Fig. 2C). For this one serum and one joint sample with approximately the same library size were chosen. The plots of the read length distribution for joint and serum 


\section{Chapter 5}

samples reveal that a peak value of frequency for read length in joint samples is $22 \mathrm{bp}$ (vertical, dotted black line). Therefore the corresponding reads are generally well annotated miRNAs. Another peak is noted at around $100 \mathrm{bp}$, which is consistent with non-coding RNAs whose length is a hundred bp and over. In contrast, a peak is presented at $30 \mathrm{bp}$ long for serum samples (vertical, dotted red line). More than $50 \%$ of the reads are $30 \mathrm{bp}$ long in the serum library (data not shown). In contrast, the joint library has just $1.14 \%$ of reads that are $30 \mathrm{bp}$ long (data not shown). Such reads may come from piwi-interacting RNAs (piRNAs). There were 498-646 snoRNAs expressed in serum samples and 1068-1286 in joint samples. The DE snoRNAs between contrasts are in Table 2. These included 6 snoRNAs in young versus old joints, 5 snoRNAs in old sham versus old DMM joints. In serum we identified DE of 27 snoRNAs in young versus old serum and 18 snoRNAs in old sham versus old DMM serum. 
A

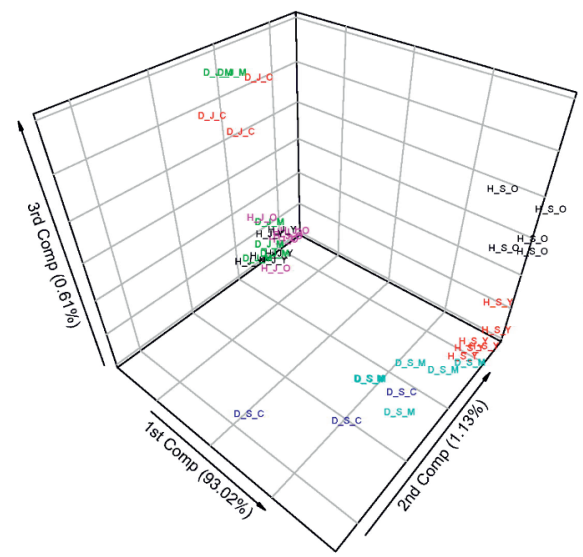

B
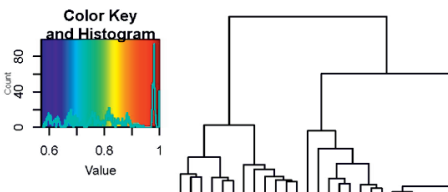

Group classification:

H_J_Y: Joint - young

D__C: Joint - old sham

D. M: Joint- old DMM

H_S_Y: Serum - young

H_S_O: Serum - old

D_S_C: Serum - old sham

D_S_M: Serum - old DMM
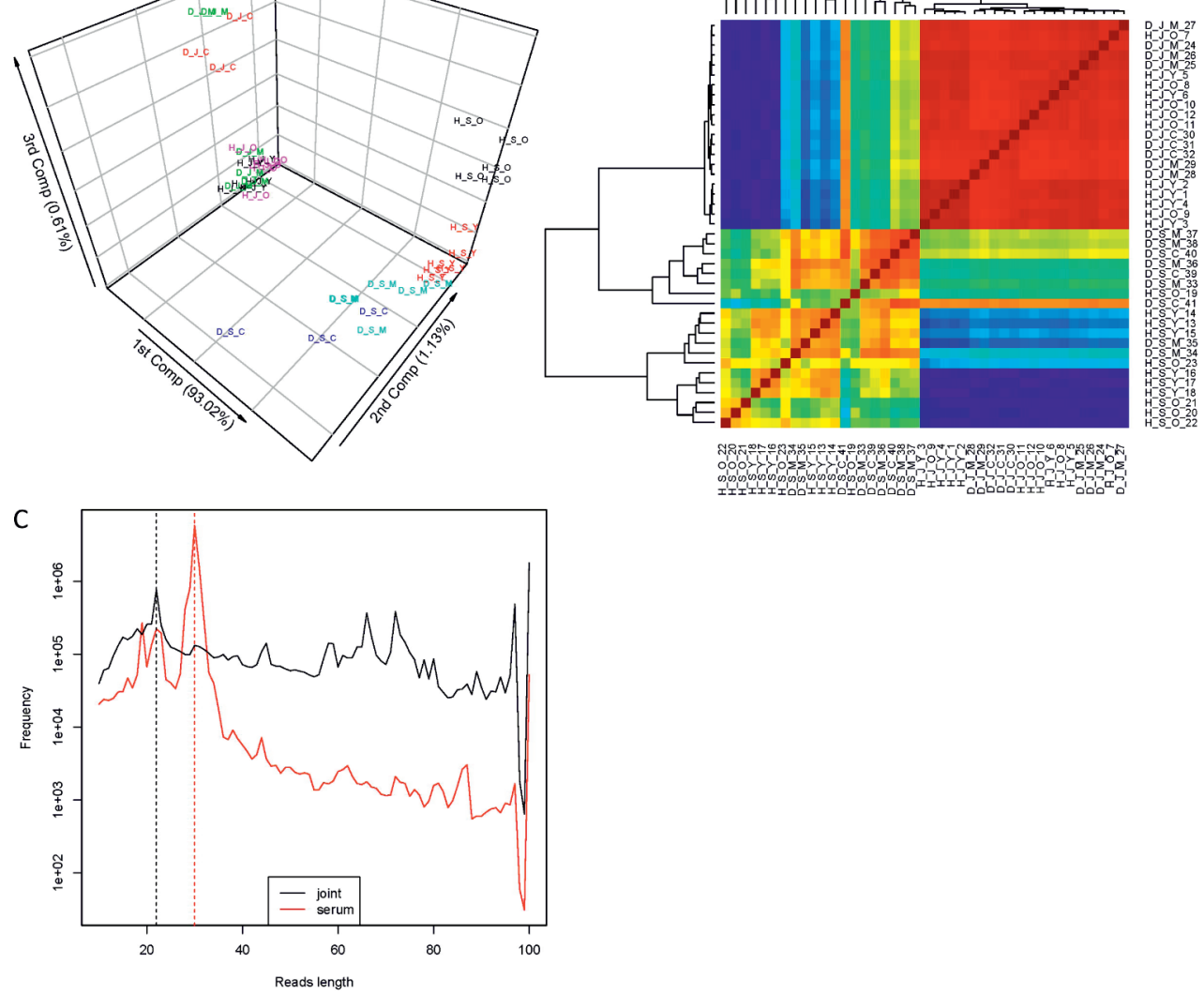

Figure 2. Variation data between the expressions for 41 samples. A. A 3-D PCA plot of the first three components from principal component analysis of logarithm-transformed small RNA abundance data. Variance (\%) associated with each principle component is depicted on the respective axis. Abbreviations; Joint: young healthy (HJY; black), old healthy (HJO; red), old sham (DJC; magenta), old DMM (DJM; light blue). Serum: young healthy (HSY; green), old healthy (HSO; dark blue), old sham (DSC; grey), old DMM (DSM; yellow). B. The heat map of hierarchical clusters of correlations among samples. Pearson's correlation coefficients were computed using logarithm transformed small RNA expression data from all known snoRNAs that were detected. C. The plots of read length distribution for two representative joint and serum samples. 
Chapter 5

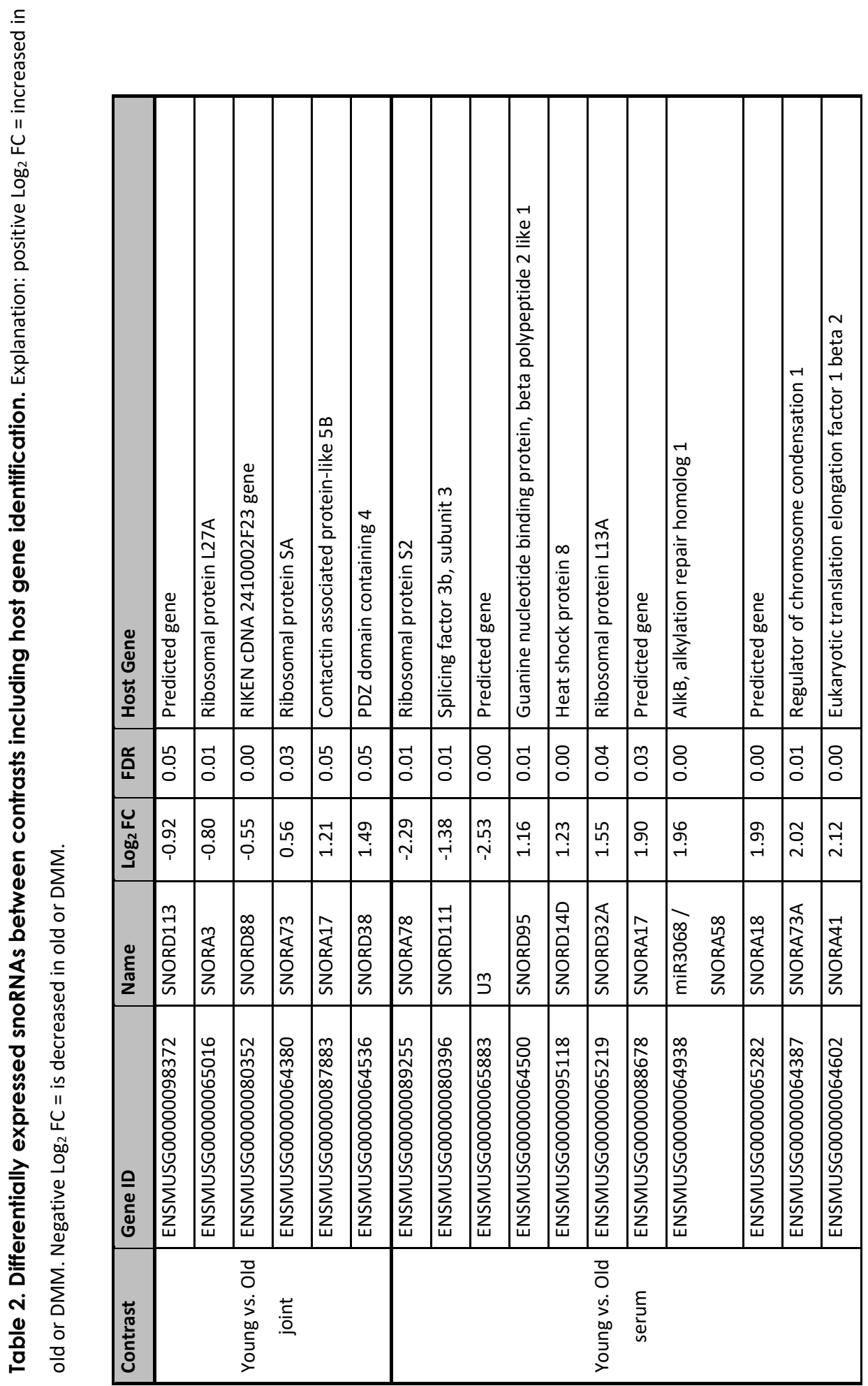




\begin{tabular}{|c|c|c|c|c|c|c|c|c|c|c|c|c|c|c|c|c|c|c|c|c|}
\hline 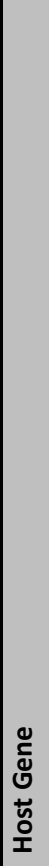 & 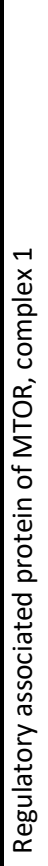 & 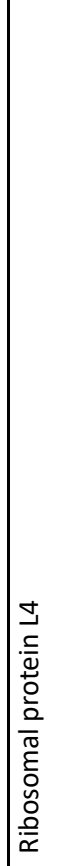 & 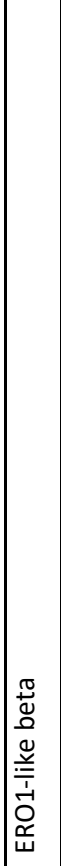 & 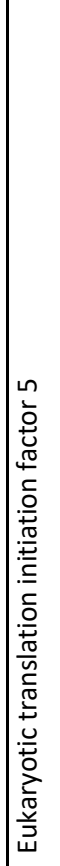 & 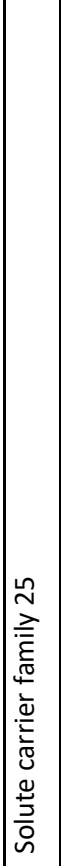 & 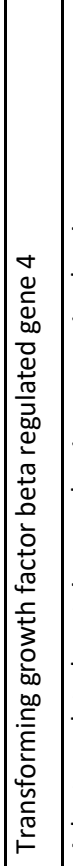 & 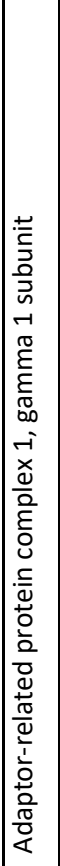 & 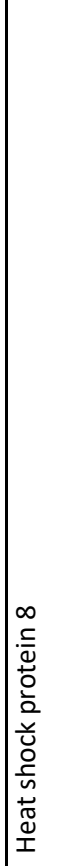 & 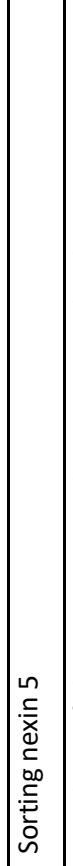 & 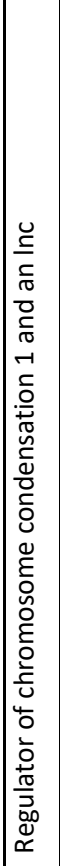 & $\begin{array}{l}\frac{. \subseteq}{\bar{O}} \\
\frac{\mathrm{d}}{\mathrm{U}} \\
\frac{\mathrm{O}}{z}\end{array}$ & 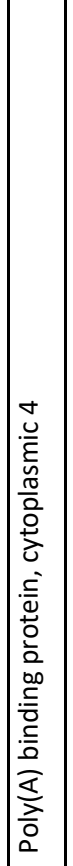 & 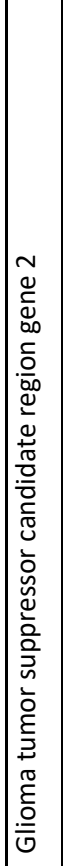 & 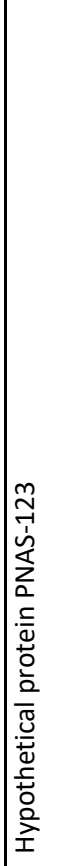 & 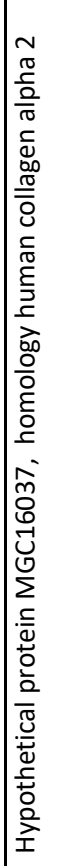 & 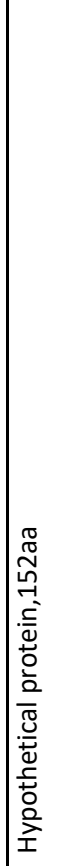 & 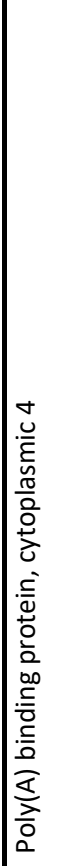 & 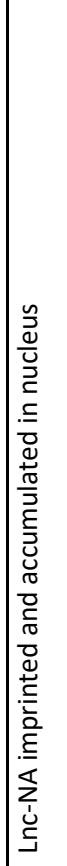 & 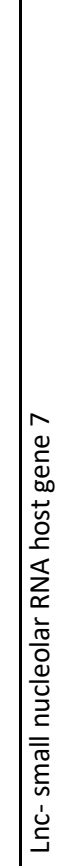 & 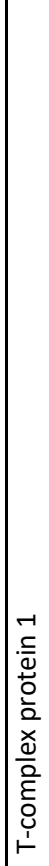 \\
\hline 谷 & mọ & Õ & ơ & $\begin{array}{l}m \\
0 \\
0\end{array}$ & ஜ & @ & \& & ○ & $\begin{array}{l}8 \\
0\end{array}$ & ஜ & $\begin{array}{l}8 \\
0\end{array}$ & $\begin{array}{l}\tilde{O} \\
0 \\
0\end{array}$ & ஜ & 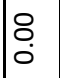 & 8 & 웅 & $\begin{array}{l}\dot{0} \\
0 \\
0\end{array}$ & $\begin{array}{l}\text { Oे } \\
0\end{array}$ & $\begin{array}{l}\text { O } \\
\end{array}$ & Oे \\
\hline 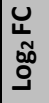 & $\stackrel{\vec{d}}{\stackrel{\sim}{\sim}}$ & $\underset{\substack{\infty \\
\sim}}{\sim}$ & 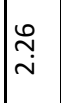 & 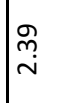 & $\stackrel{m}{\stackrel{m}{\sim}}$ & 胥 & 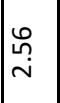 & ְֶ. & $\frac{0}{\stackrel{i}{i}}$ & $\begin{array}{l}9 \\
i \\
i\end{array}$ & $\begin{array}{l}\mathscr{g} \\
\dot{m} \\
\dot{m}\end{array}$ & 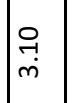 & $\underset{\mathscr{Z}}{\mathcal{Z}}$ & $\begin{array}{l}\qquad 0 \\
0 \\
\text { in }\end{array}$ & 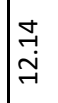 & $\begin{array}{l}\stackrel{P}{+} \\
\stackrel{r}{-}\end{array}$ & $\mid \begin{array}{l}\infty \\
\infty \\
0 \\
0 \\
1\end{array}$ & बू. & 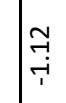 & $\underset{\sim}{\tilde{i}}$ \\
\hline $\begin{array}{l}\stackrel{0}{E} \\
\frac{\pi}{\pi} \\
z\end{array}$ & 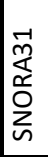 & 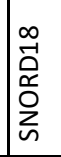 & 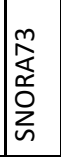 & 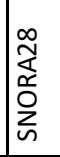 & 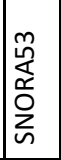 & 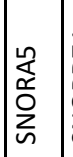 & 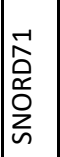 & 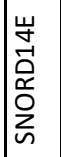 & 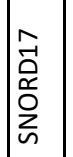 & 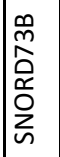 & $\begin{array}{l}\text { O } \\
\text { Qิ } \\
\text { ơ } \\
\text { u } \\
\text { u }\end{array}$ & 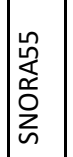 & 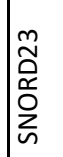 & 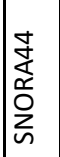 & 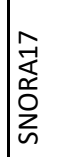 & 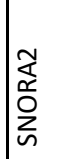 & 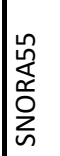 & 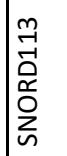 & 辛 & 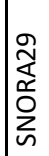 \\
\hline 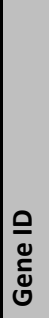 & 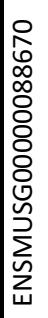 & 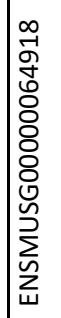 & 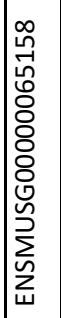 & 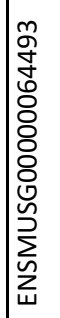 & 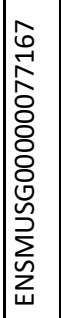 & 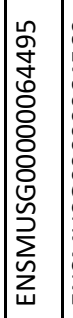 & 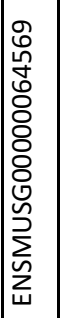 & 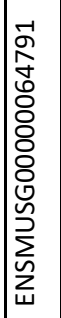 & 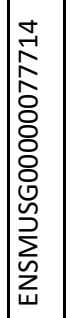 & 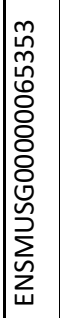 & 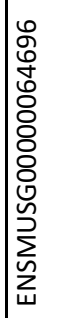 & 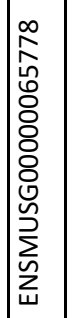 & 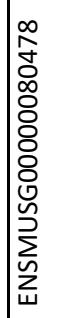 & 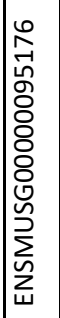 & 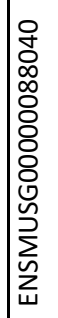 & 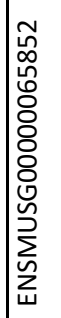 & 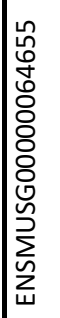 & 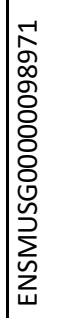 & 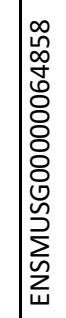 & 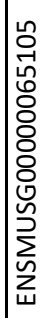 \\
\hline 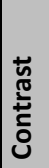 & & & & & & & & & & & & & & & & & & $\begin{array}{l}\stackrel{n}{ } \\
\frac{E}{\pi} \\
\frac{\pi}{\omega}\end{array}$ & 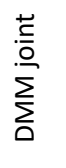 & \\
\hline
\end{tabular}




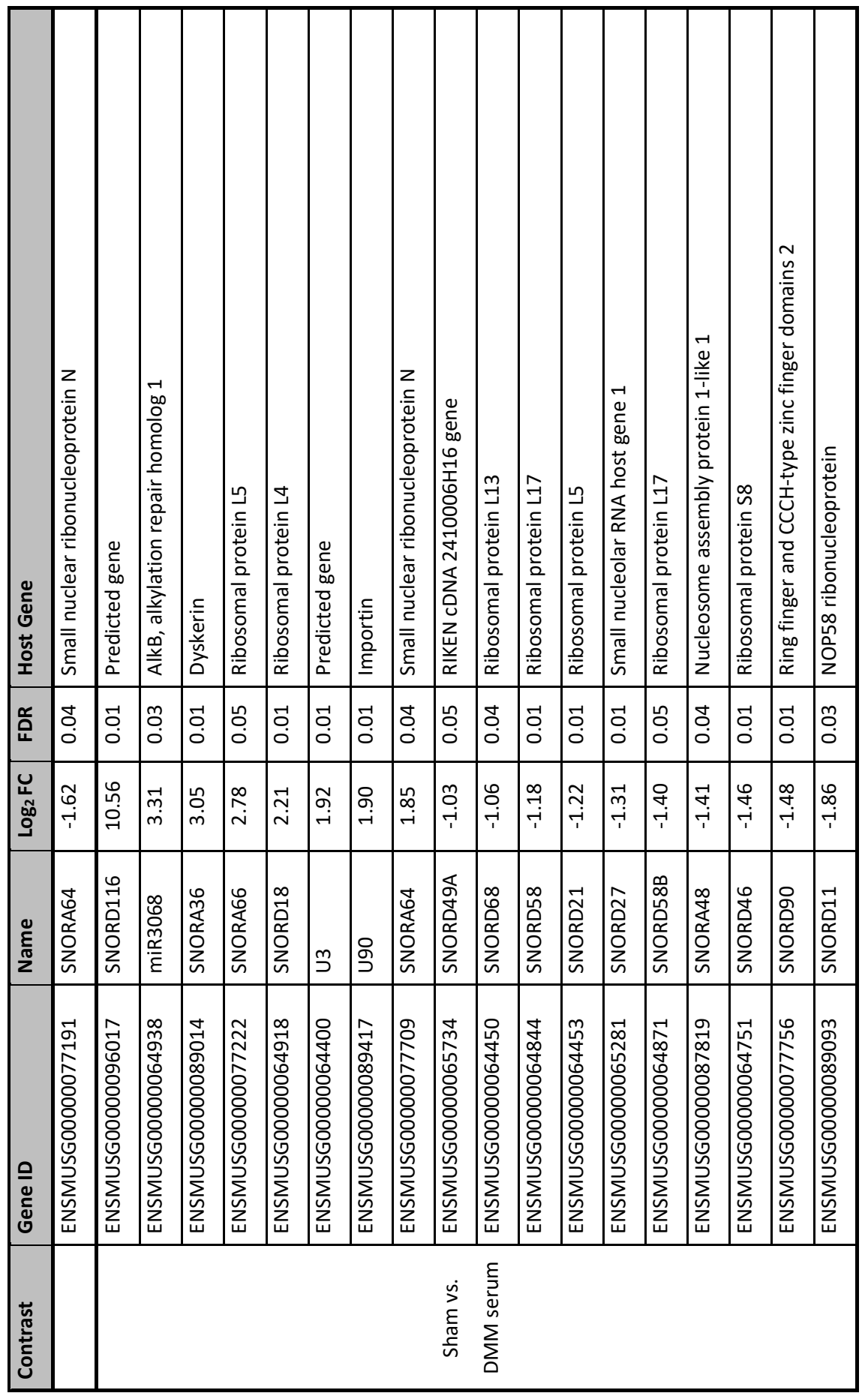




\section{Validation of SnoRNASeq data using qRT-PCR}

Very little is known about the roles of snoRNAs as biomarker for disease or about the functional roles of snoRNAs in mammalian cell biology. We thus could not define functional criteria to select snoRNAs for validation based on prior knowledge. To stay unbiased we thus selected snoRNAs from the RNAseq data that displayed a moderate fold significant expression difference, higher fold significant expression difference, non-significant expression difference and selected box H/ACA (SNORAs) as well as box C/D (SNORDs) snoRNAs for validation. Levels of candidate snoRNAs for further qRT-qPCR analysis were determined using the original RNA from all donors used to perform the SnoRNASeq experiment. There was good concordance between SnoRNASeq and qRT-PCR platforms (Table 2 and Fig. 3). SNORD88 was significantly decreased in young versus old joint (Fig. 3A), while SNORA73 was validated to be increased in young versus old joint (Fig. 3A). In agreement with its absence in the DE group (table 2), SNORA30 was not differentially expressed in young versus old joint (Fig. 3A) and SNORD88 was not differentially expressed in sham versus DMM joint (Fig. 3A). SNORA31, SNORA28, SNORD23 and SNORA73 were confirmed to be significantly increased in young versus old serum (Fig. 3B) and SNORD116, SNORA64 and U3 were significantly increased in sham versus DMM serum (Fig. 3C). SNORD46 was confirmed to be significantly decreased in DMM serum as compared to sham serum (Fig. 3C). 

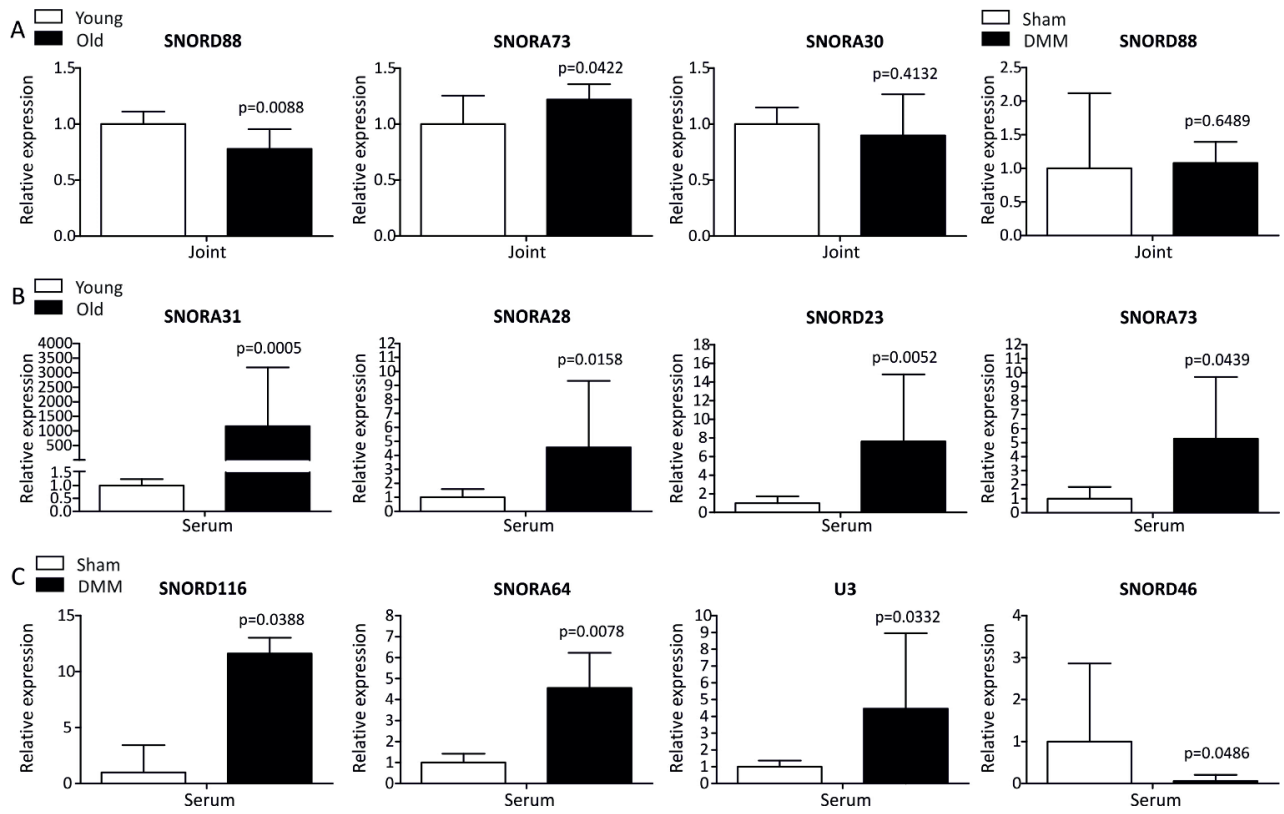

Figure 3. SnoRNA expression from SnoRNASeq was validated with qRT-PCR. A. Gene expression patterns of SNORD88, SNORA73 and SNORA30 were confirmed in young and old joint. SNORD88 expression was verified in sham and DMM joint. B. SNORA31, SNORA28, SNORD23 and SNORA73 were confirmed to be increased in old serum. C. Gene expression patterns of SNORD116, SNORA64, U3 and SNORD46 were validated in sham and DMM serum. Gene expression is depicted as fold induction relative to control (i.e. young or sham). Data represents the mean $+95 \% \mathrm{Cl}$. For statistical evaluation an independent samples t-test was performed using GraphPad Prism 5 (San Diego) on log-transformed data; $p$-values are indicated.

\section{SNORD116 in equine serum in OA}

To confirm increased SNORD116 in OA serum levels in a different species using qRT-PCR, we measured SNORD116 in equine serum samples. Normal equine donors had a Mankin's score $1.25 \pm 0.9$ (mean $\pm 95 \% \mathrm{Cl}$ ) and $\mathrm{OA}$ donors $7.75 \pm$ 7.6. Synovial membrane from normal donors had a synovitis score ${ }^{27}$ of $3.25 \pm 2.3$ and OA donors $3.25 \pm 3.2$. There was a significant increase in serum expression of SNORD116 in OA compared to normal donors $(p=0.0010)$ (Fig. 4). 


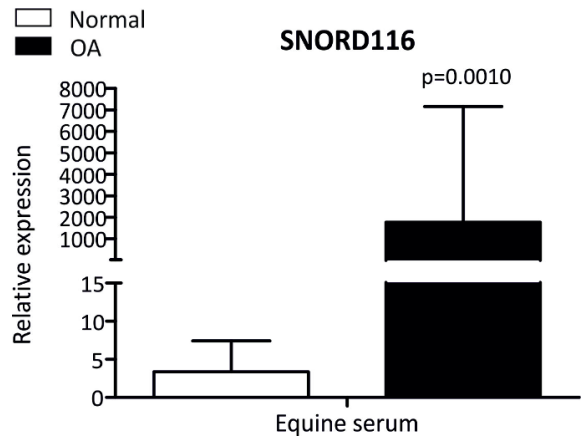

Figure 4. Increased gene expression of SNORD116 in equine OA serum as compared to serum from normal donors. Data represents the mean $+95 \% \mathrm{Cl}$. For statistical evaluation an independent samples t-test was performed using GraphPad Prism 5 (San Diego) on log-transformed data; $p=0.0010$.

\section{Discussion}

SnoRNAs are emerging as important regulators of cell functions, such as alternative splicing $^{28}$, metabolic stress ${ }^{29}$ and development of disease; in cancer ${ }^{6}$, Prader-Willi Syndrome and autism ${ }^{5}$. Through expression profiling of snoRNAs using deep-sequencing we reveal novel molecular features relating to joint ageing and $\mathrm{OA}$.

Whilst gene expression has been evaluated in animal models of $O A$, including the rat anterior cruciate transection ${ }^{30}$ and meniscal tear models ${ }^{31}$, and mouse DMM model ${ }^{32}$ these were primarily interrogating protein-coding genes. Furthermore, apart from the final study these experiments evaluated a single tissue, primarily articular cartilage. In musculoskeletal ageing single tissues have been investigated ${ }^{13,14}$. OA is a process that involves the whole joint as an organ; therefore we undertook our analysis on whole mouse joints, which included cartilage, meniscus, subchondral bone, and joint capsule with synovium.

Mice are considered skeletally mature at around 3-months-old (approximate equivalent of a teenaged human) while a 12 -month-old mouse would signify a 40 to 50 year human ${ }^{33}$. Thus, to investigate the effects of joint age we used 8-month-old equivalent to 25 to 28 year 


\section{Chapter 5}

human (referred to as young) and 24-month-old mice (referred to as old). To study the development of post-traumatic OA we measured OA severity histologically and analysed snoRNAs expression in joints and serum from 24-month-old mice following DMM. Limitations of this study were that we did not additionally undertake the DMM model in young mice. Additionally the 'old' age in young versus old contrast was not the same age as 'old' age in sham versus DMM. OARSI scores that were observed in the sham group (DMM surgery cohort; 24 months old) were lower than the old group (young versus old cohort; 18 months old). At this moment we cannot explain this difference, but different housing and environments of the two cohorts may have influenced this. The DMM model is a post-injury model in which the histological lesions within the affected joint are similar to those observed in human $\mathrm{OA}^{17}$. Mild OA-like pathology was present in the old and sham mice implying that mice at this age are in the early stages of acquiring naturally occurring $\mathrm{OA}$ comparable to studies of human knees at the equivalent age of approximately 40 yearsold $^{34}$. However the effect of the DMM model exacerbated these changes.

Detection of snoRNAs in serum has previously been demonstrated ${ }^{12,35,36}$. SnoRNAs are serum stable, and although normally resident in the nucleolus it is thought in serum they are present as unidentified protein complexes ${ }^{12}$. It is however not clear whether diseaseassociated RNAs detected in the circulation result from local tissue disturbances and cell death, or whether they are actively locally secreted via exosomes or microvesicles or are a systemic response upon local tissue damage ${ }^{8,37,38}$. This may even depend on the specific pathology or specific RNA species. Determining the average read-length distribution of representative joint and serum samples (Figure $2 \mathrm{C}$ ) revealed an unexpected finding. When looking at the read-length distribution the serum reads specifically contain a large peak of RNAs with a length of approximately $30 \mathrm{nt}$. The combined realisation that quite a large portion of the reads could not be mapped back to the used genome database, that a large portion of the piRNA class of non-coding RNAs lacks annotation in the used genome database and that piRNAs are typically $30 \mathrm{nt}$ long, makes it is reasonable to think that this explains the presence of the large $30 \mathrm{nt}$ peak specifically in serum. Only recently the presence of piRNAs in human blood has been described ${ }^{39}$ and this significant difference in the constitution of small RNAs for joint and serum samples is a phenomenon calling for further investigation. 
In the young versus old serum (27 snoRNAs) more snoRNAs were significantly up-regulated compared to the old sham versus old DMM serum (18 snoRNAs) indicating ageing per se had a greater effect on differential snoRNA presence in serum than OA. This could be due to different 'old' ages in the comparisons, but also maybe expected as in ageing serum snoRNAs will be from many tissues whereas in the DMM model we are most likely highlighting primarily OA joint-related snoRNAs. As we were studying the whole joint this may be due to varying expression of snoRNAs in joint tissues, as it is known that there is tissue-specific snoRNAs expression ${ }^{40}$. We are unable to determine the amount each tissue type contributed to overall expression of snoRNAs. Each tissue will vary in its cellularity and hence RNA and snoRNAs content. Thus whilst a novel aspect of this study was that snoRNAs were extracted from the multiple tissues that form the joint, our approach may be less sensitive in detecting snoRNAs that change in a single tissue. However it has the advantage of determining snoRNAs that could be more globally implicated in OA. Despite the potential limitations, we identified a number of potentially interesting snoRNAs for future studies.

SNORA73 was increased in old joint and serum (table 2, Fig. 3) and represents a potential joint 'biological ageing' marker. A reliable measurement of the state of ageing and a prediction of the risk of the onset of morbidity for chronic age-related diseases such as OA would be beneficial. Such a strategy could serve as a measure of 'biological' age and predict an age-related biological response more accurately than chronological age. SNORA64 was increased and SNORD46 was decreased in DMM serum, but both were not DE in young versus old serum, indicating these snoRNAs as possible OA markers. SNORD18 was increased in serum both in ageing and following DMM (table 2) signifying that they are affected in ageing and also OA. It is difficult to speculate how much this snoRNA changes in age-related OA versus age from this study. Histology identified mild increase in OARSI score with age and the level of OA changes in the DMM model was mild. It would be beneficial to investigate this snoRNAs further in tissues and serum in more severe OA.

An interesting finding was an increase in SNORD38 in ageing joint. Zhang et al. ${ }^{12}$ demonstrated a strong association between serum levels of SNORD38 and severe cartilage damage, in anterior cruciate ligament $(A C L)$ injury, enabling distinction between $A C L$ injury 
patients from normal donors. They were unable to determine age effect of SNORD38 in serum from normal donors as it was undetectable. One year post-surgery there was no relationship between donor age and serum SNORD38. Our study demonstrated an increase in SNORD38 in mouse joints (but not serum) with age. The human study did not assess tissue snoRNAs and the serum samples were primarily from middle-aged donors. Our old mice group represents an equivalent older age than that in the human study, which could contribute to this disparity.

The most DE snoRNA in DMM serum was SNORD116. Additionally we demonstrated an increase in serum SNORD116 in horses with MCP OA. We have previously identified SNORD116 as increased in OA compared to normal human cartilage in an array study ${ }^{15}$. A loss of SNORD116 is a significant contribution to the aetiology of the neurodegenerative genetic condition Prader-Willi syndrome (PWS) ${ }^{41}$. This paternally imprinted disorder results in developmental delay and genetic obesity due to hyperphagia ${ }^{42}$. Clinical signs include short stature and low bone mineral density ${ }^{43}$. In a recent mouse transgenic study the loss of PWS critical region (including SNORD116) resulted in reduced bone mineral density (BMD), delayed skeletal development and reduced bone size and osteoblastic suppression ${ }^{44}$. Humans with $\mathrm{OA}$ have an increased $\mathrm{BMD}$ in affected joints ${ }^{45}$. Thus our findings not only elucidate a potential marker of OA but a snoRNA with a potential role in the pathogenesis of OA.

Previously we have identified DE snoRNAs in ageing cartilage ${ }^{13}$ and tendon ${ }^{14}$ and together with results in this study we propose that in musculoskeletal tissues snoRNAs potentially modulate the ageing process as previously described ${ }^{46}$. While determining the transcriptomic signature of ageing equine cartilage ${ }^{13}$ we found the differential expression of a number of snoRNAs associated with ageing. When comparing the differentially expressed snoRNAs between the equine and this mouse study it is important to realize that in our previous equine study we did not analyse serum or the whole joint, but specifically the articular cartilage. Overlapping differential snoRNA expression between studies was identified for SNORD113, SNORA53, SNORA48 and SNORA5. SNORA53 was decreased in old equine cartilage, but increased in old mouse serum. SNORA48 was decreased in old equine cartilage, and decreased in old DMM mouse serum. SNORA5 was increased in old equine 
cartilage and increased in old mouse serum. Possibly the best consistency was found for SNORD113, which was decreased in old mouse joint (and old DMM mouse joint) and equine cartilage. The apparent cross-species conservations of differentially expressed snoRNAs in ageing and OA strengthen our belief that snoRNAs could indeed be used as biomarkers. Further analysis of snoRNA expression profiles and detailed genetic studies will give new insights into novel molecular networks in musculoskeletal ageing and common mechanisms in ageing and age-related diseases such as OA.

In mammalians the majority of snoRNAs are encoded within the introns of protein coding or non-coding genes; host-genes ${ }^{47}$. There is evidence that genes which host snoRNAs might contribute to the aetiology of cancer through regulation of cell homeostasis and cancer biology $\left(\right.$ reviewed $\left.^{6}\right)$. Potentially both the host-gene and the snoRNAs encoded within them may be important in different situations. For example growth-arrest-specific-5 (GAS-5) (hosts ten C/D box snoRNAs ${ }^{48}$ ), a non-coding RNA which accumulates in growth arrested cells, regulates cell death and proliferation by acting as a decoy hormone response element for glucocorticoid receptors thereby inhibiting gene upregulation by activated glucocorticoid receptors ${ }^{49}$. Interesting snoRNA host-genes were identified in this study including transforming growth factor $\beta$ regulated gene 4 , sorting nexin 5 and collagen type 1 (with roles in joint homeostasis). Therefore, an alteration of snoRNA expression may result from changes in transcriptional activity of the host-gene related to joint homeostasis or disease.

\section{Conclusion}

Our results implicate specific changes in snoRNA abundance in joint ageing (SNORD88 and SNORD38 were respectively decreased and increased) and OA suggesting the potential use of snoRNAs such as SNORA73 and SNORD23 as a novel biomarker for joint ageing, SNORA64, SNORD46 and SNORD116 for OA, SNORD18 for ageing and OA. 


\section{Acknowledgements}

We thank George Bou-Gharios, Alan Carter, James Anderson and Yalda Ashraf Kharaz for assistance with histological scoring and Andy Cremers for technical assistance with the snoRNA qRT-PCR. The authors wish to thank the Wellcome Trust for MJP's Wellcome Trust Clinical Intermediate Fellowship (grant 107471/Z/15/Z) and the Medical Research Council (MRC) and Arthritis Research UK as part of the MRC-Arthritis Research UK Centre for Integrated research into Musculoskeletal Ageing (CIMA) (MJP.PC), NWO-DFG (grant \#DN82304; NL; TJMW) and the Dutch Arthritis Foundation (grant \#LLP14; NL; TJMW) for funding; Chris Riggs, Hong Kong Jockey Club for equine serum sample provision.

\section{Authors Contributions}

MJP, MMFS; conception and design, collection and assembly of data, data analysis and interpretation, manuscript writing, final approval of the manuscript. YF; assembly of data, data analysis, manuscript writing, final approval of the manuscript. PM; provision of study material, assembly of data, manuscript writing and final approval of the manuscript. PDC, TJMW, DY; conception and design, manuscript writing, final approval of the manuscript.

\section{Role of the funding source}

Funding sources are (MJP,PC) Wellcome Trust Clinical Intermediate Fellowship (grant 107471/Z/15/Z) and the Medical Research Council (MRC) and Arthritis Research UK as part of the MRC-Arthritis Research UK Centre for Integrated research into Musculoskeletal Ageing (CIMA) (MJP.PC), NWO-DFG (grant \#DN82-304; NL; TJMW) and the Dutch Arthritis Foundation (grant \#LLP14; NL; TJMW). 


\section{Competing Interests Statement}

We declare no competing interests.

\section{Supplementary files}

Supplementary file 1. Raw reads. Summaries of raw and trimmed reads.

\begin{tabular}{|c|c|c|c|c|c|}
\hline \#Sample & $\mathrm{BC}$ & \# of Raw Read & \# of Trimmed Read ( $\%$ of raw) & \# of R1/R2 & \# of R0 ( $\%$ of trimmed reads) \\
\hline Sample_24-DMM1_joint & GATCAG & 23.782 .170 & $22,715,170(95.51)$ & 11.042 .905 & $629,360(2.77)$ \\
\hline Sample_25-DMM2_joint & TAGCTT & 21.854 .482 & $20,833,718(95.33)$ & 10.104 .436 & $624,846(3.00)$ \\
\hline Sample_26-DMM3_joint & GGCTAC & 17.865 .238 & $16,897,771(94.58)$ & 8.149 .593 & $598,585(3.54)$ \\
\hline Sample_27-DMM4_joint & GATCAG & 22.398 .708 & $21,080,467$ (94.11) & 10.171 .809 & $736,849(3.50)$ \\
\hline Sample_28-DMM5_joint & TAGCTT & 28.684 .964 & $27,200,017(94.82)$ & 13.203 .107 & $793,803(2.92)$ \\
\hline Sample_29-DMM6_joint & GGCTAC & 22.312 .038 & $20,883,289(93.60)$ & 10.064 .250 & $754,789(3.61)$ \\
\hline Sample_30-Sham1_joint & ACTTGA & 21.967 .478 & $20,340,819(92.60)$ & 9.726 .059 & $888,701(4.37)$ \\
\hline Sample_31-Sham2_joint & GATCAG & 24.467 .412 & $22,913,228$ (93.65) & 11.123 .732 & $665,764(2.91)$ \\
\hline Sample_32-Sham3_joint & TAGCTT & 24.033 .750 & $22,542,043(93.79)$ & 10.847 .687 & $846,669(3.76)$ \\
\hline Sample_33-DMM1_serum & CTTGTA & 21.365 .546 & $20,222,190(94.65)$ & 9.860 .582 & $501,026(2.48)$ \\
\hline Sample_34-DMM2_serum & AGTCAA & 22.293.396 & $21,429,767(96.13)$ & 10.511 .204 & $407,359(1.90)$ \\
\hline Sample_35-DMM3_serum & AGTTCC & 22.539 .880 & $21,591,483(95.79)$ & 10.596 .322 & $398,839(1.85)$ \\
\hline Sample_36-DMM4_serum & CTTGTA & 23.275 .974 & $21,841,826(93.84)$ & 10.710 .036 & $421,754(1.93)$ \\
\hline Sample_37-DMM5_serum & AGTCAA & 25.526 .080 & $23,953,737(93.84)$ & 11.741 .359 & $471,019(1.97)$ \\
\hline Sample_38-DMM6_serum & AGTTCC & 21.568 .244 & $20,364,282(94.42)$ & 9.985 .206 & $393,870(1.93)$ \\
\hline Sample_39-Sham1_serum & GGCTAC & 21.641 .998 & $20,234,385(93.50)$ & 9.938 .472 & $357,441(1.77)$ \\
\hline Sample_40-Sham2_serum & CTTGTA & 20.457 .350 & $19,252,436(94.11)$ & 9.445 .278 & $361,880(1.88)$ \\
\hline Sample_41-Sham3_serum & AGTCAA & 22.631 .824 & $21,646,393(95.65)$ & 10.657 .755 & $330,883(1.53)$ \\
\hline Sample_7-94_old_joint & TTAGGC & 25.449 .962 & $24,069,261(94.57)$ & 11.674 .995 & $719,271(2.99)$ \\
\hline Sample_8-95_old_joint & TGACCA & 26.136 .496 & $24,981,407(95.58)$ & 12.182 .606 & $616,195(2.47)$ \\
\hline Sample_9-96_old_joint & ATCACG & 24.812 .536 & $23,680,611(95.44)$ & 11.594.217 & $492,177(2.08)$ \\
\hline Sample_10-97_old_joint & CGATGT & 25.469 .668 & $23,967,220(94.10)$ & 11.618 .236 & $730,748(3.05)$ \\
\hline Sample_1-106_old_joint & ATCACG & 24.709 .064 & $23,797,278$ (96.31) & 11.612 .999 & $571,280(2.40)$ \\
\hline Sample_11-98_old_joint & TTAGGC & 26.961 .846 & $24,359,756(90.35)$ & 11.312 .655 & $1,734,446(7.12)$ \\
\hline Sample_12-99_old_joint & TGACCA & 26.503 .992 & $25,035,419(94.46)$ & 12.144 .470 & $746,479(2.98)$ \\
\hline Sample_19-94_old_serum & CAGATC & 24.453 .146 & $22,591,704$ (92.39) & 10.895 .811 & $800,082(3.54)$ \\
\hline Sample_20-95_old_serum & ACTTGA & 25.385 .730 & $23,483,541$ (92.51) & 11.395 .156 & $693,229(2.95)$ \\
\hline Sample_2-107_old_joint & CGATGT & 19.992 .488 & $19,261,359(96.34)$ & 9.402 .499 & $456,361(2.37)$ \\
\hline Sample_21-96_old_serum & ACAGTG & 21.474 .502 & $19,955,318(92.93)$ & 9.725 .699 & $503,920(2.53)$ \\
\hline Sample_22-97_old_serum & GCCAAT & 25.188.282 & $23,515,257(93.36)$ & 11.508 .820 & $497,617(2.12)$ \\
\hline Sample_23-98_old_serum & CAGATC & 25.737 .064 & $23,941,043(93.02)$ & 11.731 .780 & $477,483(1.99)$ \\
\hline Sample_3-108_old_joint & TTAGGC & 26.680 .444 & $25,712,825(96.37)$ & 12.567 .907 & $577,011(2.24)$ \\
\hline Sample_4-109_old_joint & TGACCA & 23.528 .796 & $22,718,211(96.55)$ & 11.119 .890 & $478,431(2.11)$ \\
\hline Sample_5-110_old_joint & ATCACG & 21.863 .890 & $20,853,075(95.38)$ & 10.173 .805 & $505,465(2.42)$ \\
\hline Sample_6-111_old_joint & CGATGT & 26.489 .526 & $25,143,207$ (94.92) & 12.244 .533 & $654,141(2.60)$ \\
\hline Sample_13-106_old_serum & ACAGTG & 22.167 .284 & $21,348,062(96.30)$ & 10.482 .680 & $382,702(1.79)$ \\
\hline Sample_14-107_old_serum & GCCAAT & 24.214 .362 & $22,603,964$ (93.35) & 11.078 .395 & $447,174(1.98)$ \\
\hline Sample_15-108_old_serum & CAGATC & 22.697 .452 & $21,302,562(93.85)$ & 10.441 .322 & $419,918(1.97)$ \\
\hline Sample_16-109_old_serum & ACTTGA & 21.958 .590 & $20,571,219(93.68)$ & 10.079 .860 & $411,499(2.00)$ \\
\hline Sample_17-110_old_serum & ACAGTG & 21.976 .864 & $20,608,191(93.77)$ & 10.108 .567 & $391,057(1.90)$ \\
\hline Sample_18-111_old_serum & GCCAAT & 22.578 .298 & $21,415,267(94.85)$ & 10.493 .200 & $428,867(2.00)$ \\
\hline
\end{tabular}


Supplementary file 2. Mapped reads. Summaries of sequencing alignment to mouse snoRNAs.

Samples \#ReadsToAlign NumberAlignedReads \%ofAlignment \#snoRNAhit \%snoRNAhit

\begin{tabular}{|c|c|c|c|c|c|}
\hline Sample_1-106_old_joint & 11.612 .999 & 2.369 .475 & 20,40 & 738 & 47,46 \\
\hline Sample_2-107_old_joint & 9.402 .499 & 2.090 .100 & 22,23 & 648 & 41,67 \\
\hline Sample_3-108_old_joint & 12.567 .907 & 3.669 .200 & 29,20 & 672 & 43,22 \\
\hline Sample_4-109_old_joint & 11.119 .890 & 2.567 .057 & 23,09 & 729 & 46,88 \\
\hline Sample_5-110_old_joint & 10.173 .805 & 2.029 .817 & 19,95 & 755 & 48,55 \\
\hline Sample_6-111_old_joint & 12.244 .533 & 2.461 .834 & 20,11 & 808 & 51,96 \\
\hline Sample_7-94_old_joint & 11.674 .995 & 2.029 .233 & 17,38 & 779 & 50,10 \\
\hline Sample_8-95_old_joint & 12.182 .606 & 2.273 .304 & 18,66 & 807 & 51,90 \\
\hline Sample_9-96_old_joint & 11.594 .217 & 3.148 .773 & 27,16 & 753 & 48,42 \\
\hline Sample_10-97_old_joint & 11618236 & 2649996 & 22,8089 & 821 & 52,7974 \\
\hline Sample_11-98_old_joint & 11.312 .655 & 2.389 .431 & 21,12 & 793 & 51,00 \\
\hline Sample_12-99_old_joint & 12.144 .470 & 1.887 .034 & 15,54 & 827 & 53,18 \\
\hline Sample_13-106_old_serum & 10.482 .680 & 3.441 & 0,03 & 273 & 17,56 \\
\hline Sample_14-107_old_serum & 11.078 .395 & 5.350 & 0,05 & 295 & 18,97 \\
\hline Sample_15-108_old_serum & 10.441 .322 & 5.785 & 0,06 & 307 & 19,74 \\
\hline Sample_16-109_old_serum & 10.079 .860 & 5.267 & 0,05 & 299 & 19,23 \\
\hline Sample_17-110_old_serum & 10.108 .567 & 6.756 & 0,07 & 295 & 18,97 \\
\hline Sample_18-111_old_serum & 10.493 .200 & 4.422 & 0,04 & 332 & 21,35 \\
\hline Sample_19-94_old_serum & 10.895 .811 & 97.983 & 0,90 & 403 & 25,92 \\
\hline Sample_20-95_old_serum & 11395156 & 50234 & 0,440836 & 334 & 21,4791 \\
\hline Sample_21-96_old_serum & 9.725 .699 & 3.618 & 0,04 & 296 & 19,04 \\
\hline Sample_22-97_old_serum & 11.508 .820 & 19.327 & 0,17 & 337 & 21,67 \\
\hline Sample_23-98_old_serum & 11.731 .780 & 6.316 & 0,05 & 368 & 23,67 \\
\hline Sample_24-DMM1_joint & 11.042 .905 & 1.410 .182 & 12,77 & 740 & 47,59 \\
\hline Sample_25-DMM2_joint & 10.104 .436 & 1.674 .606 & 16,57 & 701 & 45,08 \\
\hline Sample_26-DMM3_joint & 8.149 .593 & 1.414 .854 & 17,36 & 648 & 41,67 \\
\hline Sample_27-DMM4_joint & 10.171 .809 & 1.865 .187 & 18,34 & 775 & 49,84 \\
\hline Sample_28-DMM5_joint & 13.203.107 & 1.587 .933 & 12,03 & 851 & 54,73 \\
\hline Sample_29-DMM6_joint & 10.064 .250 & 1.199 .565 & 11,92 & 724 & 46,56 \\
\hline Sample_30-Sham1_joint & 9.726 .059 & 1.434 .498 & 14,75 & 749 & 48,17 \\
\hline Sample_31-Sham2_joint & 11.123.732 & 1.955 .315 & 17,58 & 760 & 48,87 \\
\hline Sample_32-Sham3_joint & 10.847 .687 & 1.335 .491 & 12,31 & 814 & 52,35 \\
\hline Sample_33-DMM1_serum & 9.860 .582 & 12.734 & 0,13 & 326 & 20,96 \\
\hline Sample_34-DMM2_serum & 10.511 .204 & 3.325 & 0,03 & 257 & 16,53 \\
\hline Sample_35-DMM3_serum & 10.596 .322 & 3.032 & 0,03 & 249 & 16,01 \\
\hline Sample_36-DMM4_serum & 10.710 .036 & 8.664 & 0,08 & 296 & 19,04 \\
\hline Sample_37-DMM5_serum & 11.741 .359 & 11.980 & 0,10 & 366 & 23,54 \\
\hline Sample_38-DMM6_serum & 9.985 .206 & 10.162 & 0,10 & 353 & 22,70 \\
\hline Sample_39-Sham1_serum & 9.938 .472 & 6.476 & 0,07 & 303 & 19,49 \\
\hline Sample_40-Sham2_serum & 9.445 .278 & 10.939 & 0,12 & 325 & 20,90 \\
\hline Sample_41-Sham3_serum & 10.657 .755 & 58.724 & 0,55 & 364 & 23,41 \\
\hline
\end{tabular}




\section{References}

1 Woolf, A. D. \& Pfleger, B. Burden of major musculoskeletal conditions. Bull World Health Organ 81, 646-656 (2003).

2 Hunter, D. J., Schofield, D. \& Callander, E. The individual and socioeconomic impact of osteoarthritis. Nat Rev Rheumatol 10, 437-441, doi:10.1038/nrrheum.2014.44 (2014).

3 Li, Y., Wei, X., Zhou, J. \& Wei, L. The age-related changes in cartilage and osteoarthritis. Biomed Res Int 2013, 916530, doi:10.1155/2013/916530 (2013).

Ishijima, M., Kaneko, H. \& Kaneko, K. The evolving role of biomarkers for osteoarthritis. Ther Adv Musculoskelet Dis 6, 144-153, doi:10.1177/1759720X14541175 (2014).

5 Stepanov, G. A. et al. Regulatory role of small nucleolar RNAs in human diseases. Biomed Res Int 2015, 206849, doi:10.1155/2015/206849 (2015).

6 Williams, G. T. \& Farzaneh, F. Are snoRNAs and snoRNA host genes new players in cancer? Nat Rev Cancer 12, 84-88, doi:10.1038/nrc3195 (2012).

7 Tada, M. et al. [IPMN and pancreatic cyst as high risk of pancreatic cancer]. Nihon Shokakibyo Gakkai Zasshi 112, 1474-1478, doi:10.11405/nisshoshi.112.1474 (2015).

8 Kishikawa, T. et al. Circulating RNAs as new biomarkers for detecting pancreatic cancer. World J Gastroenterol 21, 8527-8540, doi:10.3748/wjg.v21.i28.8527 (2015).

9 Cortez, M. A., Welsh, J. W. \& Calin, G. A. Circulating microRNAs as noninvasive biomarkers in breast cancer. Recent Results Cancer Res 195, 151-161, doi:10.1007/978-3-642-28160-0_13 (2012).

10 Galimberti, D. et al. Circulating miRNAs as potential biomarkers in Alzheimer's disease. J Alzheimers Dis 42, 1261-1267, doi:10.3233/JAD-140756 (2014).

11 Mi, S., Zhang, J., Zhang, W. \& Huang, R. S. Circulating microRNAs as biomarkers for inflammatory diseases. Microrna 2, 63-71, doi:10.2174/2211536611302010007 (2013).

12 Zhang, L. et al. Serum non-coding RNAs as biomarkers for osteoarthritis progression after ACL injury. Osteoarthritis Cartilage 20, 1631-1637, doi:10.1016/j.joca.2012.08.016 (2012).

13 Peffers, M., Liu, X. \& Clegg, P. Transcriptomic signatures in cartilage ageing. Arthritis Res Ther 15, R98, doi:10.1186/ar4278 (2013).

14 Peffers, M. J. et al. Transcriptome analysis of ageing in uninjured human Achilles tendon. Arthritis Res Ther 17, 33, doi:10.1186/s13075-015-0544-2 (2015).

Peffers, M. J. et al. BIG tasks for small RNAs; a new class of rnas in the pathgenesis of osteoarthritis. Osteoarthritis and Cartilage 24, S372 (2016).

16 Duran, A. L. et al. Shared Ageing Research Models (ShARM): a new facility to support ageing research. Biogerontology 14, 789-794, doi:10.1007/s10522-013-9457-0 (2013). 
17 Glasson, S. S., Blanchet, T. J. \& Morris, E. A. The surgical destabilization of the medial meniscus (DMM) model of osteoarthritis in the 129/SvEv mouse. Osteoarthritis Cartilage 15, 1061-1069, doi:10.1016/j.joca.2007.03.006 (2007).

18 Glasson, S. S., Chambers, M. G., Van Den Berg, W. B. \& Little, C. B. The OARSI histopathology initiative - recommendations for histological assessments of osteoarthritis in the mouse. Osteoarthritis Cartilage 18 Suppl 3, S17-23, doi:10.1016/j.joca.2010.05.025 (2010).

19 http://support.illumina.com/content/dam/illuminasupport/documents/myillumina/a557afc4-bf0e-4dad-9e599c740dd1e751/casava userguide 15011196d.pdf. CASAVA v1.8.2 User Guide, 2011).

20 Martin, M. Cutadapt removes adapter sequences from high-throughput sequencing reads. EMBnet. Journal 17, 10-12 (2011).

21 Langmead, B. \& Salzberg, S. L. Fast gapped-read alignment with Bowtie 2. Nat Methods 9, 357-359, doi:10.1038/nmeth.1923 (2012).

22 Robinson, M. D., McCarthy, D. J. \& Smyth, G. K. edgeR: a Bioconductor package for differential expression analysis of digital gene expression data. Bioinformatics 26, 139-140, doi:10.1093/bioinformatics/btp616 (2010).

23 Wilks, S. S. The Large-Sample Distribution of the Likelihood Ratio for Testing Composite Hypotheses. 9, 60-62 (1938).

24 Seungil, R., Chanjae, P., Jingling, J., Sanders, K. M. \& Wei, Y. A PCR-based method for detection and quantification of small RNAs. Elsevier Biochemical and Biophysical Research Communications 351, 756-763 (2006).

25 McCoy, A. M. Animal Models of Osteoarthritis: Comparisons and Key Considerations. Vet Pathol 52, 803-818, doi:10.1177/0300985815588611 (2015).

26 Moskowitz, R. W. Osteoarthritis cartilage histopathology: grading and staging. Osteoarthritis Cartilage 14, 1-2, doi:10.1016/j.joca.2005.08.015 (2006).

27 Krenn, V. et al. Synovitis score: discrimination between chronic low-grade and highgrade synovitis. Histopathology 49, 358-364, doi:10.1111/j.1365-2559.2006.02508.x (2006).

28 Kishore, S. et al. The snoRNA MBII-52 (SNORD 115) is processed into smaller RNAs and regulates alternative splicing. Hum Mol Genet 19, 1153-1164, doi:10.1093/hmg/ddp585 (2010).

29 Michel, C. I. et al. Small nucleolar RNAs U32a, U33, and U35a are critical mediators of metabolic stress. Cell Metab 14, 33-44, doi:10.1016/j.cmet.2011.04.009 (2011).

30 Appleton, C. T., Pitelka, V., Henry, J. \& Beier, F. Global analyses of gene expression in early experimental osteoarthritis. Arthritis Rheum 56, 1854-1868, doi:10.1002/art.22711 (2007).

31 Wei, T. et al. Analysis of early changes in the articular cartilage transcriptisome in the rat meniscal tear model of osteoarthritis: pathway comparisons with the rat anterior cruciate transection model and with human osteoarthritic cartilage. Osteoarthritis Cartilage 18, 992-1000, doi:10.1016/j.joca.2010.04.012 (2010). 
Loeser, R. F. et al. Microarray analysis reveals age-related differences in gene expression during the development of osteoarthritis in mice. Arthritis Rheum 64, 705-717, doi:10.1002/art.33388 (2012).

33 Turnbull, I. R. et al. Effects of age on mortality and antibiotic efficacy in cecal ligation and puncture. Shock 19, 310-313 (2003).

34 Muehleman, C., Margulis, A., Bae, W. C. \& Masuda, K. Relationship between knee and ankle degeneration in a population of organ donors. BMC Med 2010, 48.

35 Okugawa, Y. et al. Clinical significance of SNORA42 as an oncogene and a prognostic biomarker in colorectal cancer. Gut, doi:10.1136/gutjnl-2015-309359 (2015).

Liao, J. et al. Small nucleolar RNA signatures as biomarkers for non-small-cell lung cancer. Mol Cancer 9, 198, doi:10.1186/1476-4598-9-198 (2010).

37 Hunter, M. P. et al. Detection of microRNA expression in human peripheral blood microvesicles. PLoS One 3, e3694, doi:10.1371/journal.pone.0003694 (2008).

Enderle, D. et al. Characterization of RNA from Exosomes and Other Extracellular Vesicles Isolated by a Novel Spin Column-Based Method. PLoS One 10, e0136133, doi:10.1371/journal.pone.0136133 (2015).

39 Yang, X. et al. Detection of stably expressed piRNAs in human blood. Int J Clin Exp Med 8, 13353-13358 (2015).

40 Huttenhofer, A. et al. RNomics: an experimental approach that identifies 201 candidates for novel, small, non-messenger RNAs in mouse. EMBO J 20, 2943-2953, doi:10.1093/emboj/20.11.2943 (2001).

41 Duker, A. L. et al. Paternally inherited microdeletion at $15 q 11.2$ confirms a significant role for the SNORD116 C/D box snoRNA cluster in Prader-Willi syndrome. Eur J Hum Genet 18, 1196-1201, doi:10.1038/ejhg.2010.102 (2010).

42 Holm, V. A. et al. Prader-Willi syndrome: consensus diagnostic criteria. Pediatrics 91, 398-402 (1993).

43 Butler, M. G. et al. Decreased bone mineral density in Prader-Willi syndrome: comparison with obese subjects. Am J Med Genet 103, 216-222 (2001).

44 Khor, E. C. et al. Prader-Willi Critical Region, a Non-Translated, Imprinted Central Regulator of Bone Mass: Possible Role in Skeletal Abnormalities in Prader-Willi Syndrome. PLoS One 11, e0148155, doi:10.1371/journal.pone.0148155 (2016).

45 Karlsson, M. K., Magnusson, H., Coster, M., Karlsson, C. \& Rosengren, B. E. Patients with knee osteoarthritis have a phenotype with higher bone mass, higher fat mass, and lower lean body mass. Clin Orthop Relat Res 473, 258-264, doi:10.1007/s11999014-3973-3 (2015).

46 Kato, M., Chen, X., Inukai, S., Zhao, H. \& Slack, F. J. Age-associated changes in expression of small, noncoding RNAs, including microRNAs, in C. elegans. RNA 17, 1804-1820, doi:10.1261/rna.2714411 (2011).

47 Bachellerie, J. P., Cavaille, J. \& Huttenhofer, A. The expanding snoRNA world. Biochimie 84, 775-790 (2002). 
Chapter 5

48 Kino, T., Hurt, D. E., Ichijo, T., Nader, N. \& Chrousos, G. P. Noncoding RNA gas5 is a growth arrest- and starvation-associated repressor of the glucocorticoid receptor. Sci Signal 3, ra8, doi:10.1126/scisignal.2000568 (2010).

49 Smith, C. M. \& Steitz, J. A. Classification of gas5 as a multi-small-nucleolar-RNA (snoRNA) host gene and a member of the 5'-terminal oligopyrimidine gene family reveals common features of snoRNA host genes. Mol Cell Biol 18, 6897-6909 (1998). 
Serum snoRNAs as biomarkers for joint ageing and post traumatic osteoarthritis 



\section{Chapter 6}

General discussion 
Chapter 6 


\section{Targets of snoRNAs and their effect on cell function, chondrogenic development and cartilage pathology}

The cellular processes in which snoRNAs participate are fundamental processes needed for proper cell function, yet their roles in cell differentiation, homeostasis and disease in general have been poorly investigated. Moreover, in the context of chondrogenic differentiation, cartilage homeostasis and disease, snoRNAs have not been investigated so far. In this thesis we therefore investigated the involvement of snoRNAs in several chondrocytic / cartilage facets and linked them to development and disease. As becomes clear from the previous chapters, snoRNAs exert their effects upon a broad array of functionally different targets, which consequently influences cell function in different ways. Canonical snoRNAs such as the box C/D and box H/ACA snoRNAs, function in the posttranscriptional modification of target RNAs. Non-canonical snoRNAs, such as RMRP snoRNA ${ }^{1,2}$ or U3 snoRNA ${ }^{3}$, perform endoribonucleolytic cleavage of their targets ${ }^{1-3}$. In the following paragraphs we will discuss different targets of snoRNAs and their effect on cell function, chondrogenic development and cartilage pathologies such as Cartilage-Hair Hypoplasia $(\mathrm{CHH})$ and osteoarthritis $(\mathrm{OA})$. With regard to cartilage pathologies it will also be discussed how snoRNAs could potentially be utilized as biomarkers for osteoarthritis (Figure 1). In this general discussion we will specifically focus on mRNA and rRNA targets of snoRNAs. As briefly noted in the general introduction, snoRNA can also chemically modify snRNAs ${ }^{4-6}$, however this aspect will not be discussed here. 


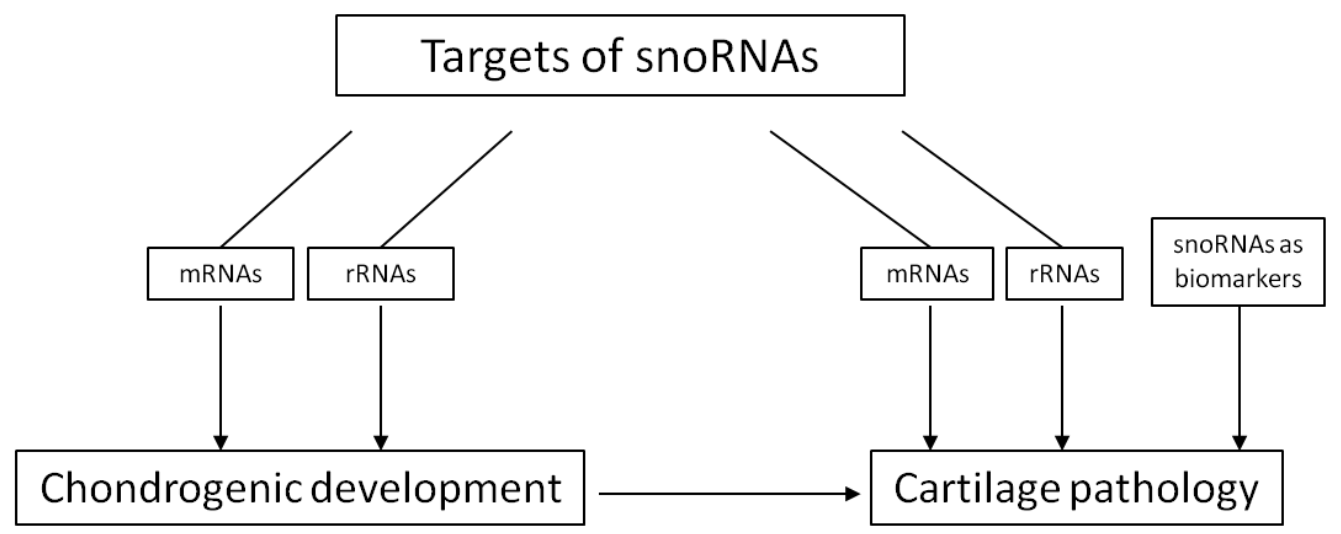

Figure 1. Overview of discussion subjects addressed in this thesis. snoRNA targets and their effect on cell function in chondrogenic development and cartilage pathology. The role of snoRNAs as biomarkers for cartilage pathology, and osteoarthritis in particular, will also be discussed.

\section{1 mRNA targets of RMRP snoRNA and their roles in chondrogenic development and disease}

In chapter 2 we have seen that the RNase MRP complex, a small nucleolar ribonucleoprotein particle consisting of the RMRP snoRNA and several protein subunits ${ }^{7}$, fulfills many functions. The complex has been reported to be involved in mitochondrial DNA replication $^{8}$, cleaves pre-rRNA in the internal transcribed spacer 1 (ITS1) $)^{1,2}$, plays a role in cell cycle regulation by cleaving cyclin B2 (Clb2) $\mathrm{mRNA}^{9}$, and is able to cleave viperin $\mathrm{mRNA}^{10}$. Cyclin B2 cleavage and viperin mRNA cleavage are clear examples of how a noncanonical snoRNA, in this case RMRP snoRNA, performs endoribonucleolytic cleavage of mRNA targets and in chapter 3, we explored how this influences chondrogenic differentiation. In $\mathrm{CHH}, R M R P$ is mutated and its expression levels are reduced, resulting in reduced substrate cleavage and consequently an increased expression of viperin mRNA ${ }^{10,11}$. Our results indicate that the antiviral protein viperin controls chondrodrogenic differentiation by influencing secretion of soluble proteins and we identified a viperinCXCL10-TGF- $\beta /$ SMAD2/3 regulatory axis controlling chondrogenic differentiation, which is deregulated in chondrocytic $\mathrm{CHH}$ cells (chapter 3$)^{12}$. This clearly demonstrates that 
downstream RNase MRP mRNA targets have important roles in cellular function and development. However at this point it remains largely unexplored how RNase MRPmediated cleavage of mRNAs is controlled.

\subsubsection{Transcriptional control of RMRP snoRNA by chondrogenic mediators}

Our observations that expression of RNase MRP components is modulated in differentiating cells prompted us to investigate whether RMRP snoRNA expression levels can be controlled at the transcription level. In one of our studies (chapter 2 ) we found that expression of RMRP snoRNA indeed responds to different extracellular morphogens ${ }^{11}$. PTHrP, which keeps chondrocytes in a proliferative state ${ }^{13,14}$, decreased the Rmrp promoter activity. In addition, bFGF/FGF2, a negative regulator of chondrocyte hypertrophic maturation ${ }^{15}$, reduced $R m r p$ promoter activity. It remains to be determined whether reduced Rmrp promoter activity and RMRP snoRNA expression by PTHrP and bFGF is an indirect result of these morphogens altering the general chondrocyte phenotype (for example by modulating WNT / BMP signaling) or that PTHrP and bFGF signaling are able to directly control Rmrp expression by inhibition of Rmrp promoter activity. TGF- $\beta 3$, described to be able to induce matrix mineralization, but which was also shown to have an important role in chondrogenic differenctiation ${ }^{16-18}$, increased Rmrp promoter activity. In agreement with the TGF- $\beta 3$ induced increase of Rmrp promoter activity, exposure to (5Z)-7-Oxozeaenol, an inhibitor of TGF- $\beta$ activated kinase- $1^{19}$, decreased Rmrp promoter activity. Likewise, exposure to the pro-hypertrophic BMP-2 $2^{20}$ increased Rmrp promoter activity, whereas dorsomorphin, an inhibitor of BMP-mediated SMAD1/5/8 phosphorylation ${ }^{21}$, decreased $R m r p$ promoter activity. Finally, WNT-3A and WNT-5A, two prominent ligands of the Wnt signaling pathway and crucial in chondrocyte differentiation ${ }^{22}$, increased $R m r p$ promoter activity. RMRP RNA expression is driven by RNA polymerase $1 \mathrm{II}^{23}$. However, transcriptional regulation by RNA polymerase III is poorly understood. To regulate promoter activity and to be able to respond to an altering demand for RMRP snoRNA during for example chondrocyte hypertrophy, it is expected that additional transcription regulatory elements are required. In an example from the cancer research field, it has been shown that $R M R P$ transcription in colon cancer is driven by WNT-3A, via activation of $\beta$-catenin and YAP proteins ${ }^{24}$. The responsiveness to 


\section{Chapter 6}

chondrocyte morphogens described in this thesis corroborates the theory that the 1500 base pair sequence upstream of the RMRP transcription start site is under transcriptional control of chondrogenic cues that are associated with chondrocyte differentiation. Following this theory, these chondrogenic mediators could potentially stabilize or destabilize mRNA transcripts by influencing $R M R P$ levels and thus RNase MRP endoribonucleolytic capacity during chondrogenic development and cartilage disease. In $\mathrm{CHH}$, promoter mutations may result in inefficient transcription of the RMRP gene, whereas mutations in the transcribed region are thought to influence RNase MRP complex assembly, enzymatic activity, sub cellular localization, substrate recognition or RNA stability ${ }^{25}$. However, for cell function in general it remains to be determined whether transcriptional control of $R M R P$ transcription by (chondrogenic) morphogens is also present in other cells types and how this communicates with the RNA polymerase III transcription machinery ${ }^{26}$.

\subsubsection{Cyclin b2, an RNase MRP mRNA target, and its link to the chondrogenic mediator PTHrP}

One of the identified activities of the RNase MRP complex is endoribonucleolytic cleavage of the mRNA of $\mathrm{Clb2}$. Clb2 activates cyclin-dependent kinase-1 (CDK1) during M-phase (mitotic phase / nuclear cell division) ${ }^{27}$. In addition, anaphase-promoting complex (APC)-dependent proteolysis of $\mathrm{Clb2}$ is essential for mitotic exit ${ }^{28}$. Consequently, low Clb2 levels keep CDK1 activity to a minimum during GO phase (quiescence / resting phase) via APC-dependent proteolysis $^{29}$. It is thought that the terminal differentiation of hypertrophic chondrocytes is achieved via mitotic exit or entrance into G0 phase ${ }^{30}$. As RNase MRP activity has been shown to regulate $\mathrm{Clb} 2$ expression, it is tempting to speculate that the increase in $R M R P$ snoRNA levels during chondrocyte hypertrophy serves to decrease $\mathrm{Clb} 2$ protein levels by increasing the turnover of $\mathrm{Clb} 2$ mRNA and consequently inhibiting activation of CDK1. This hypothesis is substantiated by the observation by Saito and co-workers that CDK1 expression was found to be highly expressed in proliferative chondrocytes and was greatly diminished in hypertrophic chondrocytes in differentiating ATDC5 cultures and mouse growth plates ${ }^{31}$. In chapter $\mathbf{2}$ we therefore speculated that in a healthy growth plate, increased RMRP RNA levels in the hypertrophic zone may lead to reduced Clb2 levels, thereby hampering CDK1 activity and inducing mitotic arrest. Interestingly, in the same 
paper, Saito et al. ${ }^{31}$ also showed that the proliferation-promoting and hypertrophysuppressive action of PTHrP is CDK1-dependent. In agreement with this observation, in chapter 2, we show that Rmrp RNA levels are down regulated upon PTHrP exposure, presumably via decreased $R m r p$ promoter transcriptional activity ${ }^{11}$. This may lead to elevated Clb2 levels, thereby potentially aiding downstream CDK1 activity. This is further substantiated by our observation that knockdown of Rmrp RNA levels during ATDC5 chondrogenic differentiation increases $\mathrm{Clb2}$ and Pthrp mRNA levels and deregulates chondrogenic differentiation in an overall hypertrophy-suppressing fashion ${ }^{11}$. Lastly, considering our observations that the expression of PTHrP is induced during knockdown of Rmrp RNA and during chondrogenic differentiation of $\mathrm{CHH}$ fibroblasts, and taking into account the inhibiting action of PTHrP on RMRP promoter activity and RMRP RNA expression; it is noteworthy that disrupted PTH/PTHrP signaling has shown to inhibit proper skeletal development by impacting endochondral ossification ${ }^{32}$. Recently it has been shown that $\mathrm{CHH}$ fibroblasts have defects in cell cycle progression at $\mathrm{G}^{33}$. Consequently, it is tempting to speculate on a role for RMRP RNA in cell cycle control via a PTH/PTHrPdependent mechanism and it would be worthwhile to consider potential molecular interactions between RMRP RNA and PTH/PTHrP in skeletal dysplasias such as cartilage-hair hypoplasia, metaphyseal chondrodysplasia Jansen type (OMIM \#156400) ${ }^{34}$ and Blomstrand type chondrodysplasia (OMIM \#215045) 35 .

\subsubsection{RNase MRP mRNA target viperin}

In Chapter 3 we elucidated that the mRNA expression of RNase MRP / RMRP snoRNA target viperin was detectable early in ATDC5 and hBMSC chondrogenic differentiation and was highly induced at days 5-7 of differentiation ${ }^{12}$. In embryonal growth plates, various levels of viperin expression could be observed, depending of the spatiotemporal location in the growth plate. Viperin was originally discovered as an interferon-inducible protein, suggesting that induction of viperin expression during chondrogenic differentiation may be driven by interferon-related signaling activity, a relatively unexplored area of research. IFN- $\gamma$ has been demonstrated to inhibit transcription of the COL2A1 gene ${ }^{36,37}$ in mature chondrocytes and STAT1 expression was previously found specifically induced at day 7 in 
ATDC5 chondrogenic differentiation ${ }^{38}$, which would support the theory of an interferon signaling response as explanation for viperin induction at days 5-7. Until now it remained unexplored whether alterations in viperin levels could influence cellular differentiation processes. We found that in chondrogenic differentiation viperin stimulates the rate of protein secretion and of CXCL10 in particular. In line with a viperin-mediated interferon response during chondrogenic differentiation, the CXCL10 we detected in our LC-MS/MS analyses is an interferon-inducible chemokine. Interestingly, platelet-derived growth factor subunit $A$ was also found differentially expressed in the viperin overexpression secretome and platelet-derived growth factor has been described to synergistically act with IFN- $\gamma$ to induce CXCL10 expression in blood-derived macrophages ${ }^{39}$. Our data demonstrated that CXCL10 inhibits chondrogenic differentiation and TGF- $\beta$ signaling, which is in line with the inhibitory action of viperin-overexpression conditioned medium on chondrogenic differentiation.

It remains to be investigated why chondrogenic differentiation is regulated via viperin and how this, in turn, is regulated by RNase MRP / RMRP snoRNA. We hypothesized that control over the differentiation signals required for the early differentiation phase may be necessary to enable chromatin remodeling and coordinated transcriptomic reprogramming of the cell, before it can adopt a fully differentiated phenotype. We expect that viperindependent CXCL10 secretion may aid in this by antagonizing TGF- $\beta$ signaling. In that regard, we observed that CXCL10 inhibits phosphorylation of SMAD2/3, providing a potential mechanistic link between viperin-dependent CXCL10 secretion and TGF- $\beta$-driven chondrogenic differentiation. In addition, cross-talk between IFN- $\gamma$ and TGF- $\beta$ signaling, where IFN- $\gamma$-dependent STAT1 activity antagonizes SMAD3-dependent TGF- $\beta$ signaling, has been identified as well ${ }^{40}$. With the increasing number of identified RNase MRP targets and their general importance for the process of chondrogenic differentiation, dissecting their individual RNase MRP-driven regulation will prove to be an interesting challenge. In addition, it is not likely that all aspects of $\mathrm{CHH}$ pathobiology are caused by defective processing of one specific substrate. Instead, we believe that defective processing of one or the other specific RNase MRP substrate RNA will have different implications for different tissue/cell types. CHH-related defective processing of viperin mRNA leads to aberrant viperin levels in chondrocytic cells as shown Chapter $3^{12}$. Viperin ${ }^{41}$ and CXCL10 ${ }^{42}$ have been implicated in T-cell function, and in a microarray mRNA profiling study viperin was identified 
as one of the highest differentially expressed genes up-regulated in $\mathrm{CHH}$ leukocytes ${ }^{43}$, together with an increase of other interferon-related genes. Because $\mathrm{CHH}$ also presents with defective immunity caused by T-cell deficiency ${ }^{44}$, it is tempting to speculate that interferonrelated signaling through viperin and via CXCL10 is an important aspect of the molecular mechanism leading to growth plate and T-cell defects observed in $\mathrm{CHH}$.

\subsubsection{Yet unexplored RNase MRP mRNA targets?}

Thus far, we have observed that RMRP snoRNA levels are decreased in $\mathrm{CHH}$, which is accompanied by increased levels of viperin mRNA. In the previous paragraphs we were able to further elucidate the molecular mechanisms behind these observations. We hypothesize that by controlling the cellular RNase MRP levels, the cell has control over viperin and maybe other relevant target mRNA levels. There are indications that there may be additional protein subunits that interact with the protein subunits of the RNase MRP complex ${ }^{45}$. These may be involved in tuning the enzymatic activity of RNase MRP or recruit specific mRNAs, potentially controlling the RNase MRP's mRNA cleaving activity. There is evidence that RMRP RNA and the telomerase-associated reverse transcriptase (TERT) protein are able to form a complex that exerts RNA-dependent RNA polymerase activity, which is able to generate a double stranded RMRP RNA molecule ${ }^{46}$. Via a Dicer-dependent route siRNAs are generated from this molecule, which down-regulate cellular RMRP levels, as well as specific mRNAs ${ }^{47}$. RMRP RNA is the source of at least two other short RNAs designated RMRP-S1 and RMRP-S2, which function as miRNAs and have gene-silencing activity relevant for human cartilage-hair hypoplasia $(\mathrm{CHH})^{48}$. They potentially target genes relevant for skeletal development like SOX4, PTCH2 and BMPR2 and are thus expected to influence the process of chondrogenic differentiation that is affected in $\mathrm{CHH}$. In addition, there are indications that RMRP RNA could be involved in yet unexplored mRNA cleavage events. In the study by Mattijssen et al. ${ }^{10}$, where viperin mRNA was identified as a novel target of RNase MRP, a whole series of other mRNAs potentially under control of RNase MRP were identified as well. These include SYPL1 (involved in transport / synaptic transmission), ARPC1a (involved in actin cytoskeleton organization), CASP7 (caspase-7 precursor), H3F3A (involved in nucleosome assembly), DDT (D-dopachrome decarboxylase), 


\section{Chapter 6}

CAPNS1 (postitive regulator of cell proliferation), NQO1 (NAD(P)H dehydrogenase 1), HMOX1 (involved in heme oxidation), IL8 (negative regulator of cell proliferation and involved in chondrocyte hypertrophy) and GCLM (negative regulator of apoptosis). Furthermore, potential RNase MRP mRNA targets were also identified by Hermanns et al. ${ }^{43}$, who found mRNAs that were up-regulated in the leukocytes of four $\mathrm{CHH}$ patients as compared to healthy controls in two independent microarray studies ${ }^{43}$. The mRNAs, which were more than two-fold up-regulated in $\mathrm{CHH}$ patients, played a role in the immune system, cell cycle regulation (either via cell growth or apoptosis) and signal transduction. It should be noted that the effect of the reduced RMRP snoRNA levels observed in $\mathrm{CHH}$ on the patients' leukocyte mRNA expression could still be an indirect effect, which would need to be verified in biochemical assays similar to the work performed by Mattijssen et al. ${ }^{10}$ for viperin mRNA. It would be of interest to explore the above-mentioned mRNAs as potential novel downstream RNase MRP mRNA targets and to further elucidate their role in cell (chondrocyte) function, homeostasis, and more specifically the relationships between the diverse functions of RMRP RNA and their relevance for $\mathrm{CHH}$.

\section{2 mRNA targets of box C/D and box H/ACA snoRNAs}

Originally identified as guide RNAs for rRNA post-transcriptional modification, some canonical snoRNAs have been identified that modify mRNAs $s^{6,49}$. For example, candidate guide snoRNAs have been discovered that are predicted to 2'O-ribose methylate mRNAs in the human brain ${ }^{50}$. Brain-specific snoRNAs SNORD115 and SNORD116 have been shown to be processed into smaller RNAs that can affect the outcome of alternative splicing of various pre-mRNAs, by preventing the formation of particular pre-mRNA splicing variants ${ }^{51}$. Deficiency in paternal gene expression of these snoRNAs is implicated in the neurogenetic disease Prader-Willi syndrome ${ }^{50}$. SNORD115 is also involved in the regulation of serotonin receptor 5-HT2CR mRNA levels in brain cells, through alternative splicing and control of posttranscriptional nucleotide modification (adenosine-to-inosine editing ${ }^{52}$ ) of the target mRNA ${ }^{53,54}$. High-throughput sequencing showed that SNORD115 and SNORD116, along with a number of other human snoRNAs, are able to generate smaller fragments ${ }^{55-57}$. Short fragments derived from SNORD88C were shown to have complementarity to fibroblast 
growth receptor-3 (FGFR-3) pre-mRNA, and were capable of suppressing FGFR-3 expression and translation through base pairing with pre-mRNA regulatory elements ${ }^{58,59}$. Processing of box H/ACA and box C/D snoRNAs generates fragments which are termed snoRNA-derived RNAs (sdRNAs). Several of these sdRNAs were found to interact with Ago family proteins, the key components of RNA-induced silencing complexes (RISC) ${ }^{60,61}$ and it was shown that a number of box C/D RNA-derived fragments exhibit miRNA-like activity ${ }^{62}$. This would explain a potential mechanism by which snoRNAs can serve as a source of short regulatory RNA species involved in the control of processing and translation of various mRNAs. A putative mRNA-specific pseudouridylation guide for trypanosome mRNAs has also been found ${ }^{63}$ and transcriptome-wide mapping has revealed widespread dynamic regulation of pseudouridylation of $\mathrm{mRNA}^{64}$. The latter study discovered hundreds of novel pseudouridylation sites in human and yeast $m R_{N A s}{ }^{64}$. It was uncovered which pertubing pseudouridine synthases (PUSs) modify each site. PUS target sequence features were identified with mRNA pseudouridylation depending on snoRNA-guided PUSs as well as snoRNA-independent PUS activity ${ }^{64}$. The evidence above implies that canonical box C/D and H/ACA snoRNAs could also exert actions during chondrogenic differentiation via posttranscriptional modification and processing of relevant mRNAs.

In the light of our ATDC5 small RNA sequencing data (Chapter 4), it is noteworthy that several orphan snoRNAs were identified (Table 1). So-called "orphan RNAs" lack complementarity to known RNA species ${ }^{65,66}$. It is conceivable that some orphan snoRNAs function in cellular processes other than RNA post-transcriptional modification. The latter could be identified by using prediction tools such as RNAsnoop ${ }^{6}$. In this respect it will be worthwhile to further investigate the relevance of the orphan snoRNA that were differentially expressed in our ATDC5 RNA sequencing data sets (Table 1 / Chapter 4). Identification of snoRNA-mediated control of mRNA post-transcriptional modification would add a novel level of mRNA post-transcriptional regulation complexity as function of a cellular differentiation processes and improve the overall knowledge of the targets of the increasing number of orphan snoRNAs ${ }^{67,68}$. 


\begin{tabular}{|c|c|c|c|c|}
\hline snoRNA & $\begin{array}{l}\text { Box } \\
\text { classification }\end{array}$ & RNA target & LogFC & $\begin{array}{l}\text { FDR-adjusted } \\
\text { p-value }\end{array}$ \\
\hline \multicolumn{5}{|c|}{$\Delta$ day $0-7$} \\
\hline SNORD101 & $C / D$ & Unknown / orphan & -1.51 & 0.006 \\
\hline SNORD23 & $C / D$ & Unknown / orphan & -2.27 & 0.013 \\
\hline SNORA73 & H/ACA & Unknown / orphan & -1.53 & 0.039 \\
\hline SNORD50B & $C / D$ & Unknown / orphan & 1.36 & 0.042 \\
\hline \multicolumn{5}{|c|}{$\Delta$ day $7-14$} \\
\hline SNORD3 & $C / D$ & Unknown / orphan & -2.20 & 0.020 \\
\hline \multicolumn{5}{|c|}{$\Delta$ day $0-14$} \\
\hline SNORD101 & $C / D$ & Unknown / orphan & -2.36 & $3.39 \mathrm{E}-05$ \\
\hline SNORD3B2 & $C / D$ & Unknown / orphan & -2.03 & 0.005 \\
\hline SNORD86 & $C / D$ & Unknown / orphan & -1.23 & 0.044 \\
\hline
\end{tabular}

Table 1. Differentially expressed orphan snoRNAs discovered during RNA sequencing of chondrogenic ATDC5 differentiation. Differentially expressed snoRNAs between three distinct phases of ATDC5 chondrogenic differentiation, where day 0 reflects progenitor cells, day 7 reflects chondrogenic cells and day 14 reflects hypertrophic / mineralizing cells. Only the differentially expressed data from Chapter $\mathbf{4}$ is shown for snoRNAs with unknown RNA targets, the so called orphan snoRNAs.

\section{3 rRNA targets of box C/D and box H/ACA snoRNAs and their role in chondrogenic development}

The cell differentiation process requires major changes in the cellular proteome to accommodate the desired cell specialization, for example by fine-tuning ribosome production and function, to meet the demand for protein synthesis during proliferation, development, differentiation, homeostasis and disease ${ }^{69,70}$. It is therefore hypothesized that the formation of the cartilaginous extracellular matrix during chondrogenic differentiation would also demand an additional proteomic effort. It was thus expected that the regulation of protein translation plays an important role in chondrogenic differentiation. In Chapter 4, for the first time we identified that rRNA levels differ according to the stage of chondrogenic differentiation. Specifically at day 7 in ATDC5 chondrogenic differentiation (the chondrogenic phase which is predominantly associated with extracellular matrix production $^{13,71}$ ) the rRNA expression levels were significantly increased. Moreover, total 
cellular translation capacity was also shown to reach its highest levels at day 7 in differentiation. Post-transcriptional modification of rRNAs is essential for the basic biogenesis and translational activity of the ribosome $\mathrm{e}^{72,73}$, and was shown to support rRNA structural stability ${ }^{74,75}$, ribosomal protein association ${ }^{76,77}$ and maturation of crucial ribosome functional regions like the peptidyl transferase center (PTC) ${ }^{78}$ and the decoding center ${ }^{79}$. The recent identification of sites of sub-stoichiometric 2'-O-methylation and pseudouridylation ${ }^{80}$ has overturned the long-standing dogma that all rRNA modifications are constitutively present on ribosomes and highlights rRNA nucleotide modifications as an important source of ribosomal heterogeneity. This implies that not all positions are fully modified at all times. While the mechanisms regulating partial modification are largely unknown, changes in the rRNA modification pattern have been observed in response to environmental changes, during development ${ }^{81}$, and in disease ${ }^{72,73}$. For example, in yeast, only $68 \%$ of ribosomes are methylated at position A100 of the 18S rRNA under standard laboratory growth conditions ${ }^{72}$. Together, this suggests that rRNA modifications may contribute to the cellular translational control.

While the currently known >200 unique rRNA post-transcriptional modifications are guided by an almost equal number of site-specific snoRNAs ${ }^{80,82,83}$, the core enzymatic activities that are required to perform these post-transcriptional modifications are carried out by fibrillarin (the methyltransferase) and dyskerin (the pseudouridylase) ${ }^{83}$. Simultaneously with the elevated rRNA levels at day 7 in ATDC5 chondrogenic differentiation, expression of fibrillarin and dyskerin reached their peak levels as well. Interestingly, alterations in fibrillarin and dyskerin expression have been demonstrated to provoke changes in ribosome translational characteristics. There is evidence for rRNA 2'O-methylation plasticity, where knockdown of fibrillarin caused a reduction in global protein translation, with a specific reduction in IRESdependent protein translation ${ }^{84}$. Similarly, ribosomes purified from dyskerin-depleted cells also showed altered translational fidelity and IRES-mediated translation ${ }^{85,86}$. Our observation that fibrillarin and dyskerin expression alters as a function of ATDC5 chondrogenic differentiation might be explained by an increased demand of global rRNA post-transcriptional modification capacity for the maturation of the newly synthesized rRNA pool required during ATDC5 chondrogenic differentiation. This hypothesis, and its impact for chondrogenic development, needs further experimentation in future work. 
In Chapter 4 we also established that, depending on the differentiation stage, the snoRNA expression landscape changes during ATDC5 chondrogenic differentiation. This indicates that the position of various rRNA post-transcriptional modifications may be adapting to the differentiation stage. There is limited literature reporting on the differential expression of snoRNAs in models for cell differentiation, and no known literature in the context of chondrogenic differentiation. In neural differentiation of embryonic stem cells, specific snoRNA species were found to be differentially expressed depending on the differentiation stage $^{87}$. In hematopoietic development, snoRNAs were differentially expressed in a lineage specific pattern ${ }^{88}$. SnoRNA species were also expressed in a differentiation stage-dependent manner during hepatic differentiation of induced pluripotent stem cells ${ }^{89}$. The role of individual snoRNAs in increasingly recognized and should be investigated into further detail. For example, a study from Higa-Nakamine et al. ${ }^{90}$ showed that knockdown of box C/D SnORNAs SNORD26, SNORD44 or SNORD78 results in developmental defects in zebrafish. Knockdown of only one of these specific snoRNAs caused embryonic lethality, decreased body size, underdevelopment of the jaw, incomplete yolk sac extensions and hind brain defects. In Chapter 4, apart from a number of differentially expressed non-canonical snoRNAs (like SNORD101, SNORD23, SNORA73, SNORD50B, SNORD3, SNORD86 and others) that are involved in processes not involving rRNA post-transcriptional modification, the differentiation stage-depend dynamics in canonical snoRNA expression predicts a significant degree of rRNA post-transcriptional modification regulation during cell differentiation. A large number of snoRNAs with differentiation stage-dependent expression dynamics in snoRNA expression during ATDC5 chondrogenic differentiation are guiding posttranscriptional modification of rRNA sites in critical ribosome regions like the decoding center, the peptidyl transferase center and E-site. For example, SNORA40 (modifying helix 27 in the 18S rRNA decoding center in yeast ${ }^{91}$ ), SNORD46 (modifying helix 69 in $28 \mathrm{~S}$ rRNA $^{92,93}$ ) and SNORD36C and SNORA31 (modifying helix 68 in the $28 \mathrm{~S}$ rRNA ribosome's Esite $\left.^{94}\right)$. A recent study from Hebras $^{81}$ indicated regulation of rRNA 2'O-ribose methylation and accompanying guide snoRNAs during mouse development and highlights the potential relevance of ribosome heterogeneity during cell development. In our ATDC5 chondrogenic differentiation model it remains to be determined whether the rRNA target sites of the differentially expressed snoRNAs are actually post-transcriptionally modified in a differentiation stage specific manner. With emerging technologies such as RiboMethSeq ${ }^{95}$ 
and PSI-Seq ${ }^{96}$ to map dynamics in rRNA post-transcriptional modifications ${ }^{6,80}$, we will be able to unveil the snoRNA-driven chondrogenic differentiation stage-specific posttranscriptional modification of rRNAs. Identification of such differentiation stage-specific rRNA post-transcriptional modification-based ribosome heterogeneity can then be followedup by functional ribosome analyses to determine the cellular impact of these modifications. Determination of translational capacity, translation modus (IRES/cap ${ }^{86}$ ), stop-codon readthrough activity, fidelity etc., should be able to shed light on the role of these rRNA posttranscriptional modifications for the chondrogenic differentiation process.

In conclusion, the work in this thesis shows that chondrogenic differentiation is associated with regulation of mechanisms involved in ribosome biogenesis and translation activity. Differentiation-phase specific expression of snoRNAs suggests that specific snoRNAs may modulate the chondrocyte's developing phenotype via an rRNA post-transcriptional modification-based ribosome heterogeneity mechanism. This potentially facilitates the observed dynamics in translational activity. Because alterations in the chondrocyte's cellular phenotype are considered one of the fundamental pathological processes observed in ageing and in osteoarthritis ${ }^{97-100}$, it is expected that canonical box C/D and box H/ACA snoRNAs could also be of interest in the light of osteoarthritis. Indeed in Chapter $\mathbf{5}$ we observed differential expression of snoRNAs in ageing and osteoarthritis and this will be further discussed in section 6.5 of this general discussion.

\subsection{Processing of pre-rRNAs targets by non-canonical snoRNAs RMRP snoRNA and U3 in chondrogenic development and disease}

One of the earliest identified roles of the RNase MRP complex is endoribonucleolytic maturation of $5.8 \mathrm{~S}$ rRNA by cleaving the $47 \mathrm{~S}$ pre-rRNA internal transcribed spacer 1 (ITS1) at the A3 site ${ }^{1,2}$. It is tempting to speculate that the RMRP snoRNA/RNase MRP-mediated contribution to the synthesis of the large ribosomal subunit in the growth plate may support the protein synthetic activity of the growth plate to produce protein-rich cartilaginous extracellular matrix. In support of data from Goldfarb which indicates that endoribonucleolytic cleavage of ITS1 contributes to pre-RNA maturation ${ }^{2}$, our RMRP snoRNA knockdown data from Chapter 2 show accumulation of an ITS1 pre-rRNA processing 


\section{Chapter 6}

intermediate, as well as reduced levels of mature $5.8 \mathrm{~S}$ and $18 \mathrm{~S}$ rRNA in ATDC5 ${ }^{11}$. Moreover, similar accumulation of an ITS1 processing intermediate was observed in differentiating $\mathrm{CHH}$ cells with pathological mutations in the RMRP snoRNA. Because chondrogenic differentiation of ATDC5 and the developing growth plate are associated with high proliferative capacity ${ }^{101}$ and increased synthesis of protein-rich ECM, it is conceivable that this alters the cellular demand for mature rRNAs required for ribosome biogenesis. This could potentially explain the changes in Rmrp RNA expression that are observed during chondrogenic differentiation and the altered chondrocyte phenotype that is observed following Rmrp RNA knockdown. This implies for the first time, that the developing chondrocyte regulates its pre-rRNA processing via RMRP and ITS1 processing.

We have shown that during chondrogenic development and disease the processing of prerRNAs can be influenced by the non-canonical RMRP snoRNA. Therefore, it was expected that rRNA targets can also be influenced by other non-canonical snoRNAs in cartilage diseases that are not directly related to development or that are non-congenital, such as the age-related development of osteoarthritis (OA). Chondrocytes in the articular cartilage must express appropriate genes to maintain cartilage tissue homeostasis. This has shown to be altered in osteoarthritis ${ }^{102}$. One facet of the aberrant gene expression in osteoarthritis is the replay of chondrogenic differentiation with the expression of genes associated with chondrocyte hypertrophy and thus leading to pathological endochondral ossification (i.e. matrix breakdown and mineralization) ${ }^{102}$. Supporting a potential role for other noncanonical snoRNAs in this process, we have generated preliminary data showing that nonOA human articular chondrocytes (HAC), when exposed to IL-1 $\beta$ to mimic osteoarthritis, show reduced levels of $18 \mathrm{~S}$ and 5.8S rRNA and increased levels of the ITS1 rRNA processing intermediate (pre-5.8S). This is accompanied by reduced levels the non-canonical snoRNAs RMRP and U3 (Figure 2). The observed increase in pre-18S rRNA is associated with a reduction in U3 snoRNA, the primary snoRNA involved in the processing of pre-18S rRNA. The increased pre-5.8S rRNA levels are in concert with a reduction of RMRP snoRNA expression. Similar observations were made in non-OA versus OA human articular chondrocytes (data not shown). For the first time this implicates the non-canonical snoRNAs U3 (which is required for $18 \mathrm{~S}$ rRNA processing) and RMRP snoRNA (which is required for ITS1 processing and maturation of 5.8S rRNA) in osteoarthritis. From observations made in the work described in this thesis (Chapter 2) we already know that transcriptional activity of 
the RMRP gene is influenced by factors also involved in OA development ${ }^{11}$. This indicates that regulation of RMRP and $\mathrm{U} 3$ levels in osteoarthritis may be an active process contributing to the chondrocyte phenotype observed in osteoarthritis. This would be an exciting area for further investigation.

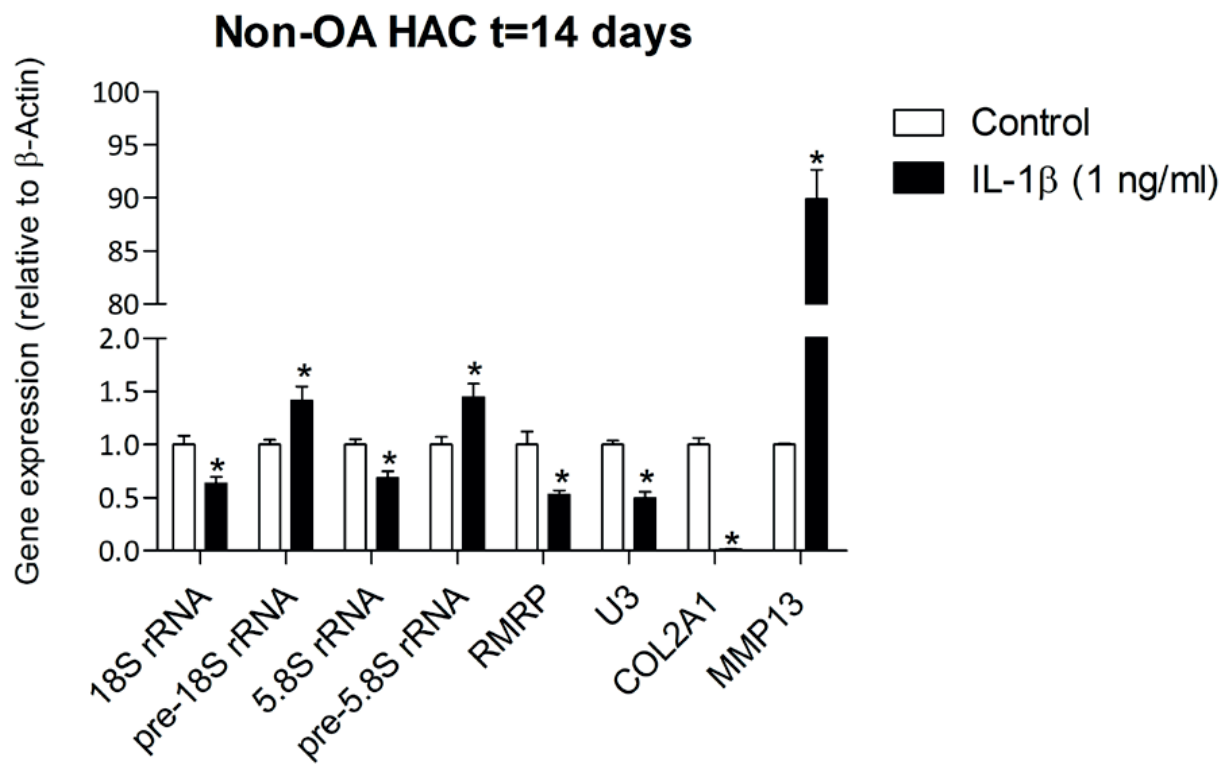

Figure 2. The role of non-canonical snoRNAs in osteoarthritis. Triplicate healthy human articular chondrocyte isolates were exposed to $1 \mathrm{ng} / \mathrm{ml}$ human IL-1 $1 \beta$ for 14 days. Medium was refreshed every other day, and real-time qPCR was performed on the indicated RNA species. Pre-18S rRNA corresponds to $5^{\prime}$ ETS cleavage site 1 and pre-5.8S corresponds to ITS- 1 cleavage site $2^{103}$. COL2A1 reduction (reduced chondrogenic capacity of HACs) and MMP13 increase (increased collagen matrix degradation of HACS) are indicative of the altered chondrogenic phenotype upon IL$1 \beta$ stimulation. Expression of healthy HACs was set to 1 and compared to IL-1 $\beta$-treated HACs using a two-way ANOVA with Bonferroni post-hoc correction, where * indicated $p<0.05$

\subsection{SnoRNAs as biomarkers for ageing and disease}

Previous studies have identified differentially expressed snoRNAs in cartilage ${ }^{104}$ and tendon ${ }^{105}$ ageing, and recently snoRNAs are emerging as novel non-invasive biomarkers for the diagnosis and prognosis of renal clear cell carcinoma ${ }^{106}$. However, in the context of 


\section{Chapter 6}

cartilage diseases, this has not yet been explored. During different stages of chondrogenic differentiation, snoRNAs are differentially expressed (Chapter 4). Like chondrogenic differentiation, osteoarthritis is characterized by different cartilage developmental stages $^{107,108}$. Therefore, it would be logical to investigate whether snoRNAs could be utilized as biomarkers for osteoarthritis. Through expression profiling of snoRNAs using deepsequencing we have revealed novel molecular features relating to joint ageing and osteoarthritis (Chapter $\mathbf{5})^{107}$. Whilst gene expression has been evaluated in animal models of osteoarthritis ${ }^{109-111}$, these were primarily investigating protein-coding genes. Furthermore, apart from the study conducted by Loeser et al. ${ }^{111}$, these experiments evaluated a single tissue, namely the articular cartilage. However, osteoarthritis is a pathology that involves the whole joint "as an organ" and diagnostics would preferably be based on non-invasive blood tests. Therefore we undertook our analyses on serum and on mouse joints as a whole (including cartilage, meniscus, subchondral bone and the joint capsule with synovium). To investigate the effects of joint ageing on snoRNA expression we used 8 month-old mice, which is equivalent to 25 to 28 years in humans and considered to be "young" and compared this with 24 month-old mice, which represented an "old" group ${ }^{112}$. To study the effect of post-traumatic osteoarthritis we measured snoRNAs expression in whole joints and in serum from 24 month-old mice following DMM (mouse model of osteoarthritis, achieved by destabilization of the medial meniscus ${ }^{113}$ ). SnoRNAs were previously identified in serum ${ }^{114-116}$ and are serum-stable in protein complexes ${ }^{114}$ or contained within extracellular vesicles in serum ${ }^{107}$. However, it should be considered that it is not yet clear whether disease-associated RNAs detected in the circulation result from local tissue disturbances and associated cell death by active local secretion via exosomes or microvesicles, or are a systemic response upon local tissue damage ${ }^{117-119}$. This may depend on the specific pathology and/or specific RNA species detected.

In the young versus old serum, more snoRNAs (27 snoRNAs) were significantly up-regulated compared to the old sham versus old DMM serum (18 snoRNAs). This indicated that ageing had a greater effect on differential snoRNA presence in serum compared to traumatic osteoarthritis. As we were studying the whole joint we were unable to determine the amount each tissue type within the joint contributed to the differential expression of snoRNAs. Each tissue will vary with regards to its cellularity and hence its RNA and snoRNAs content. Thus whilst a novel aspect of this study was that snoRNAs were extracted from the 
multiple tissues that form the joint, our approach may have been less sensitive in detecting snoRNAs changes related to a single tissue (or with a low cellularity). However, our approach did have the advantage of determining snoRNAs that could be more generally implicated in osteoarthritis and could thus serve as a biomarker. Despite these potential limitations, we identified a number of potentially interesting snoRNAs for future studies. For example, SNORA73 was increased in old joint and old serum and represents a potential joint 'biological ageing' marker ${ }^{107}$. A reliable measurement of the "state of ageing" and a prediction of the risk of the onset of age-related diseases such as osteoarthritis would be beneficial. Such a strategy could serve as a measure of an individual's "joint biological age" and potentially predict an age-related biological response more accurately than the person's chronological age. SNORA64 was increased and SNORD46 was decreased in DMM serum, but both were not differentially expressed in young versus old serum, highlighting these snoRNAs as potential osteoarthritis markers ${ }^{107}$. An interesting finding was an increase in SNORD38 in the ageing joint, considering that Zhang et al. ${ }^{114}$ demonstrated a strong association between serum levels of SNORD38 and severe cartilage damage following anterior cruciate ligament $(A C L)$ injury ${ }^{120}$. The most differentially expressed snoRNA in DMM serum was SNORD116. An increase that could also be confirmed in serum of horses with metacarpophalangeal ${ }^{121}$ osteoarthritis. Peffers et al. ${ }^{122}$ previously identified SNORD116 as increased in osteoarthritis compared to normal human cartilage in a micro-array study. A loss of SNORD116 entails a significant contribution to the etiology of the neurodegenerative genetic disease Prader-Willi syndrome ${ }^{123}$, a disorder resulting in developmental delay and obesity due to hyperphagia ${ }^{124}$. However, the clinical presentation also includes short stature and low bone mineral density ${ }^{125}$. Loss of the Prader-Willi syndrome critical genomic region (which includes SNORD116) in transgenic mice resulted in reduced bone mineral density, delayed skeletal development, reduced bone size and inhibition of osteoblast function ${ }^{126}$. Humans with osteoarthritis have an increased bone mineral density in the affected joints ${ }^{127}$. Thus our findings were able to elucidate a snoRNA serum biomarker with a potential role in the pathogenesis of osteoarthritis.

Overall, the results of our biomarker study implicate that snoRNAs in the future may be utilized as a biomarker to assess the condition of the articular joint. However additional investigations are needed to answer questions like: "Do snoRNAs in serum indeed reside in extracellular vesicles?". This is important because it would implicate that snoRNAs are 
Chapter 6

secreted as part of an active process. To further assess the prognostic value of the snoRNAs identified above, it would also be relevant to address whether these snoRNAs can be detected in human subjects with varying degrees of osteoarthritis.

\section{References chapters 6 and 7}

Specified below Chapter 7. 
General discussion 



\section{Chapter 7}

\section{An overview of proposed molecular interactions from this thesis}


Chapter 7 


\section{An overview of proposed molecular interactions from this thesis}

Throughout this thesis we have explored snoRNAs in the context of chondrogenic differentiation, $\mathrm{CHH}$ and osteoarthritis. In order to provide a comprehensive overview of the molecular interactions of snoRNAs in chondrogenic development and disease identified in this thesis, a scheme was generated which is presented in Figure 3. In bold the molecular interactions can be found that were newly identified as part of this thesis.

\subsection{ITS-1 pathway}

Recently it has been shown that human RMRP RNA catalyzes the endoribonucleolytic cleavage of ITS1, thereby contributing to pre-rRNA maturation ${ }^{2}$ (Figure 3 orange pathway). Indeed our ATDC5 Rmrp RNA knockdown data (Chapter 2) show accumulation of an ITS1 pre-rRNA processing intermediate as well as reduced levels of mature $5.8 \mathrm{~S}$ and $18 \mathrm{~S}$ rRNA ${ }^{11}$. A similar accumulation of an ITS1 processing intermediate was observed in differentiating chondrocytic $\mathrm{CHH}$ cells (Chapter 2) ${ }^{11}$. Chondrogenic differentiation of ATDC5 as well as growth plate development is associated with high proliferative capacity ${ }^{101}$ and increased synthesis of protein-rich extracellular matrix. This may alter the cellular demand for mature rRNAs required for ribosome biogenesis during chondrogenic differentiation and possibly explaining the dwarfism phenotype of $\mathrm{CHH}$ patients (Chapter 2$)^{11}$.

\subsection{RMRP miRNA pathway}

RMRP RNA is the source of at least two miRNAs designated RMRP-S1 and RMRP-S2, that were shown to possess gene-silencing activity relevant for human cartilage-hair hypoplasia $(\mathrm{CHH})^{48}$ (Figure 3 pink pathway). These miRNAs have been predicted to target genes relevant for skeletal development like SOX4, PTCH2 and BMPR2 and are thus interesting candidates for further investigation in the context of chondrogenic differentiation in $\mathrm{CHH}$. 


\subsection{Cyclin b2 pathway}

Tissues with fast-dividing cell types such as growth plates, hair follicles and bone marrow seem to be the most affected in $\mathrm{CHH}^{128}$, suggesting a role for RMRP RNA in cell cycle regulation. One of the identified activities of the RMRP RNA is endoribonucleolytic cleavage of the mRNA of cyclin b2 $(\mathrm{Clb} 2)^{9}$ (Figure 3 purple pathway). As explained in section 6.1.2, this B-type cyclin activates CDK1 during mitotic phase; and APC-dependent proteolysis of Clb2 is essential for mitotic exit ${ }^{28}$. In addition, low Clb2 levels keep CDK1 activity to a minimum during G0 (quiescence / resting) phase via similar APC-dependent proteolysis ${ }^{29}$. It is likely that the strictly regulated terminal differentiation of hypertrophic chondrocytes is achieved by mitotic exit or entrance into G0 phase. As RMRP RNA activity has been shown to degrade the Clb2 message, we speculated in Chapter 2 that the increase of RMRP RNA levels during chondrocyte hypertrophy serves to decrease $\mathrm{Clb} 2$ protein levels by increasing the turnover of $\mathrm{Cl} / \mathrm{b} 2 \mathrm{mRNA}$ and thus inhibiting activation of $\mathrm{CDK} 1^{11}$. Interestingly, the proproliferative, hypertrophy-suppressing actions of PTHrP were shown to be, at least in part, CDK1-dependent ${ }^{31}$. Indeed, in Chapter 2 we observed that Rmrp RNA levels are down regulated upon PTHrP exposure, presumably via decreased Rmrp promoter transcriptional activity $^{11}$. This may result in elevated Clb2 levels, thereby potentially supporting downstream PTHrP and CDK1 activity.

\subsection{Chondrogenic mediators}

In Chapter 2 we also found that bFGF, which, like PHTrP, delays chondrocyte hypertrophy and terminal differentiation ${ }^{13-15,129}$, downregulates $R m r p$ promoter transcriptional activity ${ }^{11}$ (Figure 3, blue pathway). Moreover, exposure of ATDC5 cells to dorsomorphin, an inhibitor of BMP-mediated SMAD1/5/8 phosphorylation ${ }^{21}$, greatly reduced Rmrp promoter activity, further supporting a potentially BMP-mediated control of RMRP transcription. In line with a potential feedback mechanism it is notable that BMPR2 (the type II BMP receptor) is a potential target of $R M R P$-S2, the RMRP RNA-derived siRNA ${ }^{48}$ (link from blue to pink pathway, Figure 3). 


\subsection{Viperin pathway}

RNase MRP is able to cleave viperin $\mathrm{mRNA}^{10}$. In $\mathrm{CHH}, R M R P$ levels are reduced, resulting in increased expression of viperin mRNA (Figure $\mathbf{3}$ green pathway). In Chapter 3, we show that in ATDC5 cells viperin overexpression increases overall protein secretion ${ }^{12}$ and CXCL10 in particular. We also show that during overexpression of viperin TGF- $\beta /$ SMAD2/3 activity is reduced and that this TGF- $B /$ SMAD3 activity is controlled by the viperin secretome and $\mathrm{CXCL10}{ }^{12}$. Moreover, viperin overexpression reduces overall chondrogenic differentiation. Lastly, we showed that the viperin-CXCL10-TGF- $\beta /$ SMAD2/3 axis is deregulated in chondrocytic $\mathrm{CHH}$ cells, possibly explaining the $\mathrm{CHH}$ phenotype. Interestingly, both Chapter 2 and Chapter 3 point towards a role for TGF- $\beta / S M A D$ signaling in the pathobiology of $\mathrm{CHH}$.

\subsection{What about other snoRNAs?}

$R M R P$ RNA is just one of approximately 200 snoRNAs ${ }^{82}$. The majority of snoRNAs can be subdivided into two classes with distinctive, evolutionary conserved sequence elements: the box $C / D$ and box H/ACA snoRNAs. Based on sequence complementarity with their target rRNA, these classes guide the post-transcriptional 2'O-ribose methylation and pseudouridylation (respectively) of specific rRNA nucleotides ${ }^{130,131}$. In Chapter 4 we show that there is differential expression of snoRNAs during distinct phases of ATDC5 chondrogenic differentiation (see Figure $\mathbf{3}$ grey pathway). Since these snoRNAs are involved in fine-tuning ribosome translational activity and characteristics, we speculate that these SnoRNAs facilitate cellular translational activities that are key for the chondrogenic differentiation process, but also for osteoarthritis development. In Chapter $\mathbf{5}$ we show that snoRNAs are differentially expressed during ageing and in the osteoarthritic joint ${ }^{107}$. In addition we show that serum snoRNAs could be considered as potential biomarkers for joint ageing and post-traumatic osteoarthritis. 


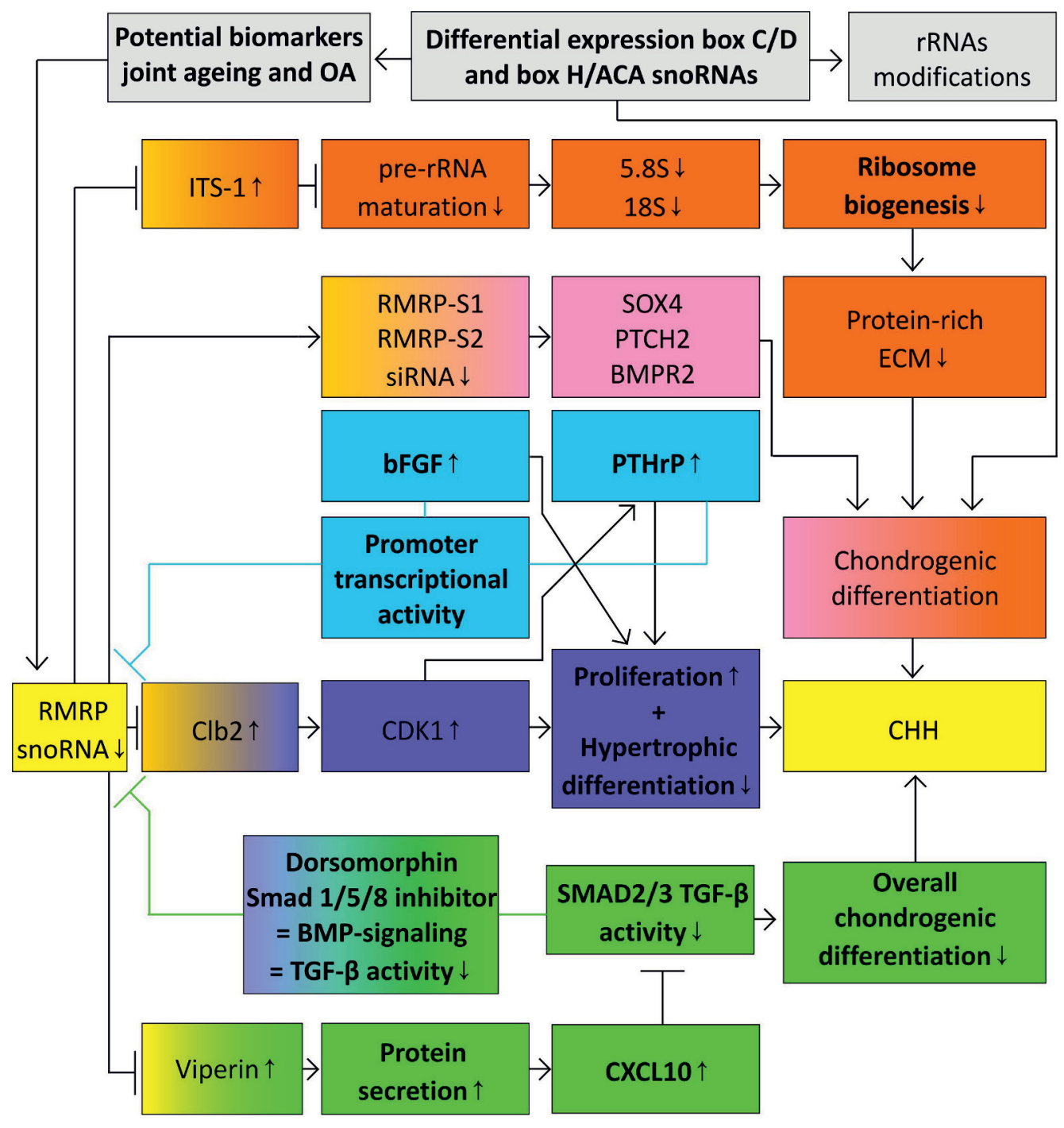

Figure 3. Proposed scheme of how RMRP snoRNA-derived functions and box $C / D$ and $H / A C A$ snoRNA functions can be linked to chondrogenic differentiation, $\mathrm{CHH}$ and osteoarthritis, with in bold the interactions that were newly identified as part of this thesis. See section 7.1 to 7.6 for a more elaborate description. 


\section{Concluding words}

The work presented in this thesis provides directions for pathways in which snoRNAs function in chondrocyte development and disease, and I hope the results from this thesis will inspire other researchers to follow them up and extrapolate and expand these findings to different cell types, tissues and developmental contexts. 


\section{References chapters 6 and 7}

1 Lygerou, Z., Allmang, C., Tollervey, D. \& Seraphin, B. Accurate processing of a eukaryotic precursor ribosomal RNA by ribonuclease MRP in vitro. Science 272, 268270 (1996).

2 Goldfarb, K. C. \& Cech, T. R. Targeted CRISPR disruption reveals a role for RNase MRP RNA in human preribosomal RNA processing. Genes Dev 31, 59-71, doi:10.1101/gad.286963.116 (2017).

3 Burman, L. G. \& Mauro, V. P. Analysis of rRNA processing and translation in mammalian cells using a synthetic 18S rRNA expression system. Nucleic Acids Res 40, 8085-8098, doi:10.1093/nar/gks530 (2012).

4 Filipowicz, W. \& Pogacic, V. Biogenesis of small nucleolar ribonucleoproteins. Curr Opin Cell Biol 14, 319-327 (2002).

5 Matera, A. G., Terns, R. M. \& Terns, M. P. Non-coding RNAs: lessons from the small nuclear and small nucleolar RNAs. Nat Rev Mol Cell Biol 8, 209-220, doi:10.1038/nrm2124 (2007).

Tafer, H., Kehr, S., Hertel, J., Hofacker, I. L. \& Stadler, P. F. RNAsnoop: efficient target prediction for H/ACA snoRNAs. Bioinformatics 26, 610-616, doi:10.1093/bioinformatics/btp680 (2010).

7 Welting, T. J., van Venrooij, W. J. \& Pruijn, G. J. Mutual interactions between subunits of the human RNase MRP ribonucleoprotein complex. Nucleic Acids Res 32, 2138-2146, doi:10.1093/nar/gkh539 (2004).

8 Chang, D. D. \& Clayton, D. A. A novel endoribonuclease cleaves at a priming site of mouse mitochondrial DNA replication. EMBO J 6, 409-417 (1987).

9 Gill, T., Cai, T., Aulds, J., Wierzbicki, S. \& Schmitt, M. E. RNase MRP cleaves the CLB2 mRNA to promote cell cycle progression: novel method of mRNA degradation. Mol Cell Biol 24, 945-953 (2004).

Mattijssen, S. et al. Viperin mRNA is a novel target for the human RNase MRP/RNase P endoribonuclease. Cell Mol Life Sci 68, 2469-2480, doi:10.1007/s00018-010-0568-3 (2011).

11 Steinbusch, M. M. F. et al. Expression of RMRP RNA is regulated in chondrocyte hypertrophy and determines chondrogenic differentiation. Sci Rep 7, 6440, doi:10.1038/s41598-017-06809-5 (2017).

12 Steinbusch, M. M. F. et al. The antiviral protein viperin regulates chondrogenic differentiation via CXCL10 protein secretion. J Biol Chem 294, 5121-5136, doi:10.1074/jbc.RA119.007356 (2019).

13 Kronenberg, H. M. Developmental regulation of the growth plate. Nature 423, 332336, doi:10.1038/nature01657 (2003).

14 Guo, J. et al. PTH/PTHrP receptor delays chondrocyte hypertrophy via both Runx2dependent and -independent pathways. Dev Biol 292, 116-128, doi:10.1016/j.ydbio.2005.12.044 (2006). 
Terkeltaub, R. A. et al. Bone morphogenetic proteins and bFGF exert opposing regulatory effects on PTHrP expression and inorganic pyrophosphate elaboration in immortalized murine endochondral hypertrophic chondrocytes (MCT cells). J Bone Miner Res 13, 931-941, doi:10.1359/jbmr.1998.13.6.931 (1998).

Cals, F. L., Hellingman, C. A., Koevoet, W., Baatenburg de Jong, R. J. \& van Osch, G. J. Effects of transforming growth factor-beta subtypes on in vitro cartilage production and mineralization of human bone marrow stromal-derived mesenchymal stem cells. J Tissue Eng Regen Med 6, 68-76, doi:10.1002/term.399 (2012).

Wang, W., Rigueur, D. \& Lyons, K. M. TGFbeta signaling in cartilage development and maintenance. Birth Defects Res C Embryo Today 102, 37-51, doi:10.1002/bdrc.21058 (2014).

8 Xu, Y., James, A. W. \& Longaker, M. T. Transforming growth factor-beta1 stimulates chondrogenic differentiation of posterofrontal suture-derived mesenchymal cells in vitro. Plast Reconstr Surg 122, 1649-1659, doi:10.1097/PRS.0b013e31818cbf44 (2008).

$\mathrm{Wu}$, J. et al. Mechanism and in vitro pharmacology of TAK1 inhibition by (5Z)-7Oxozeaenol. ACS Chem Biol 8, 643-650, doi:10.1021/cb3005897 (2013).

Caron, M. M. et al. Hypertrophic differentiation during chondrogenic differentiation of progenitor cells is stimulated by BMP-2 but suppressed by BMP-7. Osteoarthritis Cartilage 21, 604-613, doi:10.1016/j.joca.2013.01.009 (2013).

1 Sanvitale, C. E. et al. A new class of small molecule inhibitor of BMP signaling. PLoS One 8, e62721, doi:10.1371/journal.pone.0062721 (2013).

2 Studer, D., Millan, C., Ozturk, E., Maniura-Weber, K. \& Zenobi-Wong, M. Molecular and biophysical mechanisms regulating hypertrophic differentiation in chondrocytes and mesenchymal stem cells. Eur Cell Mater 24, 118-135; discussion 135 (2012).

23 Yuan, Y. \& Reddy, R. 5' flanking sequences of human MRP/7-2 RNA gene are required and sufficient for the transcription by RNA polymerase III. Biochim Biophys Acta 1089, 33-39, doi:10.1016/0167-4781(91)90081-v (1991).

4 Park, J. \& Jeong, S. Wnt activated beta-catenin and YAP proteins enhance the expression of non-coding RNA component of RNase MRP in colon cancer cells. Oncotarget 6, 34658-34668, doi:10.18632/oncotarget.5778 (2015).

5 Mattijssen, S., Welting, T. J. \& Pruijn, G. J. RNase MRP and disease. Wiley Interdiscip Rev RNA 1, 102-116, doi:10.1002/wrna.9 (2010).

6 Orioli, A., Pascali, C., Pagano, A., Teichmann, M. \& Dieci, G. RNA polymerase III transcription control elements: themes and variations. Gene 493, 185-194, doi:10.1016/j.gene.2011.06.015 (2012).

27 Enserink, J. M. \& Kolodner, R. D. An overview of Cdk1-controlled targets and processes. Cell Div 5, 11, doi:10.1186/1747-1028-5-11 (2010).

Wasch, R. \& Cross, F. R. APC-dependent proteolysis of the mitotic cyclin Clb2 is essential for mitotic exit. Nature 418, 556-562, doi:10.1038/nature00856 (2002). 
Guardavaccaro, D. \& Pagano, M. Stabilizers and destabilizers controlling cell cycle oscillators. Mol Cell 22, 1-4, doi:10.1016/j.molcel.2006.03.017 (2006).

30 Shang, $X$. et al. Notch signaling indirectly promotes chondrocyte hypertrophy via regulation of BMP signaling and cell cycle arrest. Sci Rep 6, 25594, doi:10.1038/srep25594 (2016).

31 Saito, M. et al. The Indispensable Role of Cyclin-Dependent Kinase 1 in Skeletal Development. Sci Rep 6, 20622, doi:10.1038/srep20622 (2016).

32 Schipani, E. \& Provot, S. PTHrP, PTH, and the PTH/PTHrP receptor in endochondral bone development. Birth Defects Res C Embryo Today 69, 352-362, doi:10.1002/bdrc.10028 (2003).

33 Vakkilainen, S. et al. The human long non-coding RNA gene RMRP has pleiotropic effects and regulates cell-cycle progression at G2. Sci Rep 9, 13758, doi:10.1038/s41598-019-50334-6 (2019).

34 Savoldi, G. et al. Prenatal presentation and postnatal evolution of a patient with Jansen metaphyseal dysplasia with a novel missense mutation in PTH1R. Am J Med Genet A 161A, 2614-2619, doi:10.1002/ajmg.a.36115 (2013).

35 Jobert, A. S. et al. Absence of functional receptors for parathyroid hormone and parathyroid hormone-related peptide in Blomstrand chondrodysplasia. J Clin Invest 102, 34-40, doi:10.1172/JCI2918 (1998).

36 Osaki, M. et al. The TATA-containing core promoter of the type II collagen gene (COL2A1) is the target of interferon-gamma-mediated inhibition in human chondrocytes: requirement for Stat1 alpha, Jak1 and Jak2. Biochem J 369, 103-115, doi:10.1042/BJ20020928 (2003).

37 Goldring, M. B., Fukuo, K., Birkhead, J. R., Dudek, E. \& Sandell, L. J. Transcriptional suppression by interleukin-1 and interferon-gamma of type II collagen gene expression in human chondrocytes. J Cell Biochem 54, 85-99, doi:10.1002/jcb.240540110 (1994).

38 Morimoto, H., Baba, R., Haneji, T. \& Doi, Y. Double-stranded RNA-dependent protein kinase regulates insulin-stimulated chondrogenesis in mouse clonal chondrogenic cells, ATDC-5. Cell Tissue Res 351, 41-47, doi:10.1007/s00441-012-1521-6 (2013).

39 Dhillon, N. K., Peng, F., Ransohoff, R. M. \& Buch, S. PDGF synergistically enhances IFN-gamma-induced expression of CXCL10 in blood-derived macrophages: implications for HIV dementia. J Immunol 179, 2722-2730, doi:10.4049/jimmunol.179.5.2722 (2007).

40 Ulloa, L., Doody, J. \& Massague, J. Inhibition of transforming growth factorbeta/SMAD signalling by the interferon-gamma/STAT pathway. Nature 397, 710-713, doi:10.1038/17826 (1999).

41 Qiu, L. Q., Cresswell, P. \& Chin, K. C. Viperin is required for optimal Th2 responses and T-cell receptor-mediated activation of NF-kappaB and AP-1. Blood 113, 35203529, doi:10.1182/blood-2008-07-171942 (2009). 
42 Groom, J. R. \& Luster, A. D. CXCR3 in T cell function. Exp Cell Res 317, 620-631, doi:10.1016/j.yexcr.2010.12.017 (2011).

43 Hermanns, P. et al. Consequences of mutations in the non-coding RMRP RNA in cartilage-hair hypoplasia. Hum Mol Genet 14, 3723-3740, doi:10.1093/hmg/ddi403 (2005).

44 Makitie, O., Kaitila, I. \& Savilahti, E. Susceptibility to infections and in vitro immune functions in cartilage-hair hypoplasia. Eur $J$ Pediatr 157, 816-820, doi:10.1007/s004310050943 (1998).

45 Jiang, T. \& Altman, S. Protein-protein interactions with subunits of human nuclear RNase P. Proc Natl Acad Sci U S A 98, 920-925, doi:10.1073/pnas.021561498 (2001).

46 Maida, Y. et al. An RNA-dependent RNA polymerase formed by TERT and the RMRP RNA. Nature 461, 230-235, doi:10.1038/nature08283 (2009).

47 Maida, Y., Kyo, S., Lassmann, T., Hayashizaki, Y. \& Masutomi, K. Off-target effect of endogenous siRNA derived from RMRP in human cells. Int J Mol Sci 14, 9305-9318, doi:10.3390/ijms14059305 (2013).

48 Rogler, L. E. et al. Small RNAs derived from IncRNA RNase MRP have gene-silencing activity relevant to human cartilage-hair hypoplasia. Hum Mol Genet 23, 368-382, doi:10.1093/hmg/ddt427 (2014).

49 Bachellerie, J. P., Cavaille, J. \& Huttenhofer, A. The expanding snoRNA world. Biochimie 84, 775-790 (2002).

50 Cavaille, J. et al. Identification of brain-specific and imprinted small nucleolar RNA genes exhibiting an unusual genomic organization. Proc Natl Acad Sci U S A 97, 14311-14316, doi:10.1073/pnas.250426397 (2000).

51 Kishore, S. et al. The snoRNA MBII-52 (SNORD 115) is processed into smaller RNAs and regulates alternative splicing. Hum Mol Genet 19, 1153-1164, doi:10.1093/hmg/ddp585 (2010).

52 Zinshteyn, B. \& Nishikura, K. Adenosine-to-inosine RNA editing. Wiley Interdiscip Rev Syst Biol Med 1, 202-209, doi:10.1002/wsbm.10 (2009).

53 Kishore, S. \& Stamm, S. The snoRNA HBII-52 regulates alternative splicing of the serotonin receptor 2C. Science 311, 230-232, doi:10.1126/science.1118265 (2006).

54 Vitali, P. et al. ADAR2-mediated editing of RNA substrates in the nucleolus is inhibited by C/D small nucleolar RNAs. J Cell Biol 169, 745-753, doi:10.1083/jcb.200411129 (2005).

55 Taft, R. J. et al. Small RNAs derived from snoRNAs. RNA 15, 1233-1240, doi:10.1261/rna.1528909 (2009).

56 Shen, M. et al. Direct cloning of double-stranded RNAs from RNase protection analysis reveals processing patterns of C/D box snoRNAs and provides evidence for widespread antisense transcript expression. Nucleic Acids Res 39, 9720-9730, doi:10.1093/nar/gkr684 (2011). 
57 Falaleeva, M. \& Stamm, S. Processing of snoRNAs as a new source of regulatory noncoding RNAs: snoRNA fragments form a new class of functional RNAs. Bioessays 35, 46-54, doi:10.1002/bies.201200117 (2013).

58 Ono, M. et al. Identification of human miRNA precursors that resemble box C/D snoRNAs. Nucleic Acids Res 39, 3879-3891, doi:10.1093/nar/gkq1355 (2011).

59 Ono, M. et al. Analysis of human small nucleolar RNAs (snoRNA) and the development of snoRNA modulator of gene expression vectors. Mol Biol Cell 21, 1569-1584, doi:10.1091/mbc.E10-01-0078 (2010).

60 Ender, C. et al. A human snoRNA with microRNA-like functions. Mol Cell 32, 519-528, doi:10.1016/j.molcel.2008.10.017 (2008).

61 Burroughs, A. M. et al. Deep-sequencing of human Argonaute-associated small RNAs provides insight into miRNA sorting and reveals Argonaute association with RNA fragments of diverse origin. RNA Biol 8, 158-177, doi:10.4161/rna.8.1.14300 (2011).

Brameier, M., Herwig, A., Reinhardt, R., Walter, L. \& Gruber, J. Human box C/D snoRNAs with miRNA like functions: expanding the range of regulatory RNAs. Nucleic Acids Res 39, 675-686, doi:10.1093/nar/gkq776 (2011).

Liang, X. H., Xu, Y. X. \& Michaeli, S. The spliced leader-associated RNA is a trypanosome-specific sn(o) RNA that has the potential to guide pseudouridine formation on the SL RNA. RNA 8, 237-246 (2002).

64 Schwartz, S. et al. Transcriptome-wide mapping reveals widespread dynamicregulated pseudouridylation of ncRNA and mRNA. Cell 159, 148-162, doi:10.1016/j.cell.2014.08.028 (2014).

Huttenhofer, A. et al. RNomics: an experimental approach that identifies 201 candidates for novel, small, non-messenger RNAs in mouse. EMBO J 20, 2943-2953, doi:10.1093/emboj/20.11.2943 (2001).

Kiss, T. Small nucleolar RNA-guided post-transcriptional modification of cellular RNAs. EMBO J 20, 3617-3622, doi:10.1093/emboj/20.14.3617 (2001).

Jady, B. E. \& Kiss, T. Characterisation of the U83 and U84 small nucleolar RNAs: two novel 2'-O-ribose methylation guide RNAs that lack complementarities to ribosomal RNAs. Nucleic Acids Res 28, 1348-1354 (2000).

Li, S. G., Zhou, H., Luo, Y. P., Zhang, P. \& Qu, L. H. Identification and functional analysis of 20 Box H/ACA small nucleolar RNAs (snoRNAs) from Schizosaccharomyces pombe. J Biol Chem 280, 16446-16455, doi:10.1074/jbc.M500326200 (2005).

Sanchez, C. G. et al. Regulation of Ribosome Biogenesis and Protein Synthesis Controls Germline Stem Cell Differentiation. Cell Stem Cell 18, 276-290, doi:10.1016/j.stem.2015.11.004 (2016).

70 Vanzi, F., Vladimirov, S., Knudsen, C. R., Goldman, Y. E. \& Cooperman, B. S. Protein synthesis by single ribosomes. RNA 9, 1174-1179, doi:10.1261/rna.5800303 (2003).

71 Yao, Y. \& Wang, Y. ATDC5: an excellent in vitro model cell line for skeletal development. J Cell Biochem 114, 1223-1229, doi:10.1002/jcb.24467 (2013). 
72 Lafontaine, D. L. Noncoding RNAs in eukaryotic ribosome biogenesis and function. Nat Struct Mol Biol 22, 11-19, doi:10.1038/nsmb.2939 (2015).

Sloan, K. E. et al. Tuning the ribosome: The influence of rRNA modification on eukaryotic ribosome biogenesis and function. RNA Biol 14, 1138-1152, doi:10.1080/15476286.2016.1259781 (2017).

74 King, T. H., Liu, B., McCully, R. R. \& Fournier, M. J. Ribosome structure and activity are altered in cells lacking snoRNPs that form pseudouridines in the peptidyl transferase center. Mol Cell 11, 425-435, doi:10.1016/s1097-2765(03)00040-6 (2003).

75 Baxter-Roshek, J. L., Petrov, A. N. \& Dinman, J. D. Optimization of ribosome structure and function by rRNA base modification. PLoS One 2, e174, doi:10.1371/journal.pone.0000174 (2007).

76 Sharma, S. \& Lafontaine, D. L. J. 'View From A Bridge': A New Perspective on Eukaryotic rRNA Base Modification. Trends Biochem Sci 40, 560-575, doi:10.1016/j.tibs.2015.07.008 (2015).

77 Behrmann, E. et al. Structural snapshots of actively translating human ribosomes. Cell 161, 845-857, doi:10.1016/j.cell.2015.03.052 (2015).

78 Polacek, N. \& Mankin, A. S. The ribosomal peptidyl transferase center: structure, function, evolution, inhibition. Crit Rev Biochem Mol Biol 40, 285-311, doi:10.1080/10409230500326334 (2005).

79 Liang, X. H., Liu, Q. \& Fournier, M. J. Loss of rRNA modifications in the decoding center of the ribosome impairs translation and strongly delays pre-rRNA processing. RNA 15, 1716-1728, doi:10.1261/rna.1724409 (2009).

80 Lestrade, L. \& Weber, M. J. snoRNA-LBME-db, a comprehensive database of human H/ACA and C/D box snoRNAs. Nucleic Acids Res 34, D158-162, doi:10.1093/nar/gkj002 (2006).

81 Hebras, J., Krogh, N., Marty, V., Nielsen, H. \& Cavaille, J. Developmental changes of rRNA ribose methylations in the mouse. RNA Biol 17, 150-164, doi:10.1080/15476286.2019.1670598 (2020).

82 Cooper, G. M. The Cell: A Molecular Approach. 2nd edn, (Sinauer Associates, 2000).

83 Henras, A. K., Plisson-Chastang, C., O'Donohue, M. F., Chakraborty, A. \& Gleizes, P. E. An overview of pre-ribosomal RNA processing in eukaryotes. Wiley Interdiscip Rev RNA 6, 225-242, doi:10.1002/wrna.1269 (2015).

84 Erales, J. et al. Evidence for rRNA 2'-O-methylation plasticity: Control of intrinsic translational capabilities of human ribosomes. Proc Natl Acad Sci U S A 114, 1293412939, doi:10.1073/pnas.1707674114 (2017).

85 Penzo, M. et al. Human ribosomes from cells with reduced dyskerin levels are intrinsically altered in translation. FASEB J 29, 3472-3482, doi:10.1096/fj.15-270991 (2015). 
Jack, K. et al. rRNA pseudouridylation defects affect ribosomal ligand binding and translational fidelity from yeast to human cells. Mol Cell 44, 660-666, doi:10.1016/j.molcel.2011.09.017 (2011).

87 Skreka, K. et al. Identification of differentially expressed non-coding RNAs in embryonic stem cell neural differentiation. Nucleic Acids Res 40, 6001-6015, doi:10.1093/nar/gks311 (2012).

88 Warner, W. A. et al. Expression profiling of snoRNAs in normal hematopoiesis and AML. Blood Adv 2, 151-163, doi:10.1182/bloodadvances.2017006668 (2018).

89 Skrzypczyk, A. et al. Noncoding RNA Transcripts during Differentiation of Induced Pluripotent Stem Cells into Hepatocytes. Stem Cells Int 2018, 5692840, doi:10.1155/2018/5692840 (2018).

90 Higa-Nakamine, S. et al. Loss of ribosomal RNA modification causes developmental defects in zebrafish. Nucleic Acids Res 40, 391-398, doi:10.1093/nar/gkr700 (2012).

91 Velichutina, I. V. et al. Mutations in helix 27 of the yeast Saccharomyces cerevisiae $18 \mathrm{~S}$ rRNA affect the function of the decoding center of the ribosome. RNA 6, 11741184 (2000).

92 Sergiev, P. V., Bogdanov, A. A. \& Dontsova, O. A. Ribosomal RNA guanine-(N2)methyltransferases and their targets. Nucleic Acids Res 35, 2295-2301, doi:10.1093/nar/gkm104 (2007).

93 Liiv, A., Karitkina, D., Maivali, U. \& Remme, J. Analysis of the function of E. coli $23 \mathrm{~S}$ rRNA helix-loop 69 by mutagenesis. BMC Mol Biol 6, 18, doi:10.1186/1471-2199-6-18 (2005).

94 Greber, B. J. et al. The complete structure of the large subunit of the mammalian mitochondrial ribosome. Nature 515, 283-286, doi:10.1038/nature13895 (2014).

95 Marchand, V., Blanloeil-Oillo, F., Helm, M. \& Motorin, Y. Illumina-based RiboMethSeq approach for mapping of 2'-O-Me residues in RNA. Nucleic Acids Res 44, e135, doi:10.1093/nar/gkw547 (2016).

96 Lovejoy, A. F., Riordan, D. P. \& Brown, P. O. Transcriptome-wide mapping of pseudouridines: pseudouridine synthases modify specific mRNAs in S. cerevisiae. PLoS One 9, e110799, doi:10.1371/journal.pone.0110799 (2014).

97 Goldring, M. B. \& Marcu, K. B. Cartilage homeostasis in health and rheumatic diseases. Arthritis Res Ther 11, 224, doi:10.1186/ar2592 (2009).

98 Troeberg, L. \& Nagase, H. Proteases involved in cartilage matrix degradation in osteoarthritis. Biochim Biophys Acta 1824, 133-145, doi:10.1016/j.bbapap.2011.06.020 (2012).

99 Loeser, R. F., Goldring, S. R., Scanzello, C. R. \& Goldring, M. B. Osteoarthritis: a disease of the joint as an organ. Arthritis Rheum 64, 1697-1707, doi:10.1002/art.34453 (2012).

100 Ripmeester, E. G. J., Timur, U. T., Caron, M. M. J. \& Welting, T. J. M. Recent Insights into the Contribution of the Changing Hypertrophic Chondrocyte Phenotype in the 
Development and Progression of Osteoarthritis. Front Bioeng Biotechnol 6, 18, doi:10.3389/fbioe.2018.00018 (2018).

101 Atsumi, T., Miwa, Y., Kimata, K. \& Ikawa, Y. A chondrogenic cell line derived from a differentiating culture of AT805 teratocarcinoma cells. Cell Differ Dev 30, 109-116 (1990).

102 Le, L. T., Swingler, T. E. \& Clark, I. M. Review: the role of microRNAs in osteoarthritis and chondrogenesis. Arthritis Rheum 65, 1963-1974, doi:10.1002/art.37990 (2013).

103 Langhendries, J. L., Nicolas, E., Doumont, G., Goldman, S. \& Lafontaine, D. L. The human box C/D snoRNAs U3 and U8 are required for pre-rRNA processing and tumorigenesis. Oncotarget 7, 59519-59534, doi:10.18632/oncotarget.11148 (2016).

104 Peffers, M., Liu, X. \& Clegg, P. Transcriptomic signatures in cartilage ageing. Arthritis Res Ther 15, R98, doi:10.1186/ar4278 (2013).

105 Peffers, M. J. et al. Transcriptome analysis of ageing in uninjured human Achilles tendon. Arthritis Res Ther 17, 33, doi:10.1186/s13075-015-0544-2 (2015).

106 Zhao, Y. et al. Expression signature of six-snoRNA serves as novel non-invasive biomarker for diagnosis and prognosis prediction of renal clear cell carcinoma. J Cell Mol Med 24, 2215-2228, doi:10.1111/jcmm.14886 (2020).

107 Steinbusch, M. M. et al. Serum snoRNAs as biomarkers for joint ageing and post traumatic osteoarthritis. Sci Rep 7, 43558, doi:10.1038/srep43558 (2017).

108 Poulet, B. Models to define the stages of articular cartilage degradation in osteoarthritis development. Int J Exp Pathol 98, 120-126, doi:10.1111/iep.12230 (2017).

109 Appleton, C. T., Pitelka, V., Henry, J. \& Beier, F. Global analyses of gene expression in early experimental osteoarthritis. Arthritis Rheum 56, 1854-1868, doi:10.1002/art.22711 (2007).

110 Wei, T. et al. Analysis of early changes in the articular cartilage transcriptisome in the rat meniscal tear model of osteoarthritis: pathway comparisons with the rat anterior cruciate transection model and with human osteoarthritic cartilage. Osteoarthritis Cartilage 18, 992-1000, doi:10.1016/j.joca.2010.04.012 (2010).

111 Loeser, R. F. et al. Microarray analysis reveals age-related differences in gene expression during the development of osteoarthritis in mice. Arthritis Rheum 64, 705-717, doi:10.1002/art.33388 (2012).

112 Turnbull, I. R. et al. Effects of age on mortality and antibiotic efficacy in cecal ligation and puncture. Shock 19, 310-313, doi:10.1097/00024382-200304000-00003 (2003).

113 Glasson, S. S., Blanchet, T. J. \& Morris, E. A. The surgical destabilization of the medial meniscus (DMM) model of osteoarthritis in the 129/SvEv mouse. Osteoarthritis Cartilage 15, 1061-1069, doi:10.1016/j.joca.2007.03.006 (2007).

114 Zhang, L. et al. Serum non-coding RNAs as biomarkers for osteoarthritis progression after ACL injury. Osteoarthritis Cartilage 20, 1631-1637, doi:10.1016/j.joca.2012.08.016 (2012). 
115 Okugawa, Y. et al. Clinical significance of SNORA42 as an oncogene and a prognostic biomarker in colorectal cancer. Gut 66, 107-117, doi:10.1136/gutjnl-2015-309359 (2017).

116 Liao, J. et al. Small nucleolar RNA signatures as biomarkers for non-small-cell lung cancer. Mol Cancer 9, 198, doi:10.1186/1476-4598-9-198 (2010).

117 Kishikawa, T. et al. Circulating RNAs as new biomarkers for detecting pancreatic cancer. World J Gastroenterol 21, 8527-8540, doi:10.3748/wjg.v21.i28.8527 (2015).

118 Hunter, M. P. et al. Detection of microRNA expression in human peripheral blood microvesicles. PLoS One 3, e3694, doi:10.1371/journal.pone.0003694 (2008).

119 Enderle, D. et al. Characterization of RNA from Exosomes and Other Extracellular Vesicles Isolated by a Novel Spin Column-Based Method. PLoS One 10, e0136133, doi:10.1371/journal.pone.0136133 (2015).

120 Kuyinu, E. L., Narayanan, G., Nair, L. S. \& Laurencin, C. T. Animal models of osteoarthritis: classification, update, and measurement of outcomes. J Orthop Surg Res 11, 19, doi:10.1186/s13018-016-0346-5 (2016).

121 McCoy, A. M. Animal Models of Osteoarthritis: Comparisons and Key Considerations. Vet Pathol 52, 803-818, doi:10.1177/0300985815588611 (2015).

122 Peffers, M. J., Clegg, P. D., Boothroyd, E., Cremers, A., Surtel, D. A., Caron, M. M., Welting, T. J. BIG tasks for small RNAs; a new class of RNAs in the pathogenesis of osteoarthritis. Osteoarthritis and Cartilage 24 (2016).

123 Duker, A. L. et al. Paternally inherited microdeletion at $15 q 11.2$ confirms a significant role for the SNORD116 C/D box snoRNA cluster in Prader-Willi syndrome. Eur J Hum Genet 18, 1196-1201, doi:10.1038/ejhg.2010.102 (2010).

124 Holm, V. A. et al. Prader-Willi syndrome: consensus diagnostic criteria. Pediatrics 91, 398-402 (1993).

125 Butler, M. G. et al. Decreased bone mineral density in Prader-Willi syndrome: comparison with obese subjects. Am J Med Genet 103, 216-222 (2001).

126 Khor, E. C. et al. Prader-Willi Critical Region, a Non-Translated, Imprinted Central Regulator of Bone Mass: Possible Role in Skeletal Abnormalities in Prader-Willi Syndrome. PLoS One 11, e0148155, doi:10.1371/journal.pone.0148155 (2016).

127 Karlsson, M. K., Magnusson, H., Coster, M., Karlsson, C. \& Rosengren, B. E. Patients with knee osteoarthritis have a phenotype with higher bone mass, higher fat mass, and lower lean body mass. Clin Orthop Relat Res 473, 258-264, doi:10.1007/s11999014-3973-3 (2015).

128 Makitie, O. \& Kostjukovits, S. in GeneReviews(R) (eds R. A. Pagon et al.) (1993).

$129 \mathrm{Su}, \mathrm{N} ., \mathrm{Jin}, \mathrm{M}$. \& Chen, L. Role of FGF/FGFR signaling in skeletal development and homeostasis: learning from mouse models. Bone Res 2, 14003, doi:10.1038/boneres.2014.3 (2014).

130 Kiss, T. Small nucleolar RNAs: an abundant group of noncoding RNAs with diverse cellular functions. Cell 109, 145-148 (2002). 
131 Reichow, S. L., Hamma, T., Ferre-D'Amare, A. R. \& Varani, G. The structure and function of small nucleolar ribonucleoproteins. Nucleic Acids Res 35, 1452-1464, doi:10.1093/nar/gkl1172 (2007). 



\section{Chapter 8}

Valorization 
Chapter 8 


\section{Valorization}

\section{snoRNA biomarkers in osteoarthritis and ageing}

Cartilage is an important structural component of the body; essential during walking, sports and everyday life functioning. Due to the lack of vascularization, cartilage is largely hampered in its reparative capacity ${ }^{1}$. This has major implications for cartilage diseases such as osteoarthritis $(\mathrm{OA})$, the most prevalent age-related degenerative joint disease ${ }^{2,3}$. OA affects over 300 million people globally ${ }^{4}$ and is a common cause of chronic disability worldwide ${ }^{2}$. In addition it is a significant contributor to both individual and socioeconomic burden and the number of disability adapted life years globally ${ }^{5}$. If the deterioration in musculoskeletal health and development of OA can be identified and treated early, serious life impairment may be abrogated. The development of effective treatments for OA and the ability to predict disease progression has been hampered by the lack of biomarkers able to demonstrate pathological disturbances preceding identifiable tissue alterations. Chapter $\mathbf{5}$ of this thesis has contributed to expanding this knowledge by the identification of snoRNAbased biomarkers that are indicative for ageing and/or for OA. For example, SNORA73 was increased in old joint and serum and thus represents a potential joint 'biological ageing' marker ${ }^{6}$. SNORA64 was increased and SNORD46 was decreased in serum in a mouse OA model, where OA was established by destabilization of the medial meniscus (DMM), but both were not differentially expressed in young versus old serum, highlighting these snoRNAs as possible OA markers ${ }^{6}$. SNORD18 was increased in serum both in ageing and following DMM, suggesting that it is affected in both ageing and OA. The most differentially expressed snoRNA in mouse DMM serum was SNORD116 ${ }^{6}$. This increase was confirmed in serum of horses with metacarpophalangeal (MCP; joint with similarities to the human knee joint $^{7}$ ) OA. Additionally, SNORD116 has previously been identified as increased in OA compared to normal human cartilage in a micro-array study ${ }^{8}$, indicating that SNORD116 function might be conserved between species. Ideally, biomarkers should be assessable via low-invasive methods and serum is thus a good source for biomarker measurement. The snoRNA biomarkers identified in our work represent a class of bio-molecules for which quantitative assays can be set-up with relative ease. Following our findings it would thus be a possibility that snoRNAs could add to a diagnostic set of serum-based bio-molecules 


\section{Chapter 8}

indicative for biological age and OA status of the patient. Given the fact that we used a mouse model, effort should be put in the validation of our findings for the human situation, as well as the set-up of large cohort studies, where $O A$ is well-defined and a relation between the disease stage and the specific snoRNA in the serum may be deduced. In addition, the snoRNAs' specificity should be investigated further to determine whether the situation in the joint is represented in the serum and for example how to extrapolate the results in case of co-morbidities.

\section{snoRNAs in chondrogenic development and Cartilage Hair Hypoplasia}

Mutations in the RMRP gene are the cause of a severe form of dwarfism known as cartilagehair hypoplasia ${ }^{9}\left(\mathrm{CHH}\right.$, McKusick-type metaphyseal chondrodysplasia $\left.{ }^{10}\right)$, which is part of the anauxetic dysplasia spectrum of disorders ${ }^{11,12}$. To date, more than hundred individual $\mathrm{CHH}$ pathogenic mutations have been identified in the RMRP gene ${ }^{9}$. Although relatively rare in the general population, the disease prevalence is exceptionally high among the Amish and Finnish populations ${ }^{13}$. Moreover, the disease consequences for those identified with the disease can be considered severe; one predominant phenotypic hallmark of $\mathrm{CHH}$ is shortlimbed dwarfism caused by abnormal growth plate development. Other symptoms include sparse thin hair, anaemia, Hirschsprung's disease, bronchiectasis, and impaired T-cell immunity. In addition, adult patients have a predisposition to certain cancers (i.e. squamous cell carcinoma, basal cell carcinoma and non-Hodgkin lymphoma) $)^{12,14}$. The data and insight generated through investigation of this human disease model may provide further insight in the role of RMRP RNA, RNase MRP functions and rRNA processing in chondrocyte cell development and function (Chapter 2). Our data showed that RMRP RNA expression is regulated during different stages of chondrogenic differentiation and indicate that $R M R P$ RNA plays a pivotal role in chondrocyte hypertrophy, with consequences for $\mathrm{CHH}$ pathobiology ${ }^{15}$. Interestingly, the gene expression alterations observed in OA cartilage also indicate a replay of chondrogenic differentiation towards chondrocyte hypertrophy ${ }^{16}$. This holds promise that by investigating this rare disease model we could additionally generate findings that contribute to the understanding of the complex etiology of a highly prevalent disease such as OA. However, for $\mathrm{CHH}$ patients, a multi-causal approach will most-probably be required when selecting novel therapeutic targets. Considering the many functions RMRP 
RNA and the RNase MRP complex are involved in, a one-fit-for-all therapy should not be expected, but rather a personalized therapy based on the molecular effects of the diverse RMRP mutations and the overall disease phenotype. Treatment of $\mathrm{CHH}$ with growth hormone to support skeletal growth has been reported ${ }^{17}$, but efficacy and safety are under debate. Recently CRISPR genome editing is being put forward for treatment of cystic fibrosis $^{18}$. Considering the isolated genomic nature of RMRP mutations leading to $\mathrm{CHH}$, this disease might represent a good candidate for CRISPR genome editing, and recent advancements in CRISPR genome editing may shed light on the future treatment options for $\mathrm{CHH}$. However, the tissue delivery of such genome editing tools are expected to be challenging in $\mathrm{CHH}$ and in particular in the case of targeting developing cartilage. In relation to this notion it will therefore be important to additionally investigate the deregulated molecular networks downstream of RMRP in tissues with a high $\mathrm{CHH}$-burden. This will potentially allow counteracting of the pathological molecular aberrations caused by mutations in RMRP in a downstream (symptomatic) manner. Our finding that viperin regulates a CXCL10-TGF- $\beta / S M A D 2 / 3$ axis during chondrogenic differentiation, which is deregulated in $\mathrm{CHH}$ due to abnormal expression of viperin (Chapter 3$)^{19}$, might provide a starting point for such an approach. For example by targeting factors downstream of viperin, such as CXCL10 and investigating the possibility of an interferon-related therapy. In Chapter 4, snoRNAs were found to be differentially expressed during ATDC5 chondrogenic differentiation, with impact on the translational capacity of the cell. Even though here we have not yet identified isolated snoRNAs and their specific function, they appear to be vital for the overall outcome of chondrogenesis. Understanding and influencing chondrogenesis will be key for cartilage regenerative therapies (e.g. cartilage tissue engineering using MSCs) in the context of, for example, OA. Our data indicate that snoRNAs might be good candidates to target and influence the course of chondrogenesis. In this respect antisense oligonucleotides (ASOs) may proof a valuable means in targeting these snoRNAs in the future ${ }^{20}$. A wide range of modifications to ASOs have already been investigated to improve their stability, influence their mechanism of action or steer their delivery ${ }^{20}$. ASOs have been investigated in preclinical studies such as in vitro and small animal in vivo studies and several formulations were already regulatory (FDA / EMA) approved for use in the $\operatorname{clinic}^{20-23}$. Our group has recently been successfully using second generation ASOs for targeting snoRNA expression levels in chondrocytes ${ }^{24,25}$. These ASOs are designed in a 5-10-5 gapmer 
Chapter 8

configuration and have a phosphorotioate backbone, containing a core of 10 DNA nucleotides, flanked on both sides by modified (2'O-ribose methyl) RNA nucleotides. These ASOs are RNase $\mathrm{H} 1$ dependent and effective in targeting snoRNAs ${ }^{26}$. Therefore, using ASOs to target snoRNAs in the context of chondrogenic development would be a promising perspective for future $O A$ therapies as well. However, before we can enter this stage, functional studies will be needed to investigate the function of specific snoRNAs in the context of chondrogenesis to select promising snoRNA targets. 


\section{References}

1 Mandal, A. What is Cartilage? News Medical Life Sciences, <https://www.newsmedical.net/health/What-is-Cartilage.aspx> (2017).

2 Woolf, A. D. \& Pfleger, B. Burden of major musculoskeletal conditions. Bull World Health Organ 81, 646-656 (2003).

3 Li, Y., Wei, X., Zhou, J. \& Wei, L. The age-related changes in cartilage and osteoarthritis. Biomed Res Int 2013, 916530, doi:10.1155/2013/916530 (2013).

4

Disease, G. B. D., Injury, I. \& Prevalence, C. Global, regional, and national incidence, prevalence, and years lived with disability for 354 diseases and injuries for 195 countries and territories, 1990-2017: a systematic analysis for the Global Burden of Disease Study 2017. Lancet 392, 1789-1858, doi:10.1016/S0140-6736(18)32279-7 (2018).

5 Hunter, D. J., Schofield, D. \& Callander, E. The individual and socioeconomic impact of osteoarthritis. Nat Rev Rheumatol 10, 437-441, doi:10.1038/nrrheum.2014.44 (2014).

6 Steinbusch, M. M. et al. Serum snoRNAs as biomarkers for joint ageing and post traumatic osteoarthritis. Sci Rep 7, 43558, doi:10.1038/srep43558 (2017).

7 McCoy, A. M. Animal Models of Osteoarthritis: Comparisons and Key Considerations. Vet Pathol 52, 803-818, doi:10.1177/0300985815588611 (2015).

8 Peffers, M. J. et al. BIG tasks for small RNAs; a new class of rnas in the pathgenesis of osteoarthritis. Osteoarthritis and Cartilage 24, S372 (2016).

9 Mattijssen, S., Welting, T. J. \& Pruijn, G. J. RNase MRP and disease. Wiley Interdiscip Rev RNA 1, 102-116, doi:10.1002/wrna.9 (2010).

10 Ridanpaa, M. et al. Mutations in the RNA component of RNase MRP cause a pleiotropic human disease, cartilage-hair hypoplasia. Cell 104, 195-203 (2001).

11 Thiel, C. T. et al. Severely incapacitating mutations in patients with extreme short stature identify RNA-processing endoribonuclease RMRP as an essential cell growth regulator. Am J Hum Genet 77, 795-806, doi:10.1086/497708 (2005).

12 Makitie, O. \& Kostjukovits, S. in GeneReviews(R) (eds R. A. Pagon et al.) (1993).

13 Makitie, O. Cartilage-hair hypoplasia in Finland: epidemiological and genetic aspects of 107 patients. J Med Genet 29, 652-655, doi:10.1136/jmg.29.9.652 (1992).

14 Shiasi Arani, K. Clinical features and management of Cartilage-Hair Hypoplasia: a narrative review. J Pediatr Rev 3(1):e194, 8, doi:10.5812/jpr.194 (2015).

15 Steinbusch, M. M. F. et al. Expression of RMRP RNA is regulated in chondrocyte hypertrophy and determines chondrogenic differentiation. Sci Rep 7, 6440, doi:10.1038/s41598-017-06809-5 (2017).

16 Ripmeester, E. G. J., Timur, U. T., Caron, M. M. J. \& Welting, T. J. M. Recent Insights into the Contribution of the Changing Hypertrophic Chondrocyte Phenotype in the Development and Progression of Osteoarthritis. Front Bioeng Biotechnol 6, 18, doi:10.3389/fbioe.2018.00018 (2018).

17 Obara-Moszynska, M., Wielanowska, W., Rojek, A., Wolnik-Brzozowska, D. \& Niedziela, M. Treatment of cartilage-hair hypoplasia with recombinant human growth hormone. Pediatr Int 55, e162-164, doi:10.1111/ped.12215 (2013).

18 Marangi, M. \& Pistritto, G. Innovative Therapeutic Strategies for Cystic Fibrosis: Moving Forward to CRISPR Technique. Front Pharmacol 9, 396, doi:10.3389/fphar.2018.00396 (2018). 
19 Steinbusch, M. M. F. et al. The antiviral protein viperin regulates chondrogenic differentiation via CXCL10 protein secretion. J Biol Chem 294, 5121-5136, doi:10.1074/jbc.RA119.007356 (2019).

20 Benizri, S. et al. Bioconjugated Oligonucleotides: Recent Developments and Therapeutic Applications. Bioconjug Chem 30, 366-383, doi:10.1021/acs.bioconjchem.8b00761 (2019).

21 Schoch, K. M. \& Miller, T. M. Antisense Oligonucleotides: Translation from Mouse Models to Human Neurodegenerative Diseases. Neuron 94, 1056-1070, doi:10.1016/j.neuron.2017.04.010 (2017).

22 Evers, M. M., Toonen, L. J. \& van Roon-Mom, W. M. Antisense oligonucleotides in therapy for neurodegenerative disorders. Adv Drug Deliv Rev 87, 90-103, doi:10.1016/j.addr.2015.03.008 (2015).

23 Herrera, V. L., Colby, A. H., Ruiz-Opazo, N., Coleman, D. G. \& Grinstaff, M. W. Nucleic acid nanomedicines in Phase II/III clinical trials: translation of nucleic acid therapies for reprogramming cells. Nanomedicine (Lond) 13, 2083-2098, doi:10.2217/nnm2018-0122 (2018).

24 Peffers, M. J. et al. SnoRNA signatures in cartilage ageing and osteoarthritis. Sci Rep 10, 10641, doi:10.1038/s41598-020-67446-z (2020).

25 Ripmeester, E. G., Caron, M.M., Balaskas, P., Dyer, P., Chabronova, A., van den Akker, G.G., Housmans, B.A., Smagul, A., Fang, Y., Cremers, A., Surtel, D.A., van Rhijn, L.W., Peffers, M.J., Welting, T.J. Impaired chondrocyte U3 snoRNA expression in osteoarthritis impacts the chondrocyte protein translation apparatus. Scientific Reports, accepted for publication (2020).

26 Liang, X. H., Sun, H., Nichols, J. G. \& Crooke, S. T. RNase H1-Dependent Antisense Oligonucleotides Are Robustly Active in Directing RNA Cleavage in Both the Cytoplasm and the Nucleus. Mol Ther 25, 2075-2092, doi:10.1016/j.ymthe.2017.06.002 (2017). 
Valorization 



\section{Chapter 9}

Summary 
Chapter 9 


\section{Summary}

The cellular processes in which snoRNAs participate are fundamental processes needed for proper cell function, yet their roles in cell differentiation, homeostasis and disease in general have been poorly investigated. Moreover, in the context of chondrogenic differentiation, cartilage homeostasis and disease, snoRNAs have not been investigated so far. In the work presented in this thesis we therefore investigated the involvement of snoRNAs in several chondrocyte and cartilage facets.

\section{Chapter 2 Expression of RMRP RNA is regulated in chondrocyte hypertrophy and determines chondrogenic differentiation}

Mutations in the RMRP-gene, encoding the snoRNA component of the RNase MRP complex, are the cause of cartilage-hair hypoplasia $(\mathrm{CHH})^{1,2}$. $\mathrm{CHH}$ is associated with severe dwarfism caused by impaired skeletal development. However, it is not clear why mutations in $R M R P$ snoRNA lead to skeletal dysplasia. Since chondrogenic differentiation of the growth plate is required for development of the long bones, we hypothesized that the RMRP snoRNA plays a pivotal role in chondrogenic differentiation. Expression of Rmrp RNA and RNase MRP protein subunits was detected in the murine growth plate and during the course of chondrogenic differentiation of ATDC5 cells, where Rmrp RNA expression was found to be correlated with chondrocyte hypertrophy. Genetic interference with Rmrp RNA expression in ATDC5 cells caused a deregulation of chondrogenic differentiation, with a prominent impact on hypertrophy and changes in pre-rRNA processing and rRNA levels (reduced levels of $18 \mathrm{~S}$ and 5.8S rRNA). Promoter reporter studies showed that Rmrp RNA expression responds to chondrogenic morphogens such as PTHrP and bFGF (reduced promoter activity) and TGF $\beta 3$, BMP-2, WNT-3A and WNT-5A (increased promoter activity). Chondrogenic transdifferentiation of $\mathrm{CHH}$ fibroblasts was impaired with a pronounced impact on hypertrophic differentiation, increased levels of PTHrP and accumulation of the ITS-1 pre-rRNA processing intermediate. Together, our data show that RMRP RNA expression is regulated during different stages of chondrogenic differentiation and indicate that RMRP RNA may play a pivotal role in chondrocyte hypertrophy, with potential consequences for $\mathrm{CHH}$ pathobiology ${ }^{3}$. 


\section{Chapter 3 The anti-viral protein viperin regulates chondrogenic differentiation via CXCL10 protein secretion}

RNase MRP has a number of substrate RNAs and from most it is not clear how these substrate RNAs may influence cell biological processes, nor is it known how these substrates may be involved in the development of $\mathrm{CHH}$ or chondrogenic differentiation. One of these substrate RNAs is the mRNA of viperin ${ }^{4}$. Viperin, an abbreviation for Virus Inhibitory Protein, Endoplasmic Reticulum-associated, Interferon (IFN)-inducible, is a protein located in the endoplasmic reticulum ${ }^{5}$ and it is well described for its role as an antiviral protein ${ }^{6}$. Viperin expression has been shown to be increased in $\mathrm{CHH}$ leukocytes ${ }^{7}$ and following knockdown of RNase MRP subunits ${ }^{4}$. We discovered that viperin is expressed in differentiating chondrocytic cells and regulates their protein secretion and the outcome of chondrogenic differentiation by influencing TGF- $\beta /$ SMAD2/3 activity via CXCL10, where CXCL10 inhibits chondrogenic differentiation. Moreover, we observed disturbances in this viperin-CXCL10TGF- $\beta / S M A D 2 / 3$ axis in $\mathrm{CHH}$ chondrocytic cells. Our results indicate that the anti-viral protein viperin controls chondrogenic differentiation by influencing secretion of soluble proteins and we identified a molecular route that may explain impaired chondrogenic differentiation of cells from individuals with $\mathrm{CHH}^{8}$.

\section{Chapter 4 Adaptation of protein translational apparatus during ATDC5 chondrogenic differentiation}

The RMRP snoRNA is one highly specific snoRNA belonging to a small group of non-canonical snoRNAs. The majority of the cell's snoRNAs however belong to a group of canonical snoRNAs involved in the post-transcriptional modification of rRNAs. Fine-tuning of the cell's rRNA pool by snoRNA-mediated post-transcriptional modifications is believed to determine ribosome activity and control ribosome translation fidelity. It is expected that this is particularly important in translationally active cells, like growth plate chondrocytes, in order to accurately and efficiently synthesize the proteins required for building the cartilaginous growth plate extracellular matrix and support their high speed of proliferation. We therefore charted the full spectrum of snoRNAs expressed during different phases of chondrogenic differentiation. snoRNAs were found to be differentially expressed during ATDC5 
chondrogenic differentiation. In addition, rRNA post-transcriptional modifiers, the 2'O-ribose methylase fibrillarin and the pseudouridylase dyskerin, as well as UBF-1 (involved in rDNA transcription) expression and 18S, 5.8S and 28S rRNA content per cell adapted to the differentiation status of ATDC5 cells. Overall an impact on the translational capacity of the cell, depending on the differentiation status of the ATDC5 cells, was demonstrated. Thus, our data showed that chondrogenic differentiation is associated with significant regulation of mechanisms involving ribosome biogenesis and translation activity. Differentiation-phase specific expression of snoRNAs suggests that specific snoRNAs may modulate the chondrocyte's developing phenotype via an rRNA post-transcriptional modification-based ribosome heterogeneity mechanism, thereby potentially facilitating the observed dynamics in translational activity impacting the course of chondrogenic differentiation. Future work is expected to uncover the extent of ribosome heterogeneity and regulation in cellular differentiation and its potential implications for human disease.

\section{Chapter 5 Serum snoRNAs as biomarkers for joint ageing and post- traumatic osteoarthritis}

In osteoarthritis, like in chondrogenic differentiation, the chondrogenic phenotype is actively changing ${ }^{9}$. The development of effective treatments for the age-related disease osteoarthritis and the ability to predict disease progression has been hampered by the lack of biomarkers able to demonstrate the course of the disease. Profiling the expression patterns of snoRNAs in murine joint ageing and osteoarthritis may provide insight in their contribution to joint pathology, their use as diagnostic biomarkers and potential as therapeutic targets. SnoRNASeq identified differential expression of 6 snoRNAs in young versus old joints and 5 snoRNAs in old sham versus old experimental osteoarthritic joints. In serum we found differential presence of 27 snoRNAs in young versus old serum and 18 snoRNAs in old sham versus old experimental osteoarthritic serum. Profiling the expression patterns of snoRNAs is the initial step in determining their functional significance in ageing and osteoarthritis, and provides potential diagnostic biomarkers and therapeutic targets. Our results established snoRNAs as novel markers of musculoskeletal ageing and osteoarthritis and implicate specific changes in snoRNA abundance in joint ageing (SNORD88 and SNORD38 were respectively decreased and increased) and OA, suggesting the potential 


\section{Chapter 9}

use of snoRNAs such as SNORA73 and SNORD23 as a novel biomarker for joint ageing, SNORA64, SNORD46 and SNORD116 for OA, SNORD18 for ageing and OA ${ }^{10}$.

\section{Conclusions}

In the general discussion the data described in this thesis were discussed with respect to each other and with outsight to future directions for snoRNA research in cartilaginous tissues. In addition, we provided an overview of the molecular interactions identified from this thesis. Overall, the work presented in this thesis provides directions for pathways in which snoRNAs function in chondrocyte development and disease. 


\section{References}

1 Thiel, C. T. et al. Severely incapacitating mutations in patients with extreme short stature identify RNA-processing endoribonuclease RMRP as an essential cell growth regulator. Am J Hum Genet 77, 795-806, doi:10.1086/497708 (2005).

2 Ridanpaa, M. et al. Mutations in the RNA component of RNase MRP cause a pleiotropic human disease, cartilage-hair hypoplasia. Cell 104, 195-203 (2001).

3 Steinbusch, M. M. F. et al. Expression of RMRP RNA is regulated in chondrocyte hypertrophy and determines chondrogenic differentiation. Sci Rep 7, 6440, doi:10.1038/s41598-017-06809-5 (2017).

4 Mattijssen, S. et al. Viperin mRNA is a novel target for the human RNase MRP/RNase P endoribonuclease. Cell Mol Life Sci 68, 2469-2480, doi:10.1007/s00018-010-0568-3 (2011).

5 Hinson, E. R. \& Cresswell, P. The antiviral protein, viperin, localizes to lipid droplets via its N-terminal amphipathic alpha-helix. Proc Natl Acad Sci U S A 106, 2045220457, doi:10.1073/pnas.0911679106 (2009).

6 Mattijssen, S. \& Pruijn, G. J. Viperin, a key player in the antiviral response. Microbes Infect 14, 419-426, doi:10.1016/j.micinf.2011.11.015 (2012).

7 Hermanns, P. et al. Consequences of mutations in the non-coding RMRP RNA in cartilage-hair hypoplasia. Hum Mol Genet 14, 3723-3740, doi:10.1093/hmg/ddi403 (2005).

8 Steinbusch, M. M. F. et al. The antiviral protein viperin regulates chondrogenic differentiation via CXCL10 protein secretion. J Biol Chem 294, 5121-5136, doi:10.1074/jbc.RA119.007356 (2019).

9 Le, L. T., Swingler, T. E. \& Clark, I. M. Review: the role of microRNAs in osteoarthritis and chondrogenesis. Arthritis Rheum 65, 1963-1974, doi:10.1002/art.37990 (2013).

10 Steinbusch, M. M. et al. Serum snoRNAs as biomarkers for joint ageing and post traumatic osteoarthritis. Sci Rep 7, 43558, doi:10.1038/srep43558 (2017). 



\section{Curriculum Vitae}




\section{Curriculum Vitae}

Mandy Meekels-Steinbusch was born in Brunssum, the Netherlands on November 13, 1987. In 2007 she graduated from pre-university education (Atheneum, Romboutscollege) with a background in Economics and Society and started a Bachelor of Science in Health Sciences at Maastricht University with a major in Bioregulation and a minor in Movement Sciences. From 2010 to 2012 she furthered her education at Maastricht University

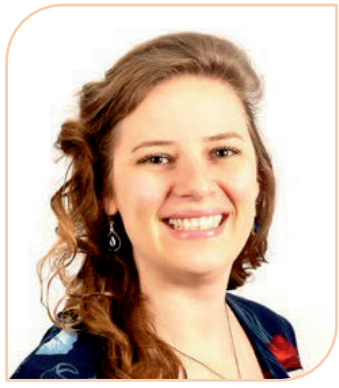
with a Master of Science in Molecular Life Sciences / Research Master in Nutrition and Metabolism; Fundamental and Clinical Aspects (school of Nutrition and Translational Medicine). During her entire university education, Mandy had the opportunity to participate in several research internships. For her major bachelor internship, Mandy investigated the beneficial effects of sitostanol on the attenuated immune function in asthma patients at the department of Human Biology under the supervision of Dr. Brüll and Dr. Plat, the results of which were published in PLOS One, with Mandy as co-author. For her junior master internship, Mandy investigated properdin as the trigger for complement activation in patients with non-alcoholic fatty liver disease at the department of Surgery under the supervision of Dr. Segers and Dr. Rensen. Finally, for her senior master internship, Mandy tied lysosomal cholesterol accumulation to inflammasomes during liver inflammation in nonalcoholic steatohepatitis as observed in hyperlipidemic mice. This senior master internship was performed at the department of Molecular Genetics under the supervision of Dr. Hendrikx and Dr. Shiri-Sverdlov and the results were published in PLOS One (co-authorship). During her education Mandy also won the Top 3\% Certificate thrice, which is a grant that gives the top $3 \%$ of students enrolled in Maastricht University the opportunity to study free of charge by refunding their tuition fees.

From November 2012 to July 2017 Mandy worked as a PhD candidate with her promotores Prof. Dr. Welting and Prof. Dr. van Rhijn at the Laboratory for Experimental Orthopedics / department of Orthopedic Surgery at Maastricht University and the Academic Hospital of Maastricht. The PhD position was part of a bi-lateral cooperation grant from the Netherlands Organization for Scientific Research (NWO) and the Deutsche Forschungsgemeinschaft (DFG, 
German Research Foundation) and required frequent research at the department of Pediatrics in the Medical Center of the University of Freiburg, Germany, led by Dr. Lausch and Prof. Dr. Zabel. In 2016, Mandy performed an additional foreign PhD internship with Prof. Dr. Peffers at the Institute of Ageing and Chronic Disease at the University of Liverpool, UK. With her expertise in conducting research on small nucleolar RNAs (snoRNAs), Mandy set-up the molecular techniques required for the detection of snoRNAs in ageing and osteoarthritis, results of which were published in Nature Scientific Reports as part of this thesis.

Since March 2018, Mandy has made the transition from academia to industry by working at Lonza Netherlands. At first, she started working as a Technology Development and Integration Associate under the supervision of Dr. Sadr and in July 2019 she was promoted to Scientist Bioanalytical Services and Process Development, where she reports to Dr. Champluvier. As a scientist, she is in the lead of and acts as a Subject Matter Expert for the validation and method transfer of advanced therapeutic medicinal products from external clients to Lonza Netherlands' cell and gene therapy manufacturing facility. She is proud to be part of the production, validation and release of the life-saving medicines for the severely ill patients who depend on these therapies. In addition, in 2020, Mandy procured her license as Biosafety Officer via the National Institute for Public Health and the Environment (RIVM / Bureau Genetisch Gemodificeerde Organismen). 

List of publications 


\section{List of publications}

\section{Publications}

Steinbusch MMF, Caron MMJ, Surtel DAM, van den Akker GGH, van Dijk PJ, Friedrich F, Zabel $B$, van Rhijn LW, Peffers MJ, Welting TJM. The anti-viral protein viperin regulates chondrogenic differentiation via CXCL10 protein secretion. Journal of Biological Chemistry 2019. J Biol Chem (2019) 294 (13) 5121-5136, DOI: 10.1074/jbc.RA119.007356

Steinbusch MMF, Caron MMJ, Surtel DAM, Friedrich F, Lausch E, Pruijn GJM, Verhesen W, Schroen BLM, van Rhijn LW, Zabel B, Welting TJM. Expression of RMRP RNA is regulated in chondrocyte hypertrophy and determines chondrogenic differentiation. Nature Scientific Reports 2017. Sci Rep 7 (2017), 6440, DOI: 10.1038/s41598-017-06809-5

Steinbusch MMF, Fang Y, Milner PI, Clegg PD, Young DA, Welting TJM, Peffers MJ. Serum snoRNAs as biomarkers for joint ageing and post traumatic osteoarthritis. Nature Scientific Reports 2017. Sci Rep 7 (2017), 43558, DOI: 10.1038/srep43558

Hendrikx T, Bieghs V, Walenbergh SMA, van Gorp PJ, Verheyen F, Jeurissen ML, Steinbusch MMF, Vaes N, Binder CJ, Koek GH, Stienstra R, Netea MG, Hofker MH, Shiri-Sverdlov R. Macrophage Specific Caspase-1/11 Deficiency Protects against Cholesterol Crystallization and Hepatic Inflammation in Hyperlipidemic Mice. PLOS One 2013. 2013; 8(12): e78792

Brüll F, Mensink RP, Steinbusch MF, Husche C, Lütjohann D, Wesseling GJ, Plat J. Beneficial Effects of Sitostanol on the Attenuated Immune Function in Asthma Patients: Results of an In Vitro Approach. PLOS One 2012. 2012; 7(10) e46895: 1-10

\section{Published abstracts}

Steinbusch MMF, Caron MMJ, Surtel DAM, van Dijk PJ, Friedrich F, Zabel B, van Rhijn LW, Peffers MJ, Welting TJM. VIPERIN regulates chondrogenic differentiation via CXCL10 protein secretion. Osteoarthritis and Cartilage 2018 and The University of Liverpool Repository 2018. 26 (2018) S60-S474 and http://livrepository.liverpool.ac.uk/id/eprint/3021985

Peffers MJ, Ripmeester EGJ, Caron MMJ, Steinbusch MMF, Balaskas P, Cremers A, Surtel DAM, Welting TJM. A role for the snoRNA U3 in the altered translational capacity of ageing and osteoarthritic chrondocytes. Osteoarthritis and Cartilage 2018.

DOI: 10.1016/j.joca.2018.02.106 
Steinbusch MMF, Cremers A, van Rhijn LW, Welting TJM. Differential expression of snoRNAs in chondrogenic differentiation. Orthopaedic Proceedings by The British Editorial Society of Bone \& Joint Surgery 2017. 2017, vol. 100-B, suppl. 3. DOI: 10.1302/1358-992X.2018.3.029

Steinbusch MMF, Cremers A, van Rhijn LW, Welting TJM. Unraveling the role of snoRNAs in chondrogenic differentiation. Osteoarthritis and Cartilage 2016.

DOI: 10.2016/j.joca.2016.01.096

Steinbusch MMF, Caron MMJ, Eckmann F, Lausch E, van Rhijn LW, Zabel B, Welting TJM. Viperin; a novel chondrogenic regulator. Osteoarthritis and Cartilage 2015.

DOI: 10.1016/j.joca.2015.02.895

Steinbusch MMF, Caron MMJ, Eckmann F, Lausch E, van Rhijn LW, Zabel B, Welting TJM. Transcriptional regulation of the non-coding snoRNA RMRP during chondrogenic differentiation; a role for NFKB/p65? Osteoarthritis and Cartilage 2014.

DOI: 10.1016/j.joca.2014.02.301

Caron MMJ, Steinbusch MMF, Reichterer K, Matthijssen S, Surtel DAM, van Rhijn LW, Pruijn GJM, Lausch E, Zabel B, Welting TJM. RNase MRP is a novel regulator of endochondral ossification. Osteoarthritis and Cartilage 2013. DOI: 10.1016/j/joca.2013.02.048

Walenbergh S, Hendrikx T, Bieghs V, van Gorp P, Steinbusch MMF, Verheyen F, Rensen S, Buurman W, Olde Damink SWM, Greve JW, Masclee A, Koek G, Hofker M, Shiri-Sverdlov R. The inflammasome as a novel target for the detection and treatment of non-alcoholic steatohepatitis. American Association for the Study of Liver Diseases (AASLD). Hepatology 2012. 56, p.874A-874A $1 p$

\section{Acknowledgements}

Steinbusch MMF stated in acknowledgements for critical reading of the manuscript: Caron MMJ, Emans PJ, Surtel DAM, van der Kraan PM, van Rhijn LW, Welting TJM. BAPX1/NKX-3.2 acts as a chondrocyte hypertrophy molecular switch in osteoarthritis. Arthritis and Rheumatology 2015. 67(11):2944-56. DOI: 10.1002/art.39293

\section{Manuscripts in preparation}

Steinbusch MMF, van den Akker GGH, Cremers A, Caron MMJ, van Rhijn LW, Welting TJM. 2020. Adaptation of the protein translational apparatus during ATDC5 chondrogenic differentiation. Thesis chapter Small nucleolar RNAs in chondrogenic differentiation and osteoarthritis 

List of presentations 


\section{List of presentations}

\section{Conferences with oral presentations}

Ripmeester EGJ, Caron MMJ, Steinbusch MMF, Balaskas P, Chabronova A, van den Akker GGH, Housmans BAC, Cremers A, Surtel DAM, Peffers MJ, Welting TJM. The snoRNA U3 influences the cellular phenotype of chondrocytes and plays a role in their translational capacity. Annual meeting Dutch Society for Matrix Biology (NVMB), 2019

Ripmeester EGJ, Caron MMJ, Steinbusch MMF, Balaskas $\mathrm{P}$, Dyer $\mathrm{P}$, Chabronova $\mathrm{A}$, van den Akker GGH, Housmans BAC, Cremers A, Surtel DAM, Peffers MJ, Welting TJM. The snoRNA U3 influences the cellular phenotype of chondrocytes and plays a role in their translational capacity. European Orthopaedic Research Society (EORS), Maastricht, Netherlands, October 02 $-05,2019$

Peffers MJ, Ripmeester EGJ, Caron MMJ, Steinbusch MMF, Balaskas P, Cremers A, Surtel DAM, Welting TJM. A role for the snoRNA U3 in the altered translational capacity of ageing and osteoarthritic chrondocytes. Osteoarthritis Research Society International (OARSI) world congress on osteoarthritis, Liverpool, UK, April $26-29,2018$

Steinbusch MMF, Cremers A, van Rhijn LW, Welting TJM. Differential expression of snoRNAs in chondrogenic differentiation. European Orthopaedic Research Society (EORS), Munich, Germany, September $13-15,2017$

Steinbusch MMF, Cremers A, Caron MMJ, van Rhijn LW, Peffers MJ, Welting TJM. Differential expression of snoRNAs in chondrogenic differentiation. Osteoarthritis Research Society International (OARSI) world congress on osteoarthritis, Amsterdam, the Netherlands, March 31 - April 03, 2016

Steinbusch MMF, Caron MMJ, Eckmann F, Lausch E, van Rhijn LW, Zabel B, Welting TJM. The RMRP snoRNA and chondrogenic differentiation. Genetics Retreat; the annual Rolduc meeting in the field of human and translational genetics research, Kerkrade, the Netherlands, April 22 24, 2015

Steinbusch MMF substitute oral presenter. Caron MMJ, Emans PJ, Surtel DAM, van der Kraan PM, van Rhijn LW, Welting TJM. BAPX1/NKX3.2; a novel chondrocyte hypertrophy molecular switch in osteoarthritis. Orthopeadic Research Society (ORS) annual meeting, Las Vegas, NV, USA, March 28-April 01, 2015

Steinbusch MMF, Caron MMJ, Eckmann F, Lausch E, van Rhijn LW, Zabel B, Welting TJM. Viperin; a novel chondrogenic regulator. Annual meeting Netherlands Society for Biomaterials 
and Tissue Engineering (NBTE), Lunteren, the Netherlands, December 04 - 05, 2014. Received 'Best Oral Presentation Award of the 2014 annual conference'. Monetary award: $€$ 100,-

Steinbusch MMF, Caron MMJ, Eckmann F, Lausch, E, Zabel B, Pruijn GJM, van Rhijn LW, Welting TJM. RNase MRP is involved in chondrogenic differentiation. $22^{\text {nd }}$ annual meeting Netherlands Society for Biomaterials and Tissue Engineering (NBTE), Lunteren, the Netherlands, November 28 - 29, 2013

Caron MMJ, Steinbusch MMF (presenter), Reichterer K, Matthijssen S, Surtel DAM, Lausch E, Zabel B, Pruijn GJM, van Rhijn LW, Welting TJM. RNase MRP is regulated during skeletal development. $18^{\text {th }}$ annual Osteoarthritis Research Society International (OARSI) world congress on osteoarthritis, Philadelphia, PA, USA, April $18-21,2013$

\section{Conferences with poster presentations}

Steinbusch MMF, Caron MMJ, Surtel DAM, van Dijk PJ, Friedrich F, Zabel B, van Rhijn LW, Peffers MJ, Welting TJM. VIPERIN regulates chondrogenic differentiation via CXCL10 protein secretion. Osteoarthritis Research Society International (OARSI) world congress on osteoarthritis, Liverpool, UK, April 26 - 29, 2018

Steinbusch MMF, Peffers MJ, Cremers A, van Rhijn LW, Welting TJM. Differential expression of snoRNAs in chondrogenic differentiation. RNA: the $21^{\text {th }}$ annual meeting of the RNA society, Kyoto, Japan, June 28 - July 02, 2016.

Peffers MJ, Steinbusch MMF, Milner PI, Fang, Y, Clegg PD, Welting TJM. Identification and evaluation of small nucleolar RNAs as biomarkers for cartilage ageing and osteoarthritis. RNA: the $21^{\text {th }}$ annual meeting of the RNA society, Kyoto, Japan, June 28 - July 02, 2016

Steinbusch MMF, Milner PI, Fang Y, Young DA, Welting TJM, Clegg PD, Peffers MJ. Identification and evaluation of serum snoRNAs as biomarkers for musculoskeletal ageing and osteoarthritis. British Society for Matrix Biology (BSMS) Spring Meeting; the grey area - age and the extracellular matrix, Chester, UK, April 04 - 05, 2016

Friedrich F, Steinbusch MMF, Velasco A, Zabel B, Lausch E, Welting TJM. Mutations in RMRP result in increased HIF1A levels during chondrogenesis. $12^{\text {th }}$ International Skeletal Dysplasia Society (ISDS) meeting, Istanbul, Turkey, July 29 - August 01, 2015

Steinbusch MMF, Caron MMJ, Eckmann F, Lausch E, van Rhijn LW, Welting TJM. Viperin; a novel chondrogenic regulator. Osteoarthritis Research Society International (OARSI) world congress on osteoarthritis, Seattle, WA, USA, April 30 - May 03, 2015 
Steinbusch MMF, Caron MMJ, Eckmann F, Lausch E, van Rhijn LW, Zabel B, Welting TJM. Viperin expression in chondrocytes is linked to expression of Col2a1. Orthopaedic Research Society (ORS) annual meeting, Las Vegas, NV, USA, March 28 - April 01, 2015

Steinbusch MMF, Caron MMJ, Eckmann F, Lausch E, van Rhijn LW, Zabel B, Welting TJM. Transcriptional regulation of the non-coding snoRNA RMRP during chondrogenic differentiation; a role for NFKB/p65? Osteoarthritis Research Society International (OARSI) world congress on osteoarthritis, Paris, France, April $24-27,2014$

Steinbusch MMF, Caron MMJ, Eckmann F, Lausch E, Zabel B, van Rhijn LW, Welting TJM. RNase MRP is involved in chondrogenic differentiation. RNA: the $18^{\text {th }}$ annual meeting of the RNA society, Davos, Switzerland, June $11-16,2013$

Eckmann F, Steinbusch MMF, Welting TJM, Wehrle A, Salfelder A, Keppler R, Janocha N, Lausch E, Zabel B. RMRP promoter mutation leads to increased promoter activity and Wntindependent expression of RMRP and p21. RNA: the $18^{\text {th }}$ annual meeting of the RNA society, Davos, Switzerland, June $11-16,2013$

Eckmann F, Steinbusch MMF, Welting TJM, Wehrle A, Salfelder A, Keppler R, Janocha N, Lausch E, Zabel B. Mutations in the RMRP promoter lead to increased promoter activity and changes in p21 expression. Third Heidelberg Forum for young scientists, Heidelberg, Germany, June $06-07,2013$ 
List of presentations 

List of honors

and achievements 


\section{List of honors and achievements}

\section{Honors and awards}

2014 - Best Oral Presentation Award Annual Conference Netherlands Society for Biomaterials and Tissue Engineering (NBTE)

2010-2011, 2009-2010 and 2007-2008 - Top 3\% certification - grant that gives the top 3\% of students enrolled in Maastricht University the opportunity to study free of charge by refunding their tuition fees

2010 - Prize for the best portfolio of the scientific lecture series "The biological basis of behavior", and portfolio to serve as template for future students

\section{Achievements}

2020 - Licensed Biosafety Officer (BVF 20-002) by the National Institute for Public Health and the Environment (RIVM / Bureau Genetisch Gemodificeerde Organismen)

2019 - Licensed Emergency first responder / Bedrijfs Hulpverlener in fire extinguishing, emergency evacuation, first-aid, resuscitation and use of the Automated External Defibrillator, Acaleph training institute. Trained as Intelex safety leader by Lonza Netherlands

2019 - Document Management System / Documentum key user and trouble shooter, Lonza

2018 - Quality Assurance technical writer Non-Conformance, Change Request, Corrective Actions Preventative Actions, Lonza Netherlands. Exam passed with 93/100\%

2013 - Radiation Protection level 5B. Licensed to work with open source radioactive materials, Maastricht University Radionuclide department

2011 - PhD course Statistics part II: regression analysis and SPSS. Exam passed with 9.4 / 10, honorary mention, Maastricht University

2011 - Safe Microbiological Techniques: certified for working with genetically modified organisms (GMOs) and pathogenic microorganisms (PMOs), Maastricht University 


\section{Specialized courses}

2019 and 2020 - Trained in Trackwise for raising deviations / non-conformances, corrective actions and preventative actions, and change requests, Lonza

2019 - Biosafety Officer course National Institute for Public Health and the Environment (RIVM / Bureau Genetisch Gemodificeerde Organismen) - trained to submit GGO permits and defining associated containment requirements

2019 - JMP Design of Experiments and statistics course

2019 - Certified to perform Purchase Requisitions in SAP, Lonza

2019 - Certified as Quality Assurance controlled copy holder, Lonza Netherlands

2018 - Thermofisher advanced ABI PCR course for Subject Matter Experts

\section{Teaching}

2017 - Supervisor Junior Master student Molecular Life Sciences Maastricht University: U3 snoRNA and rRNA processing and sequencing of rRNA heterogeneity

2016 - Lecturer Biomedical Sciences Master students, Maastricht University, Netherlands and Hasselt University, Belgium. Conception of exam questions. Subject: rRNA modifications and differential expression of snoRNAs in chondrogenic differentiation

2015 - Maastricht University Tutor Biomedical Sciences students: Epigenetics, miRNAs and biomarkers. Tutor Biomedical Sciences students: Organogenesis and embryonal development

2014 and 2015 - Practical instructor alkaline phosphatase determination Biomedical Sciences students, Maastricht University

2014 - Supervisor Junior Master student Molecular Life Sciences Maastricht University: fibrillarin; the box C/D snoRNA methyltransferase

\section{Extra-curricular activities}

2013 - 2017 - PhD panel member of the Maastricht University School for Public Health and Primary Care / Care and Public Health Research Institute (CAPHRI). Link between the 350 CAPHRI PhD candidates and the Educational Board 

Acknowledgements 


\section{Acknowledgements}

\section{A picture is worth a thousand words}

My time as a PhD candidate was a life phase filled with many highlights and new experiences. I have grown so much and I could not have done it without all of you. Please bear with me while I share some of my memories with you that in the end led to the completion of this PhD thesis. I thank you for being there every step of the way, guiding me, comforting me, and making me laugh.

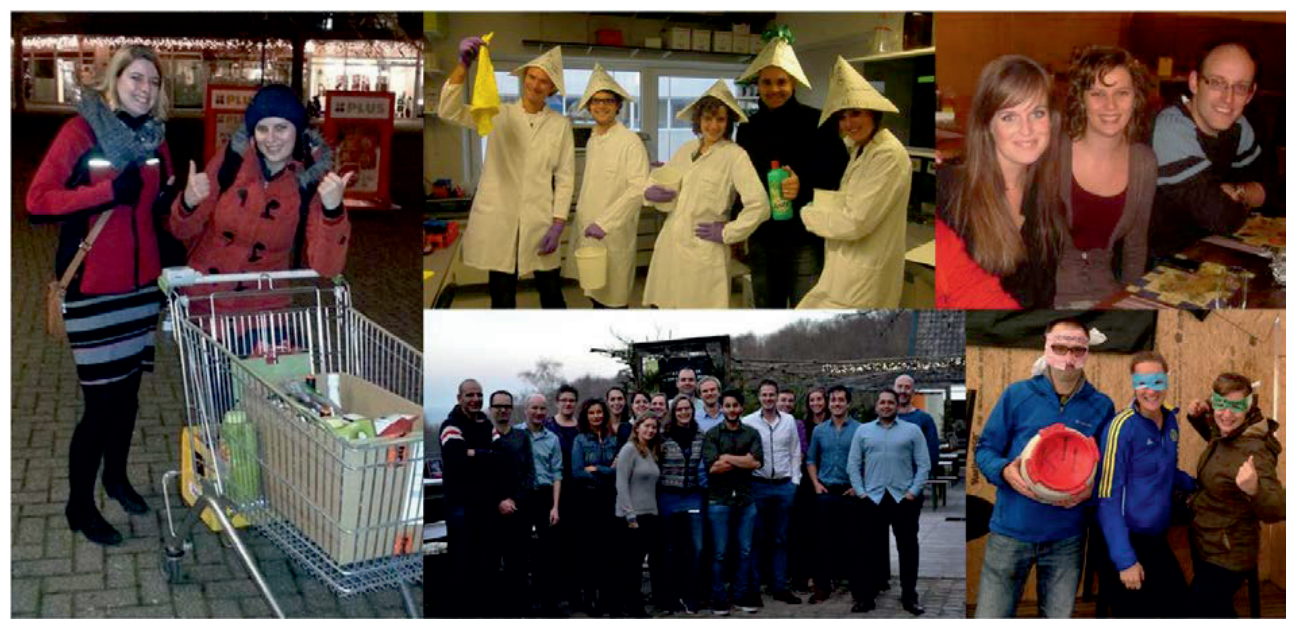

Lab cleaning, lab outings and lab friends. Ik wil het lab Orthopedie en de kliniek bedanken voor alle activiteiten die we samen hebben mogen beleven. Van shoppen voor de kerstborrel met Marjolein en glühwein in $50 \mathrm{~mL}$ buizen tot labschoonmaak met Alex, Maarten, Eva, Andy en Andy (het schoonmaakmiddel), van pubquiz en diner naast Eva en Jim tot een team vormen met Don en Marloes tijdens ons labuitje, ik heb overal van genoten. Bovendien greep ik altijd de kans aan om mijn moleculaire onderzoek uit te leggen aan Liesbeth, Pieter, Paul, Chris, Heleen, Adhiambo, Lodewijk en de overige lab- en stafleden tijdens de Orthopedic Research- en de pizzameetings. De vraag "maar wat is de klinische relevantie?" is eentje waar ik me van tevoren altijd goed op kon voorbereiden en ik werd dan ook nooit teleurgesteld omdat deze altijd gevraagd werd. 


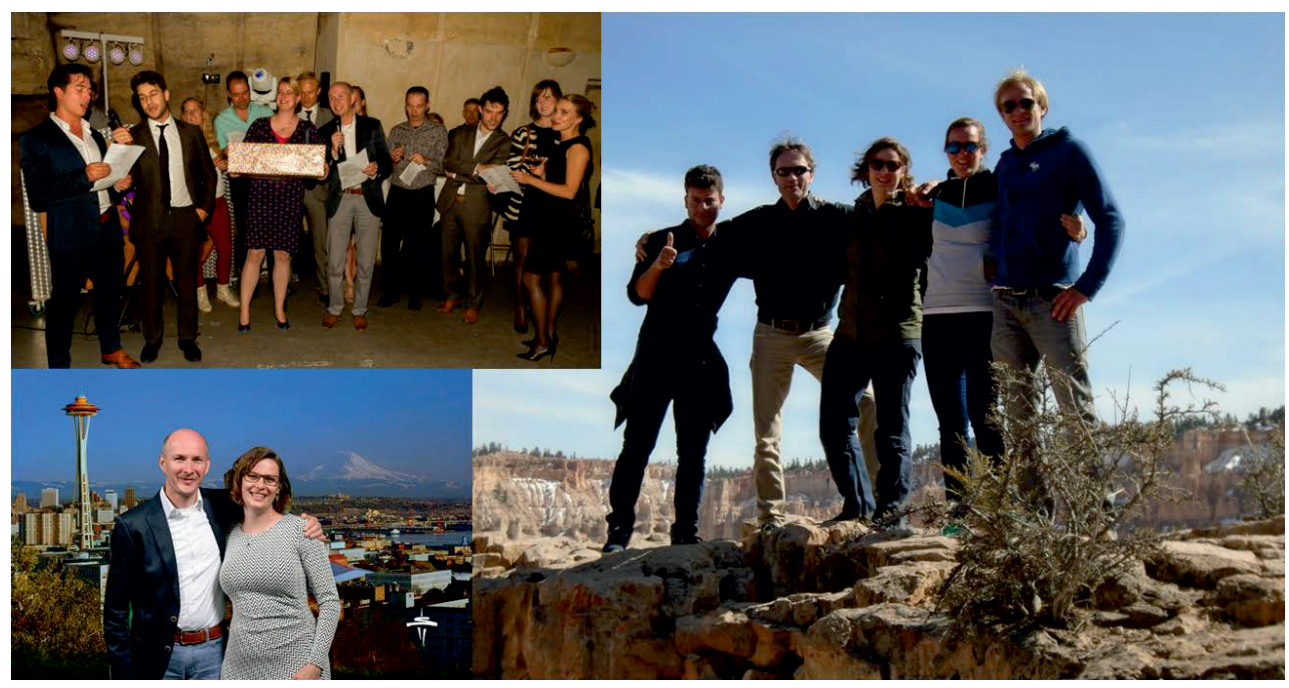

Lab activities and lab friends. Tussen alle experimenten door was er ook nog eens onze bruiloft, en het feit dat mijn labvrienden daarbij konden zijn maakte het een extra speciale dag. Ralph en Ufuk, jullie weergave van "Awimbawe, van LA naar viperin" tijdens onze bruiloft zal ik nooit meer vergeten. Twee andere hoogtepunten waren mijn bezoek aan de Space Needle (Speesj Needle) met Tim, en het Orthopedic Research Society congres in Las Vegas waarna we met Jeroen, Ufuk, Paul, Marloes en Alex een geweldige tocht door WestAmerika gemaakt hebben. Ik heb nog spierpijn van onze hikes door Bryce en Zion National park, maar het was $100 \%$ de moeite waard. Eddy, het is me gelukt. Bedankt voor onze veel te lange gesprekken op de gang. Florence, jij verdient een ereplek hier. Je was mijn eerste supervisor en mijn inspiratie om de PhD te starten. Goede keuze? Toch wel uiteindelijk. De orchidee van de bachelor heeft helaas vorig jaar het leven gegeven.

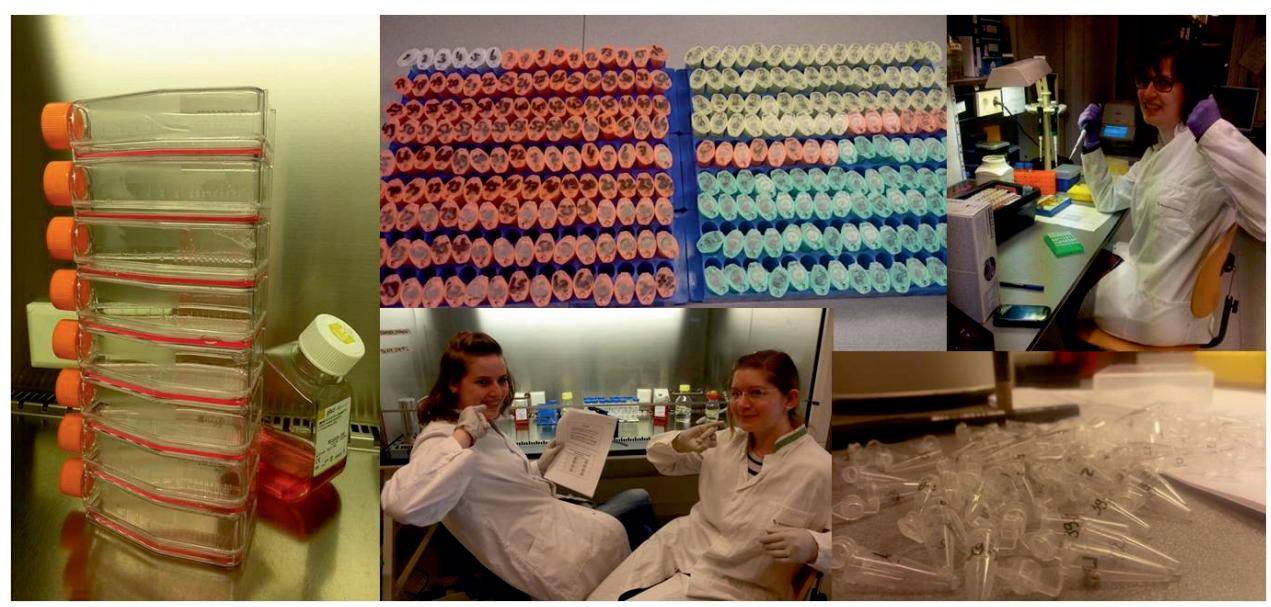


Experiments and Eppendorf tubes. Ik wil Tim bedanken voor alle kansen die hij me gegeven heeft. Een paar van de grootste voordelen van deze PhD waren de (internationale) collaboraties, de vele congressen, en de verschillende soorten experimenten die ik mocht uitvoeren. Van baanbrekend werk met LNAs (twee jaar optimalisatie, maar nooit geoptimaliseerd) tot fibroblastenkweek, samenwerking met Freiburg, Nijmegen en Liverpool en heel veel epjes. Ik wil ook Merel en Ger van harte bedanken voor de research meetings en de kans om Northern Blotting te leren. Ook heb ik veel van Wouter en Blanche geleerd tijdens de optimalisatie van de RMRP RNA In Situ Hybridisatie. Wat betreft PCR blijft het echter jammer dat het cDNA uit die kleine epjes altijd weer over gepipetteerd moest worden, vandaar dat ik vaak laat in de avond nog mijn experimenten aan het afronden was. Lodewijk, bedankt dat je mijn promotor wilde zijn. Ik kan me ons eerste gesprek herinneren toen jij op een kladblaadje uittekende hoe dat precies zat met die "RNAs", en tijdens ons tweede gesprek toverde je dit blaadje weer tevoorschijn. Ondanks dat mijn werk anders was, was jij altijd zeer enthousiast en ik denk dat je ook genoten hebt van onze wetenschappelijke discussies.

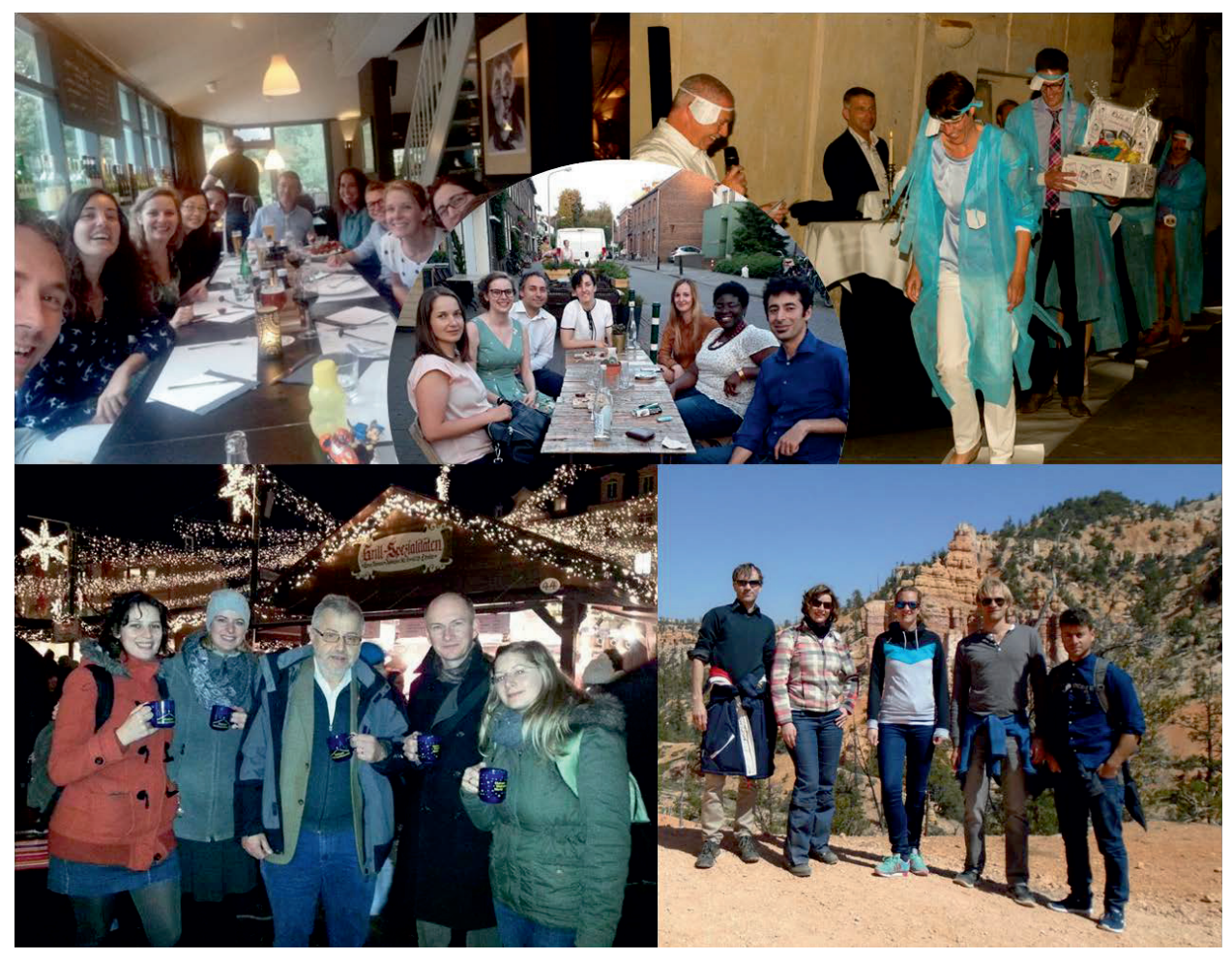


Adoptive departments. Tijdens mijn PhD werd ik al vroeg geadopteerd door mijn surrogaat labfamilie Klinische Genetica, waar Jeroen werkt. Marion, Frank, Wim, Laurence en Sabine, ik denk met liefde terug aan onze koffie corner breaks bij het Bakery café. Jullie zijn een geweldige, hechte groep, en iedereen die de kans heeft om bij jullie te werken mag van geluk spreken dat jullie zowel de "work-life balance" weten te onderhouden als kwalitatief en kwantitatief goed werk afleveren. Frank en Marion, onze skivakanties waren geweldig. In Val Cenis hebben we zelfs de acceptatie van mijn eerste paper gevierd. Marion, bedankt dat jij op 24 september achter mij zal staan als paranimf.

My second PhD family would be Lonza. I should have started a post-doc in Liège, instead Nasser tempted me (and actually hired me) to come and work at Lonza without any prior GMP knowledge. What a great decision I have made, haven't I? I know our group structure has changed, some of you left, others joined, but you all mean the world to me. A thank you to Benoit and lan for allowing me to take up some of my compensation hours to get this thesis out of the way. Thanks for the fun conversations Michael and Rein, I finished it! Moreover, the Freiburg family: Franzi, Prof. Dr. Zabel and Ekki. It was fascinating attending the "Fallbesprechungen", and I loved working at your lab where everything was allowed (if I were to compare it to Maastricht). You boosted my German skills and by the first week we did all the scientific discussions in German, although I will not attempt to write German here today as my grammar will surely be way off. I will say though: Ich wünsche Ihnen alles Gute.

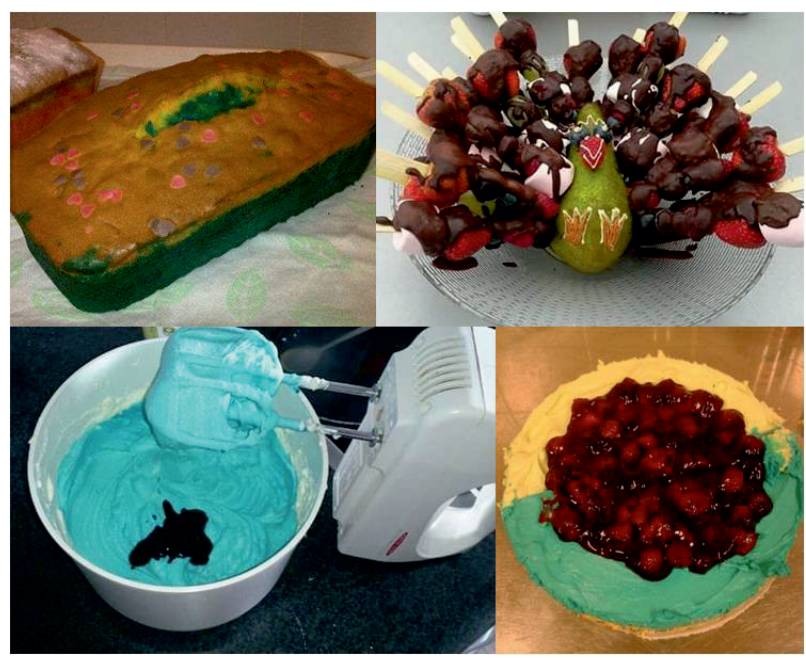

Cake of the week. De cake van de week was een creatieve en lekkere onderbreking bedacht door Marjolein. Ik baalde iedere keer weer als ik op de lijst stond (en vaak bakte Jeroen, psst), maar ik kijk met genoegen terug op mijn kleurige E-nummer creaties. Ik hoop jullie ook! Minder goed voor de lijn, maar een geweldige pauze! 


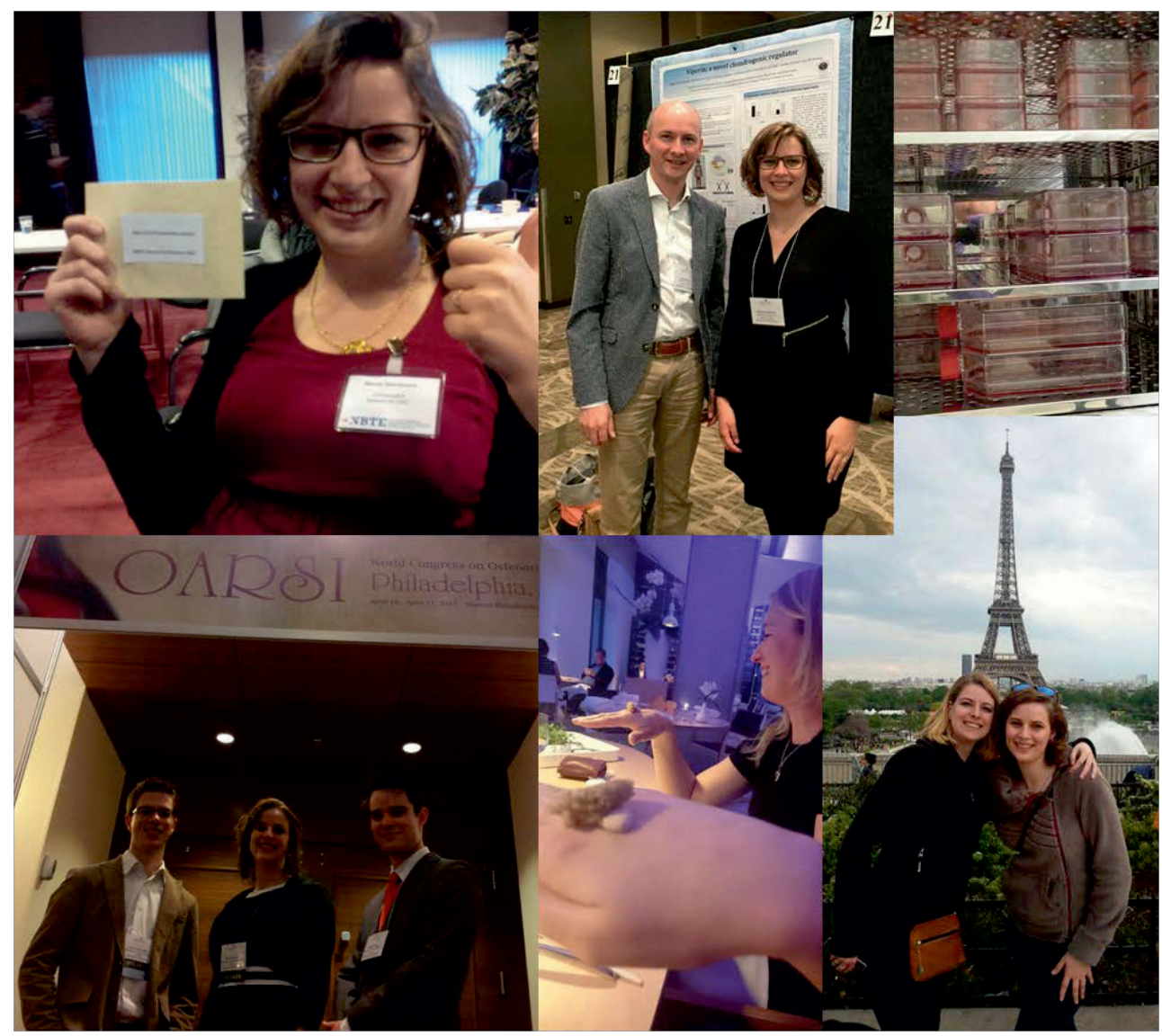

Conferences. Daar heb je ze: congressen! Door heel Europa en Amerika heb ik kunnen reizen. Guus en Maarten, jullie waren erbij tijdens mijn eerste congres en eerste reis naar Amerika voor de OARSI in Philadelphia, waar ik ook nog eens meteen een "oral presentation" had. De OARSI heb ik ook gezien met Tim in Seattle en met Marjolein in Parijs. Dichterbij huis was het NBTE congres in Lunteren een echte highlight, waar ik zelfs in 2014 de "Best Oral Presentation Prize" gewonnen heb.

Mandy Peffers, and all the other Liverpool colleagues, I feel truly blessed to have been given the opportunity to work at Leahurst for three months. I even got to work with the horses. Mandy, with great pleasure I think back to our OARSI dinner in Amsterdam. I, for one, cannot forget eating that food from the top of our hands. Thank you for inviting me into your home. I have never met a more welcoming, smart, beautiful, strong Professor who can handle it all! You are truly an inspiration and I am humbled I had the chance to publish a paper with you. 


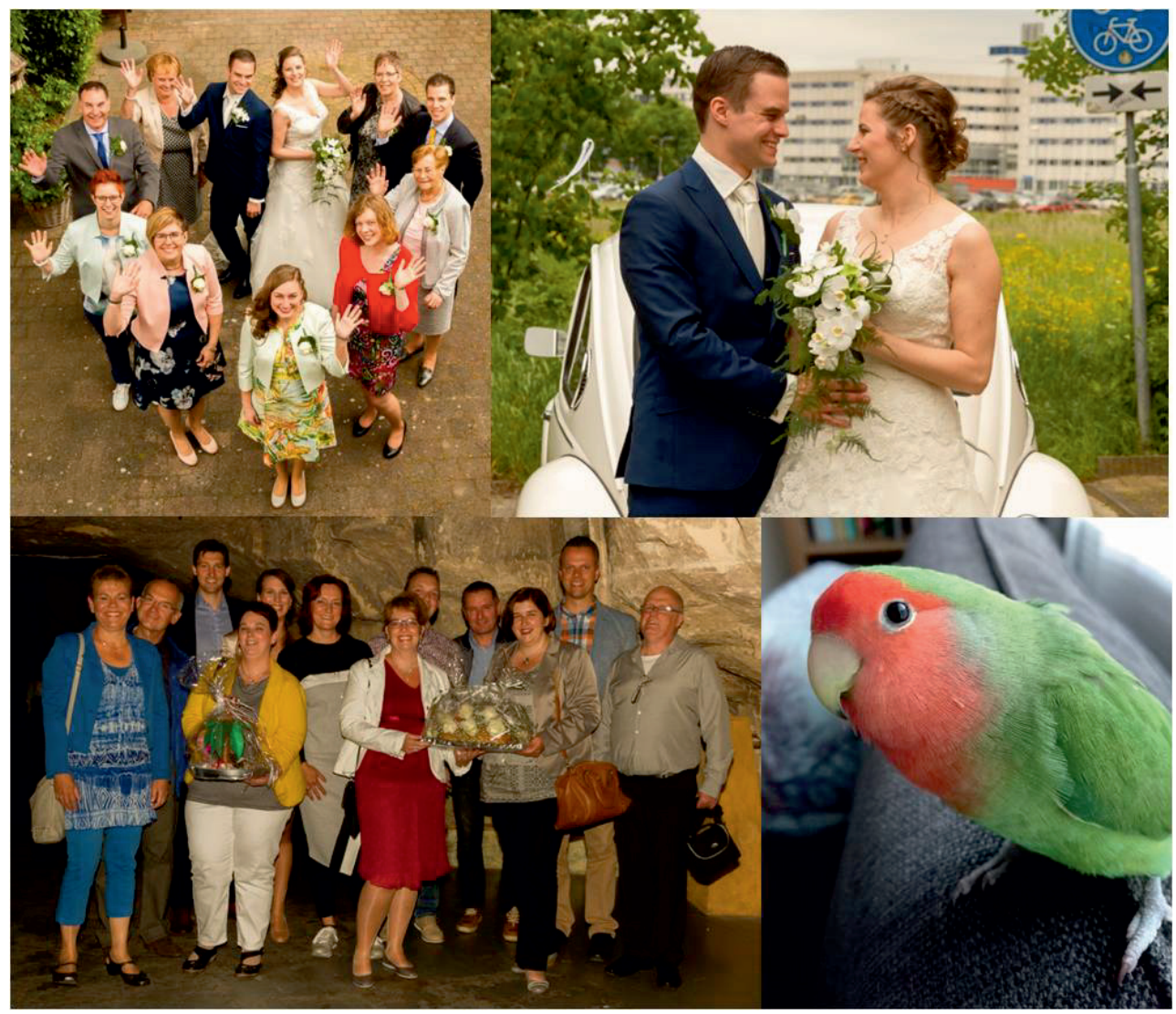

Family, friends and the best husband I could wish for. Zonder familie en vrienden om het werk te relativeren had ik het niet gekund. Ik wil iedereen op de foto bedanken (ook Mireille, Frank en Sierk die helaas niet op deze foto's staan). Familie Meekels, bedankt voor de barbecues bij haardvuur. Audrey en Dorien, dank jullie wel dat jullie mij tijdens deze periode bijgestaan hebben als vrienden en getuigen. Dorien, jij zal tevens achter mij staan op 24 september als een van mijn paranimfen. Rick, je begrijpt misschien niks van mijn onderzoek, maar je bent wel mijn broer die me weg heeft gegeven aan Jeroen tijdens de bruiloft. En niet alles hoeft uiteraard over werk te gaan. Jokola was mijn afleiding en de problemen over kledingkeuzestress leken triviaal ten opzichte van andere beslissingen die ik soms moest nemen. Daar kon ik altijd zeer om lachen. Ik zie het bestuur als mijn vrienden. En Nance, jij verdient een ereplaatsje in dit dankwoord. Deze voorzitter kan niet zonder de beste secretaresse en daarnaast ben je gewoon een fantastisch en begripvol mens waar ik graag mee app en die altijd bereid is me te helpen. Ook Noet mag niet ontbreken, ik ben blij dat jij me door het grootste deel van de PhD hebt kunnen bijstaan met je knuffels en kusjes. 
Lieve Jeroen, zonder jou had ik de eindstreep niet gered. Terwijl ik dit dankwoord schrijf probeer jij mijn Word bestanden aan te passen voor de drukker. Nog geen week geleden was je doordeweeks tot half 3 's nachts bezig om last minute "copyright clearance" te regelen voor alle figuren in hoofdstuk 1. Je hebt zelfs de originele artiest van de cover art gevonden. And so, I would like to thank professional visual artist Miss René Teresa Campbell for letting me use her amazing art work "The fabric of life" as my cover art, and I wish her well in finishing her own PhD thesis in the upcoming months.

Jeroen, je bent er altijd voor mij als ik je nodig heb. Ik hou ervan dat we samen de Master of Science gedaan hebben, en dat ik mijn werk met je kan bespreken, dat we het regelmatig in de auto over DNA, genen, trophectoderm en allelic dropout hebben, dat je begrijpt wat ik doormaak, dat je mijn rust en tegenhanger bent, dat je me zegt dat ik pauzes moet nemen en dat we iets leuks gaan doen of juist dat ik het moet afronden, en dat je begrijpt dat ik soms keuzes heb moeten maken. Hopelijk kun je net als ik ook terugkijken op de afgelopen jaren en genieten van alle mooie momenten die we hebben meegemaakt, zoals ons trouwfeest en de reis door Amerika na de ORS conferentie met het lab. Gezien mijn toewijding aan mijn werk is het misschien niet geheel raar dat de achtergrond van onze trouwfoto Maastricht University en het Academisch Ziekenhuis Maastricht was; lekker dicht bij het werk wonen was wel zo prettig voor het avond- en weekendwerk. Ik ben ook opgelucht dat dit hoofdstuk nu afgesloten is en we ons kunnen richten op een nieuw tijdperk. Ik kan niet wachten om onze kleine binnenkort te ontmoeten, en ik hoop dat 'ie zich gedraagt tijdens de verdediging. 